\title{
Macrocyclic $\beta$-Sheet Peptides that Mimic Protein Quaternary Structure through Intermolecular $\beta$-Sheet Interactions
}

\author{
Omid Khakshoor ${ }^{\dagger}$ Borries Demeler ${ }^{\ddagger}$ and James $S$. Nowick ${ }^{* \dagger}$ \\ Department of Chemistry, University of California, Irvine, Irvine, CA 92697-2025 \\ Department of Biochemistry, University of Texas Health Science Center, San Antonio, TX 78229 \\ E-mail: jsnowick@uci.edu
}

\section{Contents}

$\begin{array}{lr}\text { Synthetic procedures } & 6\end{array}$

$\begin{array}{ll}\text { Analytical RP-HPLC of peptide 3a } & 17\end{array}$

$\begin{array}{lr}\text { LRMS (ESI) of peptide 3a } & 18\end{array}$

Analytical RP-HPLC of peptide 3b 19

$\begin{array}{lr}\text { LRMS (ESI) of peptide 3b } & 20\end{array}$

$\begin{array}{ll}\text { Analytical RP-HPLC of peptide 3c } & 21\end{array}$

LRMS (ESI) of peptide 3c $\quad 22$

Analytical RP-HPLC of peptide 3d 23

LRMS (ESI) of peptide 3d $\quad 24$

Analytical RP-HPLC of peptide 3e 25

LRMS (ESI) of peptide 3e 26

$\begin{array}{ll}\text { Analytical RP-HPLC of peptide } \mathbf{3 f} & 27\end{array}$

$\begin{array}{lr}\text { LRMS (ESI) of peptide } \mathbf{3 f} & 28\end{array}$

$\begin{array}{ll}\text { Analytical RP-HPLC of peptide 3g } & 29\end{array}$

LRMS (ESI) of peptide 3g $\quad 30$

Analytical RP-HPLC of peptide $\mathbf{3 h} \quad 31$

LRMS (ESI) of peptide 3h $\quad 32$

Analytical RP-HPLC of peptide 3i 33

$\dagger$ University of California, Irvine, CA

†University of Texas Health Science Center, San Antonio, TX 
LRMS (ESI) of peptide 3i $\quad 34$

Analytical RP-HPLC of peptide 3j

LRMS (ESI) of peptide 3j

Analytical RP-HPLC of peptide 3k 37

LRMS (ESI) of peptide 3k 38

Analytical RP-HPLC of peptide 31 39

LRMS (ESI) of peptide 3l $\quad 40$

Analytical RP-HPLC of peptide 3m

LRMS (ESI) of peptide 3m 42

Analytical RP-HPLC of peptide 3n 43

LRMS (ESI) of peptide 3n $\quad 44$

Analytical RP-HPLC of peptide 6

LRMS (ESI) of peptide $6 \quad 46$

$\begin{array}{ll}{ }^{1} \mathrm{H} \text { NMR Characterization of peptide 3a } & 47\end{array}$

${ }^{1} \mathrm{H}$ NMR spectra of the oligomer of peptide $3 \mathbf{a}$ in $\mathrm{D}_{2} \mathrm{O}$ (at $8.5 \mathrm{mM}$ concentration) 48

$800 \mathrm{MHz} 1 \mathrm{D}{ }^{1} \mathrm{H}$ NMR of the oligomer of peptide 3a

$800 \mathrm{MHz}$ TOCSY spectrum of the oligomer of peptide 3a

$800 \mathrm{MHz}$ NOESY spectrum of the oligomer of peptide 3a

$500 \mathrm{MHz}$ ROESY spectrum of the oligomer of peptide 3a

${ }^{1} \mathrm{H}$ NMR spectra of the oligomer of peptide $3 \mathbf{a}$ in $\mathrm{H}_{2} \mathrm{O}-\mathrm{D}_{2} \mathrm{O}(9: 1)$ (at $8.5 \mathrm{mM}$ concentration) 58 $800 \mathrm{MHz} 1 \mathrm{D}{ }^{1} \mathrm{H}$ NMR of the oligomer of peptide 3a

$800 \mathrm{MHz}$ TOCSY spectrum of the oligomer of peptide 3a

$800 \mathrm{MHz}$ NOESY spectrum of the oligomer of peptide 3a 61

$800 \mathrm{MHz}$ EXSY spectrum of the oligomer of peptide 3a 67

Key NOEs in the oligomer of peptide 3a $\quad 68$

${ }^{1} \mathrm{H}$ NMR spectra of the monomer of peptide 3a in $\mathrm{D}_{2} \mathrm{O}$ (at $1.0 \mathrm{mM}$ concentration) 70 $800 \mathrm{MHz} 1 \mathrm{D}{ }^{1} \mathrm{H}$ NMR of the monomer of peptide 3a $\quad 70$

$800 \mathrm{MHz}$ TOCSY spectrum of the monomer of peptide 3a $\quad 72$

$800 \mathrm{MHz}$ NOESY spectrum of the monomer of peptide 3a $\quad 74$

${ }^{1} \mathrm{H}$ NMR spectra of the monomer of peptide 3a in $\mathrm{H}_{2} \mathrm{O}-\mathrm{D}_{2} \mathrm{O}(9: 1)$ (at $0.6 \mathrm{mM}$ concentration) 79 $800 \mathrm{MHz} 1 \mathrm{D}{ }^{1} \mathrm{H}$ NMR of the monomer of peptide 3a 
$800 \mathrm{MHz}$ TOCSY spectrum of the monomer of peptide 3a

$800 \mathrm{MHz}$ NOESY spectrum of the monomer of peptide $\mathbf{3 a}$

Key NOEs in the monomer of peptide 3a $\quad 84$

${ }^{1} \mathrm{H}$ NMR concentration studies of peptide 3a in $\mathrm{D}_{2} \mathrm{O} \quad 85$

Pulsed Field Gradient (PFG) NMR diffusion coefficient measurements of peptide 3a $\quad 87$

Analytical ultracentrifugation studies of peptide 3a 91

${ }^{1} \mathrm{H}$ NMR studies of control peptide $6 \quad 94$

$500 \mathrm{MHz}{ }^{1} \mathrm{H}$ NMR spectra of linear peptide 6 in $\mathrm{D}_{2} \mathrm{O}$

1D ${ }^{1} \mathrm{H}$ NMR of control peptide 6

TOCSY spectrum of control peptide $6 \quad 96$

ROESY spectrum of control peptide $6 \quad 97$

${ }^{1} \mathrm{H}$ NMR studies of peptides 3a-3n $\quad 98$

$500 \mathrm{MHz}{ }^{1} \mathrm{H}$ NMR spectra of peptide $\mathbf{3 a}$ in $\mathrm{D}_{2} \mathrm{O} \quad 99$

1D ${ }^{1} \mathrm{H}$ NMR of the monomer of peptide 3a 99

$500 \mathrm{MHz}{ }^{1} \mathrm{H}$ NMR spectra of peptide $\mathbf{3 b}$ in $\mathrm{D}_{2} \mathrm{O} \quad 100$

1D ${ }^{1} \mathrm{H}$ NMR of the monomer of peptide $\mathbf{3 b} \quad 100$

TOCSY spectrum of the monomer of peptide $\mathbf{3 b} \quad 101$

ROESY spectrum of the monomer of peptide $\mathbf{3 b} \quad 102$

Overlay of $1 \mathrm{D}{ }^{1} \mathrm{H}$ NMR spectra of dilute and concentrated solutions of peptide $\mathbf{3 b} \quad 103$

$500 \mathrm{MHz}{ }^{1} \mathrm{H}$ NMR spectra of peptide $\mathbf{3 c}$ in $\mathrm{D}_{2} \mathrm{O} \quad 104$

1D ${ }^{1} \mathrm{H}$ NMR of the monomer of peptide 3c 104

TOCSY spectrum of the monomer of peptide 3c 105

ROESY spectrum of the monomer of peptide 3c 106

Overlay of 1D ${ }^{1} \mathrm{H}$ NMR spectra of dilute and concentrated solutions of peptide 3c 107 $500 \mathrm{MHz}{ }^{1} \mathrm{H}$ NMR spectra of peptide 3d in $\mathrm{D}_{2} \mathrm{O} \quad 108$

1D ${ }^{1} \mathrm{H}$ NMR of the monomer of peptide 3d 108

TOCSY spectrum of the monomer of peptide 3d 109

ROESY spectrum of the monomer of peptide 3d 110

Overlay of 1D ${ }^{1} \mathrm{H}$ NMR spectra of dilute and concentrated solutions of peptide 3d 111

$500 \mathrm{MHz}{ }^{1} \mathrm{H}$ NMR spectra of peptide $\mathbf{3 e}$ in $\mathrm{D}_{2} \mathrm{O}$

1D ${ }^{1} \mathrm{H}$ NMR of the monomer of peptide $3 \mathbf{e}$ 
TOCSY spectrum of the monomer of peptide $\mathbf{3 e}$

ROESY spectrum of the monomer of peptide $3 \mathbf{e}$

Overlay of $1 \mathrm{D}^{1} \mathrm{H}$ NMR spectra of dilute and concentrated solutions of peptide $3 \mathbf{e} 115$ $500 \mathrm{MHz}{ }^{1} \mathrm{H}$ NMR spectra of peptide $\mathbf{3 f}$ in $\mathrm{D}_{2} \mathrm{O}$

1D ${ }^{1} \mathrm{H}$ NMR of the monomer of peptide $3 \mathbf{f}$

TOCSY spectrum of the monomer of peptide $\mathbf{3 f} \quad 117$

ROESY spectrum of the monomer of peptide $\mathbf{3 f} \quad 118$

Overlay of $1 \mathrm{D}{ }^{1} \mathrm{H}$ NMR spectra of dilute and concentrated solutions of peptide $3 \mathbf{f} \quad 119$

$500 \mathrm{MHz}{ }^{1} \mathrm{H}$ NMR spectra of peptide $\mathbf{3 g}$ in $\mathrm{D}_{2} \mathrm{O} \quad 120$

1D ${ }^{1} \mathrm{H}$ NMR of the monomer of peptide $\mathbf{3 g} \quad 120$

TOCSY spectrum of the monomer of peptide $\mathbf{3 g} \quad 121$

ROESY spectrum of the monomer of peptide $\mathbf{3 g} \quad 122$

Overlay of $1 \mathrm{D}^{1} \mathrm{H}$ NMR spectra of dilute and concentrated solutions of peptide $\mathbf{3 g} 123$

$500 \mathrm{MHz}{ }^{1} \mathrm{H}$ NMR spectra of peptide $\mathbf{3 h}$ in $\mathrm{D}_{2} \mathrm{O}$

1D ${ }^{1} \mathrm{H}$ NMR of the monomer of peptide $\mathbf{3 h} \quad 124$

TOCSY spectrum of the monomer of peptide $\mathbf{3 h} \quad 125$

ROESY spectrum of the monomer of peptide $\mathbf{3 h} \quad 126$

Overlay of 1D ${ }^{1} \mathrm{H}$ NMR spectra of dilute and concentrated solutions of peptide $3 \mathbf{h} \quad 127$

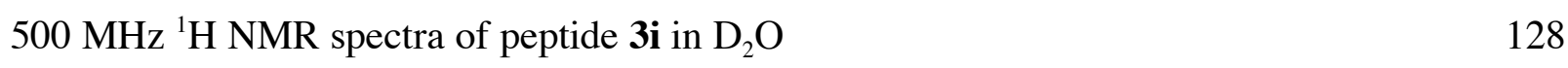

1D ${ }^{1} \mathrm{H}$ NMR of peptide 3i 128

TOCSY spectrum of peptide 3i 129

ROESY spectrum of peptide 3i $\quad 130$

Overlay of $1 \mathrm{D}^{1} \mathrm{H}$ NMR spectra of dilute and concentrated solutions of peptide $3 \mathbf{i} \quad 131$

$500 \mathrm{MHz}{ }^{1} \mathrm{H}$ NMR spectra of peptide $\mathbf{3 j}$ in $\mathrm{D}_{2} \mathrm{O}$

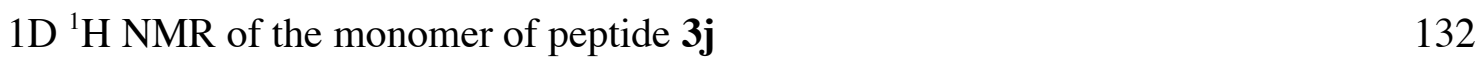

TOCSY spectrum of the monomer of peptide $\mathbf{3 j}$

ROESY spectrum of the monomer of peptide $\mathbf{3 j}$

Overlay of 1D ${ }^{1} \mathrm{H}$ NMR spectra of dilute and concentrated solutions of peptide $\mathbf{3 j} \quad 135$ $500 \mathrm{MHz}{ }^{1} \mathrm{H}$ NMR spectra of peptide $3 \mathbf{k}$ in $\mathrm{D}_{2} \mathrm{O}$

1D ${ }^{1} \mathrm{H}$ NMR of the monomer of peptide 3k 136

TOCSY spectrum of the monomer of peptide $\mathbf{3 k}$ 
ROESY spectrum of the monomer of peptide $\mathbf{3 k}$

Overlay of $1 \mathrm{D}{ }^{1} \mathrm{H}$ NMR spectra of dilute and concentrated solutions of peptide $3 \mathbf{k} 139$

$500 \mathrm{MHz}{ }^{1} \mathrm{H}$ NMR spectra of peptide $3 \mathrm{I}$ in $\mathrm{D}_{2} \mathrm{O}$

1D ${ }^{1} \mathrm{H}$ NMR of peptide 3l $\quad 140$

TOCSY spectrum of peptide 3I 141

ROESY spectrum of peptide 3I 142

Overlay of $1 \mathrm{D}{ }^{1} \mathrm{H}$ NMR spectra of dilute and concentrated solutions of peptide $3 \mathrm{I} \quad 143$

$500 \mathrm{MHz}{ }^{1} \mathrm{H}$ NMR spectra of peptide 3m in $\mathrm{D}_{2} \mathrm{O}$

1D ${ }^{1} \mathrm{H}$ NMR of peptide $3 \mathbf{m}$

TOCSY spectrum of peptide $\mathbf{3 m} \quad 145$

ROESY spectrum of peptide 3m 146

Overlay of $1 \mathrm{D}{ }^{1} \mathrm{H}$ NMR spectra of dilute and concentrated solutions of peptide $\mathbf{3 m} 147$

$500 \mathrm{MHz}{ }^{1} \mathrm{H}$ NMR spectra of peptide $3 \mathbf{n}$ in $\mathrm{D}_{2} \mathrm{O}$

1D ${ }^{1} \mathrm{H}$ NMR of peptide 3n 148

TOCSY spectrum of peptide $\mathbf{3 n} \quad 149$

$\begin{array}{lr}\text { ROESY spectrum of peptide 3n } & 150\end{array}$

Overlay of $1 \mathrm{D}{ }^{1} \mathrm{H}$ NMR spectra of dilute and concentrated solutions of peptide 3n 151

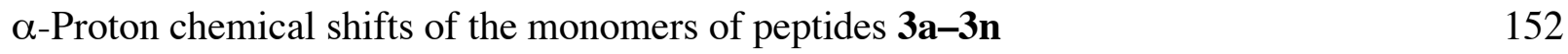

$\Delta \delta^{\delta}$ Orn values for the monomers of peptides 3a-3n 153 


\section{Synthetic procedures}

General. Toluene and $\mathrm{CH}_{2} \mathrm{Cl}_{2}$ were dried by filtration through alumina according to the procedure of Grubbs. ${ }^{1}$ TGT alcohol resin was purchased from Novabiochem. Lysozyme (from chicken egg white) and ubiquitin were obtained from Sigma-Aldrich. Gramicidin S was purchased from Cambridge Isotope Laboratories, Inc. in $\mathrm{CD}_{3} \mathrm{SOCD}_{3}$ solution and purified by RP-HPLC. ${ }^{2}$ Fritted Bio-Rad Poly-prep chromatography columns $(0.8 \times 4 \mathrm{~cm})$ were used as plastic reaction vessels for resin loading and cleavage of peptide from resin. Automated continuous solid-phase peptide synthesis was carried out on a PerSeptive Biosystems Pioneer ${ }^{\mathrm{TM}}$ Peptide Synthesis system. Analytical reverse phase HPLC (RP-HPLC) was performed on a Beckman (Agilent Zorbax $80 \mathrm{SB} \mathrm{C}_{18}$ column, 50 x $4.6 \mathrm{~mm}$; solvent A: $\mathrm{H}_{2} \mathrm{O} / 0.1 \%$ TFA, solvent B: $\mathrm{CH}_{3} \mathrm{CN} / 0.1 \%$ TFA), and preparative RP-HPLC was carried out on a Rainin machine (Varian Dynemax Microsorb 100-5 $\mathrm{C}_{18}$ column, 250 x $21.4 \mathrm{~mm}$; solvent A: $\mathrm{H}_{2} \mathrm{O} / 0.1 \%$ TFA, solvent B: $\mathrm{CH}_{3} \mathrm{CN} / 0.1 \%$ TFA). ${ }^{1} \mathrm{H}$ NMR Spectra were acquired either on a Bruker DRX500 (500 MHz) spectrometer, on a Bruker Cryoprobe (500 MHz) spectrometer, or on a Varian INOVA800 (800 $\mathrm{MHz}$ ) spectrometer. NMR data were processed by using Bruker XWINNMR software (except for PFG NMR diffusion measurements, for which VNMR software was used for processing), and were reported in ppm using DSA as internal standard. ${ }^{3}$

\section{PS-PEG-trityl chloride resin.}

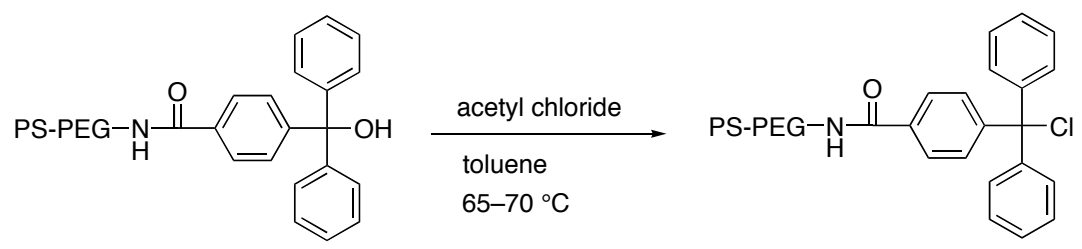

PS-PEG-trityl alcohol resin (TGT alcohol resin)

PS-PEG-trityl chloride resin

A flame-dried 100-mL one-necked round-bottomed flask equipped with a condenser connected to a $\mathrm{N}_{2}$ inlet adapter was charged with PS-PEG-trityl alcohol resin (Novabiochem

1 Pangborn, A. B.; Giardello, M. A.; Grubbs, R. H.; Rosen, R. K.; Timmers, F. J. Organometallics 1996, 15, 15181520.

2 For a synthesis procedure: Grotenbreg, G. M.; Kronemeijer, M.; Timmer, M. S.; El Oualid, F.; van Well, R. M.; Verdoes, M.; Spalburg, E.; van Hooft, P. A.; de Neeling, A. J.; Noort, D.; van Boom, J. H.; van der Marel, G. A.; Overkleeft, H.S. ; Overhand, M. J. Org. Chem. 2004, 69, 7851-7859.

3 Nowick, J. S.; Khakshoor, O.; Hashemzadeh, M.; Brower, J. O. Org. Lett. 2003, 5, 3511-3513. 
NovaSyn TGT alcohol resin, $0.36 \mathrm{~g}, 0.27 \mathrm{mmol} / \mathrm{g}$ loading, $0.097 \mathrm{mmol}$ ), and the resin was covered with dry toluene $(20 \mathrm{~mL})$. Freshly distilled acetyl chloride $(1.0 \mathrm{~mL}, 1.1 \mathrm{~g}, 14 \mathrm{mmol})$ was added to the flask, and the mixture was heated at reflux with a $65-70{ }^{\circ} \mathrm{C}$ oil bath under $\mathrm{N}_{2}$ for 3 h. The slurry was then transferred to a dry fritted plastic reaction vessel, and the solution was drained to give the PS-PEG-trityl chloride resin. The resin was then washed with dry toluene $(3 \mathrm{x}$ $5 \mathrm{~mL})$ and dry $\mathrm{CH}_{2} \mathrm{Cl}_{2}(3 \times 5 \mathrm{~mL})$ and used immediately in the next step.

\section{PS-PEG-trityl-Orn(Fmoc)-Boc resin.}

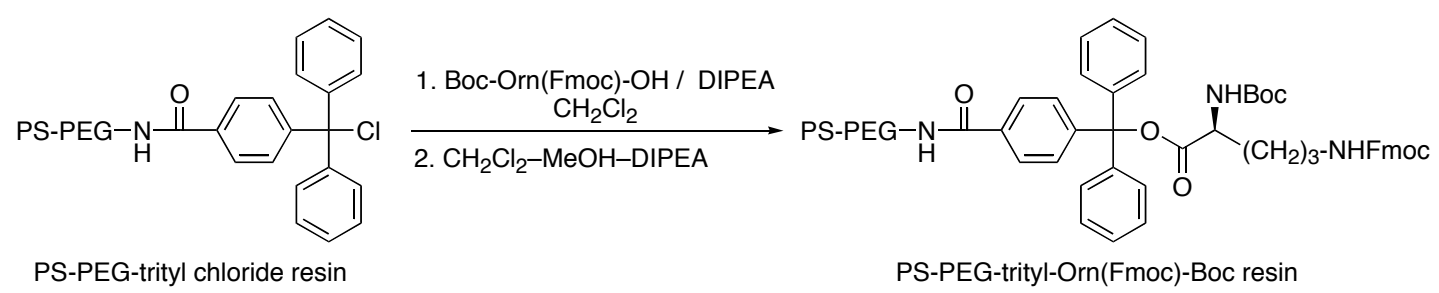

To the PS-PEG-trityl chloride resin $(0.097 \mathrm{mmol})$ in the fritted plastic reaction vessel, was added a solution of Boc-Orn(Fmoc)-OH (0.12 g, $0.26 \mathrm{mmol}, 2.7$ equiv) and DIPEA (0.10 $\mathrm{mL}, 0.58 \mathrm{mmol}, 6.0$ equiv) in $\mathrm{CH}_{2} \mathrm{Cl}_{2}(5 \mathrm{~mL})$. The reaction vessel was shaken for $2 \mathrm{~h}$ at room temperature, and the solution was drained. The resin was again shaken with a solution of BocOrn(Fmoc)-OH (0.060 g, $0.13 \mathrm{mmol}, 1.4$ equiv) and DIPEA (0.050 mL, $0.29 \mathrm{mmol}, 3.0$ equiv) in $\mathrm{CH}_{2} \mathrm{Cl}_{2}(3 \mathrm{~mL})$ for $1 \mathrm{~h}$. The resin was then washed with $\mathrm{CH}_{2} \mathrm{Cl}_{2}(2 \times 5 \mathrm{~mL})$ and was then shaken with $\mathrm{CH}_{2} \mathrm{Cl}_{2}-\mathrm{MeOH}-\mathrm{DIPEA}(17: 2: 1(\mathrm{v} / \mathrm{v}), 2 \times 7 \mathrm{~mL}, 1 \mathrm{~h}$ each time). The solution was drained, and the resin was washed with $\mathrm{CH}_{2} \mathrm{Cl}_{2}(3 \times 5 \mathrm{~mL}), \mathrm{DMF}(2 \times 5 \mathrm{~mL}), \mathrm{CH}_{2} \mathrm{Cl}_{2}(3 \times 5$ $\mathrm{mL})$, and hexanes $(2 \times 5 \mathrm{~mL})$. The resin was dried under $\mathrm{N}_{2}$ for $12 \mathrm{~h}$, and the loading of the resin was determined using UV spectroscopy: ${ }^{4}$ A small portion of the resin (ca. $5 \mathrm{mg}$ ) was Fmocdeprotected with $20 \%$ piperidine in DMF (3 mL, $3 \mathrm{~min}$ ), the optical density of the supernatant was measured at $290 \mathrm{~nm}$, and the loading was determined to be $0.145 \mathrm{mmol} / \mathrm{g}(60 \%){ }^{5}$

4 Novabiochem catalog 2002/3, Synthesis notes section.

5 A $50-90 \%$ loading of the resin is usually obtained with this procedure. 


\section{Protected linear peptides 4:}

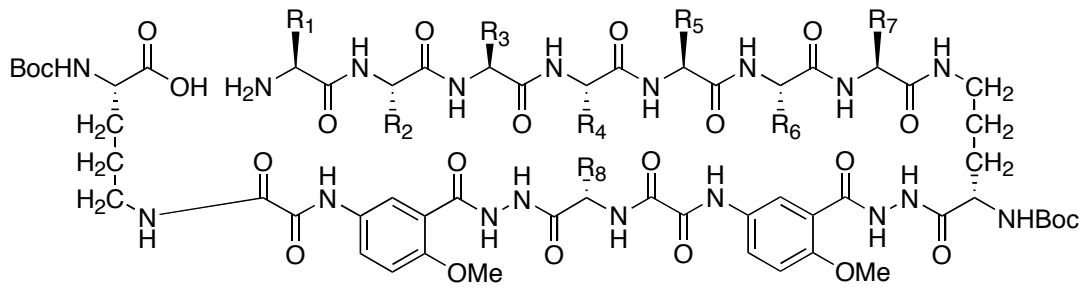

\begin{tabular}{|c|c|c|c|c|c|c|c|c|}
\hline Peptide & $\mathbf{R}_{1}$ & $\mathbf{R}_{2}$ & $\mathbf{R}_{3}$ & $\mathbf{R}_{\mathbf{4}}$ & $\mathbf{R}_{5}$ & $\mathbf{R}_{6}$ & $\mathbf{R}_{7}$ & $\mathbf{R}_{8}$ \\
\hline $4 a$ & $\operatorname{Thr}(t \mathrm{Bu})$ & $\operatorname{Ser}(t \mathrm{Bu})$ & Phe & $\operatorname{Thr}(t \mathrm{Bu})$ & $\operatorname{Tyr}(t \mathrm{Bu})$ & $\operatorname{Thr}(t \mathrm{Bu})$ & $\operatorname{Ser}(t \mathrm{Bu})$ & Lys(Boc) \\
\hline $4 b$ & $\operatorname{Thr}(t \mathrm{Bu})$ & $\operatorname{Ser}(t \mathrm{Bu})$ & Phe & $\operatorname{Glu}(t \mathrm{Bu})$ & $\operatorname{Tyr}(t \mathrm{Bu})$ & $\operatorname{Thr}(t \mathrm{Bu})$ & $\operatorname{Ser}(t \mathrm{Bu})$ & Lys(Boc) \\
\hline $4 c$ & $\operatorname{Thr}(t \mathrm{Bu})$ & $\operatorname{Ser}(t \mathrm{Bu})$ & Phe & Leu & $\operatorname{Tyr}(t \mathrm{Bu})$ & $\operatorname{Thr}(t \mathrm{Bu})$ & $\operatorname{Ser}(t \mathrm{Bu})$ & Lys(Boc) \\
\hline $4 d$ & $\operatorname{Thr}(t \mathrm{Bu})$ & $\operatorname{Ser}(t \mathrm{Bu})$ & Phe & $\operatorname{Tyr}(t \mathrm{Bu})$ & $\operatorname{Tyr}(t \mathrm{Bu})$ & $\operatorname{Thr}(t \mathrm{Bu})$ & $\operatorname{Ser}(t \mathrm{Bu})$ & Lys(Boc) \\
\hline $4 e$ & $\operatorname{Thr}(t \mathrm{Bu})$ & Leu & Phe & $\operatorname{Thr}(t \mathrm{Bu})$ & $\operatorname{Tyr}(t \mathrm{Bu})$ & Val & $\operatorname{Ser}(t \mathrm{Bu})$ & Lys(Boc) \\
\hline $\mathbf{4 f}$ & $\operatorname{Thr}(t \mathrm{Bu})$ & $\operatorname{Tyr}(t \mathrm{Bu})$ & Phe & $\operatorname{Thr}(t \mathrm{Bu})$ & $\operatorname{Tyr}(t \mathrm{Bu})$ & Phe & $\operatorname{Ser}(t \mathrm{Bu})$ & Lys(Boc) \\
\hline $4 g$ & $\operatorname{Thr}(t \mathrm{Bu})$ & $\operatorname{Tyr}(t \mathrm{Bu})$ & Phe & $\operatorname{Thr}(t \mathrm{Bu})$ & $\operatorname{Tyr}(t \mathrm{Bu})$ & $\operatorname{Tyr}(t \mathrm{Bu})$ & $\operatorname{Ser}(t \mathrm{Bu})$ & Lys(Boc) \\
\hline $4 h$ & $\mathrm{Glu}(t \mathrm{Bu})$ & $\operatorname{Ser}(t \mathrm{Bu})$ & Phe & $\operatorname{Thr}(t \mathrm{Bu})$ & $\operatorname{Tyr}(t \mathrm{Bu})$ & $\operatorname{Thr}(t \mathrm{Bu})$ & Lys(Boc) & Lys(Boc) \\
\hline $4 i$ & $\operatorname{Thr}(t \mathrm{Bu})$ & $\operatorname{Ser}(t \mathrm{Bu})$ & Leu & $\operatorname{Thr}(t \mathrm{Bu})$ & Val & $\operatorname{Thr}(t \mathrm{Bu})$ & $\operatorname{Ser}(t \mathrm{Bu})$ & Lys(Boc) \\
\hline $4 j$ & $\operatorname{Thr}(t \mathrm{Bu})$ & $\operatorname{Tyr}(t \mathrm{Bu})$ & Ile & $\operatorname{Thr}(t \mathrm{Bu})$ & Val & $\operatorname{Tyr}(t \mathrm{Bu})$ & $\operatorname{Ser}(t \mathrm{Bu})$ & Lys(Boc) \\
\hline $4 k$ & $\operatorname{Thr}(t \mathrm{Bu})$ & $\operatorname{Tyr}(t \mathrm{Bu})$ & Ile & $\operatorname{Thr}(t \mathrm{Bu})$ & $\operatorname{Thr}(t \mathrm{Bu})$ & $\operatorname{Tyr}(t \mathrm{Bu})$ & $\operatorname{Ser}(t \mathrm{Bu})$ & Lys(Boc) \\
\hline 41 & $\operatorname{Thr}(t \mathrm{Bu})$ & $\operatorname{Tyr}(t \mathrm{Bu})$ & $\operatorname{Ser}(t \mathrm{Bu})$ & $\operatorname{Thr}(t \mathrm{Bu})$ & Val & $\operatorname{Tyr}(t \mathrm{Bu})$ & $\operatorname{Ser}(t \mathrm{Bu})$ & Lys(Boc) \\
\hline $4 m$ & $\operatorname{Thr}(t \mathrm{Bu})$ & $\operatorname{Tyr}(t \mathrm{Bu})$ & $\operatorname{Ser}(t \mathrm{Bu})$ & $\operatorname{Thr}(t \mathrm{Bu})$ & $\operatorname{Thr}(t \mathrm{Bu})$ & $\operatorname{Tyr}(t \mathrm{Bu})$ & $\operatorname{Ser}(t \mathrm{Bu})$ & Lys(Boc) \\
\hline $4 n$ & Phe & $\operatorname{Tyr}(t \mathrm{Bu})$ & $\operatorname{Ser}(t \mathrm{Bu})$ & $\operatorname{Thr}(t \mathrm{Bu})$ & $\operatorname{Thr}(t \mathrm{Bu})$ & Phe & $\operatorname{Tyr}(t \mathrm{Bu})$ & Lys(Boc) \\
\hline
\end{tabular}




\section{Representative synthesis of protected linear peptide 4a (H-Thr(tBu)-Ser(tBu)-Phe-}

$\left.\operatorname{Thr}(t \mathrm{Bu})-\operatorname{Tyr}(t \mathrm{Bu})-\operatorname{Thr}(t \mathrm{Bu})-\operatorname{Ser}(t \mathrm{Bu})-{ }^{\delta} \operatorname{Orn}(\alpha-B o c)-H a o-L y s(B o c)-H a o-{ }^{\delta} \operatorname{Orn}(\alpha-B o c)-O H\right)$.

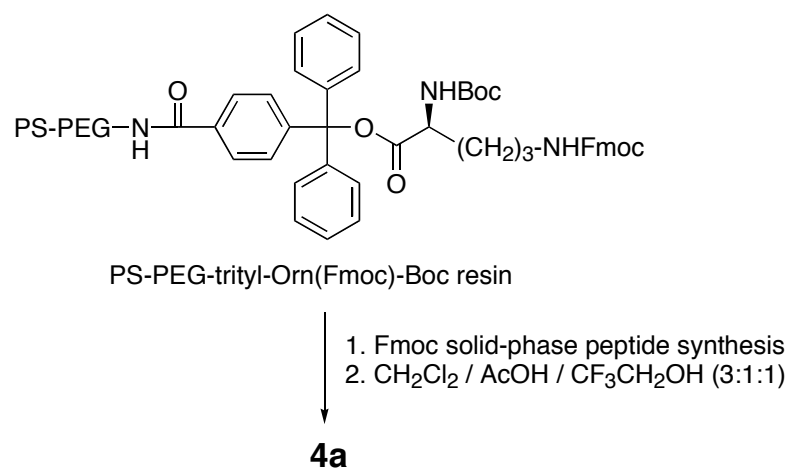

PS-PEG-trityl-Orn(Fmoc)-Boc resin (0.44 g, $0.145 \mathrm{mmol} / \mathrm{g}$ loading, $0.064 \mathrm{mmol})$ was washed with $\mathrm{CH}_{2} \mathrm{Cl}_{2}(3 \times 5 \mathrm{~mL})$ and DMF $(3 \times 5 \mathrm{~mL})$ and was submitted to cycles of Fmoc solid-phase peptide synthesis on the automatic continuous peptide synthesizer using amino acid building blocks: Fmoc*-Hao-OH, ${ }^{6}$ Fmoc-Lys(Boc)-OH, Fmoc*-Hao-OH, Boc-Orn(Fmoc)-OH, Fmoc-Ser( $t \mathrm{Bu})-\mathrm{OH}$, Thr $(t \mathrm{Bu})-\mathrm{OH}$, Fmoc-Tyr $(t \mathrm{Bu})-\mathrm{OH}$, Fmoc-Thr( $t \mathrm{Bu})-\mathrm{OH}$, Fmoc-Phe-OH, Fmoc-Ser $(t \mathrm{Bu})-\mathrm{OH}$, and Fmoc-Thr $(t \mathrm{Bu})-\mathrm{OH}$. Each coupling consisted of: (a) Fmoc-deprotection with $20 \%$ piperidine in DMF (30 mL, $5 \mathrm{~min}$ ); (b) washing with DMF; (c) coupling of the amino acid ( $0.40 \mathrm{mmol}, 6.2$ equiv) in the presence of HBTU (152 mg, $0.40 \mathrm{mmol}, 6.2$ equiv) and HOBt (54 mg, $0.40 \mathrm{mmol}, 6.2$ equiv) and DIPEA (130 $\mu \mathrm{L}, 0.80 \mathrm{mmol}, 12.5$ equiv), or for Hao, with HOAt (54 mg, $0.40 \mathrm{mmol}, 6.2$ equiv) and DIC (63 $\mu \mathrm{L}, 0.4 \mathrm{mmol}, 6.2$ equiv) ${ }^{7}$ (d) washing with DMF. Coupling reactions of the amino acids Thr, Ser, Phe, and Tyr were performed for $2 \mathrm{~h}$. Hao was coupled twice, first for $8 \mathrm{~h}$, then for $2 \mathrm{~h}$, to ensure complete coupling. Orn, and Lys were also coupled twice, each time for $2 \mathrm{~h}$. The $N$-terminus amine was Fmoc-deprotected with $20 \%$ piperidine in DMF (30 mL, $5 \mathrm{~min}$ ), and the resin was washed with DMF. The resin was then transferred to a plastic solid-phase synthesis reaction vessel and was washed with $\mathrm{CH}_{2} \mathrm{Cl}_{2}(3 \times 5$ $\mathrm{mL})$ and treated with the cleavage cocktail $\mathrm{AcOH}-\mathrm{CH}_{2} \mathrm{Cl}_{2}-\mathrm{CF}_{3} \mathrm{CH}_{2} \mathrm{OH}(1: 3: 1 \mathrm{v} / \mathrm{v}, 2 \times 8 \mathrm{~mL}, 2 \mathrm{~h}$ each time). ${ }^{4}$ The fractions were collected and concentrated under vacuum. The remaining acetic acid was azeotropically removed from the product by successive co-evaporation with hexanes

6 Nowick, J. S.; Chung, D. M.; Maitra, K.; Maitra, S.; Stigers, K. D.; Sun, Y. J. Am. Chem. Soc. 2000, 122, 76547661.

7 Using 4 equiv of amino acids, 4 equiv of coupling reagents, and 8 equiv of DIPEA gives similar yields of the linear peptide. 
(20 mL), $\mathrm{CH}_{2} \mathrm{Cl}_{2}$-hexanes $(1 / 2 \mathrm{v} / \mathrm{v}, 30 \mathrm{~mL})$, and hexanes $(20 \mathrm{~mL})$ to give $128 \mathrm{mg}$ crude linear peptide 4a (crude yield $=88 \%$ ). The crude linear peptide $4 \mathbf{a}$ was directly cyclized without further purification. 


\section{Protected cyclic peptides 5:}

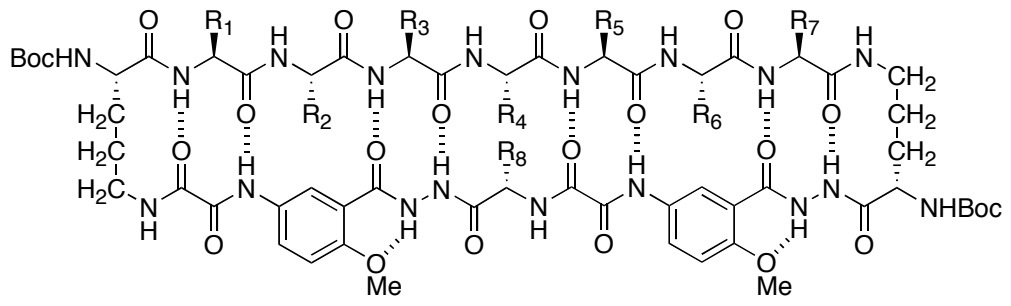

\begin{tabular}{|c|c|c|c|c|c|c|c|c|}
\hline Peptide & $R_{1}$ & $\mathbf{R}_{2}$ & $\mathbf{R}_{3}$ & $\mathbf{R}_{4}$ & $\mathbf{R}_{5}$ & $\mathbf{R}_{6}$ & $\mathbf{R}_{7}$ & $\mathbf{R}_{8}$ \\
\hline $5 a$ & $\operatorname{Thr}(t \mathrm{Bu})$ & $\operatorname{Ser}(t \mathrm{Bu})$ & Phe & $\operatorname{Thr}(t \mathrm{Bu})$ & $\operatorname{Tyr}(t \mathrm{Bu})$ & $\operatorname{Thr}(t \mathrm{Bu})$ & $\operatorname{Ser}(t \mathrm{Bu})$ & Lys(Boc) \\
\hline $5 \mathbf{b}$ & $\operatorname{Thr}(t \mathrm{Bu})$ & $\operatorname{Ser}(t \mathrm{Bu})$ & Phe & $\operatorname{Glu}(t \mathrm{Bu})$ & $\operatorname{Tyr}(t \mathrm{Bu})$ & $\operatorname{Thr}(t \mathrm{Bu})$ & $\operatorname{Ser}(t \mathrm{Bu})$ & Lys(Boc) \\
\hline $5 c$ & $\operatorname{Thr}(t \mathrm{Bu})$ & $\operatorname{Ser}(t \mathrm{Bu})$ & Phe & Leu & $\operatorname{Tyr}(t \mathrm{Bu})$ & $\operatorname{Thr}(t \mathrm{Bu})$ & $\operatorname{Ser}(t \mathrm{Bu})$ & Lys(Boc) \\
\hline $5 d$ & $\operatorname{Thr}(t \mathrm{Bu})$ & $\operatorname{Ser}(t \mathrm{Bu})$ & Phe & $\operatorname{Tyr}(t \mathrm{Bu})$ & $\operatorname{Tyr}(t \mathrm{Bu})$ & $\operatorname{Thr}(t \mathrm{Bu})$ & $\operatorname{Ser}(t \mathrm{Bu})$ & Lys(Boc) \\
\hline $5 e$ & $\operatorname{Thr}(t \mathrm{Bu})$ & Leu & Phe & $\operatorname{Thr}(t \mathrm{Bu})$ & $\operatorname{Tyr}(t \mathrm{Bu})$ & Val & $\operatorname{Ser}(t \mathrm{Bu})$ & Lys(Boc) \\
\hline $5 f$ & $\operatorname{Thr}(t \mathrm{Bu})$ & $\operatorname{Tyr}(t \mathrm{Bu})$ & Phe & $\operatorname{Thr}(t \mathrm{Bu})$ & $\operatorname{Tyr}(t \mathrm{Bu})$ & Phe & $\operatorname{Ser}(t \mathrm{Bu})$ & Lys(Boc) \\
\hline $5 g$ & $\operatorname{Thr}(t \mathrm{Bu})$ & $\operatorname{Tyr}(t \mathrm{Bu})$ & Phe & $\operatorname{Thr}(t \mathrm{Bu})$ & $\operatorname{Tyr}(t \mathrm{Bu})$ & $\operatorname{Tyr}(t \mathrm{Bu})$ & $\operatorname{Ser}(t \mathrm{Bu})$ & Lys(Boc) \\
\hline $5 \mathrm{~h}$ & $\operatorname{Glu}(t \mathrm{Bu})$ & $\operatorname{Ser}(t \mathrm{Bu})$ & Phe & $\operatorname{Thr}(t \mathrm{Bu})$ & $\operatorname{Tyr}(t \mathrm{Bu})$ & $\operatorname{Thr}(t \mathrm{Bu})$ & Lys(Boc) & Lys(Boc) \\
\hline $5 i$ & $\operatorname{Thr}(t \mathrm{Bu})$ & $\operatorname{Ser}(t \mathrm{Bu})$ & Leu & $\operatorname{Thr}(t \mathrm{Bu})$ & Val & $\operatorname{Thr}(t \mathrm{Bu})$ & $\operatorname{Ser}(t \mathrm{Bu})$ & Lys(Boc) \\
\hline $5 \mathbf{j}$ & $\operatorname{Thr}(t \mathrm{Bu})$ & $\operatorname{Tyr}(t \mathrm{Bu})$ & Ile & $\operatorname{Thr}(t \mathrm{Bu})$ & Val & $\operatorname{Tyr}(t \mathrm{Bu})$ & $\operatorname{Ser}(t \mathrm{Bu})$ & Lys(Boc) \\
\hline $5 \mathbf{k}$ & $\operatorname{Thr}(t \mathrm{Bu})$ & $\operatorname{Tyr}(t \mathrm{Bu})$ & Ile & $\operatorname{Thr}(t \mathrm{Bu})$ & $\operatorname{Thr}(t \mathrm{Bu})$ & $\operatorname{Tyr}(t \mathrm{Bu})$ & $\operatorname{Ser}(t \mathrm{Bu})$ & Lys(Boc) \\
\hline 51 & $\operatorname{Thr}(t \mathrm{Bu})$ & $\operatorname{Tyr}(t \mathrm{Bu})$ & $\operatorname{Ser}(t \mathrm{Bu})$ & $\operatorname{Thr}(t \mathrm{Bu})$ & Val & $\operatorname{Tyr}(t \mathrm{Bu})$ & $\operatorname{Ser}(t \mathrm{Bu})$ & Lys(Boc) \\
\hline $5 \mathrm{~m}$ & $\operatorname{Thr}(t \mathrm{Bu})$ & $\operatorname{Tyr}(t \mathrm{Bu})$ & $\operatorname{Ser}(t \mathrm{Bu})$ & $\operatorname{Thr}(t \mathrm{Bu})$ & $\operatorname{Thr}(t \mathrm{Bu})$ & $\operatorname{Tyr}(t \mathrm{Bu})$ & $\operatorname{Ser}(t \mathrm{Bu})$ & Lys(Boc) \\
\hline $5 n$ & Phe & $\operatorname{Tyr}(t \mathrm{Bu})$ & $\operatorname{Ser}(t \mathrm{Bu})$ & $\operatorname{Thr}(t \mathrm{Bu})$ & $\operatorname{Thr}(t \mathrm{Bu})$ & Phe & $\operatorname{Tyr}(t \mathrm{Bu})$ & Lys(Boc) \\
\hline
\end{tabular}

Representative preparation of protected cyclic peptide 5a $\left(\operatorname{cyclo}\left[{ }^{\delta} \operatorname{Orn}(\alpha-\operatorname{Boc})\right.\right.$ $\operatorname{Thr}(t \mathrm{Bu})-\operatorname{Ser}(t \mathrm{Bu})-\mathrm{Phe}-\operatorname{Thr}(t \mathrm{Bu})-\operatorname{Tyr}(t \mathrm{Bu})-\operatorname{Thr}(t \mathrm{Bu})-\operatorname{Ser}(t \mathrm{Bu})-{ }^{\delta} \operatorname{Orn}(\alpha-\mathrm{Boc})-\mathrm{Hao}-\mathrm{Lys}(\mathrm{Boc})-$ Hao]).

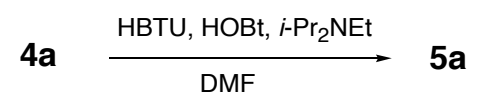

A one-liter one-necked round-bottomed flask equipped with a magnetic stirring bar, and a pressure-equalizing funnel (connected to $\mathrm{N}_{2}$ gas inlet) was charged with a solution of HBTU (115 mg, $0.30 \mathrm{mmol}, 6.2$ equiv), HOBt (42 mg, $0.31 \mathrm{mmol}, 6.4$ equiv), and DIPEA (110 $\mu \mathrm{L}$, 
$0.67 \mathrm{mmol}, 14.0$ equiv) in DMF (100 mL). A solution of crude peptide 4a (110 mg, $0.048 \mathrm{mmol}$ based on $100 \%$ purity) in DMF (100 mL) was added to the flask via the pressure-equalizing addition funnel over $4 \mathrm{~h}$, and the reaction solution was subsequently stirred for $16 \mathrm{~h}$. The solvent was then removed under vacuum, and the resulting residue was stirred vigorously with water (50 $\mathrm{mL})$ and $\mathrm{CH}_{2} \mathrm{Cl}_{2}(200 \mathrm{~mL})$ for $3 \mathrm{~h}$. The organic layer was separated and was washed with water $(50 \mathrm{~mL})$. The organic layer was concentrated under vacuum to give crude protected cyclic peptide 5a, which was directly taken to the next step without further purification. 


\section{Cyclic peptides 3:}

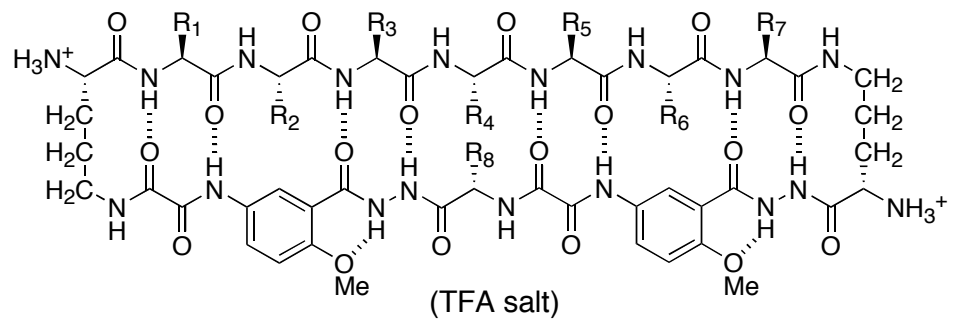

\begin{tabular}{|c|c|c|c|c|c|c|c|c|}
\hline Peptide & $R_{1}$ & $\mathbf{R}_{2}$ & $\mathbf{R}_{3}$ & $\mathbf{R}_{4}$ & $\mathbf{R}_{5}$ & $\mathbf{R}_{6}$ & $\mathbf{R}_{7}$ & $\mathbf{R}_{8}$ \\
\hline $3 \mathbf{a}$ & Thr & Ser & Phe & Thr & Tyr & Thr & Ser & $\overline{\text { Lys }}$ \\
\hline $3 b$ & Thr & Ser & Phe & Glu & Tyr & Thr & Ser & Lys \\
\hline $3 c$ & Thr & Ser & Phe & Leu & Tyr & Thr & Ser & Lys \\
\hline 3d & Thr & Ser & Phe & Tyr & Tyr & Thr & Ser & Lys \\
\hline $3 e$ & Thr & Leu & Phe & Thr & Tyr & Val & Ser & Lys \\
\hline $3 f$ & Thr & Tyr & Phe & Thr & Tyr & Phe & Ser & Lys \\
\hline $3 g$ & Thr & Tyr & Phe & Thr & Tyr & Tyr & Ser & Lys \\
\hline $3 h$ & Glu & Ser & Phe & Thr & Tyr & Thr & Lys & Lys \\
\hline $3 \mathbf{i}$ & Thr & Ser & Leu & Thr & Val & Thr & Ser & Lys \\
\hline 3j & Thr & Tyr & Ile & Thr & Val & Tyr & Ser & Lys \\
\hline $3 \mathbf{k}$ & Thr & Tyr & Ile & Thr & Thr & Tyr & Ser & Lys \\
\hline 31 & Thr & Tyr & Ser & Thr & Val & Tyr & Ser & Lys \\
\hline $3 m$ & Thr & Tyr & Ser & Thr & Thr & Tyr & Ser & Lys \\
\hline $3 n$ & Phe & Tyr & Ser & Thr & Thr & Phe & Tyr & Lys \\
\hline
\end{tabular}

Representative preparation of cyclic peptide 3a $\left(\mathrm{Cyclo}^{\delta}\left[{ }^{\delta} \mathrm{Orn}-\mathrm{Thr}-\mathrm{Ser}-\mathrm{Phe}-\mathrm{Thr}-\mathrm{Tyr}-\right.\right.$ Thr-Ser- ${ }^{\delta}$ Orn-Hao-Lys-Hao] trifluoroacetate salt).

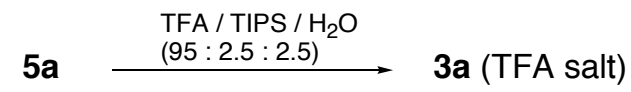

Crude peptide 5a was stirred with TFA-TIPS- $\mathrm{H}_{2} \mathrm{O}(95: 2.5: 2.5,20 \mathrm{~mL})$ for $3 \mathrm{~h}$, and the solvent was removed under vacuum. The resulting residue was dissolved in methanol $(50 \mathrm{~mL})$ 
and heated at $50{ }^{\circ} \mathrm{C}$ for $1 \mathrm{~h}^{8}{ }^{8}$ The mixture was then concentrated under vacuum. The remaining material was dissolved in $30 \mathrm{~mL}$ of water and the solution was lyophilized to give $175 \mathrm{mg}$ crude cyclic peptide 3a. The crude peptide $3 \mathbf{a}$ was then dissolved in water- $\mathrm{CH}_{3} \mathrm{CN}(5 / 1 \mathrm{v} / \mathrm{v}, 3 \mathrm{~mL})$ and purified by RP-HPLC to afford $34 \mathrm{mg}$ pure peptide 3a in overall yield of $18 \%$, based on the loading of the resin with Boc-Orn(Fmoc)-OH. The purity of peptide determined by analytical RP-HPLC ( $\mathrm{R}_{\mathrm{t}} 16.6 \mathrm{~min}$; linear gradient 10 to $50 \% \mathrm{~B}$ in $20 \mathrm{~min}$ ) and LRMS (ESI): $\mathrm{m} / \mathrm{z}$ for $\mathrm{C}_{72} \mathrm{H}_{100} \mathrm{~N}_{19} \mathrm{O}_{24}[\mathrm{M}+\mathrm{H}]^{+}$calcd. 1614.72, found 1614.94.

\section{Yields for other macrocyclic peptides 3}

Similar synthesis of other macrocyclic peptides $\mathbf{3}$ gave product in overall yields summarized below:

\begin{tabular}{cc}
\hline Peptide & $\begin{array}{c}\text { Yield } \\
(\%)\end{array}$ \\
\hline 3b & 14 \\
3c & 10 \\
3d & 5 \\
3e & 8 \\
3f & 12 \\
\hline 3g & 28 \\
3h & 13 \\
3i & 17 \\
3j & 19 \\
3k & 25 \\
31 & 13 \\
3m & 12 \\
3n & 26 \\
\hline &
\end{tabular}

8 Heating with methanol converts the trifluoroacetyl esters of hydroxy groups of Thr and Ser residues, which form during the deprotection of the peptide, to free hydroxy groups. 


\section{Control acyclic peptide 6 (H-Orn( $\delta$-Ac)-Thr-Ser-Phe-Thr-Tyr-Thr-Ser- ${ }^{\delta}$ Orn-NH}

trifluoroacetate salt).

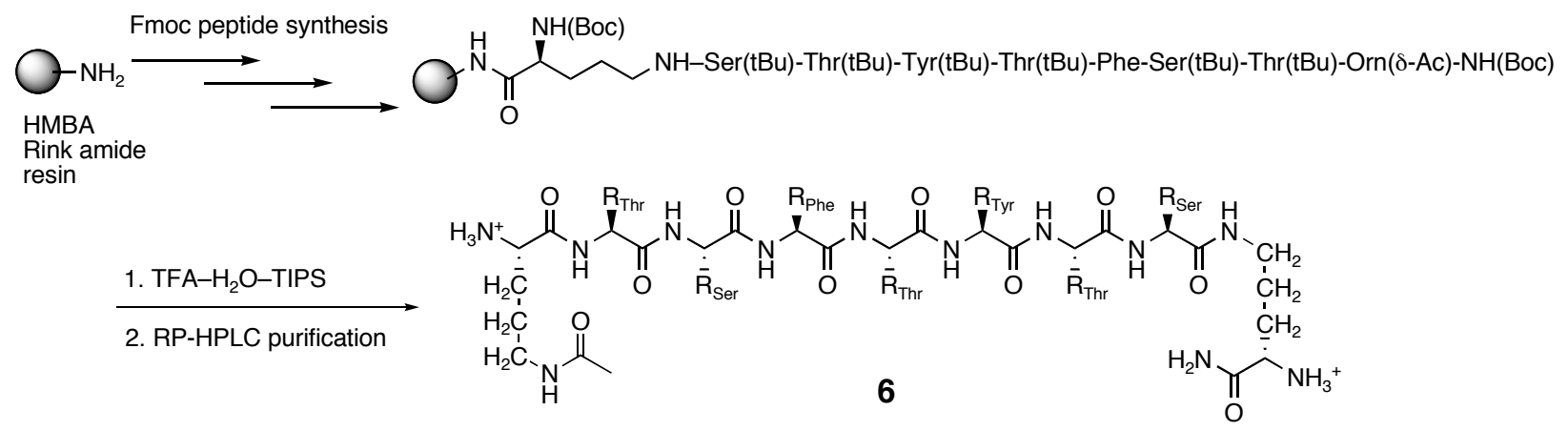

A 100-mL glass solid-phase synthesis column was charged with HMBA Rink amide resin (205 mg, $0.15 \mathrm{mmol}$ ), and the resin was washed with DMF (10 mL, $10 \mathrm{~min}$ ) and allowed to swell with $\mathrm{CH}_{2} \mathrm{Cl}_{2}(10 \mathrm{~mL}, 5 \mathrm{~min})$ and $\mathrm{DMF}(3 \times 10 \mathrm{~mL}, 5 \mathrm{~min}$ each time $)$. The resin was then subjected to nine cycles of Fmoc solid-phase synthesis with amino acid building blocks: BocOrn(Fmoc)-OH, Fmoc-Ser( $t \mathrm{Bu})-\mathrm{OH}, \operatorname{Thr}(t \mathrm{Bu})-\mathrm{OH}, \operatorname{Fmoc}-\mathrm{Tyr}(t \mathrm{Bu})-\mathrm{OH}, \operatorname{Fmoc}-\mathrm{Thr}(t \mathrm{Bu})-\mathrm{OH}$, Fmoc-Phe-OH, Fmoc-Ser( $t \mathrm{Bu})-\mathrm{OH}$, Fmoc-Thr( $t \mathrm{Bu})-\mathrm{OH}$, and Boc-Orn(Fmoc)-OH. Each cycle consisted of: (a) Fmoc-deprotection with $20 \%$ piperidine in DMF (25 mL, $20 \mathrm{~min}$ ); (b) washing with DMF, and $\mathrm{CH}_{2} \mathrm{Cl}_{2}$; (c) coupling of the amino acid (0.60 mmol, 4.0 equiv, $0.12 \mathrm{M}$ in DMF) in the presence of HBTU (230 mg, $0.60 \mathrm{mmol}, 4.0$ equiv) and HOBt ( $88 \mathrm{mg}, 0.60 \mathrm{mmol}, 4.0$ equiv) and DIPEA (250 $\mu \mathrm{L}, 1.5 \mathrm{mmol}, 10.0$ equiv), (d) washing with $\mathrm{DMF}$ and $\mathrm{CH}_{2} \mathrm{Cl}_{2}$, (e) capping of unreacted $N$-terminus peptides with $\mathrm{Ac}_{2} \mathrm{O}-\mathrm{CH}_{2} \mathrm{Cl}_{2}-\mathrm{DIPEA}(1: 9: 1,11 \mathrm{~mL}, 10 \mathrm{~min})$, and (f) washing with $\mathrm{CH}_{2} \mathrm{Cl}_{2}$ and DMF. The coupling of first amino acid, however, was performed with $340 \mathrm{mg}$ of Boc-Orn(Fmoc)-OH (0.75 mmol, 5.0 equiv, 0.15 M in DMF) in the presence of HOBt (110 mg, $0.75 \mathrm{mmol}, 5.0$ equiv) and DIC (120 $\mu \mathrm{L}, 0.75 \mathrm{mmol}, 5.0$ equiv). Each amino acid was coupled for $4 \mathrm{~h}$, and the completion of coupling and deprotection steps were checked with the Kaiser test. ${ }^{9}$ The peptide was cleaved from the resin with TFA- $\mathrm{H}_{2} \mathrm{O}-\mathrm{TIPS}$ (95:2.5:2.5 v/v, $20 \mathrm{~mL}, 12 \mathrm{~h})$, and the volatile material was removed under vacuum. The resulting residue was dissolved in methanol $(25 \mathrm{~mL})$ and heated at $50{ }^{\circ} \mathrm{C}$ for $2 \mathrm{~h}$. The solvent was removed under vacuum. The remaining material was dissolved in $30 \mathrm{~mL}$ of water and lyophilizied to give crude peptide $\mathbf{6}$. Crude peptide $\mathbf{6}$ was then purified by RP-HPLC to afford 42

9 Kaiser, E.; Colescott, R. L.; Bossinger, C. D.; Cook, P. I. Anal. Biochem. 1970, 4, 595. 
mg $(22 \%)$ of peptide $\mathbf{6}$. The purity of peptide $\mathbf{6}$ was determined by analytical RP-HPLC $\left(\mathrm{R}_{\mathrm{t}} 6.8\right.$ min; linear gradient 10 to $90 \% \mathrm{~B}$ in $20 \mathrm{~min}$ ) and LRMS (ESI): $m / z$ for $\mathrm{C}_{48} \mathrm{H}_{75} \mathrm{~N}_{12} \mathrm{O}_{16}[\mathrm{M}+\mathrm{H}]^{+}$ calcd. 1075.54, found 1075.57. 


\section{Analytical RP-HPLC of peptide 3a}

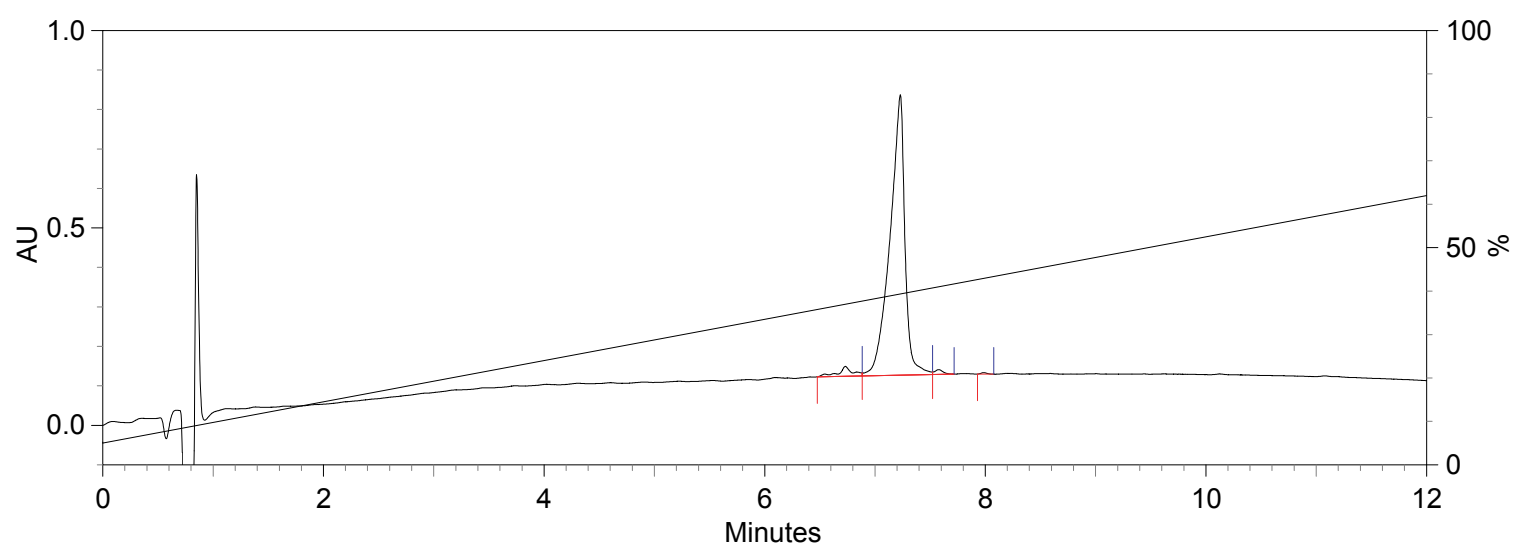

\begin{tabular}{|rrrrr|}
\hline Time & Area & Area \% & Height & Height \% \\
\hline 6.732 & 110589 & 3.17 & 12475 & 3.32 \\
7.230 & 3343151 & 95.70 & 355170 & 94.54 \\
7.578 & 32473 & 0.93 & 6106 & 1.63 \\
7.988 & 7333 & 0.21 & 1919 & 0.51 \\
\hline \multicolumn{5}{|c|}{} \\
Totals & 3493546 & 100.00 & 375670 & 100.00 \\
\hline
\end{tabular}

$\square=214 \mathrm{~nm} .80 \mathrm{SB}$ Zorbax column gradient $5 \%-100 \%$ solvent $B$ over 20 min. solvent A: Water (TFA 0.1\%); solvent $\mathrm{B}: \mathrm{CH}_{3} \mathrm{CN}$ (TFA $0.1 \%$ ) flow $=1 \mathrm{~mL} / \mathrm{min}$ 
LRMS(ESI) of peptide $\mathbf{3 a}$

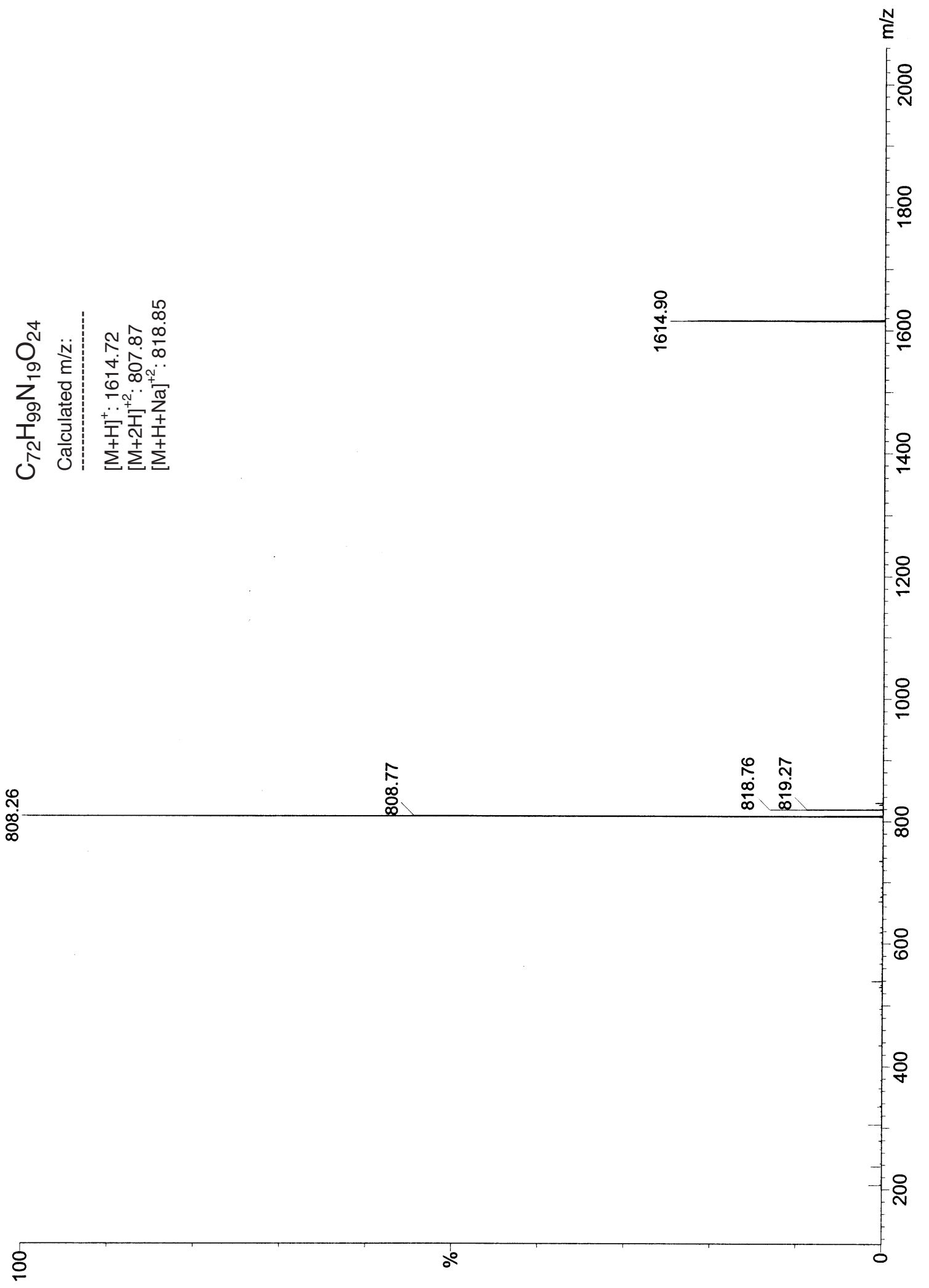


Analytical RP-HPLC of peptide $\mathbf{3 b}$

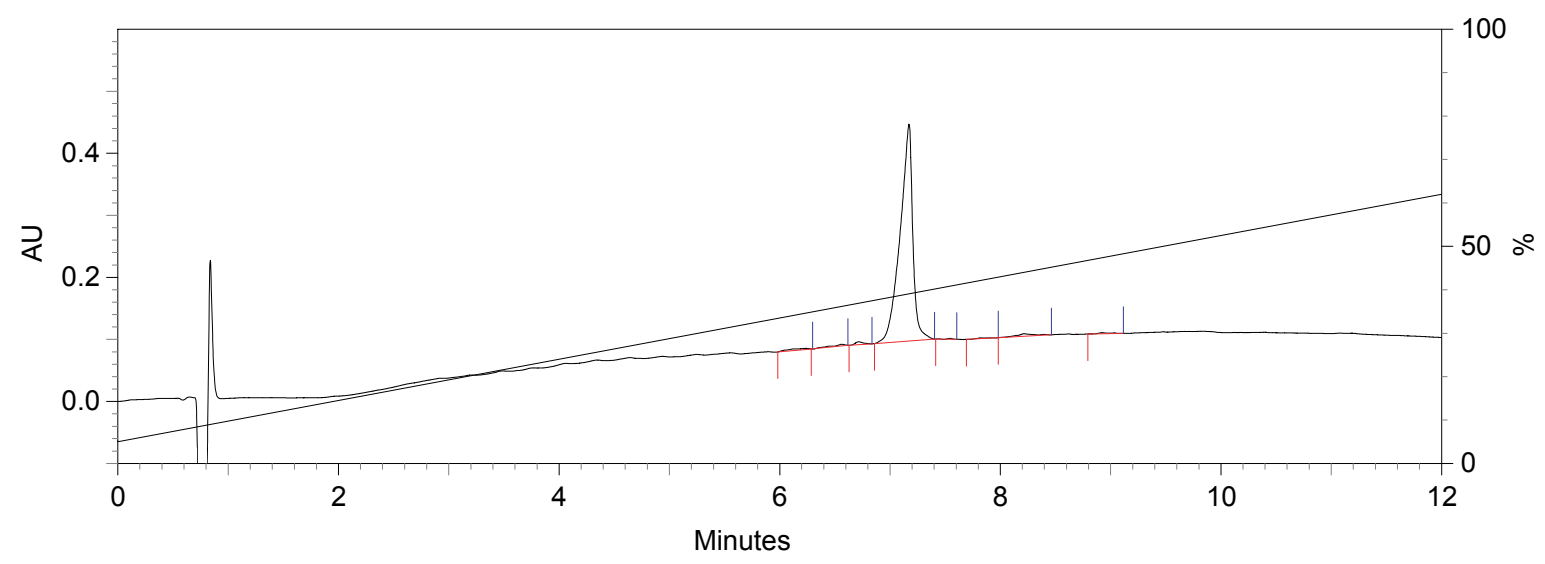

\begin{tabular}{|c|c|c|c|c|}
\hline Time & Area & Area \% & Height & Height $\%$ \\
\hline 6.232 & 14986 & 1.02 & 929 & 0.51 \\
\hline 6.560 & 8915 & 0.61 & 1284 & 0.70 \\
\hline 6.713 & 11809 & 0.81 & 2333 & 1.27 \\
\hline 7.172 & 1396313 & 95.26 & 174794 & 95.49 \\
\hline 7.543 & 2244 & 0.15 & 698 & 0.38 \\
\hline 7.697 & 3065 & 0.21 & 47 & 0.03 \\
\hline 8.217 & 22334 & 1.52 & 2061 & 1.13 \\
\hline 8.917 & 6187 & 0.42 & 894 & 0.49 \\
\hline \multirow[t]{2}{*}{ Totals } & & & & \\
\hline & 1465853 & 100.00 & 183040 & 100.00 \\
\hline
\end{tabular}

C= $214 \mathrm{~nm} .80$ SB Zorbax column

gradient $5 \%-100 \%$ solvent $B$ over 20 min.

solvent $\mathrm{A}$ : Water (TFA $0.1 \%$ ); solvent $\mathrm{B}: \mathrm{CH}_{3} \mathrm{CN}$ (TFA $0.1 \%$ )

flow $=1 \mathrm{~mL} / \mathrm{min}$ 
LRMS(ESI) of peptide $\mathbf{3 b}$

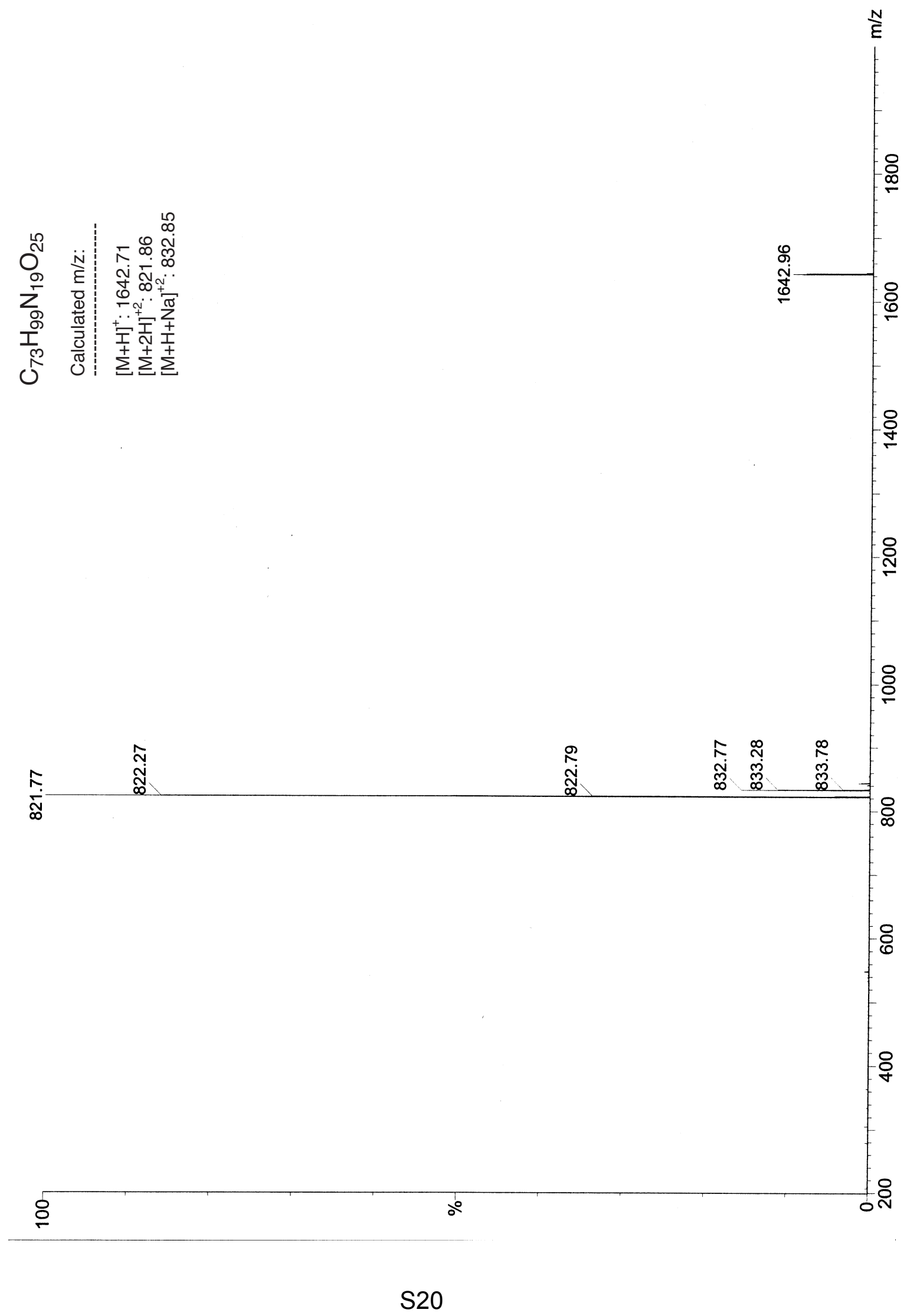




\section{Analytical RP-HPLC of peptide 3c}

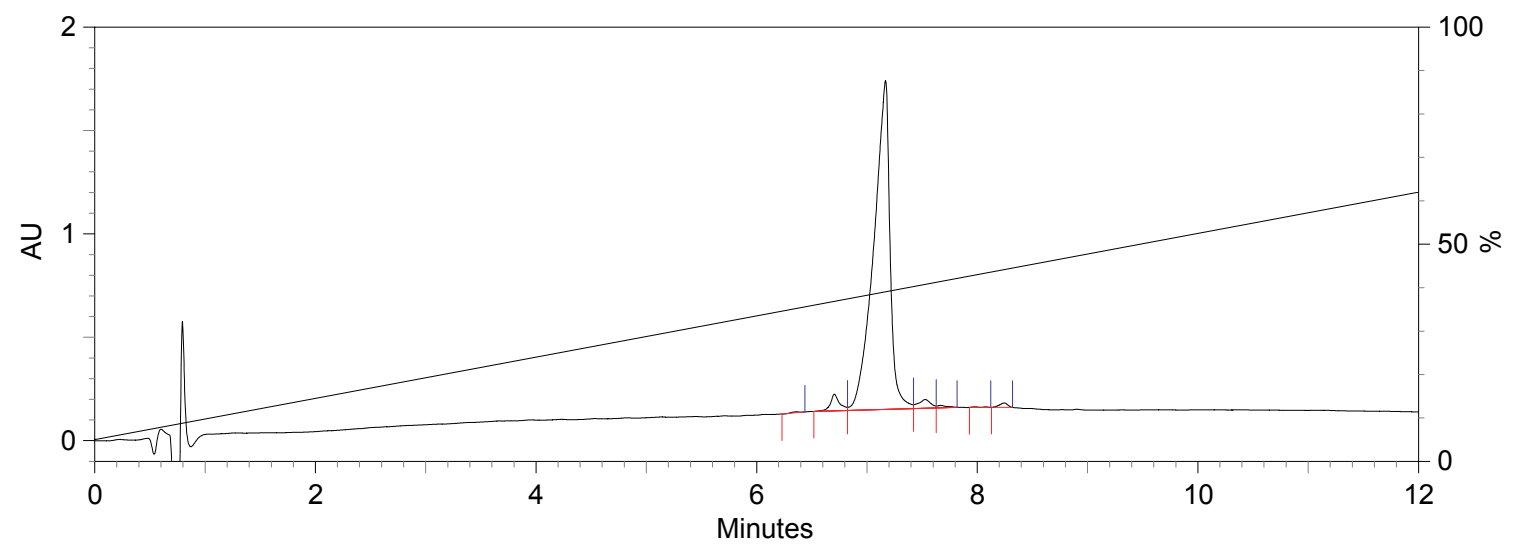

\begin{tabular}{|c|c|c|c|c|}
\hline Time & Area & Area $\%$ & Height & Height \% \\
\hline 6.358 & 9744 & 0.11 & 2284 & 0.26 \\
\hline 6.705 & 234622 & 2.71 & 40427 & 4.61 \\
\hline 7.168 & 8159934 & 94.10 & 795520 & 90.66 \\
\hline 7.528 & 164402 & 1.90 & 21042 & 2.40 \\
\hline 7.668 & 35506 & 0.41 & 5831 & 0.66 \\
\hline 7.972 & 10370 & 0.12 & 1495 & 0.17 \\
\hline 8.243 & 56911 & 0.66 & 10907 & 1.24 \\
\hline Totals & 8671489 & 100.00 & 877506 & 100.00 \\
\hline
\end{tabular}

C= $214 \mathrm{~nm} .80$ SB Zorbax column gradient $5 \%-100 \%$ solvent $B$ over 20 min. solvent $\mathrm{A}$ : Water (TFA $0.1 \%$ ); solvent $\mathrm{B}$ : $\mathrm{CH}_{3} \mathrm{CN}$ (TFA $0.1 \%$ ) flow $=1 \mathrm{~mL} / \mathrm{min}$ 
LRMS(ESI) of peptide $3 c$
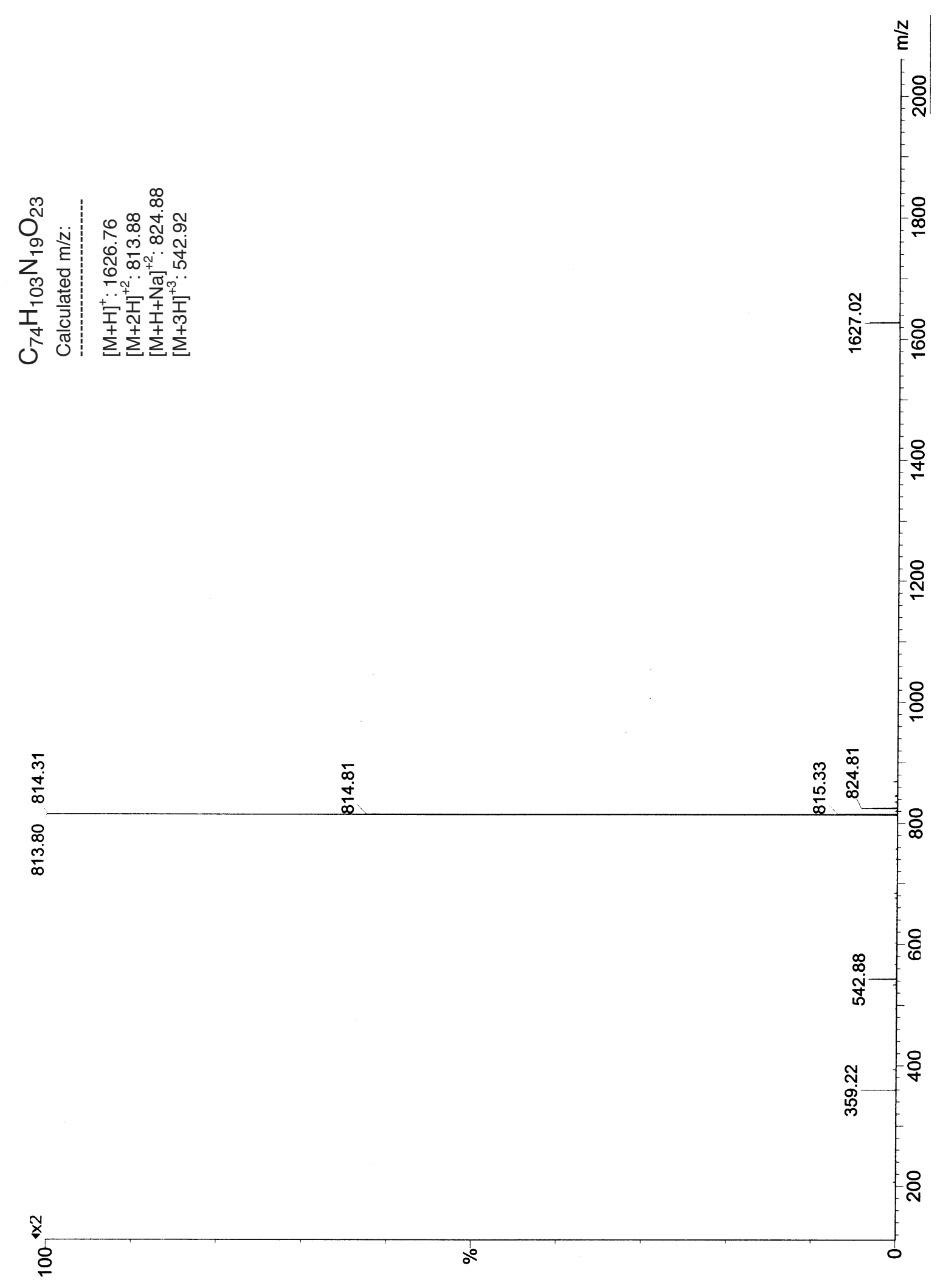


\section{Analytical RP-HPLC of peptide $\mathbf{3 d}$}

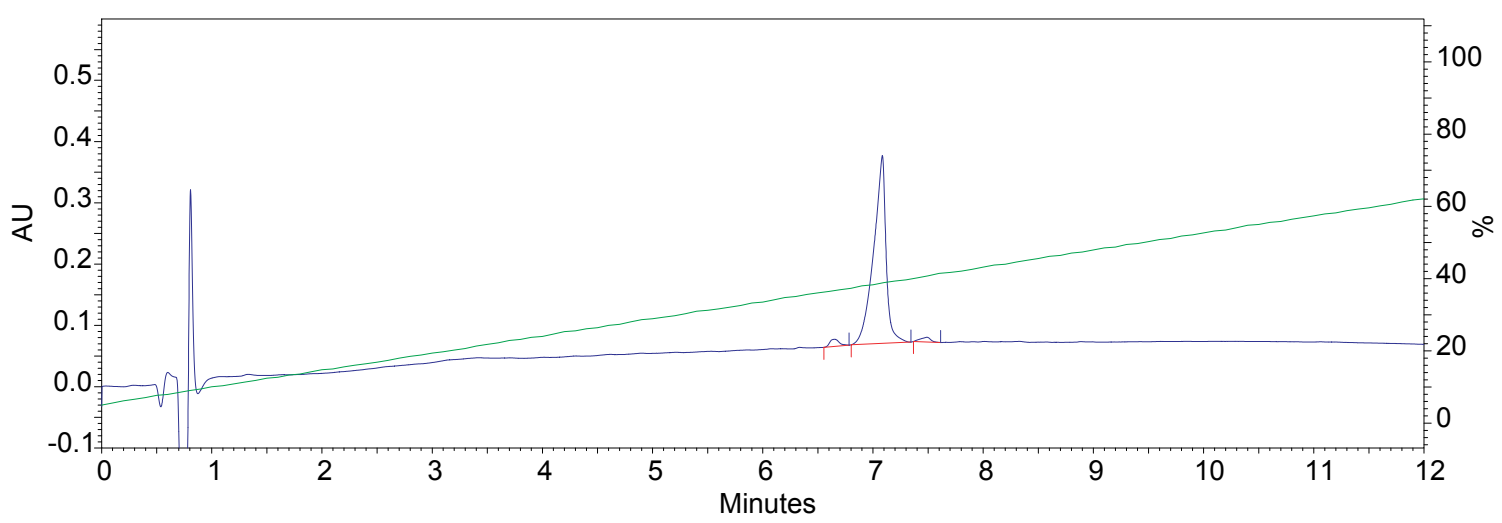

\begin{tabular}{rrrrr} 
Time & Area & Area \% & Height & Height \% \\
\hline 6.655 & 68546 & 2.71 & 11582 & 3.56 \\
7.085 & 2411081 & 95.31 & 306384 & 94.07 \\
7.490 & 50153 & 1.98 & 7735 & 2.37 \\
\hline \multicolumn{2}{|c|}{} & & & \\
\hline
\end{tabular}

C= $214 \mathrm{~nm} .80$ SB Zorbax column

gradient $5 \%-100 \%$ solvent $B$ over 20 min.

solvent $\mathrm{A}$ : Water (TFA $0.1 \%$ ); solvent $\mathrm{B}: \mathrm{CH}_{3} \mathrm{CN}$ (TFA $0.1 \%$ )

flow $=1 \mathrm{~mL} / \mathrm{min}$ 
LRMS(ESI) of peptide $\mathbf{3 d}$

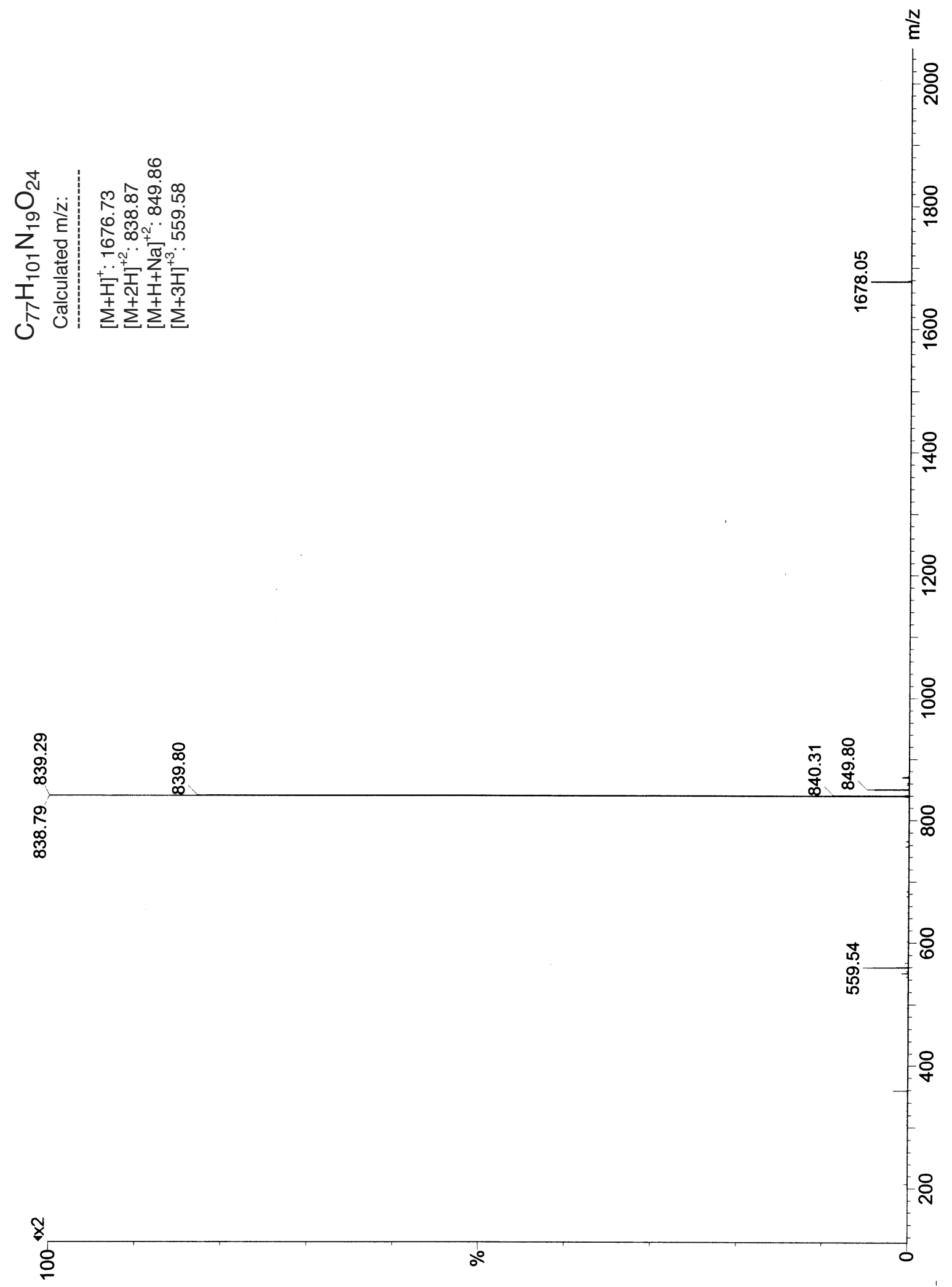




\section{Analytical RP-HPLC of peptide $3 e$}

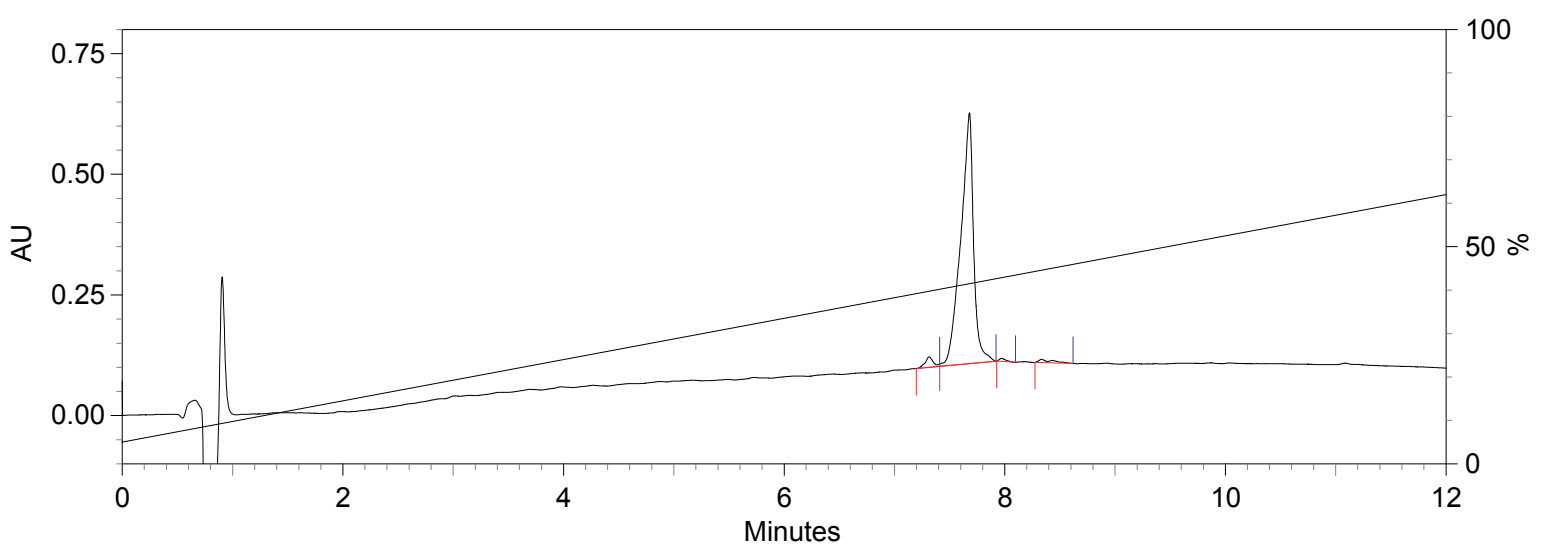

\begin{tabular}{rrrrr} 
Time & Area & Area \% & Height & Height \% \\
\hline 7.315 & 54021 & 2.46 & 10738 & 3.87 \\
7.680 & 2098740 & 95.60 & 259919 & 93.76 \\
7.970 & 12800 & 0.58 & 3174 & 1.15 \\
8.335 & 29728 & 1.35 & 3372 & 1.22 \\
\hline \multicolumn{7}{|c|}{2195289} & 100.00 & 277203 & 100.00 \\
\hline
\end{tabular}

$\square=214 \mathrm{~nm} .80$ SB Zorbax column gradient $5 \%-100 \%$ solvent $B$ over 20 min. solvent $\mathrm{A}$ : Water (TFA $0.1 \%$ ); solvent $\mathrm{B}: \mathrm{CH}_{3} \mathrm{CN}$ (TFA $0.1 \%$ ) flow $=1 \mathrm{~mL} / \mathrm{min}$ 
LRMS(ESI) of peptide $\mathbf{3 e}$
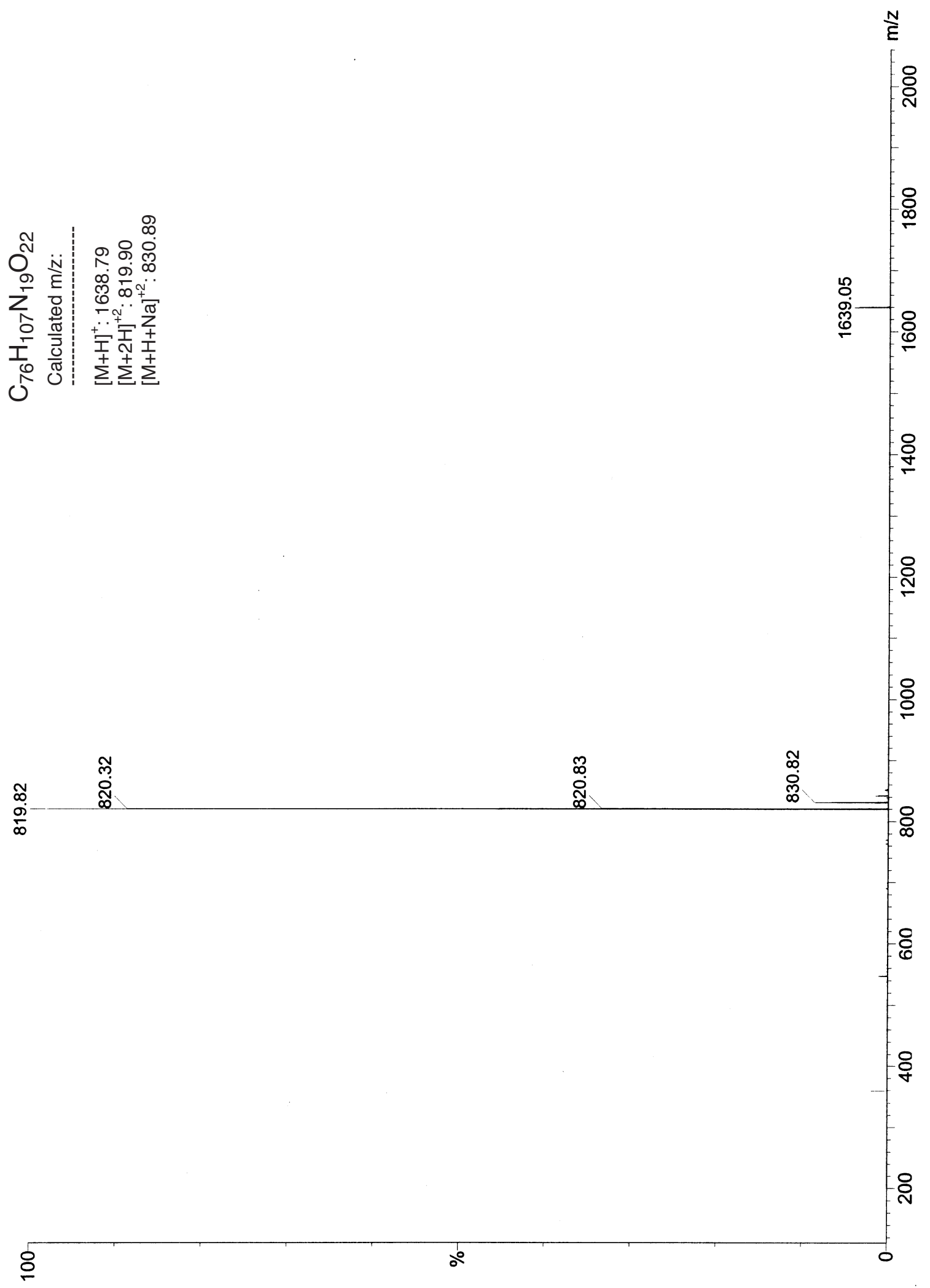


\section{Analytical RP-HPLC of peptide $\mathbf{3 f}$}

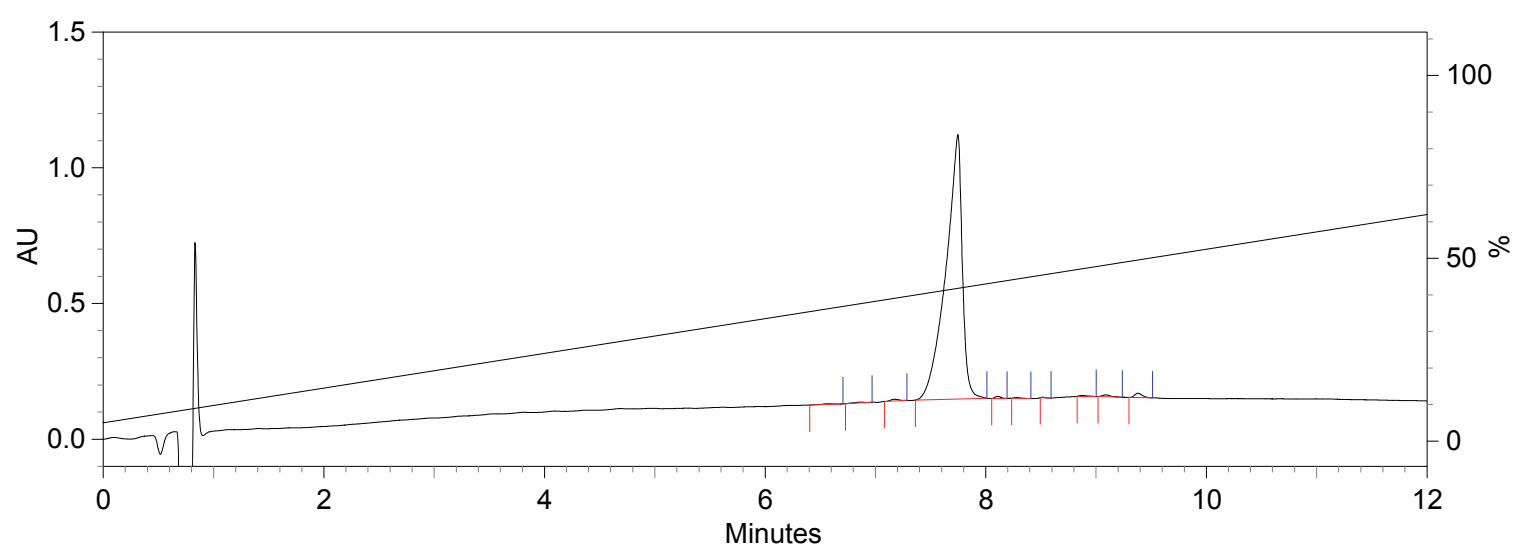

\begin{tabular}{|c|c|c|c|c|}
\hline Time & Area & Area \% & Height & Height \% \\
\hline 6.572 & 8563 & 0.17 & 1324 & 0.26 \\
\hline 6.872 & 8699 & 0.17 & 1382 & 0.27 \\
\hline 7.177 & 17192 & 0.33 & 3220 & 0.63 \\
\hline 7.747 & 5016429 & 97.68 & 487042 & 95.08 \\
\hline 8.107 & 13616 & 0.27 & 3954 & 0.77 \\
\hline 8.280 & 6875 & 0.13 & 1565 & 0.31 \\
\hline 8.513 & 1275 & 0.02 & 812 & 0.16 \\
\hline 8.870 & 8884 & 0.17 & 1731 & 0.34 \\
\hline 9.087 & 15684 & 0.31 & 3211 & 0.63 \\
\hline 9.380 & 38195 & 0.74 & 8000 & 1.56 \\
\hline \multirow[t]{2}{*}{ Totals } & & & & \\
\hline & 5135412 & 100.00 & 512241 & 100.00 \\
\hline
\end{tabular}

C= $214 \mathrm{~nm} .80$ SB Zorbax column

gradient $5 \%-100 \%$ solvent $B$ over 20 min.

solvent $\mathrm{A}$ : Water (TFA $0.1 \%$ ); solvent $\mathrm{B}$ : $\mathrm{CH}_{3} \mathrm{CN}$ (TFA $0.1 \%$ )

flow $=1 \mathrm{~mL} / \mathrm{min}$ 


\section{LRMS(ESI) of peptide $\mathbf{3 f}$}
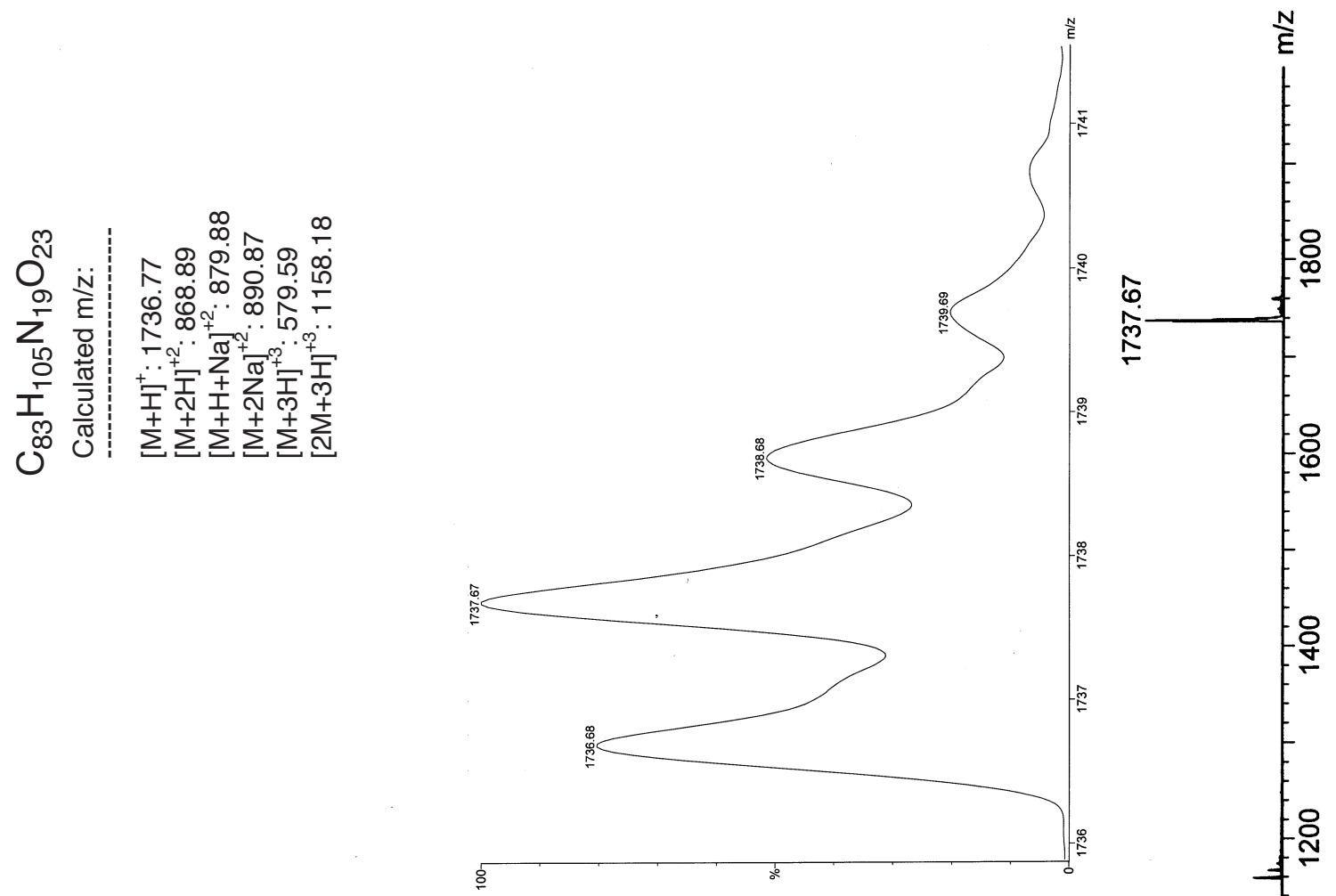

ळ

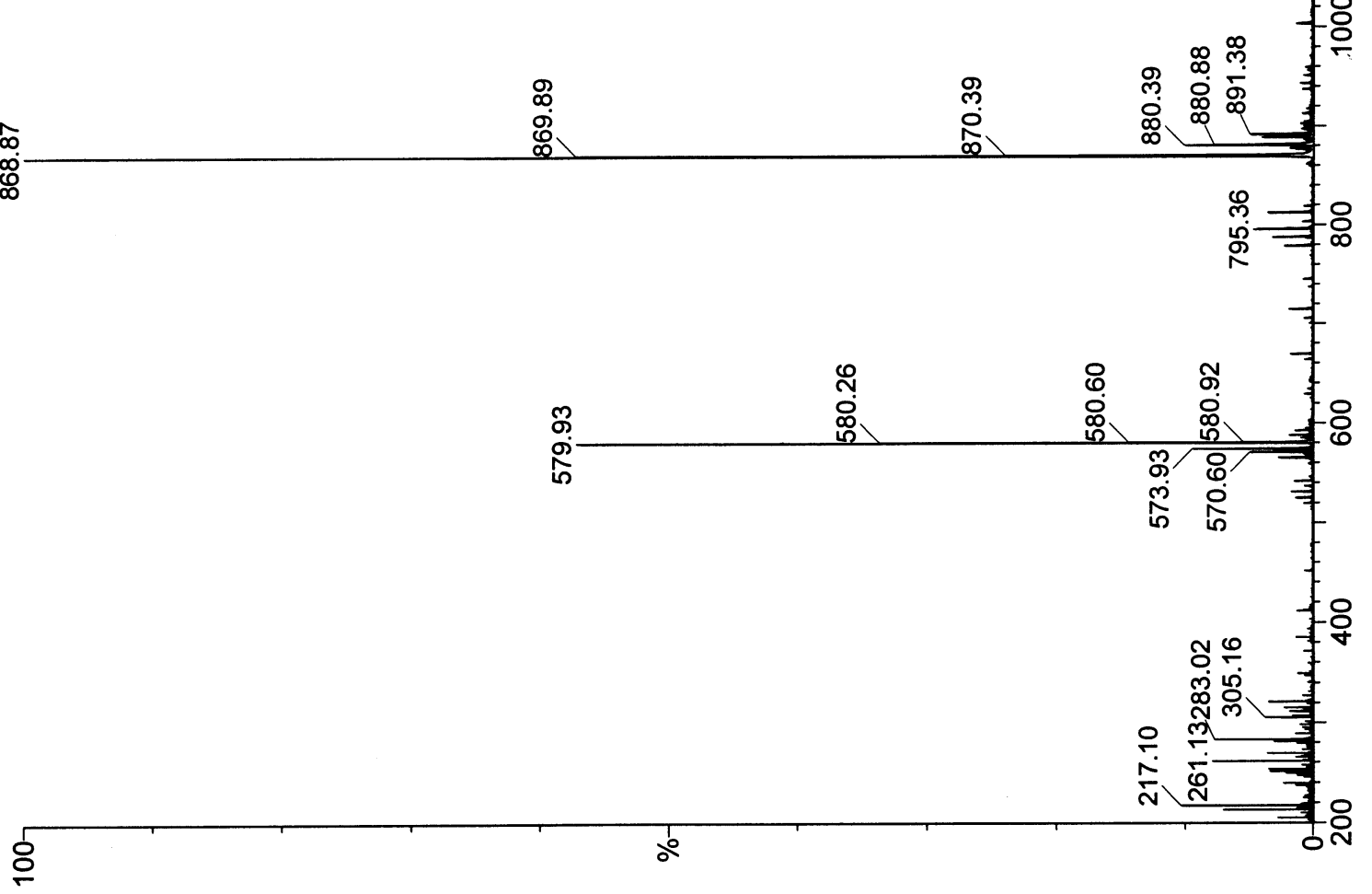




\section{Analytical RP-HPLC of peptide $\mathbf{3 g}$}

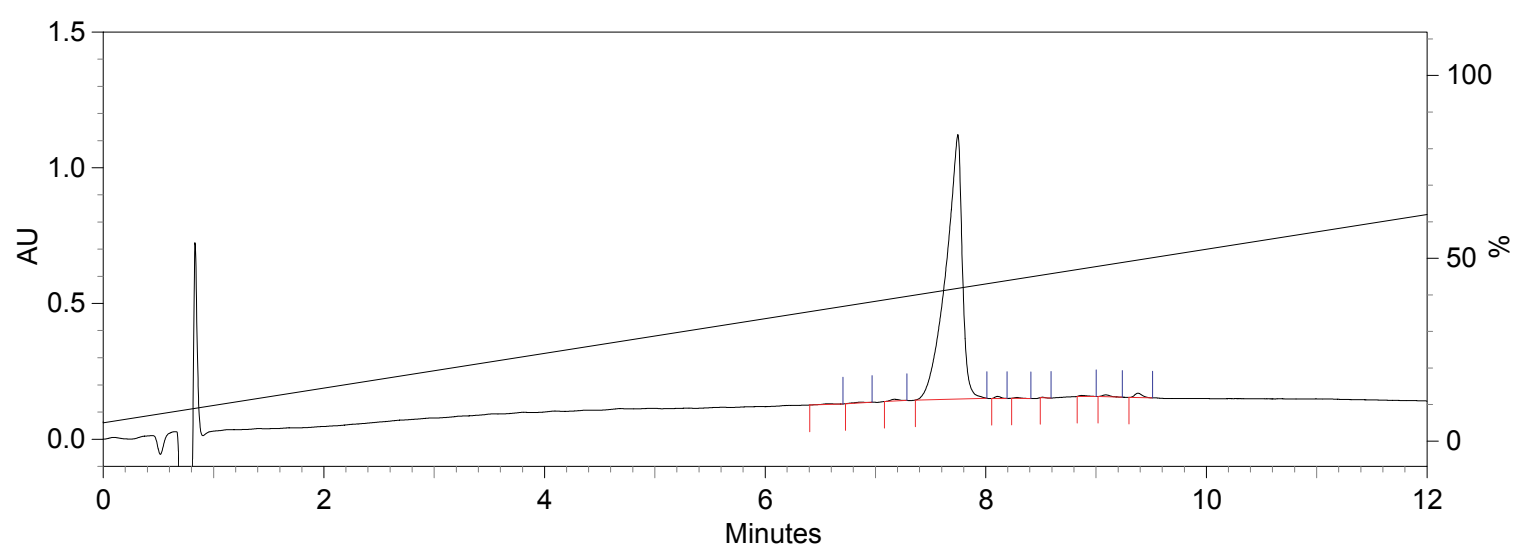

\begin{tabular}{|c|c|c|c|c|}
\hline Time & Area & Area \% & Height & Height $\%$ \\
\hline 6.050 & 7156 & 0.05 & 2184 & 0.21 \\
\hline 6.947 & 7992 & 0.06 & 1392 & 0.13 \\
\hline 7.150 & 4861 & 0.04 & 1390 & 0.13 \\
\hline 7.803 & 12620765 & 96.93 & 985636 & 93.73 \\
\hline 8.163 & 22173 & 0.17 & 3745 & 0.36 \\
\hline 8.377 & 2987 & 0.02 & 754 & 0.07 \\
\hline 8.858 & 56340 & 0.43 & 8873 & 0.84 \\
\hline 9.028 & 298184 & 2.29 & 47550 & 4.52 \\
\hline \multirow[t]{2}{*}{ Totals } & & & & \\
\hline & 13020458 & 100.00 & 1051524 & 100.00 \\
\hline
\end{tabular}

$\mathrm{C}=214 \mathrm{~nm} .80$ SB Zorbax column gradient $5 \%-100 \%$ solvent $B$ over 20 min. solvent $\mathrm{A}$ : Water (TFA $0.1 \%$ ); solvent $\mathrm{B}: \mathrm{CH}_{3} \mathrm{CN}$ (TFA $0.1 \%$ ) flow $=1 \mathrm{~mL} / \mathrm{min}$ 

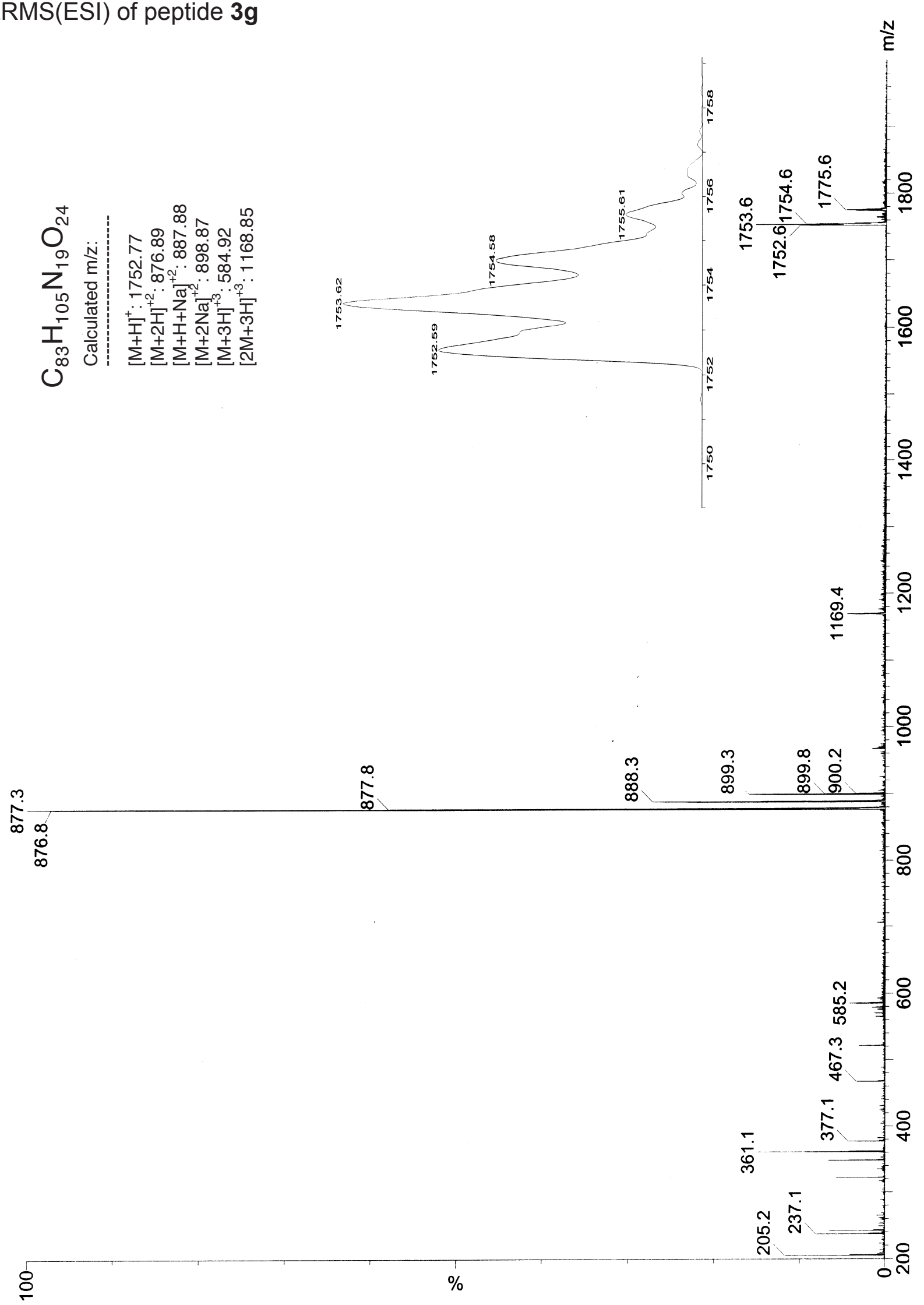

$\stackrel{\substack{\infty \\ \infty}}{\infty}$

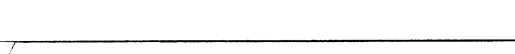

递 


\section{Analytical RP-HPLC of peptide $3 \mathrm{~h}$}

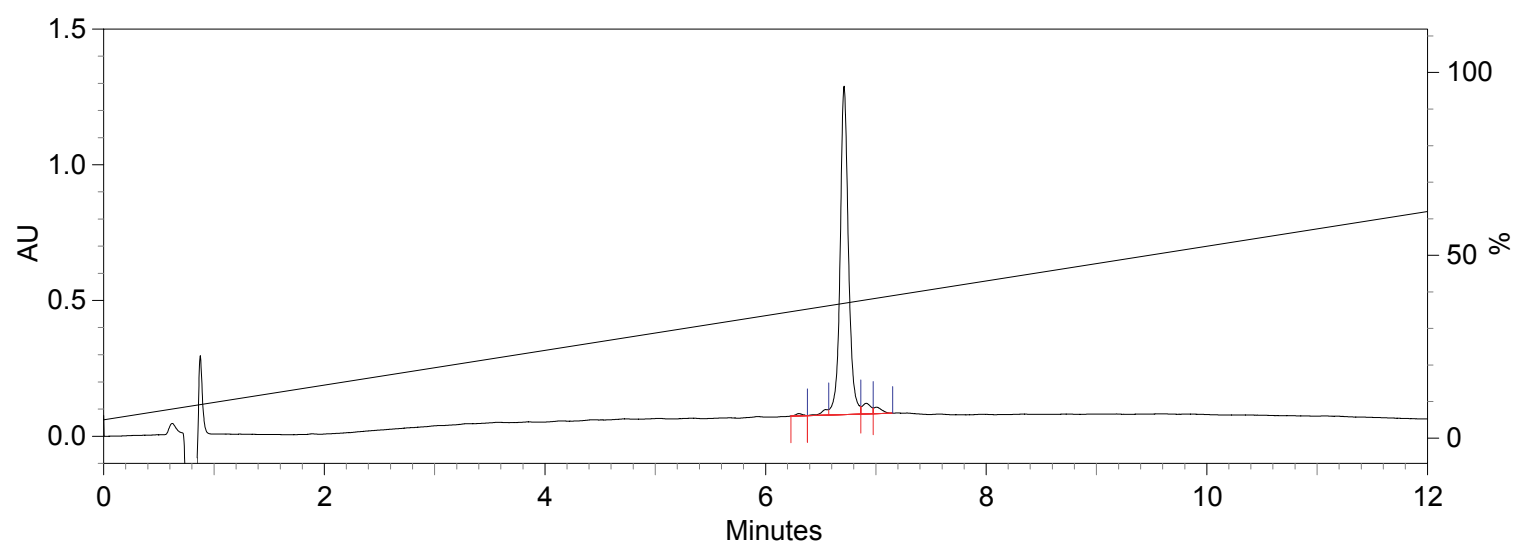

\begin{tabular}{rrrrr}
\multicolumn{1}{r}{ Time } & Area & Area \% & Height & Height \% \\
\hline 6.302 & 16701 & 0.49 & 4058 & 0.62 \\
6.543 & 45846 & 1.35 & 10079 & 1.55 \\
6.712 & 3167072 & 93.29 & 604981 & 93.01 \\
6.912 & 105520 & 3.11 & 19596 & 3.01 \\
7.003 & 59767 & 1.76 & 11765 & 1.81 \\
\hline \multicolumn{2}{|c|}{3394906} & 100.00 & 650479 & 100.00 \\
\hline
\end{tabular}

$\square=214 \mathrm{~nm} .80$ SB Zorbax column gradient $5 \%-100 \%$ solvent $B$ over 20 min. solvent $\mathrm{A}$ : Water (TFA $0.1 \%$ ); solvent $\mathrm{B}: \mathrm{CH}_{3} \mathrm{CN}$ (TFA $0.1 \%$ ) flow $=1 \mathrm{~mL} / \mathrm{min}$ 
LRMS(ESI) of peptide $\mathbf{3 h}$

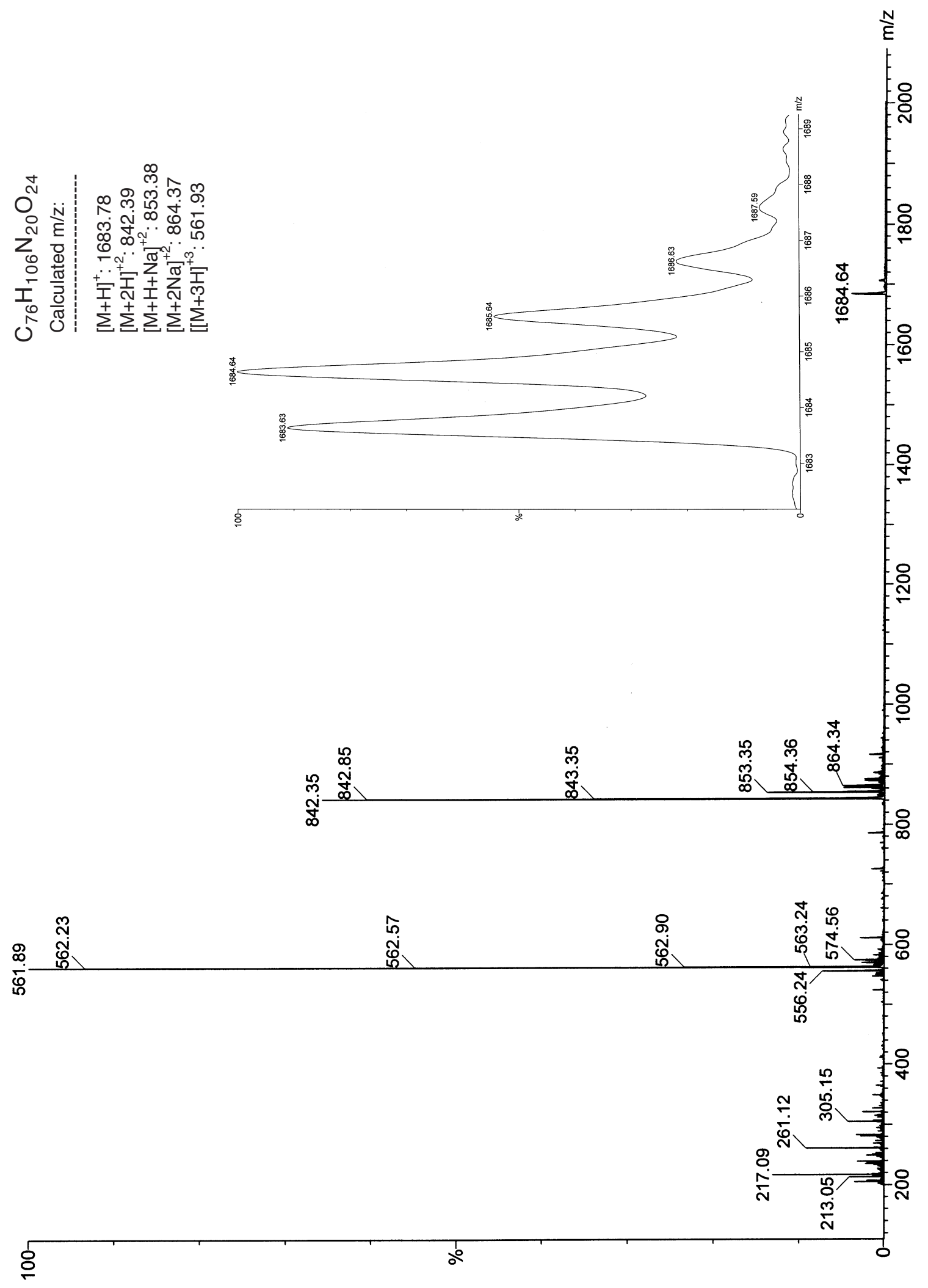




\section{Analytical RP-HPLC of peptide $\mathbf{3 i}$}

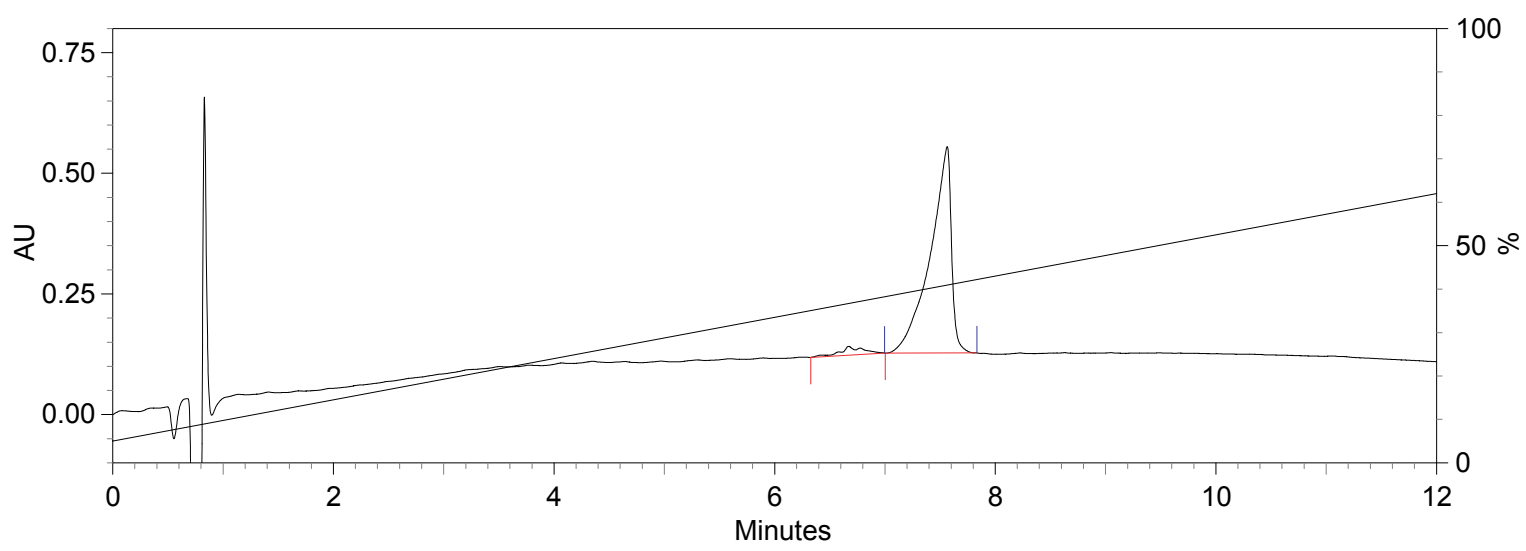

\begin{tabular}{r|rrrrr} 
Time & Area & Area \% & Height & Height \% \\
\hline 6.672 & 126268 & 4.25 & 9159 & 4.11 \\
7.565 & 2841441 & 95.75 & 213777 & 95.89 \\
\hline Totals & 2967709 & 100.00 & 222936 & 100.00 \\
\hline
\end{tabular}

$\mathrm{C}=214 \mathrm{~nm} .80$ SB Zorbax column

gradient $5 \%-100 \%$ solvent $B$ over 20 min.

solvent $\mathrm{A}$ : Water (TFA $0.1 \%$ ); solvent $\mathrm{B}: \mathrm{CH}_{3} \mathrm{CN}$ (TFA $0.1 \%$ )

flow $=1 \mathrm{~mL} / \mathrm{min}$ 


\section{LRMS(ESI) of peptide $\mathbf{3 i}$}

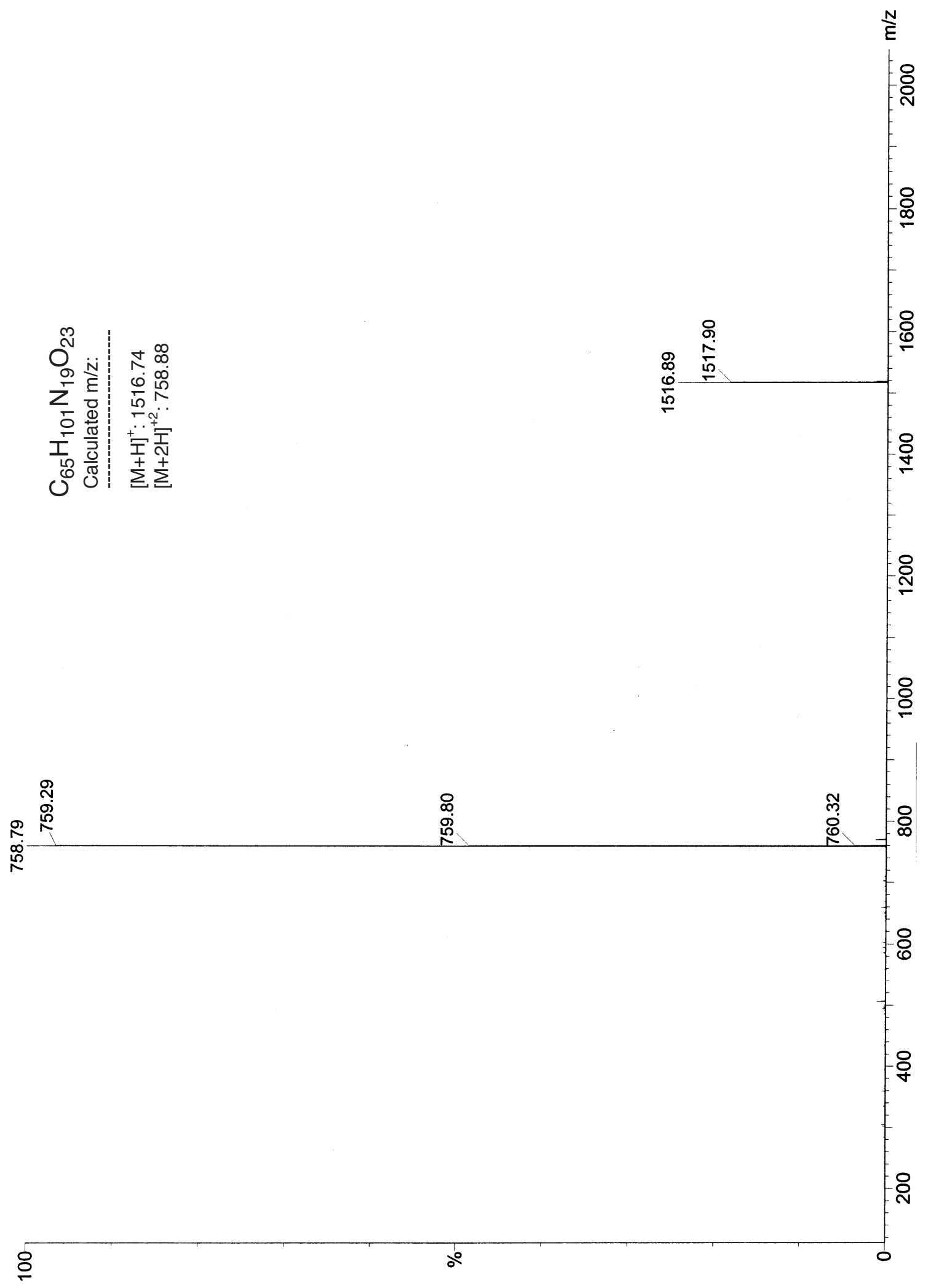




\section{Analytical RP-HPLC of peptide $\mathbf{3 j}$}

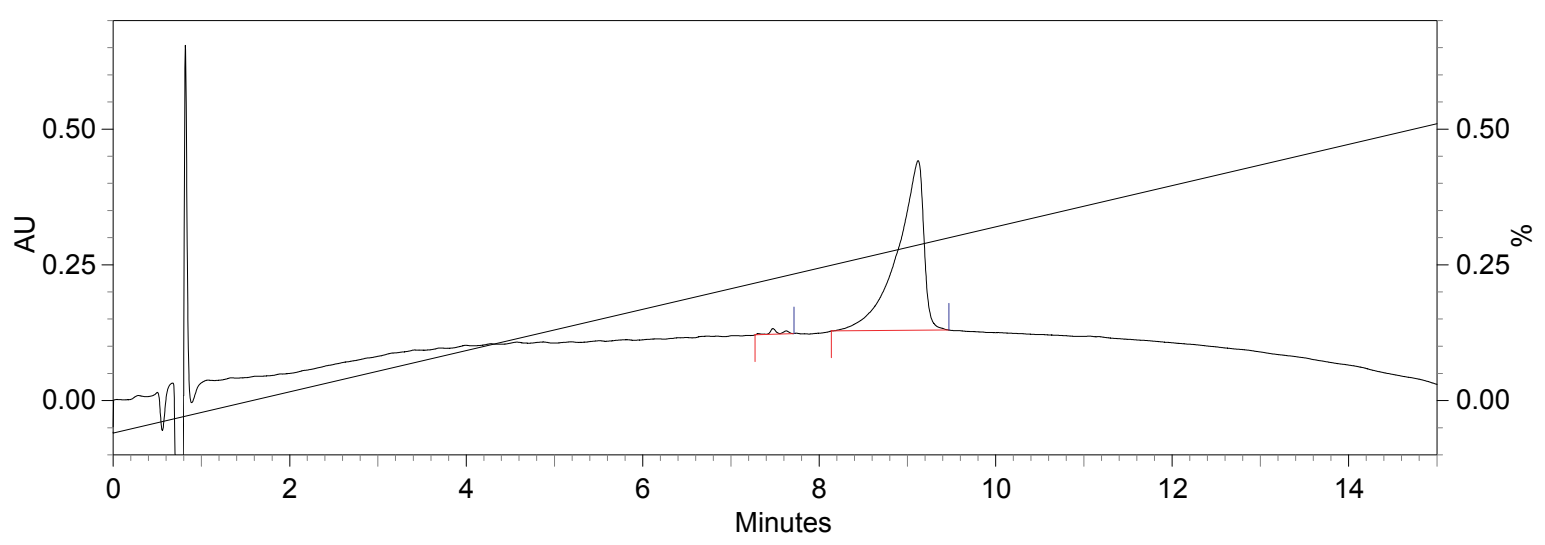

\begin{tabular}{|rrrrrr|}
\hline Time & Area & Area \% & Height & Height \% \\
\hline 7.478 & 37222 & 1.14 & 5209 & 3.22 \\
9.122 & 3231080 & 98.86 & 156315 & 96.78 \\
\hline Totals & 3268302 & 100.00 & 161524 & 100.00 \\
\hline
\end{tabular}

$\square=214 \mathrm{~nm} .80 \mathrm{SB}$ Zorbax column gradient $5 \%-100 \%$ solvent $B$ over 20 min. solvent A: Water (TFA 0.1\%); solvent $\mathrm{B}: \mathrm{CH}_{3} \mathrm{CN}$ (TFA $0.1 \%$ ) flow $=1 \mathrm{~mL} / \mathrm{min}$ 


\section{LRMS(ESI) of peptide $\mathbf{3 j}$}
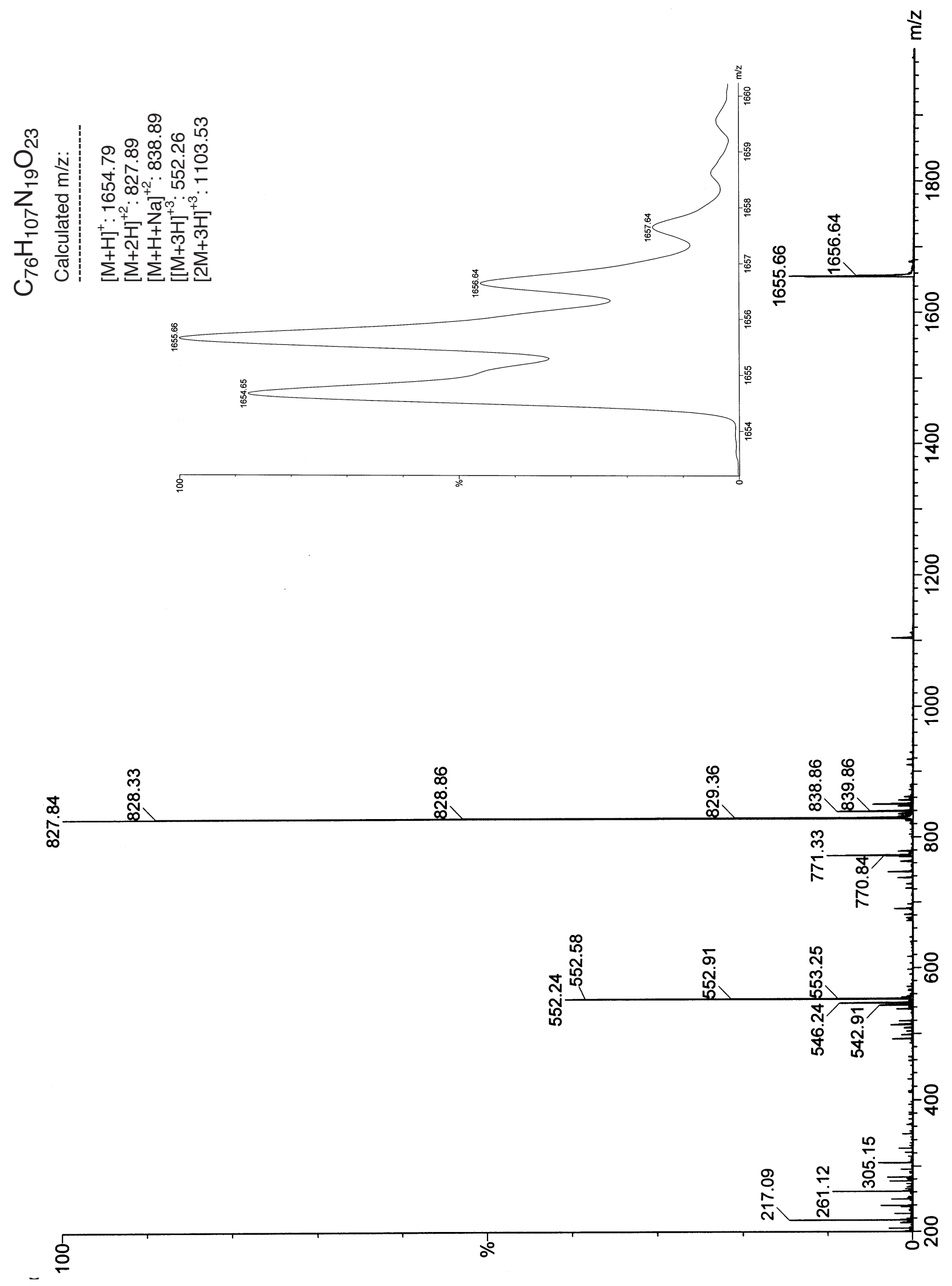


\section{Analytical RP-HPLC of peptide $\mathbf{3 k}$}

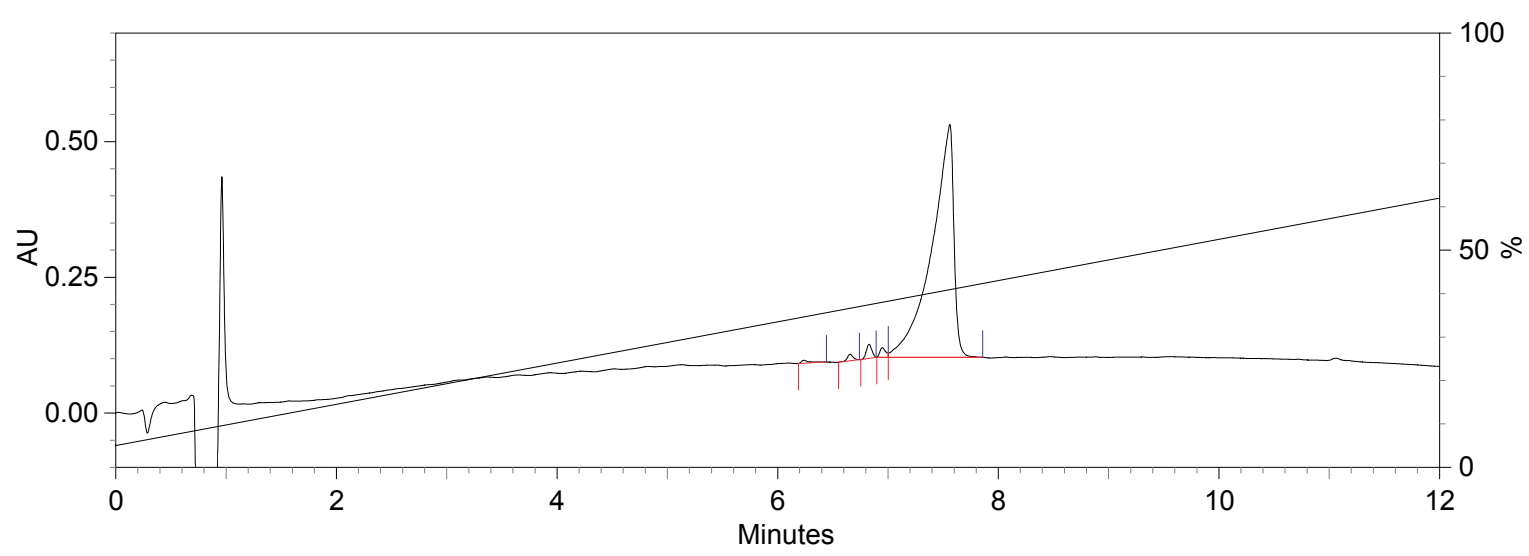

\begin{tabular}{|c|c|c|c|c|}
\hline Time & Area & Area \% & Height & Height $\%$ \\
\hline 6.235 & 14137 & 0.46 & 2790 & 1.14 \\
\hline 6.657 & 22037 & 0.72 & 6144 & 2.50 \\
\hline 6.828 & 45007 & 1.47 & 13044 & 5.32 \\
\hline 6.950 & 32884 & 1.07 & 8887 & 3.62 \\
\hline 7.560 & 2954209 & 96.28 & 214479 & 87.42 \\
\hline Totals & 3068274 & 100.00 & 245344 & 100.00 \\
\hline
\end{tabular}

$\square=214 \mathrm{~nm} .80$ SB Zorbax column gradient $5 \%-100 \%$ solvent $B$ over 20 min. solvent $\mathrm{A}$ : Water (TFA $0.1 \%$ ); solvent $\mathrm{B}: \mathrm{CH}_{3} \mathrm{CN}$ (TFA $0.1 \%$ ) flow $=1 \mathrm{~mL} / \mathrm{min}$ 


\section{LRMS(ESI) of peptide $\mathbf{3 k}$}

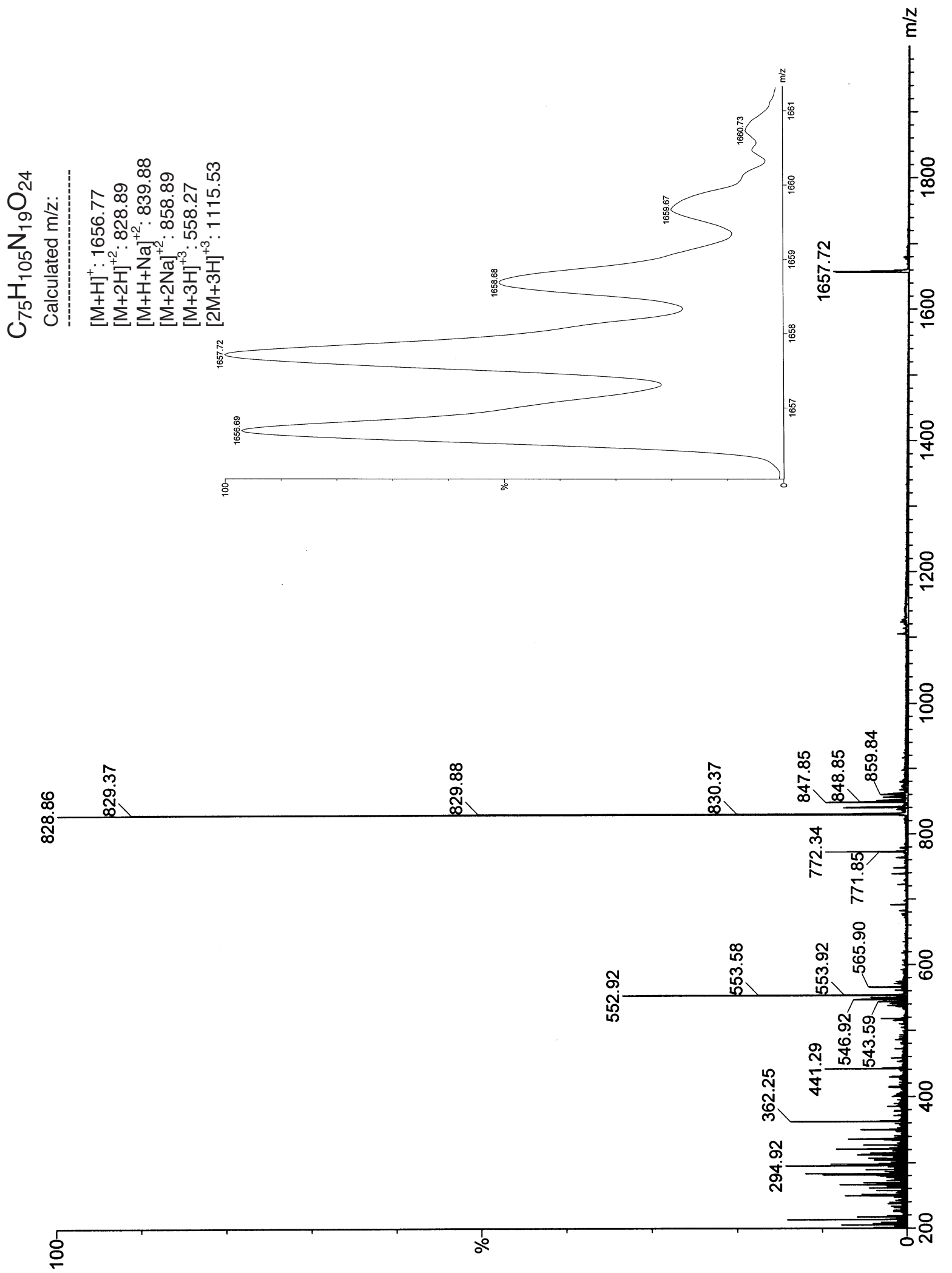


Analytical RP-HPLC of peptide $\mathbf{3 l}$

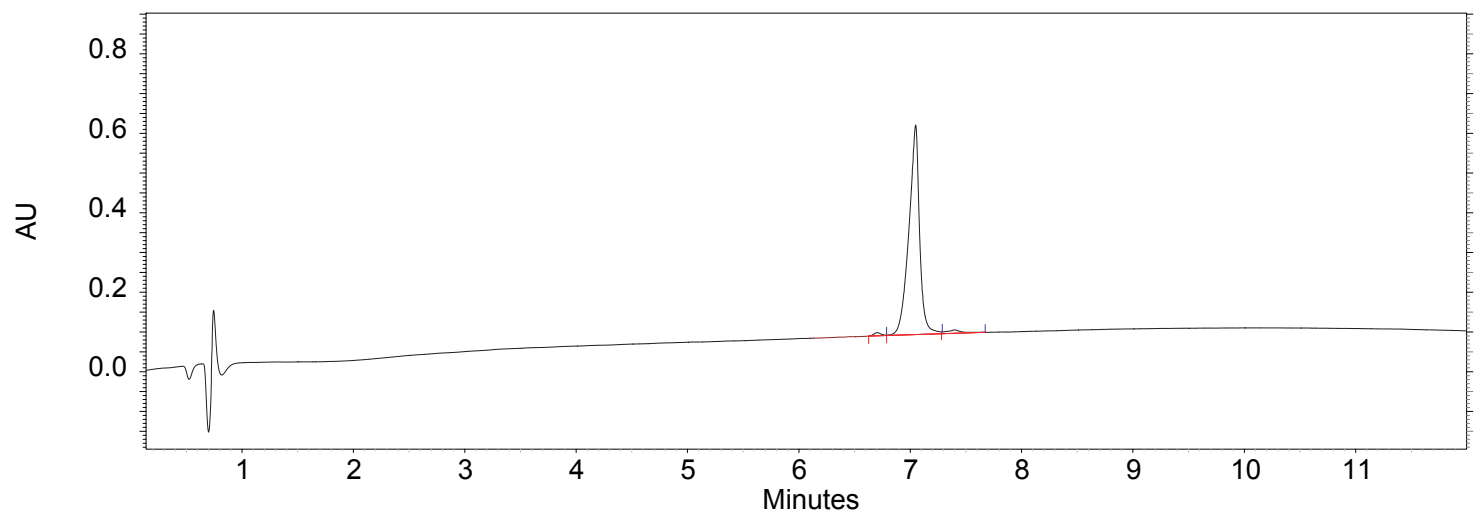

\begin{tabular}{rrrrr} 
Time & Area & Area \% & Height & Height \% \\
\hline 6.705 & 35276 & 0.97 & 7971 & 1.47 \\
7.048 & 3526288 & 96.90 & 527362 & 96.98 \\
7.397 & 77577 & 2.13 & 8428 & 1.55 \\
\hline \multicolumn{7}{|r|}{} & & & \\
\hline Totals & 3639141 & 100.00 & 543761 & 100.00 \\
\hline
\end{tabular}

$\square=214 \mathrm{~nm} .80$ SB Zorbax column

gradient $5 \%-100 \%$ solvent $B$ over 20 min.

solvent $\mathrm{A}$ : Water (TFA $0.1 \%$ ); solvent $\mathrm{B}: \mathrm{CH}_{3} \mathrm{CN}$ (TFA $0.1 \%$ )

flow $=1 \mathrm{~mL} / \mathrm{min}$ 


\section{LRMS(ESI) of peptide $\mathbf{3 I}$}

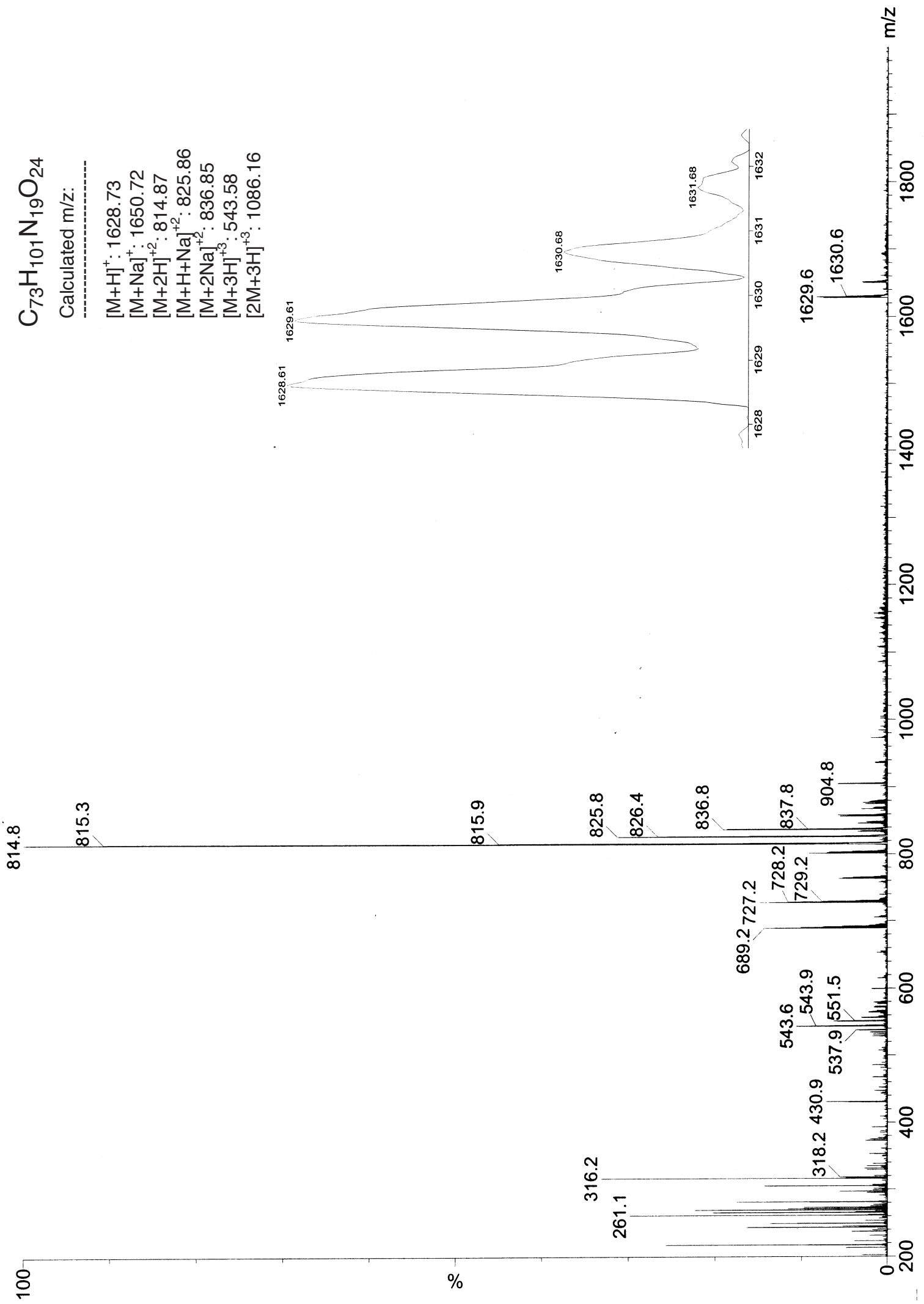




\section{Analytical RP-HPLC of peptide $3 \mathrm{~m}$}

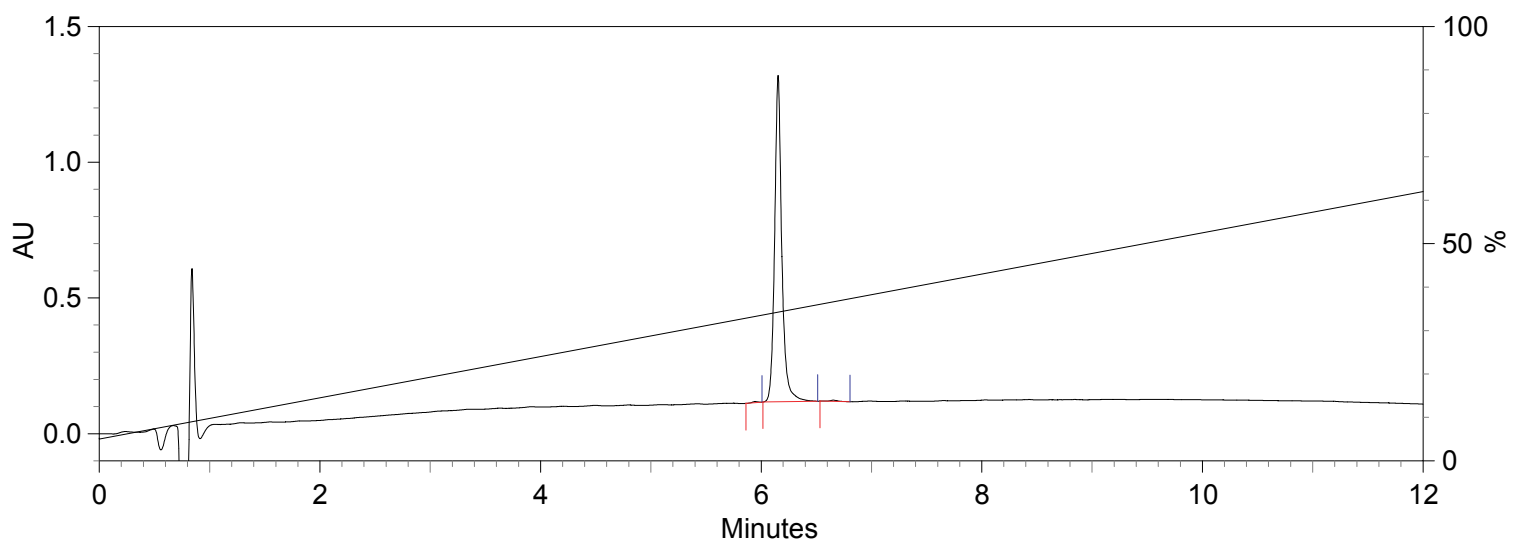

\begin{tabular}{rrrrrr}
\multicolumn{1}{r}{ Time } & Area & Area \% & Height & Height \% \\
\hline 5.945 & 6298 & 0.23 & 1929 & 0.32 \\
6.153 & 2721128 & 99.34 & 601362 & 99.28 \\
6.657 & 11726 & 0.43 & 2423 & 0.40 \\
\hline \multicolumn{2}{|c|}{ Totals } & 2739152 & 100.00 & 605714 & 100.00 \\
\hline
\end{tabular}

C= $214 \mathrm{~nm} .80$ SB Zorbax column gradient $5 \%-100 \%$ solvent $B$ over 20 min. solvent $\mathrm{A}$ : Water (TFA $0.1 \%$ ); solvent $\mathrm{B}$ : $\mathrm{CH}_{3} \mathrm{CN}$ (TFA $0.1 \%$ ) flow $=1 \mathrm{~mL} / \mathrm{min}$ 


\section{LRMS(ESI) of peptide $3 \mathrm{~m}$}

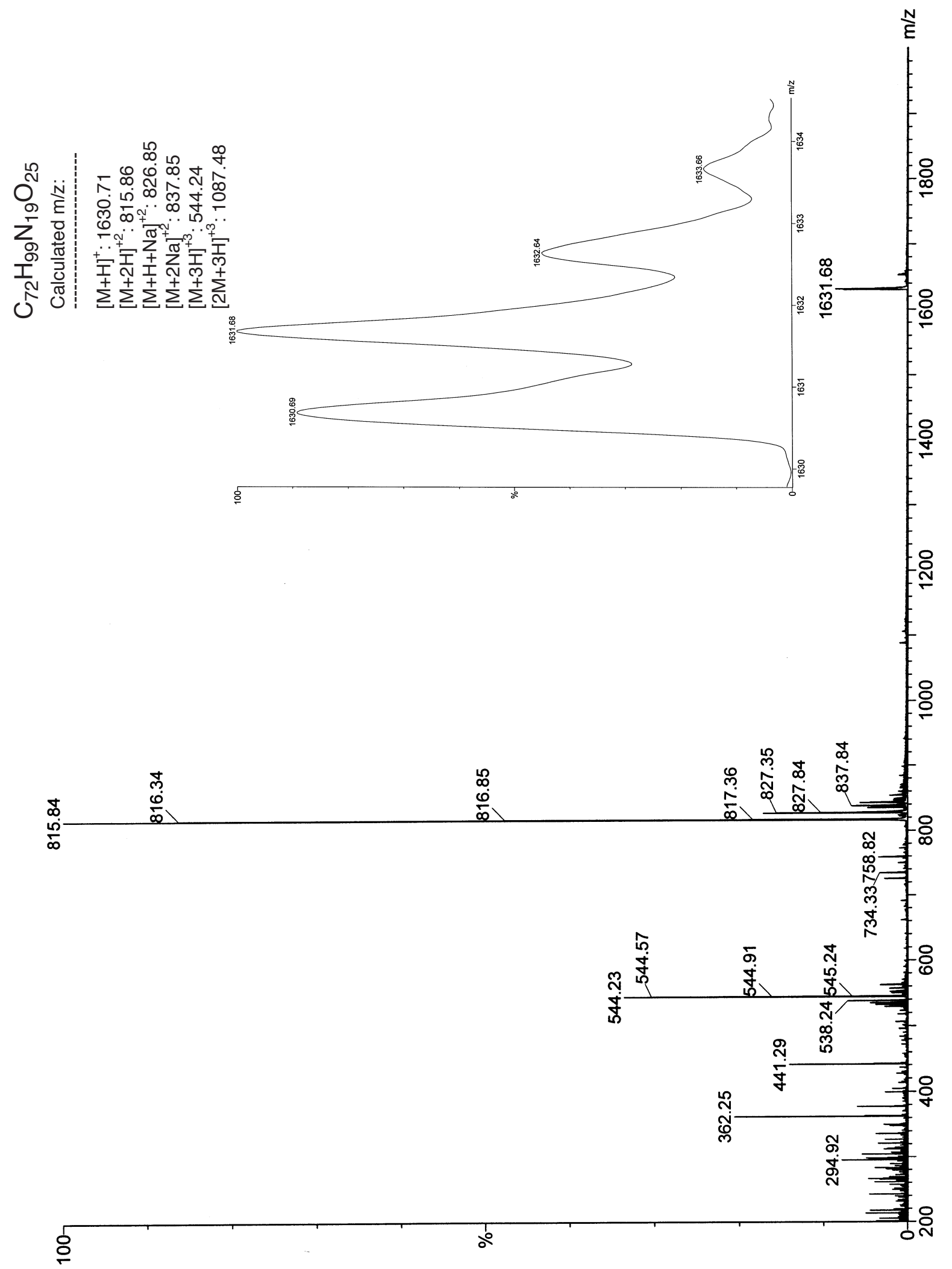




\section{Analytical RP-HPLC of peptide $3 n$}

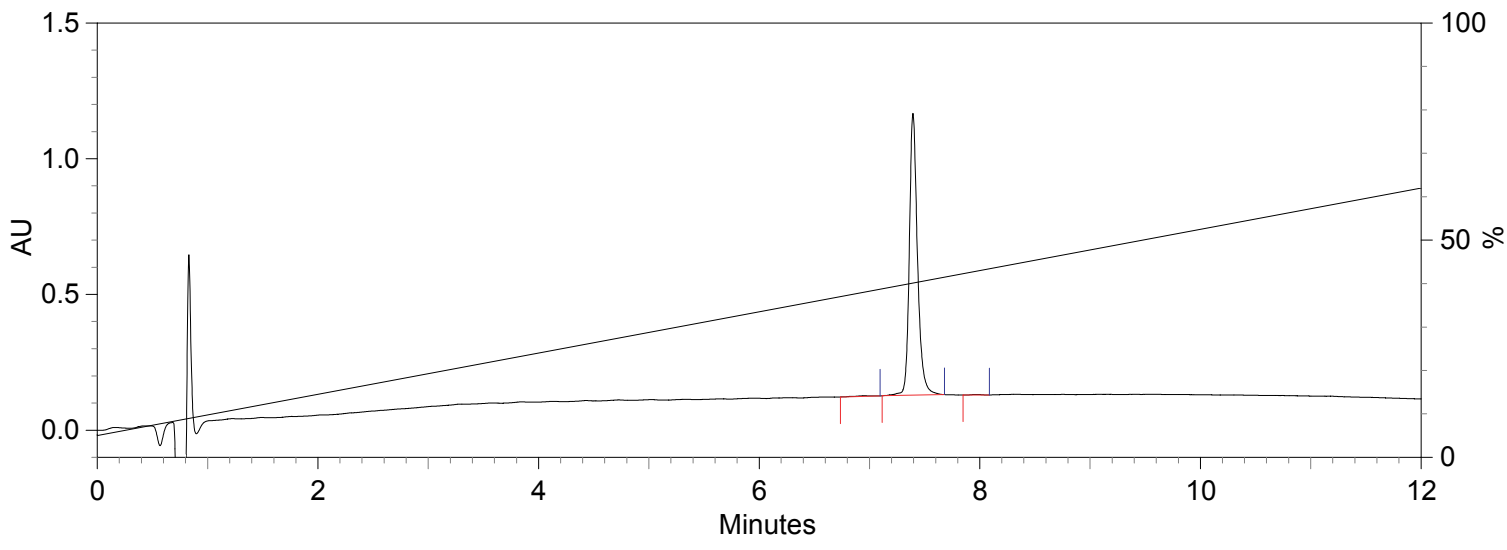

\begin{tabular}{rrrrrr}
\multicolumn{1}{r}{ Time } & Area & Area \% & Height & Height \% \\
\hline 6.950 & 9426 & 0.35 & 1048 & 0.20 \\
7.393 & 2680325 & 99.46 & 519324 & 99.62 \\
7.967 & 5215 & 0.19 & 918 & 0.18 \\
\hline \multicolumn{2}{|c|}{ Totals } & 2694966 & 100.00 & 521290 & 100.00 \\
\hline
\end{tabular}

$\square=214 \mathrm{~nm} .80$ SB Zorbax column gradient $5 \%-100 \%$ solvent $B$ over 20 min. solvent $\mathrm{A}$ : Water (TFA $0.1 \%$ ); solvent $\mathrm{B}: \mathrm{CH}_{3} \mathrm{CN}$ (TFA $0.1 \%$ ) flow $=1 \mathrm{~mL} / \mathrm{min}$ 
LRMS(ESI) of peptide $3 n$

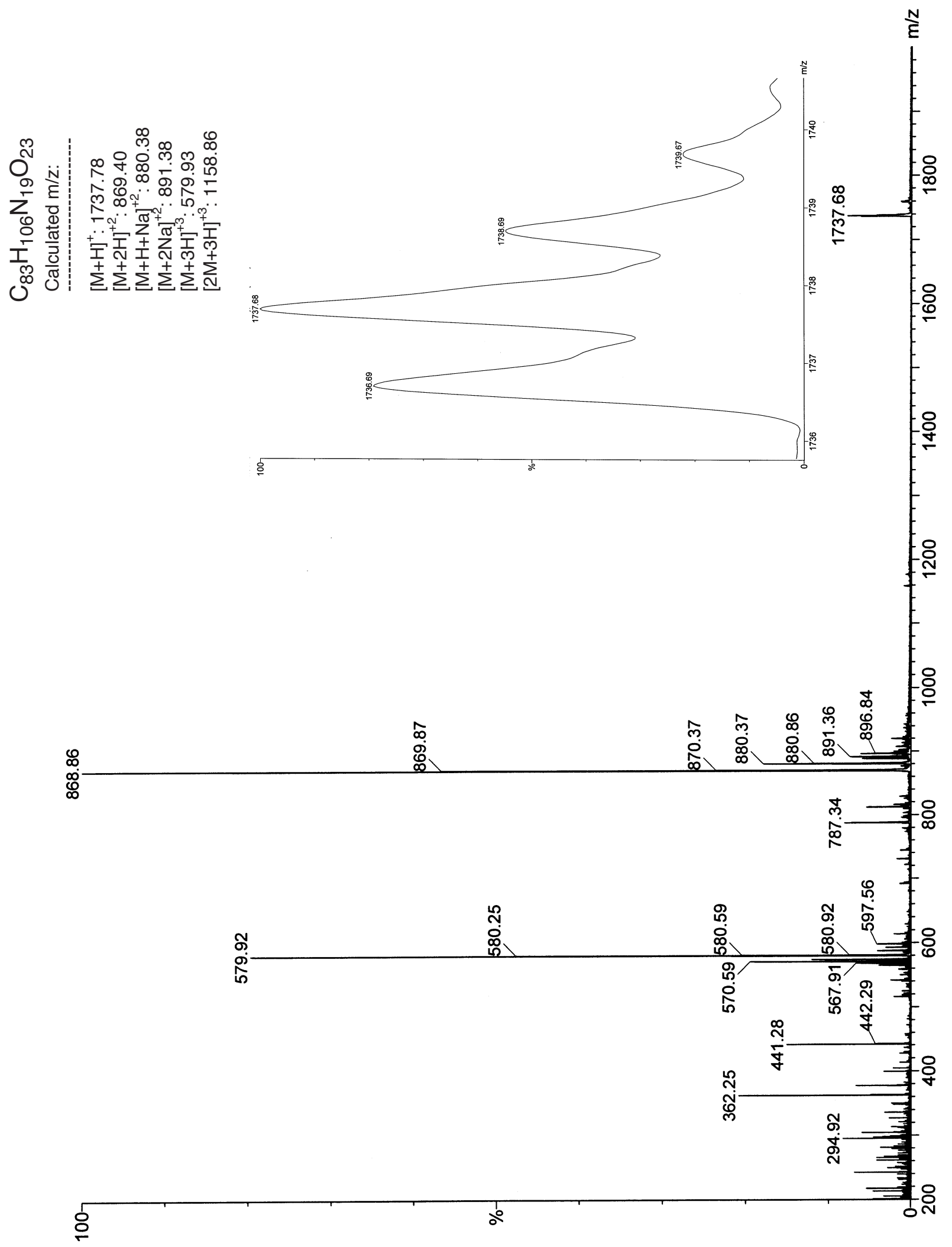




\section{Analytical RP-HPLC of peptide 6}

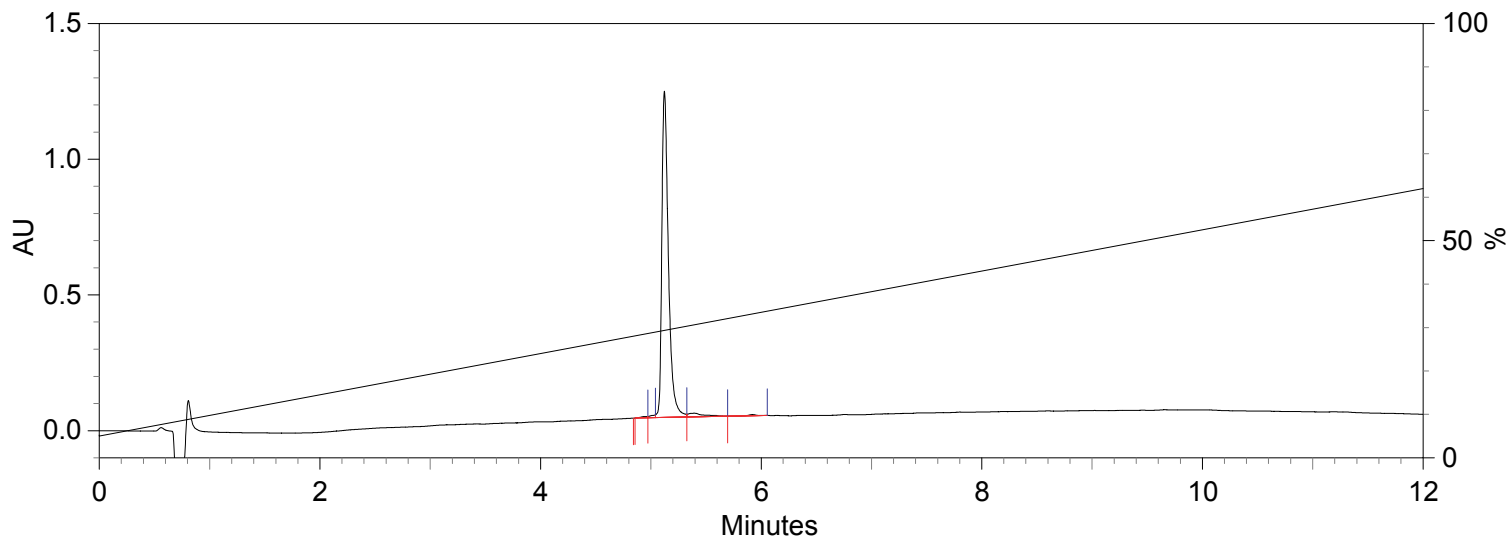

\begin{tabular}{rrrrr} 
Time & Area & Area \% & Height & Height \% \\
\hline 4.858 & 10107 & 0.00 & 0 & 0.00 \\
5.040 & 15629 & 0.00 & 5037 & 0.82 \\
5.123 & 409691949019 & 100.00 & 601055 & 97.69 \\
5.392 & 71540 & 0.00 & 6941 & 1.13 \\
5.913 & 10014 & 0.00 & 2208 & 0.36 \\
\hline \multicolumn{5}{|c|}{} \\
\hline Totals & 409692056309 & 100.00 & 615241 & 100.00 \\
\hline
\end{tabular}

$\square=214 \mathrm{~nm} .80$ SB Zorbax column gradient $5 \%-100 \%$ solvent $B$ over 20 min. solvent $\mathrm{A}$ : Water (TFA $0.1 \%$ ); solvent $\mathrm{B}: \mathrm{CH}_{3} \mathrm{CN}$ (TFA $0.1 \%$ ) flow $=1 \mathrm{~mL} / \mathrm{min}$ 
LRMS(ESI) of peptide 6
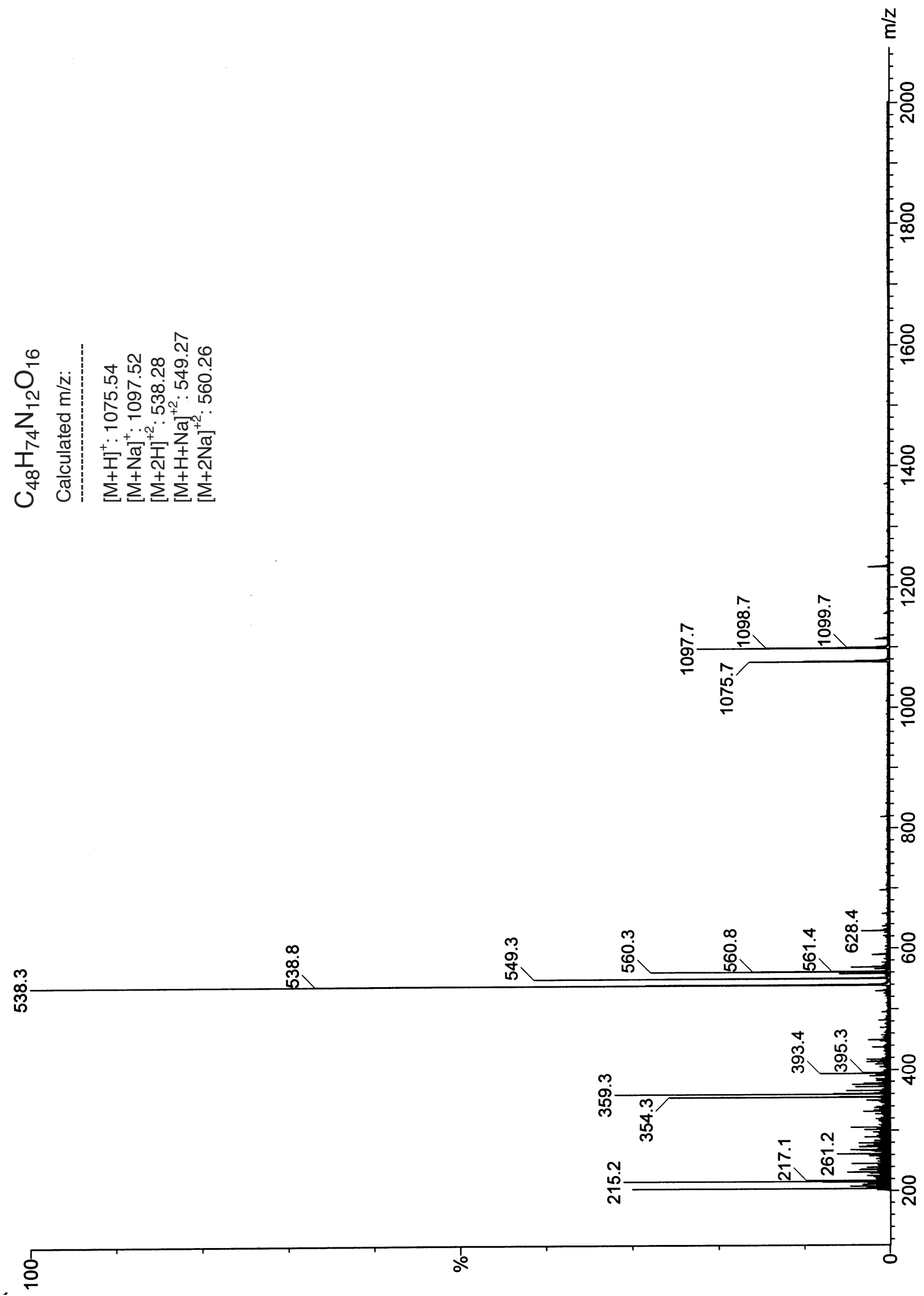


\section{${ }^{1} \mathrm{H}$ NMR characterization of peptide $3 \mathrm{a}$}

All solutions were prepared gravimetrically by dissolving an appropriate weight of the peptides in an appropriate volume of solvent. In calculating molecular weights, all amino groups were assumed to be protonated as TFA salts.

${ }^{1} \mathrm{H}$ NMR assignments of the peptide 3a oligomer. The ${ }^{1} \mathrm{H}$ NMR resonances of the oligomer were assigned by $800 \mathrm{MHz}$ TOCSY and NOESY experiments at $280 \mathrm{~K}$ and a $500 \mathrm{MHz}$ Tr-ROESY ${ }^{10}$ experiment at $278 \mathrm{~K}$, using water presaturation or WATERGATE as needed, on 8.5 $\mathrm{mM}$ solutions of peptide 3a in $\mathrm{D}_{2} \mathrm{O}$ and $\mathrm{H}_{2} \mathrm{O}-\mathrm{D}_{2} \mathrm{O}$ (90:10). The $500 \mathrm{MHz}$ Tr-ROESY and 800 $\mathrm{MHz}$ TOCSY spectra were acquired using 300-ms and 50-ms spin-lock mixing times, respectively. The $800 \mathrm{MHz}$ NOESY experiments were carried out with a 75-ms mixing time. All $800 \mathrm{MHz} 2 \mathrm{D}$ spectra were acquired with 4096 data points in the $f_{2}$ domain and 512 data points in

the $f_{l}$ domain and were processed by zero-filling to a final matrix of $2048 \times 2048$ real points. The $500 \mathrm{MHz}$ Tr-ROESY spectrum was acquired with 2048 data points in the $f_{2}$ domain and 512 data points in the $f_{l}$ domain and was processed by zero-filling to a final matrix of $1024 \times 1024$ real points.

${ }^{1} \mathrm{H}$ NMR assignments of the peptide 3a monomer. The ${ }^{1} \mathrm{H}$ NMR resonances of the monomer were assigned by $800 \mathrm{MHz}$ TOCSY and NOESY experiments at $280 \mathrm{~K}$, using water presaturation or WATERGATE as needed, on $1.0 \mathrm{mM} \mathrm{D}_{2} \mathrm{O}$ and $0.6 \mathrm{mM} \mathrm{H}_{2} \mathrm{O}-\mathrm{D}_{2} \mathrm{O}$ (90:10) solutions of peptide 3a. A 400-ms mixing time was used for $800 \mathrm{MHz}$ NOESY experiments, and a spin-lock mixing time of $50 \mathrm{~ms}$ was used for $800 \mathrm{MHz}$ TOCSY experiments. All $800 \mathrm{MHz} 2 \mathrm{D}$ spectra were acquired with 4096 data points in the $f_{2}$ domain and 512 data points in the $f_{1}$ domain and were processed by zero-filling to a final matrix of 2048 x 2048 real points.

${ }^{1} \mathbf{H}$ EXSY studies of peptide 3a. The $800 \mathrm{MHz}$ EXSY study was performed at $280 \mathrm{~K}$ on an $8.5 \mathrm{mM} \mathrm{D}_{2} \mathrm{O}-\mathrm{H}_{2} \mathrm{O}$ (10:90) solution of peptide 3a. The EXSY spectrum was acquired using the Tr-ROESY pulse sequence, ${ }^{10}$ a 50 -ms spin-lock mixing time, and WATERGATE for water suppression. The data were recorded with 4096 data points in the $f_{2}$ domain and 512 data points in the $f_{l}$ domain and processed by zero-filling to a matrix of $2048 \times 2048$ real points. The EXSY cross-peaks have the same phase as the diagonal peaks, while the ROESY cross-peaks have the opposite phase.

10 (a) Hwang, T. -L.; Shaka, A. J. J. Am. Chem Soc. 1992, 114, 3157-3159. (b) Hwang, T. -L.; Shaka, A. J. J. Magn. Reson., Ser. B 1993, 102, 155-165. 

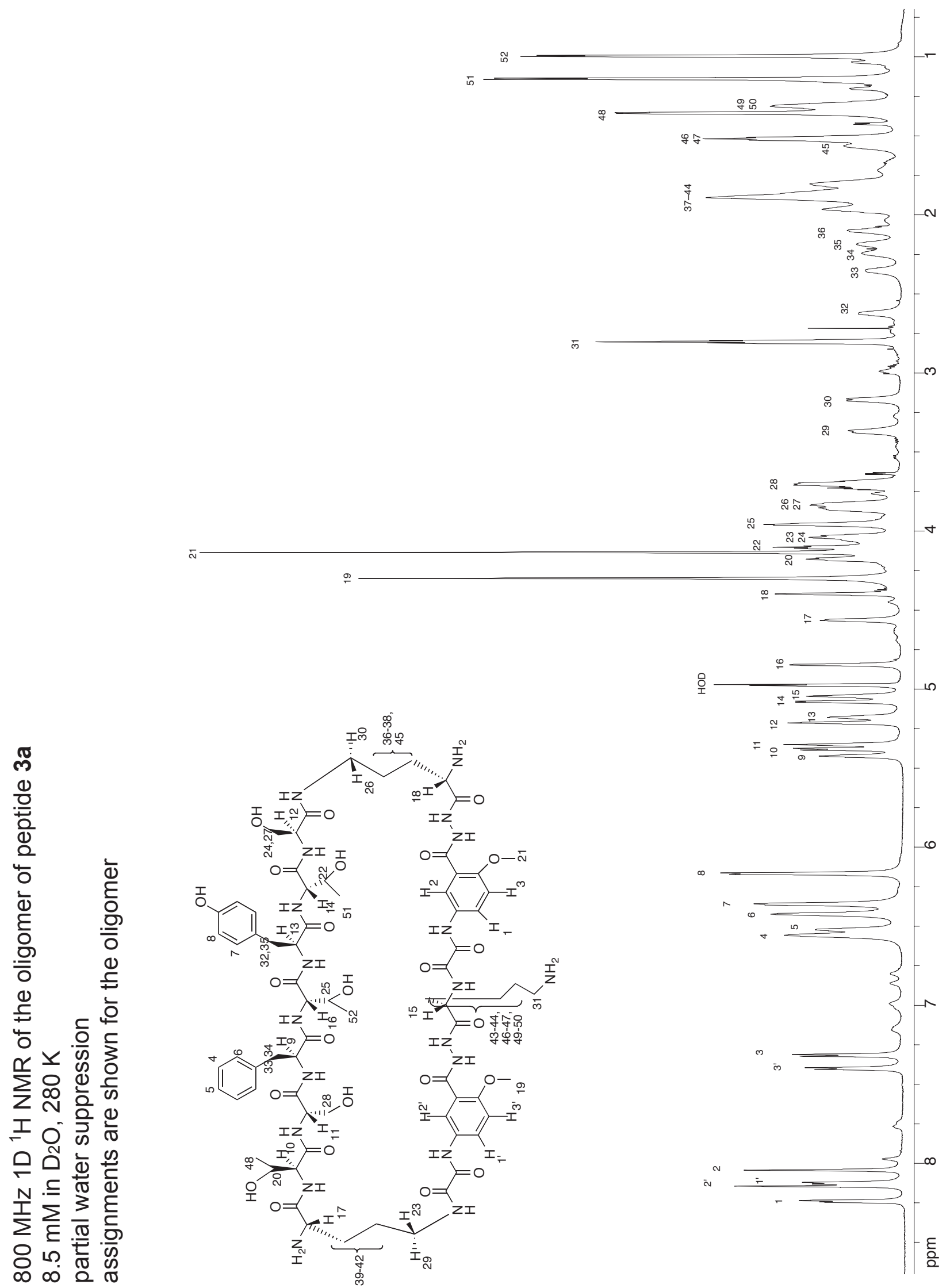
$800 \mathrm{MHz} 1 \mathrm{D}{ }^{1} \mathrm{H}$ NMR of the oligomer of peptide $\mathbf{3 a}$

$8.5 \mathrm{mM}$ in $\mathrm{D}_{2} \mathrm{O}, 280 \mathrm{~K}$

partial water suppression

assignments are shown for the oligomer
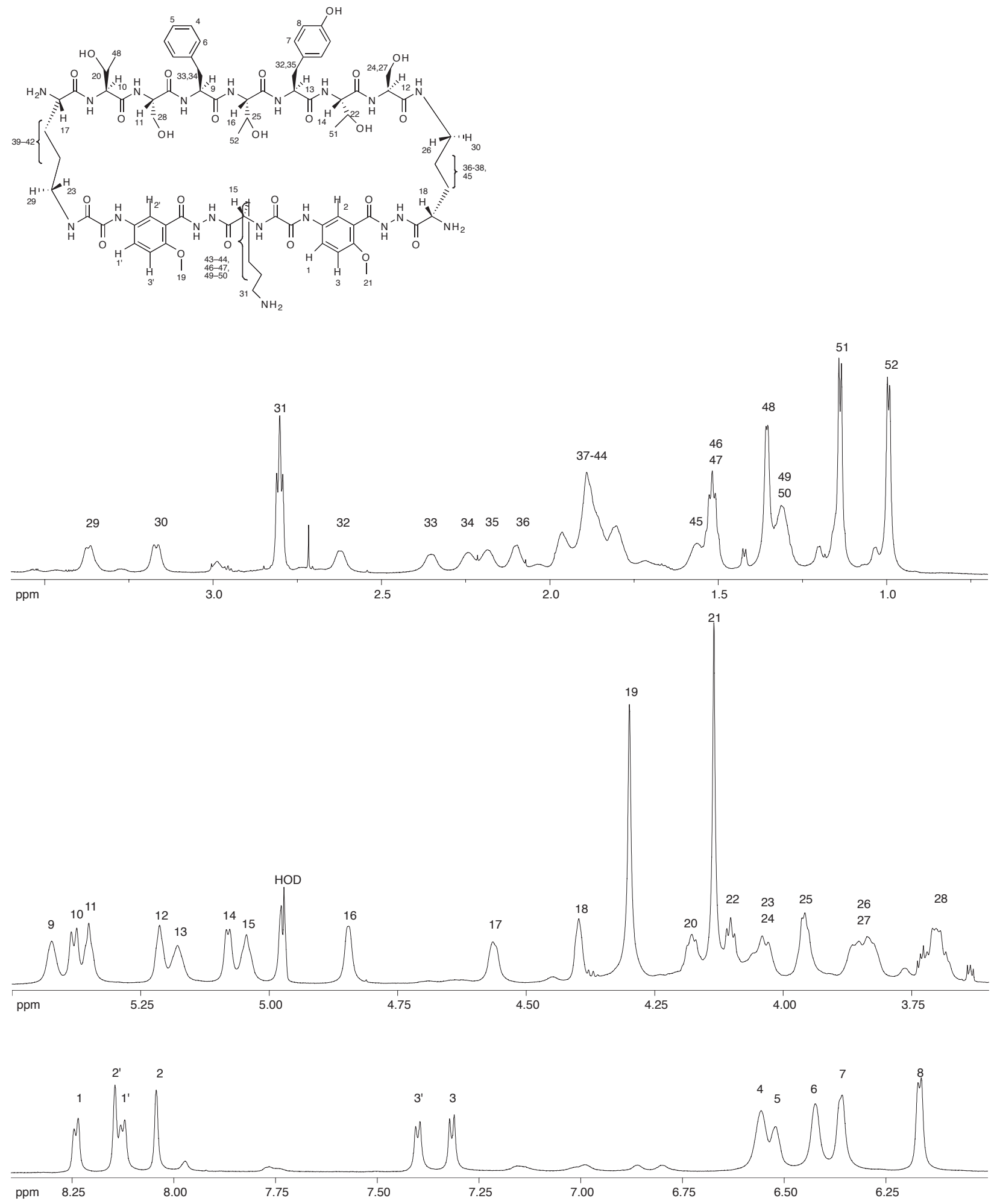
$800 \mathrm{MHz}$ TOCSY spectrum of the oligomer of peptide $3 \mathrm{a}$ $8.5 \mathrm{mM}$ in $\mathrm{D}_{2} \mathrm{O}, 280 \mathrm{~K}$ 50-ms spin-lock mixing time assignments are shown for the oligomer

$$
\text { oligomer of peptide } 3 \mathbf{a}
$$

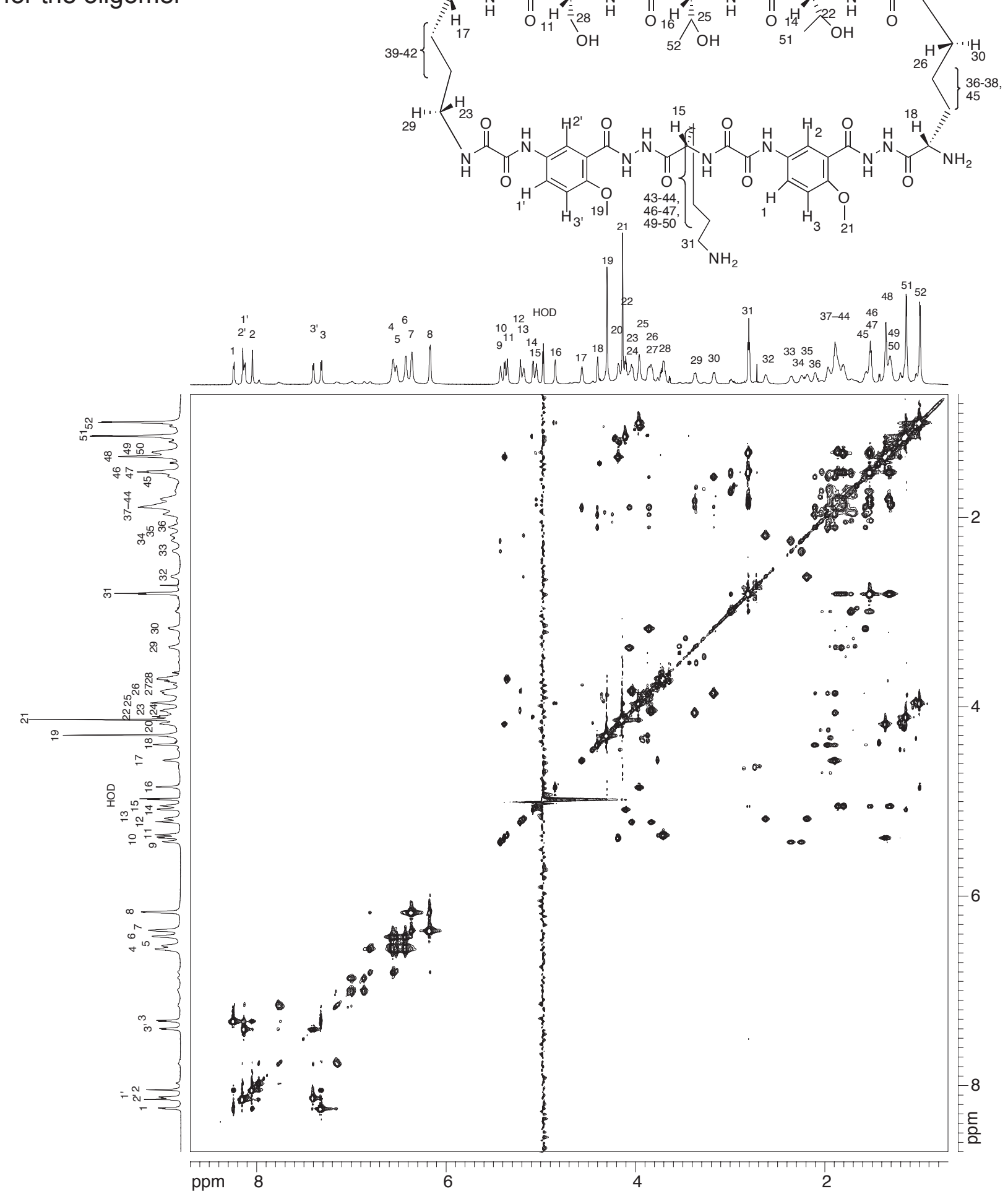


$800 \mathrm{MHz}$ TOCSY spectrum of the oligomer of peptide $3 \mathbf{a}$ $8.5 \mathrm{mM}$ in $\mathrm{D}_{2} \mathrm{O}, 280 \mathrm{~K}$ 50-ms spin-lock mixing time assignments are shown for the oligomer
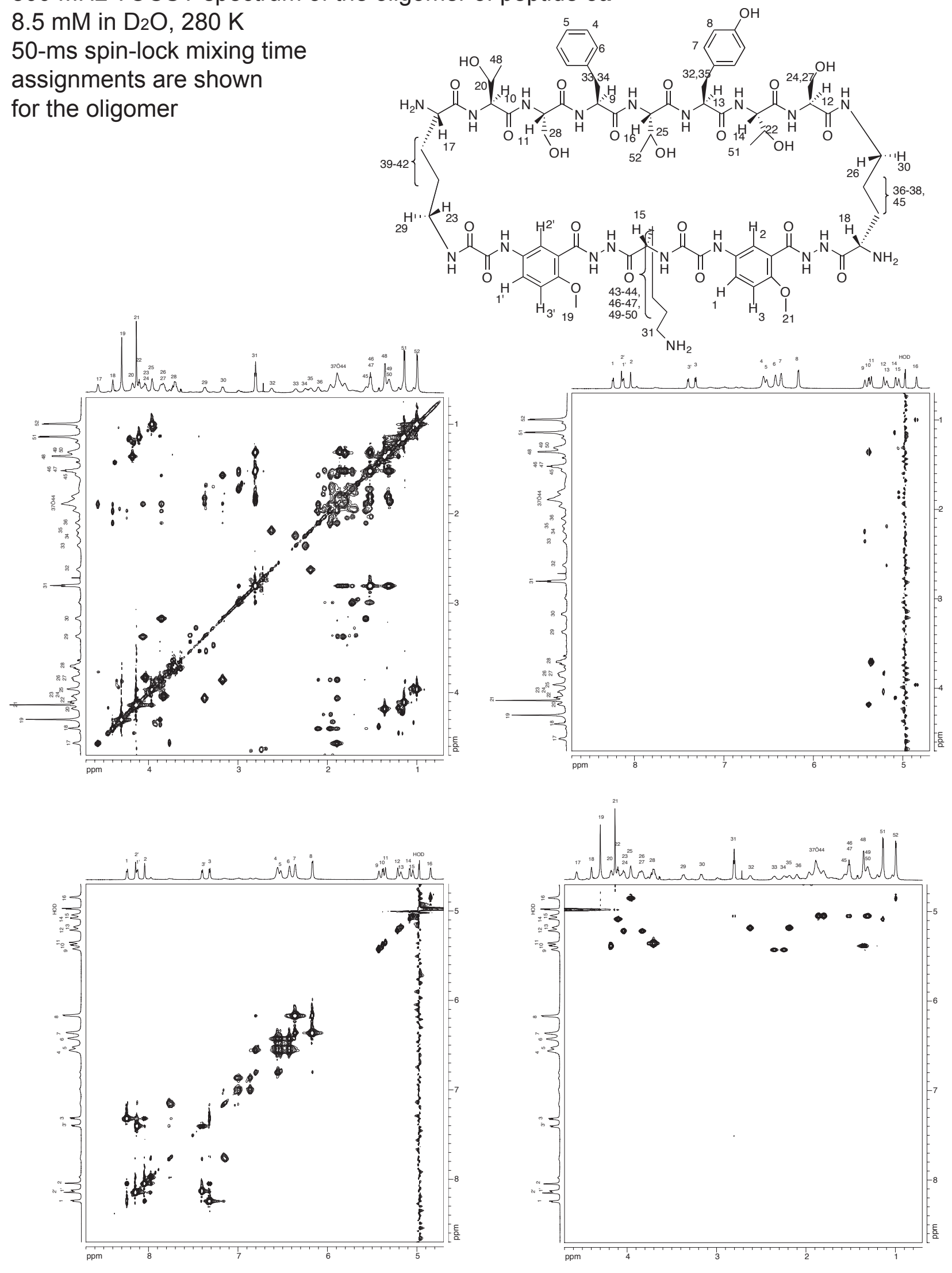
$800 \mathrm{MHz}$ NOESY spectrum of the oligomer of peptide $3 \mathrm{a}$

$8.5 \mathrm{mM}$ in $\mathrm{D}_{2} \mathrm{O}, 280 \mathrm{~K}$

75-ms mixing time

assignments are shown

for the oligomer
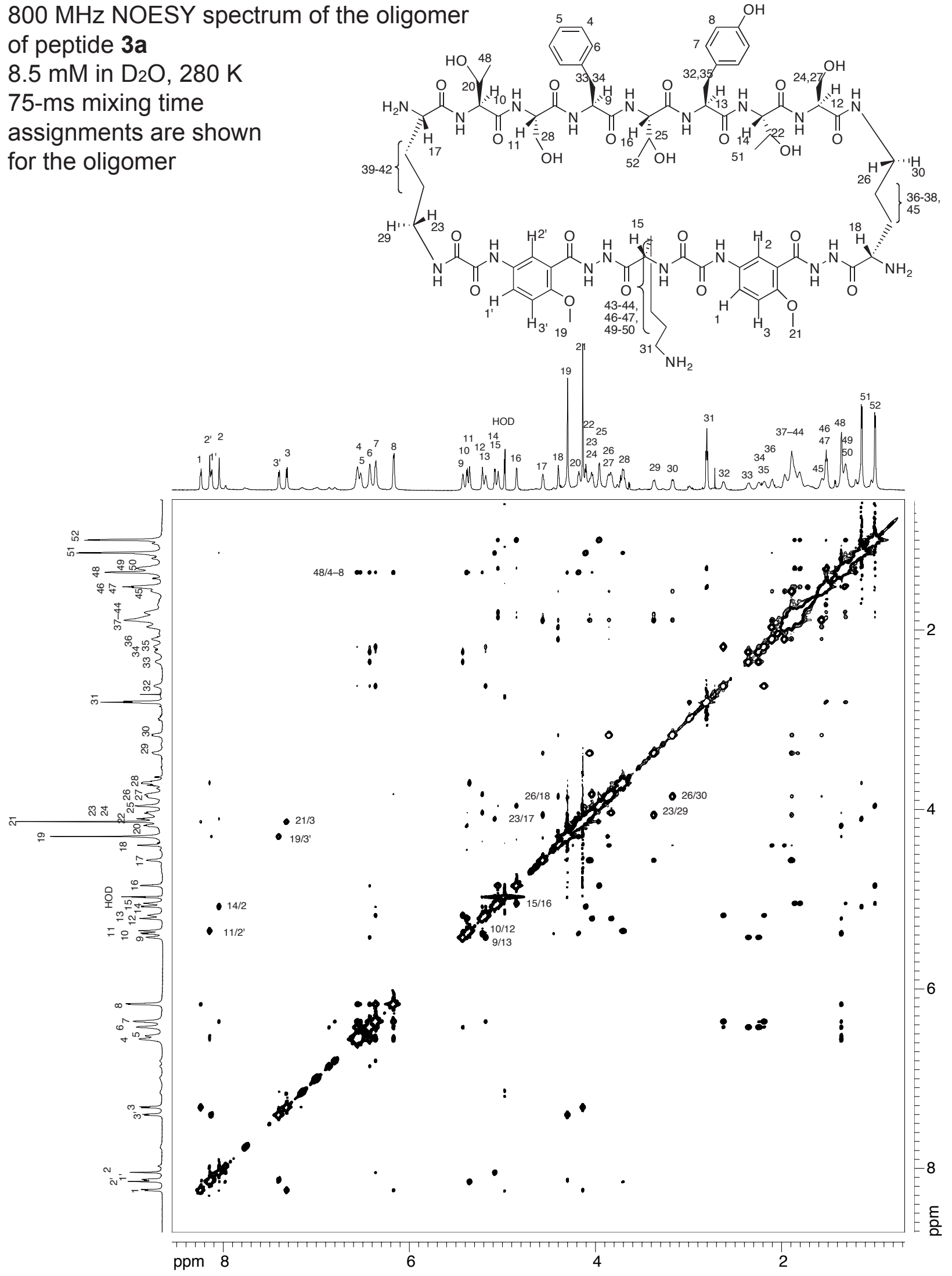
$800 \mathrm{MHz}$ NOESY spectrum of the oligomer of peptide $\mathbf{3 a}$

$8.5 \mathrm{mM}$ in $\mathrm{D}_{2} \mathrm{O}, 280 \mathrm{~K}$ 75-ms mixing time assignments are shown for the oligomer

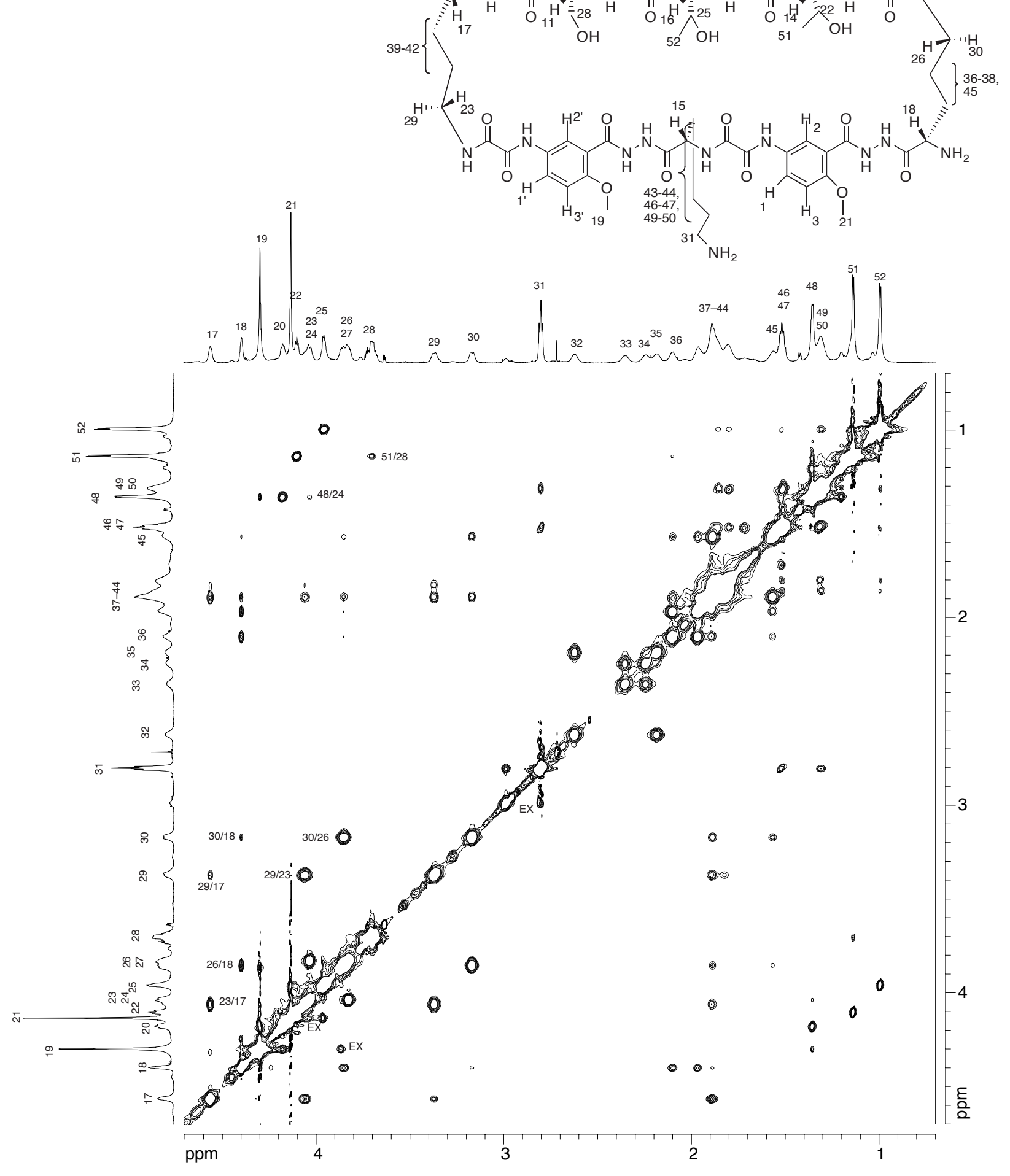


$800 \mathrm{MHz}$ NOESY spectrum of the oligomer of peptide $\mathbf{3 a}$

\section{$8.5 \mathrm{mM}$ in $\mathrm{D}_{2} \mathrm{O}, 280 \mathrm{~K}$}

75-ms mixing time

assignments are shown

for the oligomer
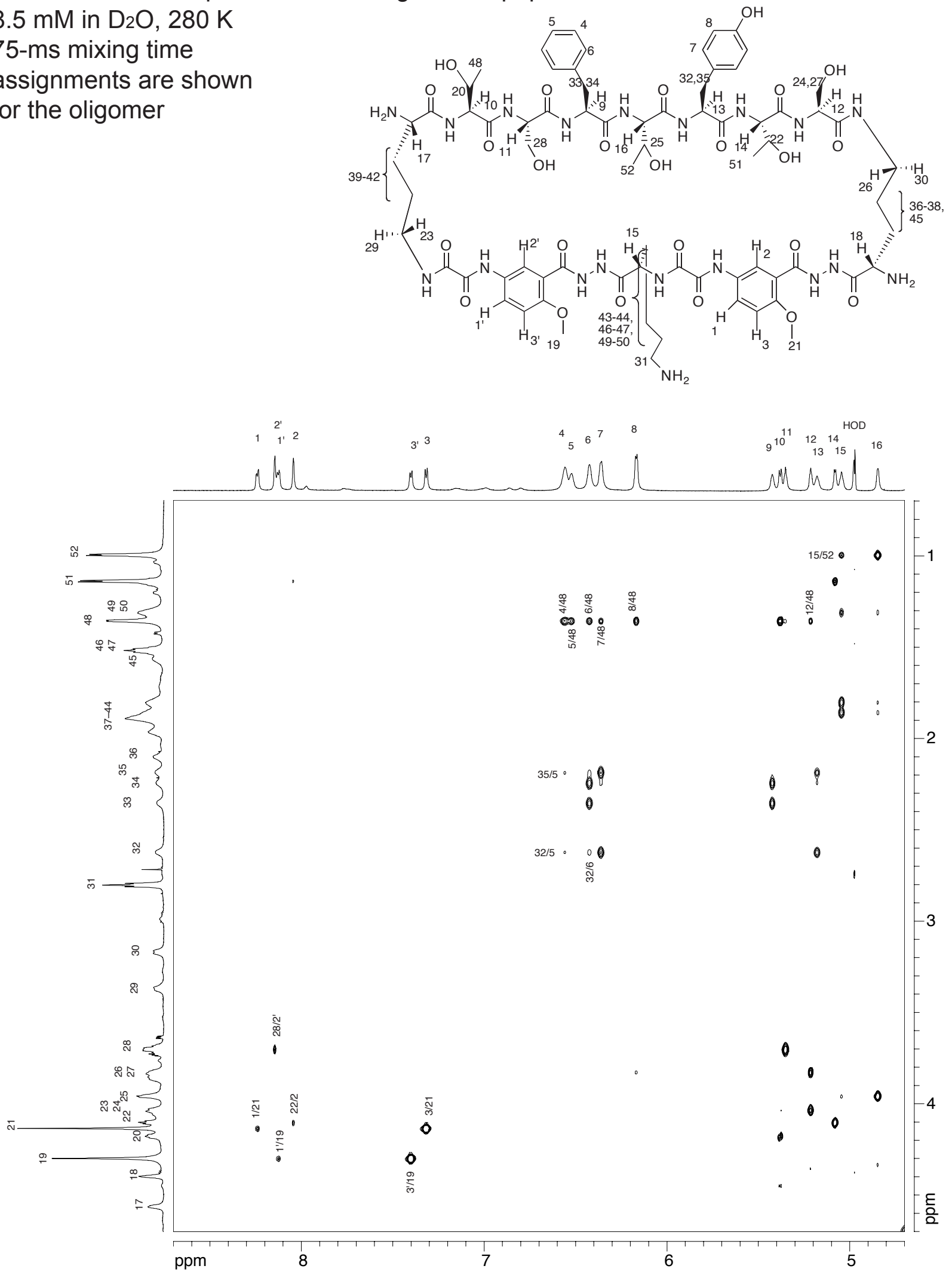
$800 \mathrm{MHz}$ NOESY spectrum of the oligomer of peptide $3 \mathrm{a}$

$8.5 \mathrm{mM}$ in $\mathrm{D}_{2} \mathrm{O}, 280 \mathrm{~K}$

75-ms mixing time

assignments are shown

for the oligomer
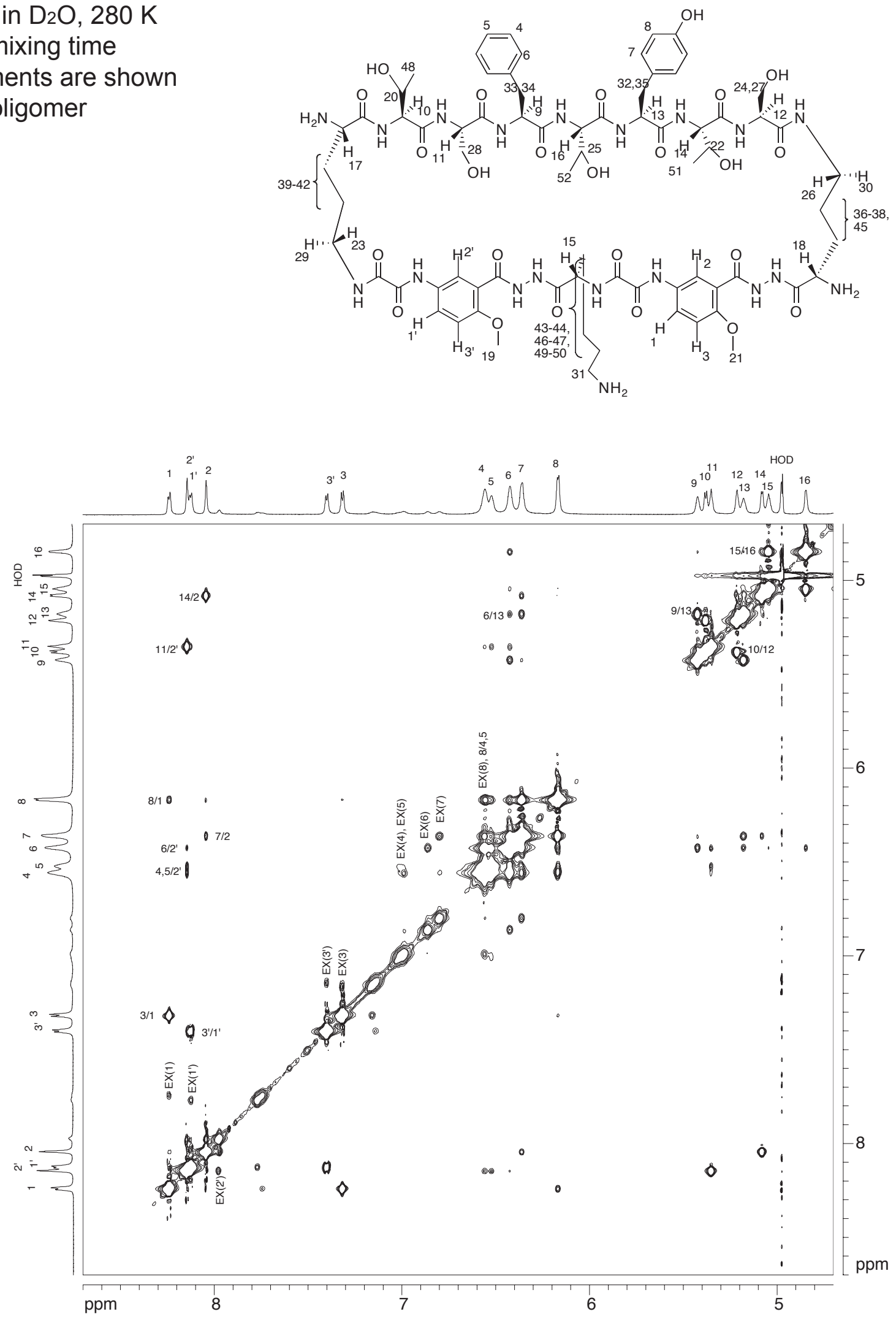
$800 \mathrm{MHz}$ NOESY spectrum of the oligomer of peptide $3 \mathbf{a}$

$8.5 \mathrm{mM}$ in $\mathrm{D}_{2} \mathrm{O}, 280 \mathrm{~K}$

75-ms mixing time

assignments are shown

for the oligomer

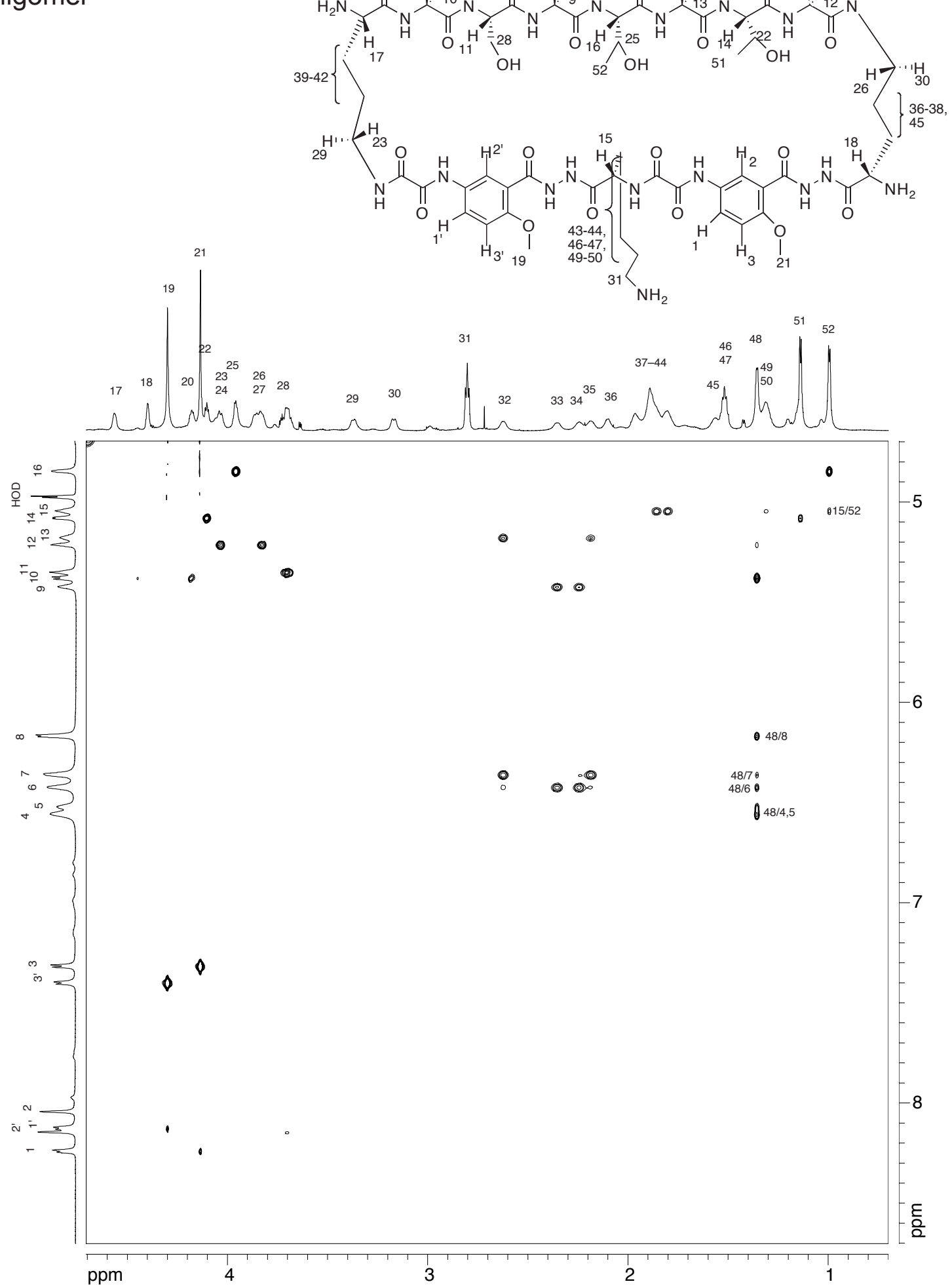


$500 \mathrm{MHz}$ ROESY spectrum of the oligomer of peptide 3a $8.5 \mathrm{mM}$ in $\mathrm{D}_{2} \mathrm{O}, 278 \mathrm{~K}$ 300-ms mixing time assignments are shown for the oligomer

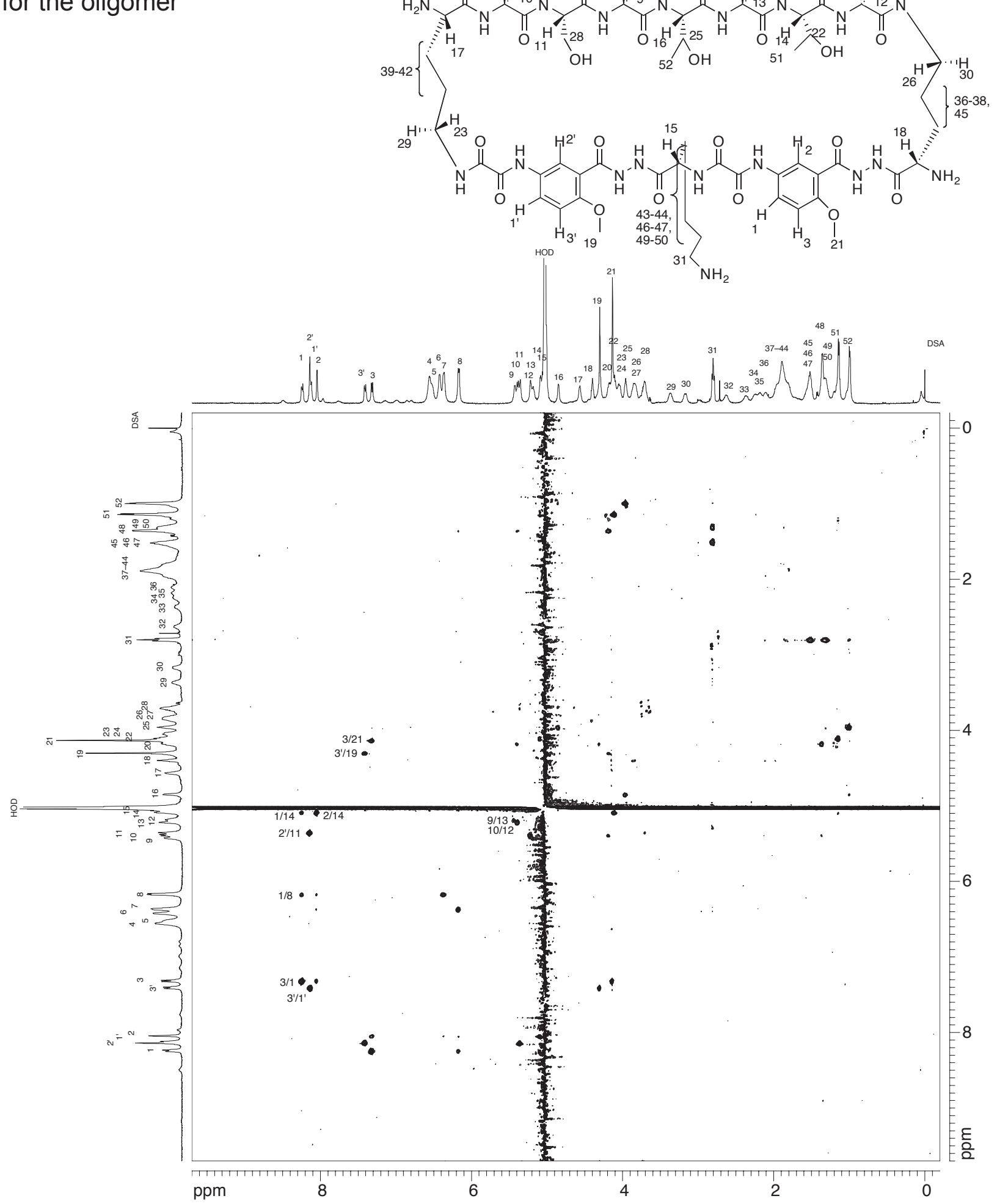



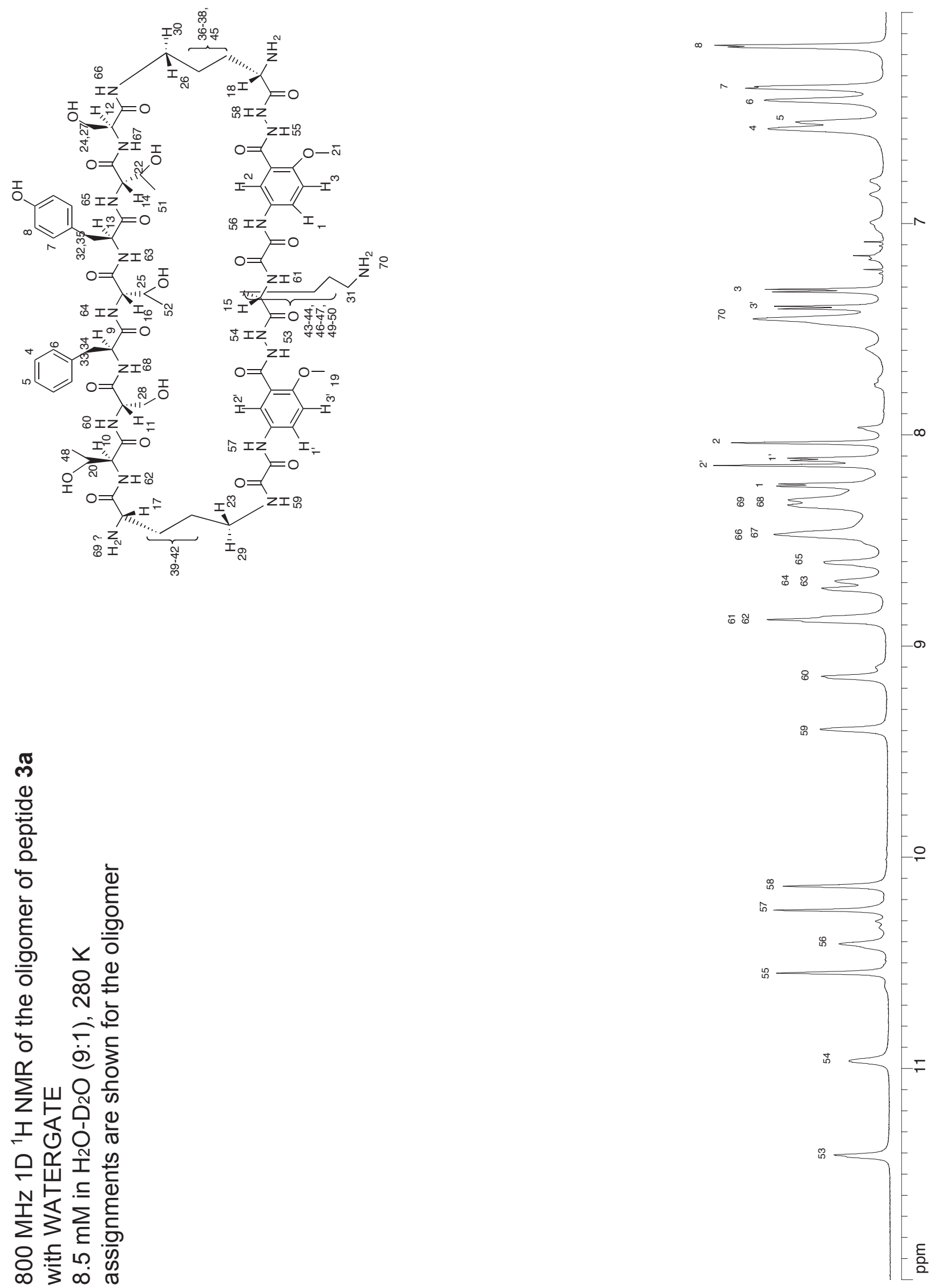
$800 \mathrm{MHz}$ TOCSY spectrum of the oligomer of peptide $3 \mathrm{a}$ with WATERGATE

$8.5 \mathrm{mM}$ in $\mathrm{H}_{2} \mathrm{O}-\mathrm{D}_{2} \mathrm{O}$ (9:1), $280 \mathrm{~K}$ 50 -ms spin-lock mixing time assignments are shown for the oligomer
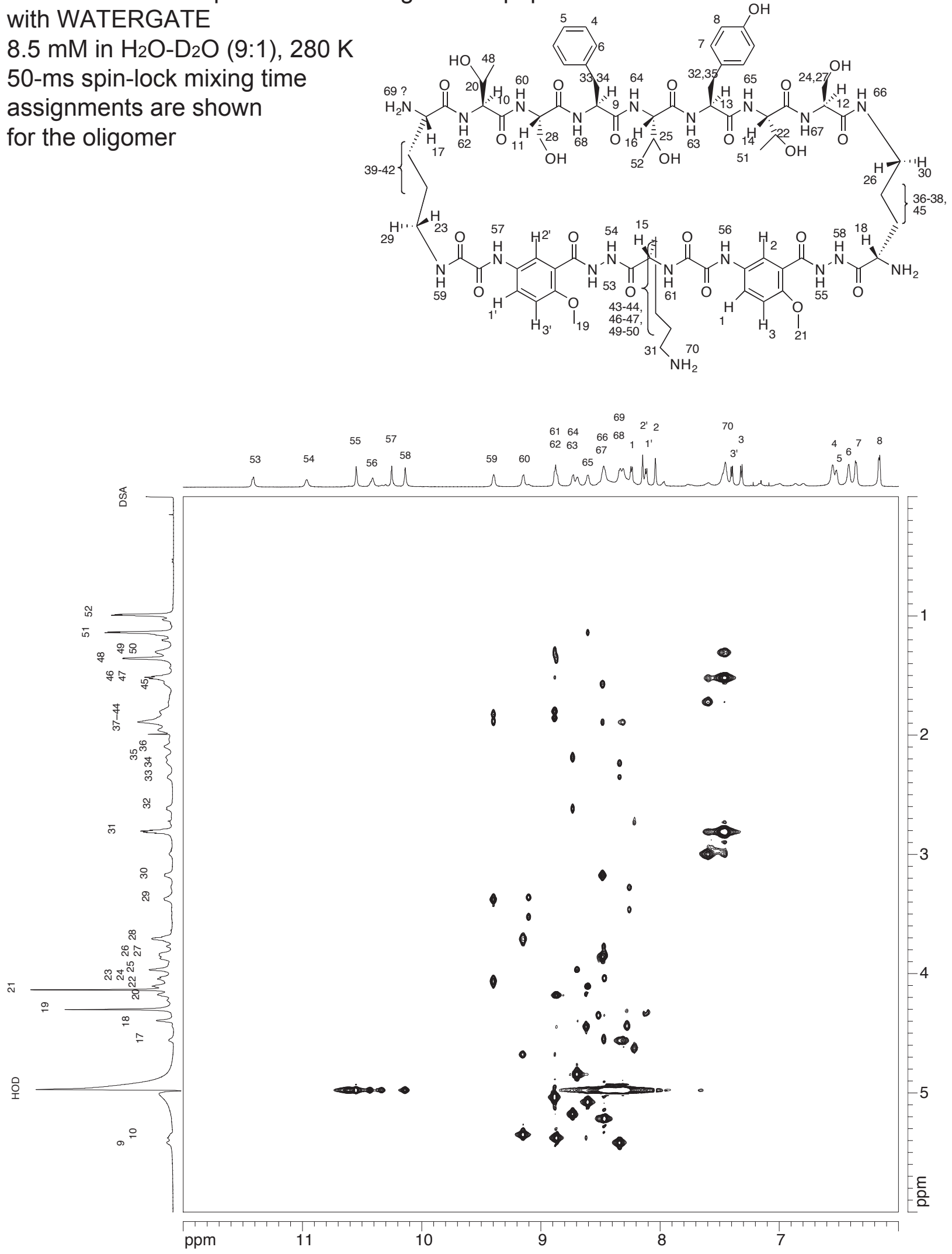
$800 \mathrm{MHz}$ TOCSY spectrum of the oligomer of peptide $3 \mathrm{a}$ with WATERGATE

$8.5 \mathrm{mM}$ in $\mathrm{H}_{2} \mathrm{O}-\mathrm{D}_{2} \mathrm{O}$ (9:1), $280 \mathrm{~K}$ 50-ms spin-lock mixing time assignments are shown for the oligomer
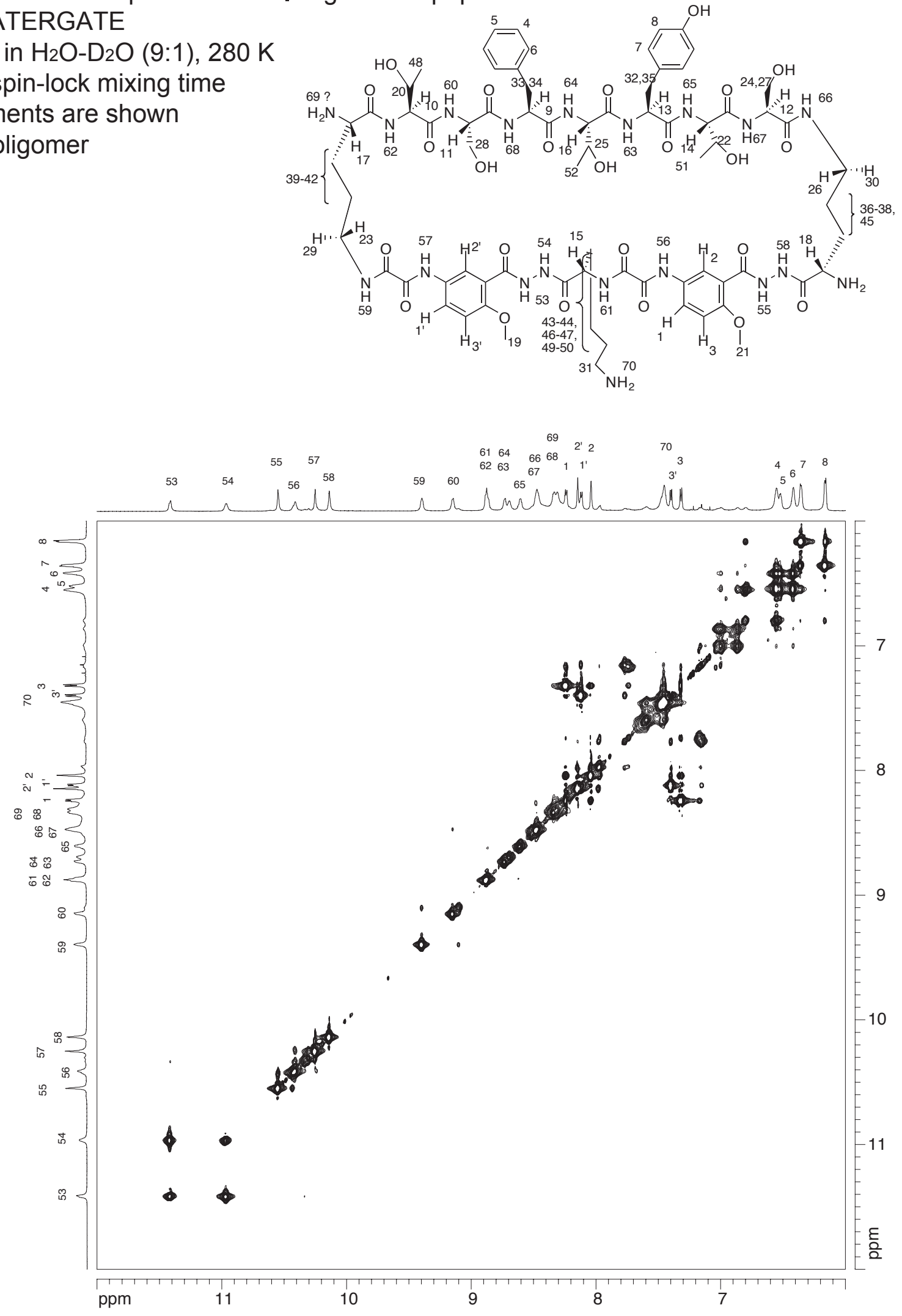
$800 \mathrm{MHz}$ NOESY spectrum of the oligomer of peptide $3 a$ with WATERGATE $8.5 \mathrm{mM}$ in $\mathrm{H}_{2} \mathrm{O}-\mathrm{D}_{2} \mathrm{O}(9: 1), 280 \mathrm{~K}$ 75-ms mixing time assignments are shown for the oligomer
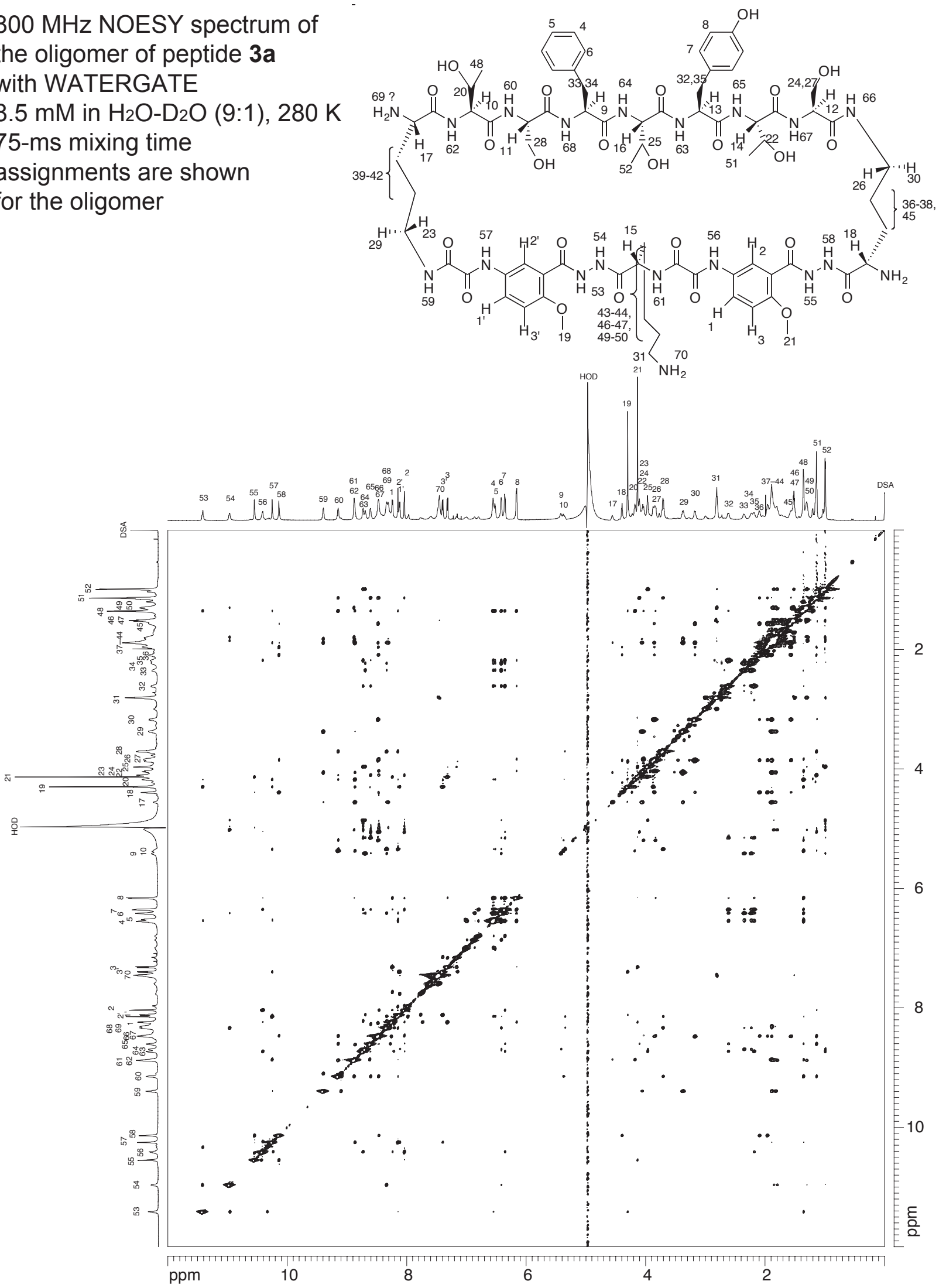
$800 \mathrm{MHz}$ NOESY spectrum of the oligomer of peptide $\mathbf{3 a}$ with WATERGATE $8.5 \mathrm{mM}$ in $\mathrm{H}_{2} \mathrm{O}-\mathrm{D}_{2} \mathrm{O}(9: 1), 280 \mathrm{~K}$ 75-ms mixing time assignments are shown for the oligomer
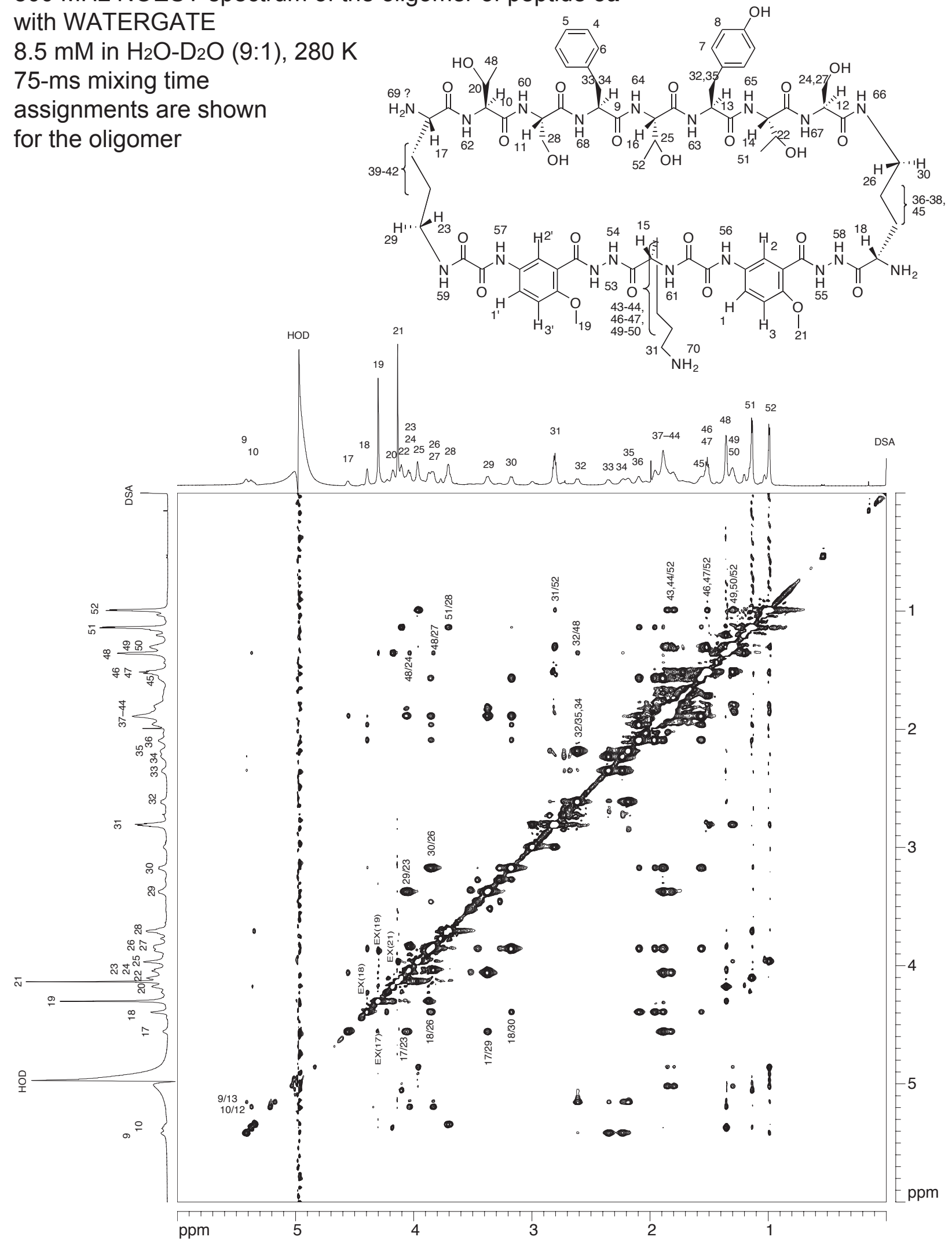
$800 \mathrm{MHz}$ NOESY spectrum of the oligomer of peptide $3 \mathrm{a}$ with WATERGATE

$8.5 \mathrm{mM}$ in $\mathrm{H}_{2} \mathrm{O}-\mathrm{D}_{2} \mathrm{O}(9: 1), 280 \mathrm{~K}$ 75-ms mixing time assignments are shown for the oligomer
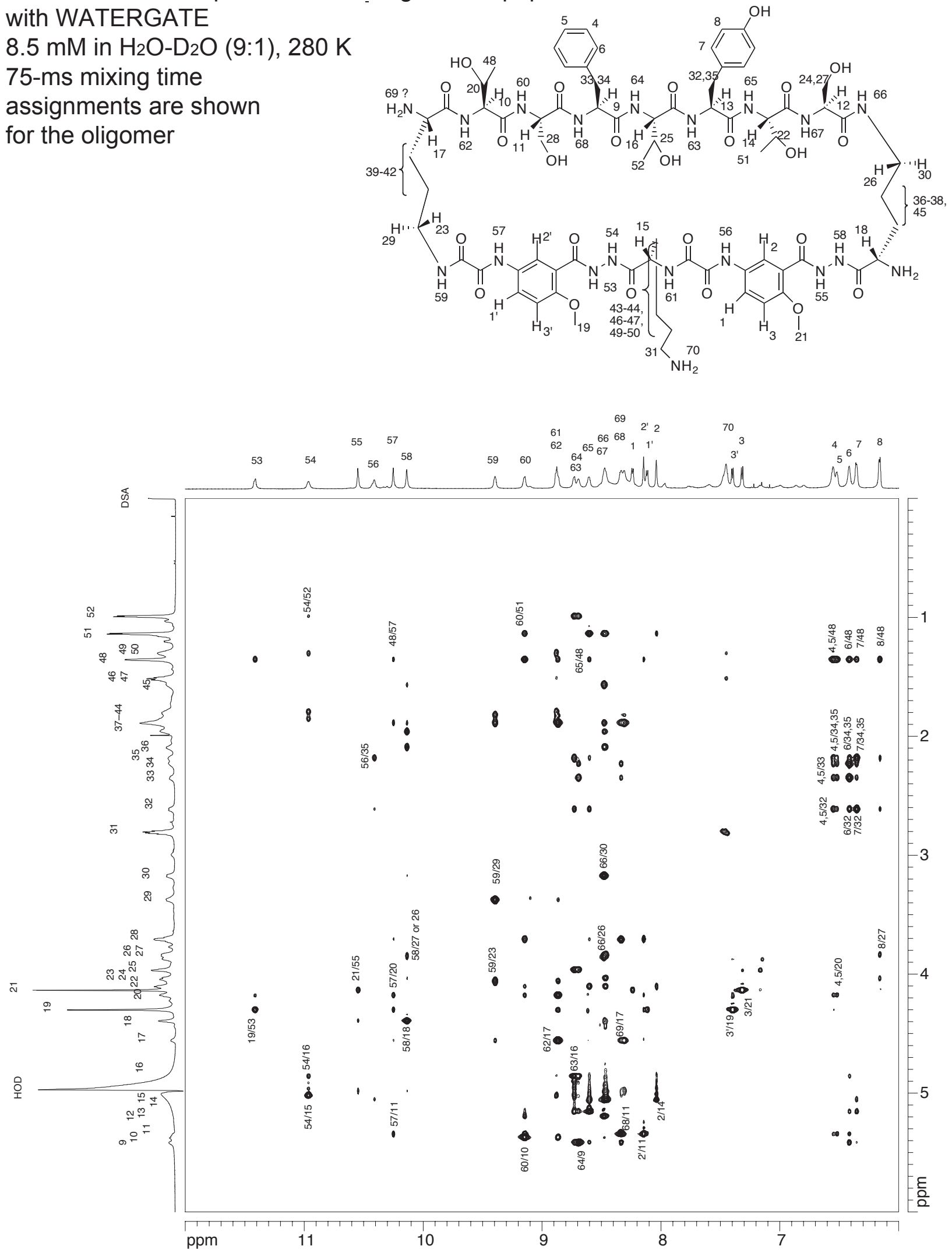
$800 \mathrm{MHz}$ NOESY spectrum of the oligomer of peptide $\mathbf{3 a}$ with WATERGATE $8.5 \mathrm{mM}$ in $\mathrm{H}_{2} \mathrm{O}-\mathrm{D}_{2} \mathrm{O}(9: 1), 280 \mathrm{~K}$ 75-ms mixing time $\mathrm{NH}-\mathrm{C}$ protons region of a higher contour level, emphasizing intraresidue and $(\mathrm{i}, \mathrm{i}+1)$ interresidue $\mathrm{NH}-\mathrm{C}$ proton NOEs
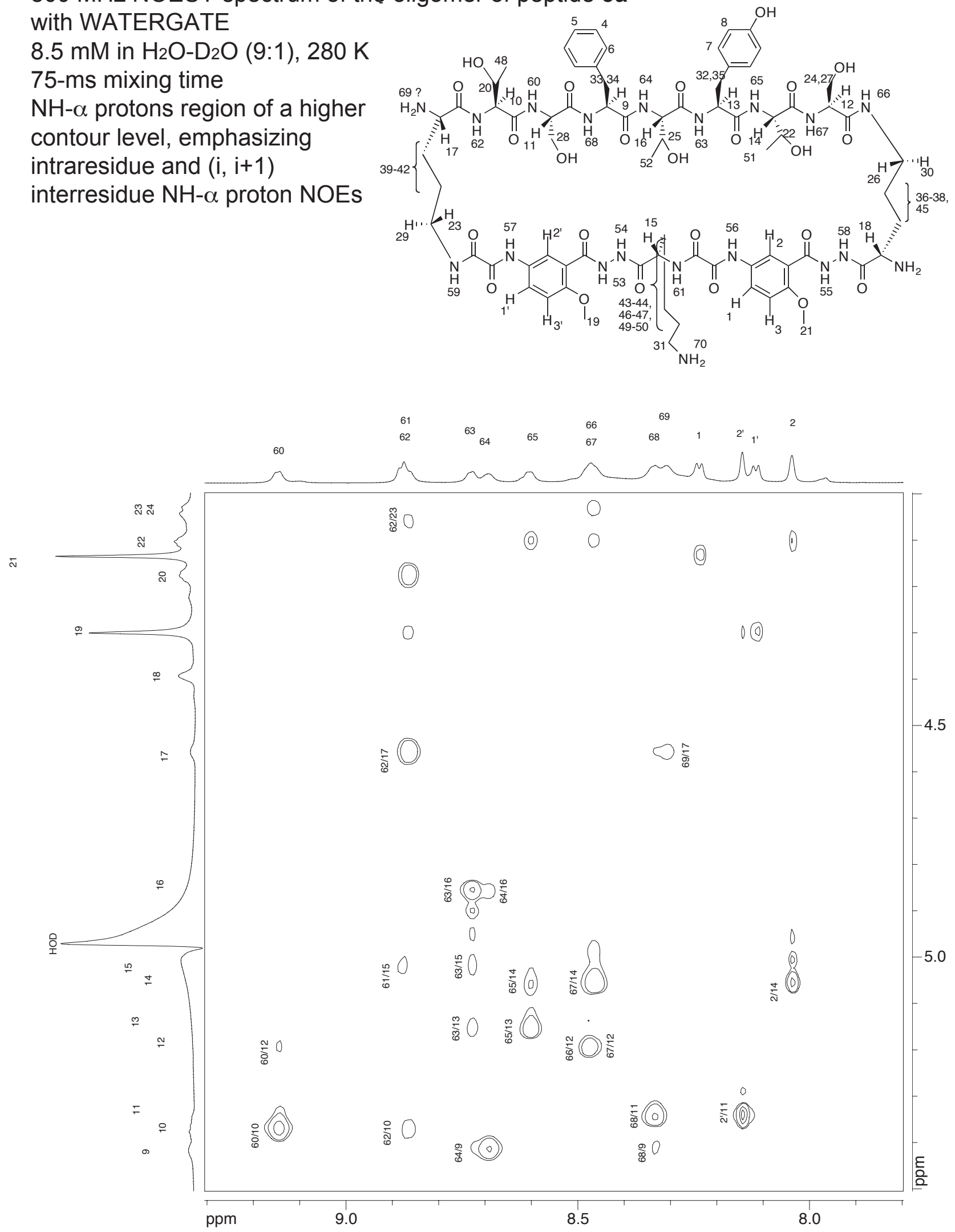
$800 \mathrm{MHz}$ NOESY spectrum of the oligomer of peptide $\mathbf{3 a}$ with WATERGATE

$8.5 \mathrm{mM}$ in $\mathrm{H}_{2} \mathrm{O}-\mathrm{D}_{2} \mathrm{O}(9: 1), 280 \mathrm{~K}$ 75-ms mixing time assignments are shown for the oligomer

EXSY cross peaks from the chemical exchange between the monomer and the oligomer are also observed here.
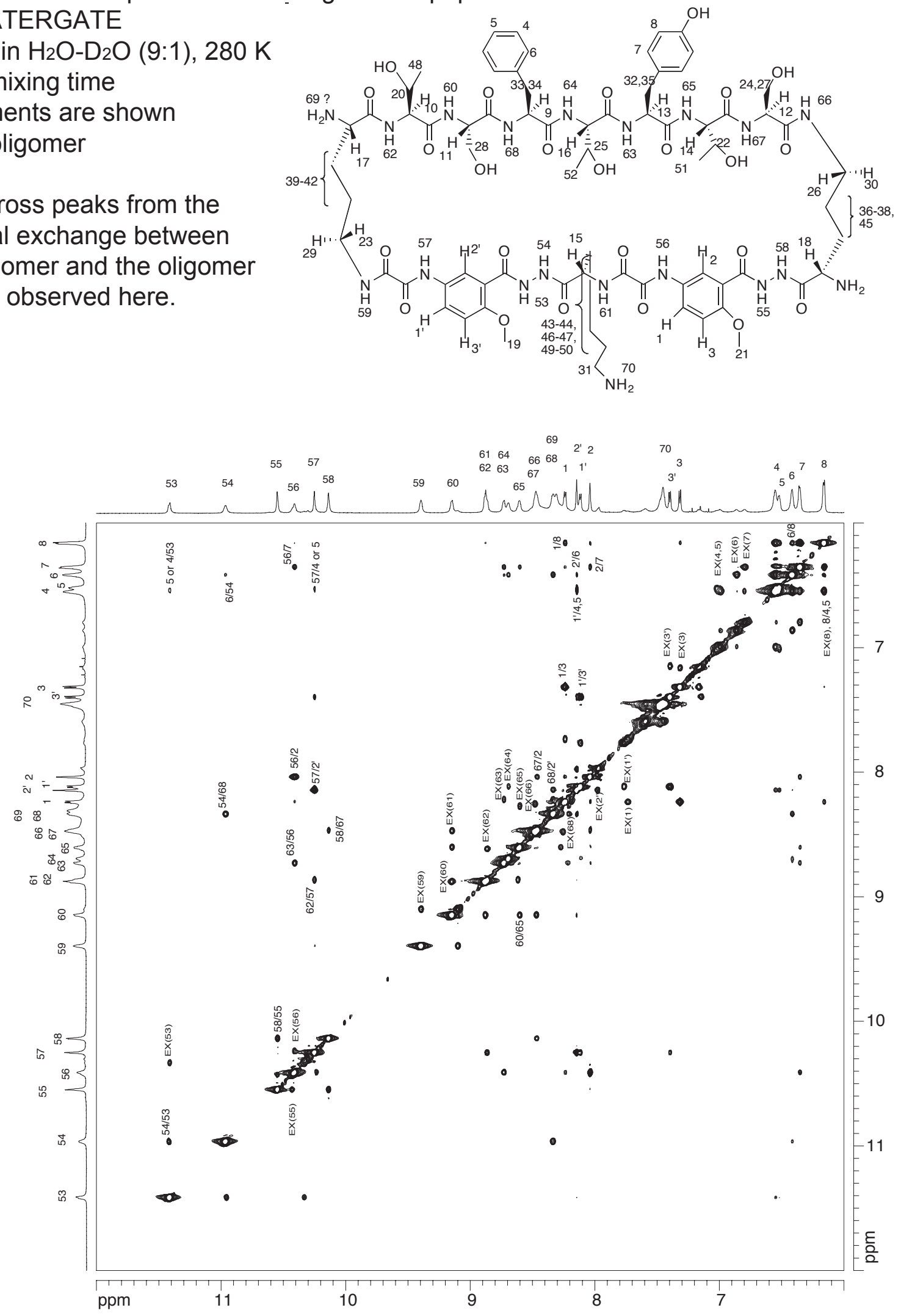
$800 \mathrm{MHz}$ NOESY spectrum of the oligomer of peptide $\mathbf{3 a}$ with WATERGATE $8.5 \mathrm{mM}$ in $\mathrm{H}_{2} \mathrm{O}-\mathrm{D}_{2} \mathrm{O}(9: 1), 280 \mathrm{~K}$ 75-ms mixing time assignments are shown for the oligomer

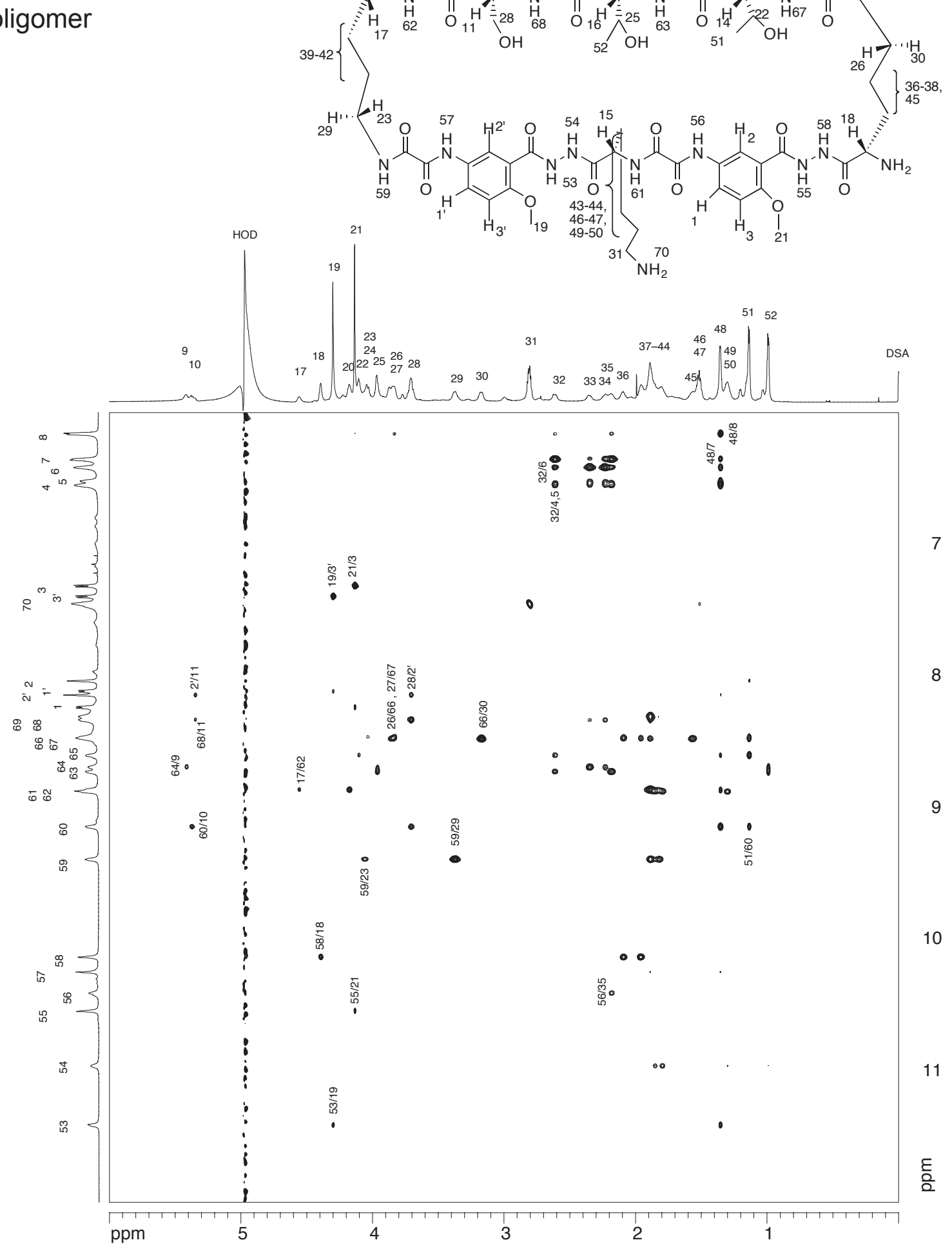


$800 \mathrm{MHz}$ EXSY spectrum of the oligomer of peptide $\mathbf{3 a}$ with WATERGATE $8.5 \mathrm{mM}$ in $\mathrm{H}_{2} \mathrm{O}-\mathrm{D}_{2} \mathrm{O}(9: 1), 280 \mathrm{~K}$ 50 -ms mixing time assignments are shown for the oligomer

EXSY cross-peaks are originating from the chemical exchange between the monomer and the oligomer
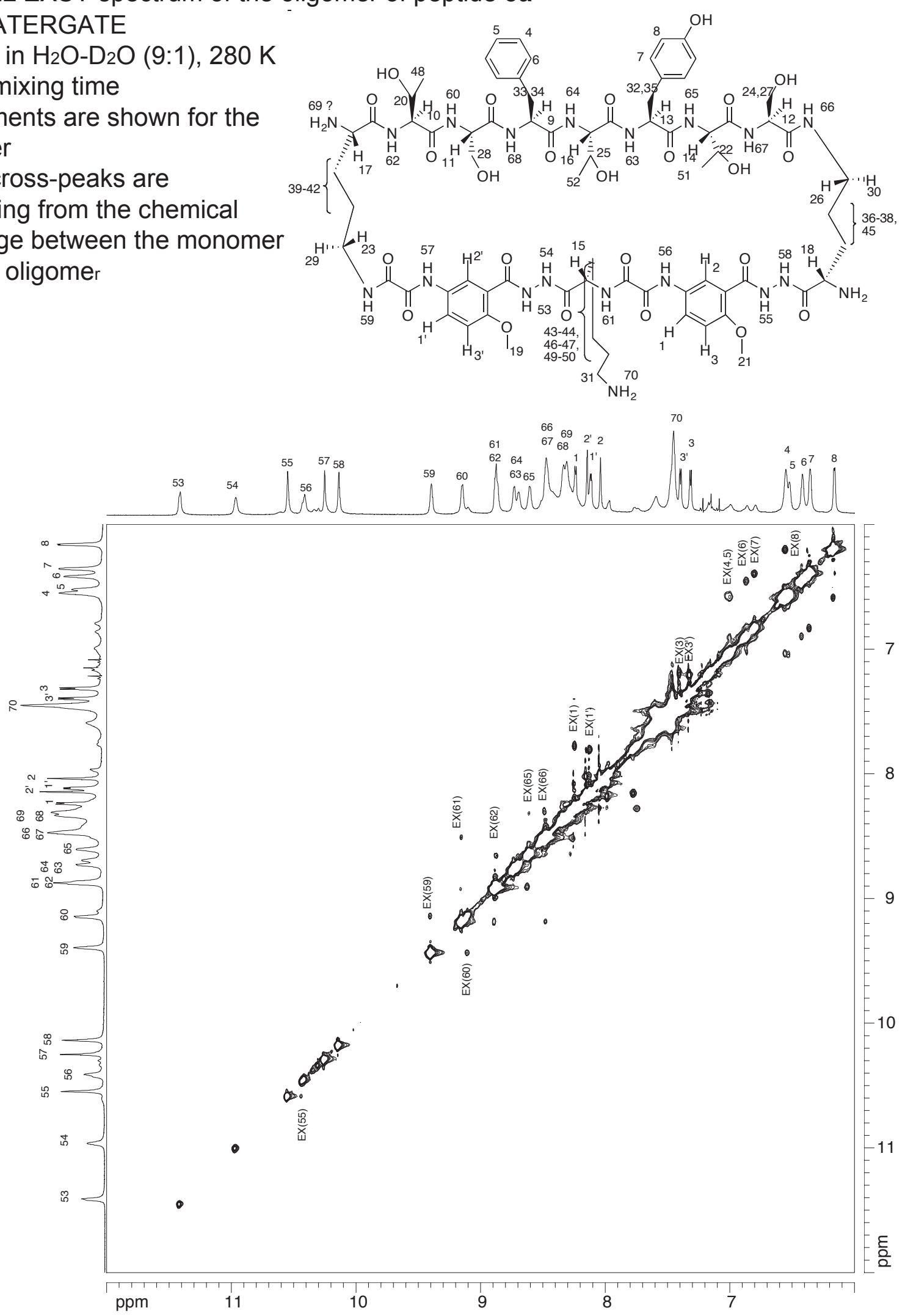
Key NOEs in the oligomer of peptide $\mathbf{3 a}$

a. Interstrand main chain-main chain NOEs

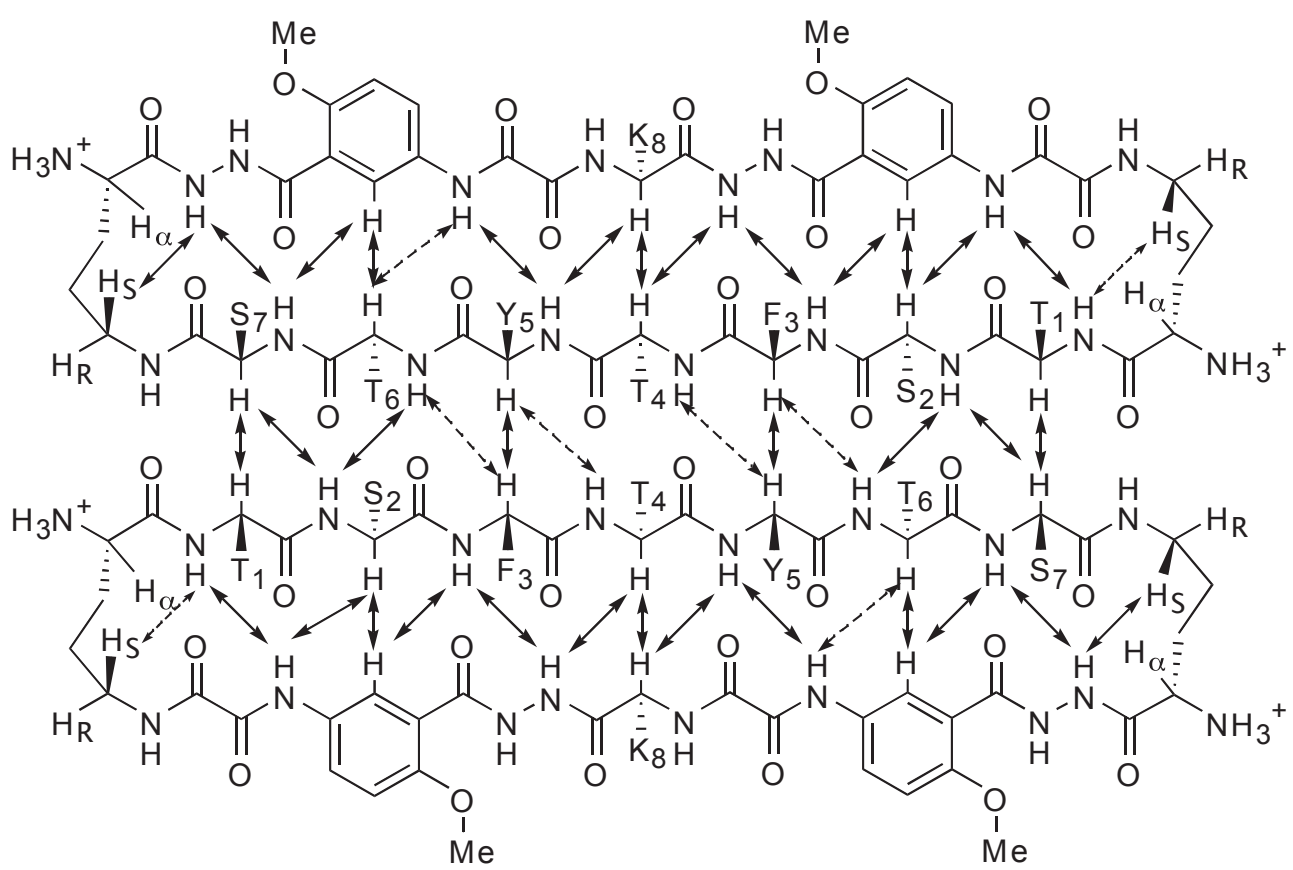

b. side chain-side chain NOEs

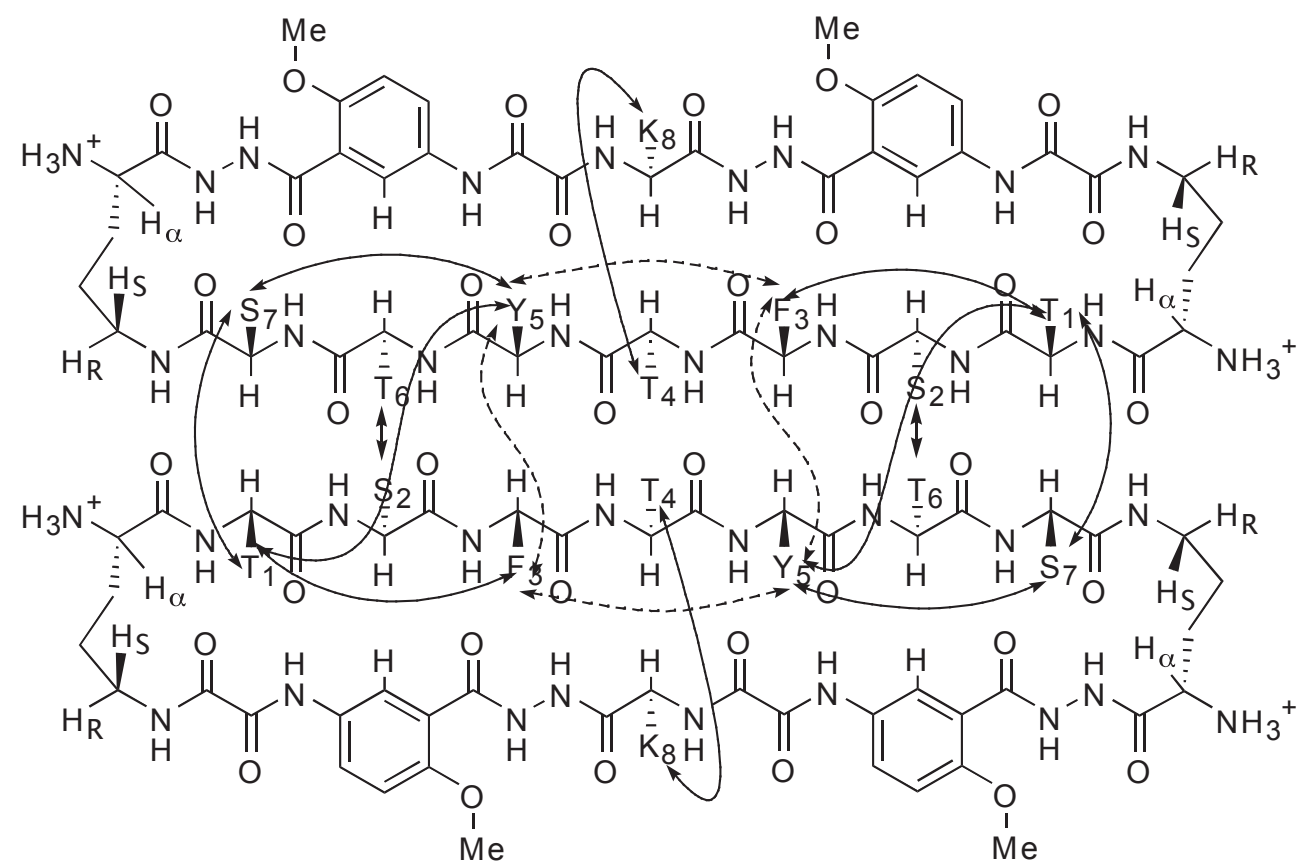

dashed arrows represent the weak or ambiguous NOEs 
Key NOEs in the oligomer of peptide $\mathbf{3 a}$

c. Interstrand side chain-main chain NOEs

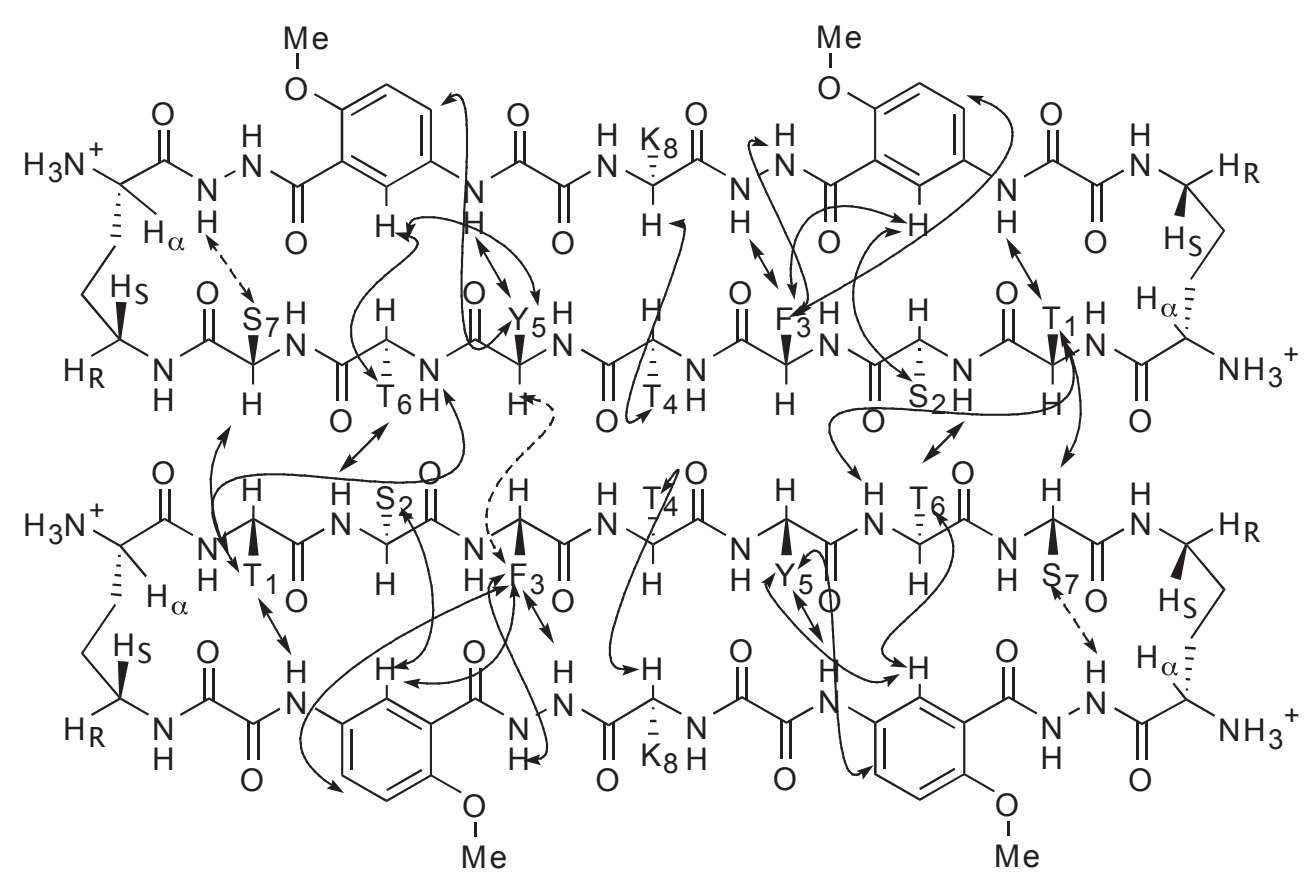

dashed arrows represent the weak or ambiguous NOEs

d. Intrastrand and turn NOEs

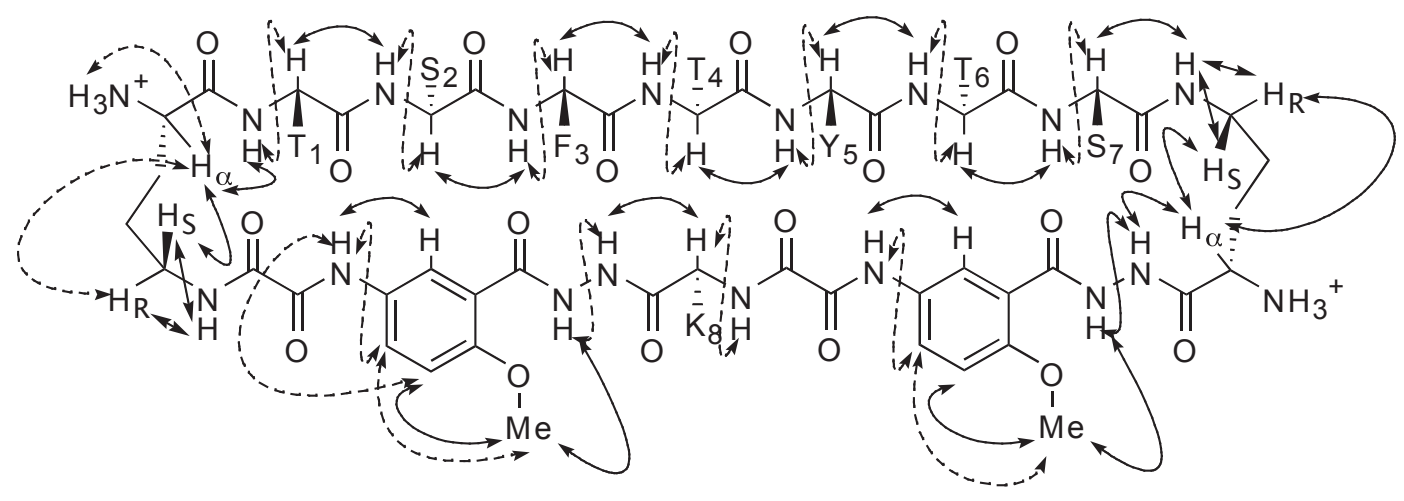

dashed arrows represent the weaker NOEs 

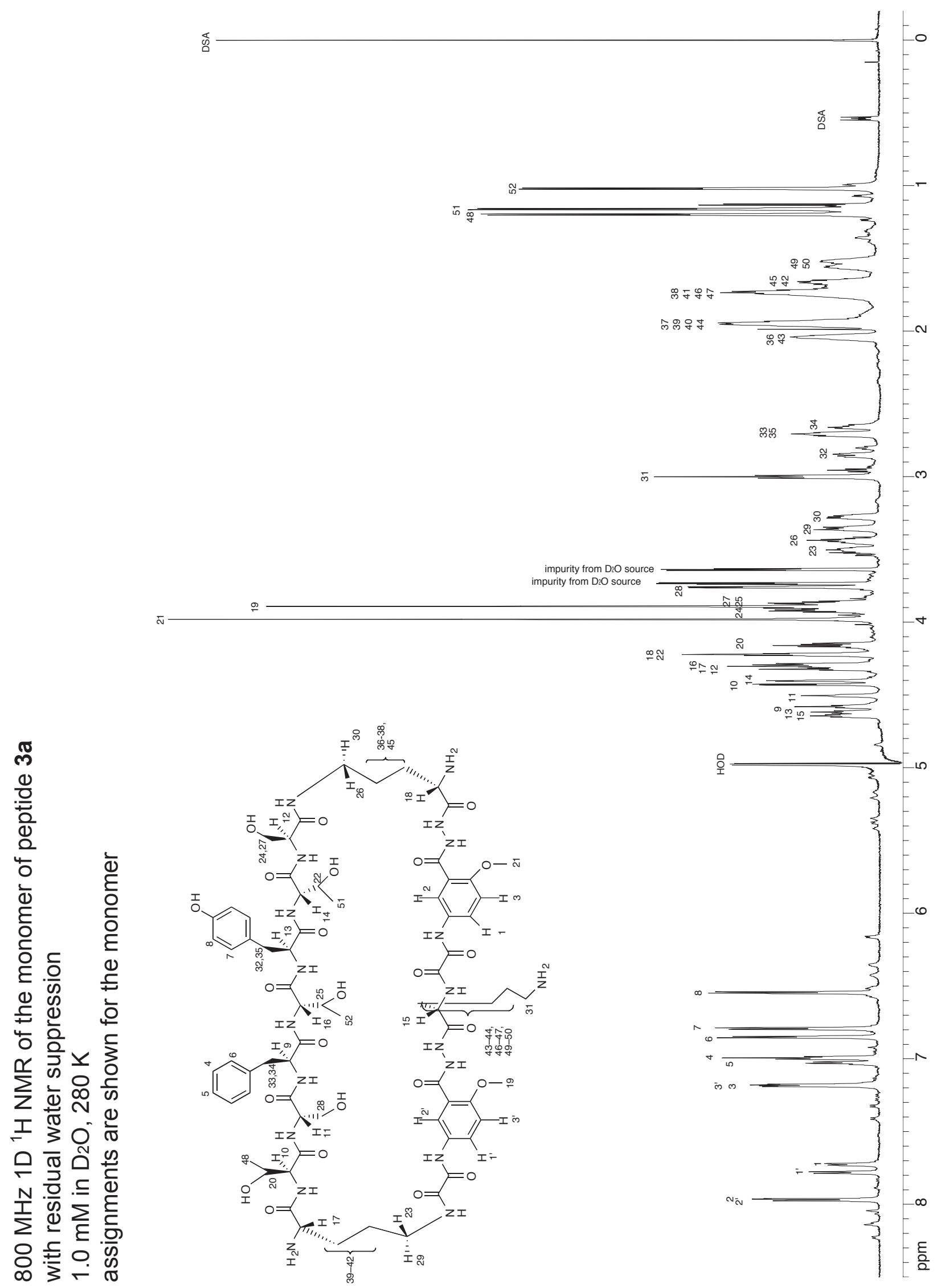
$800 \mathrm{MHz} 1 \mathrm{D}{ }^{1} \mathrm{H}$ NMR of the monomer of peptide $\mathbf{3 a}$ with residual water suppression

$1.0 \mathrm{mM}$ in $\mathrm{D}_{2} \mathrm{O}, 280 \mathrm{~K}$ assignments are shown for the monomer
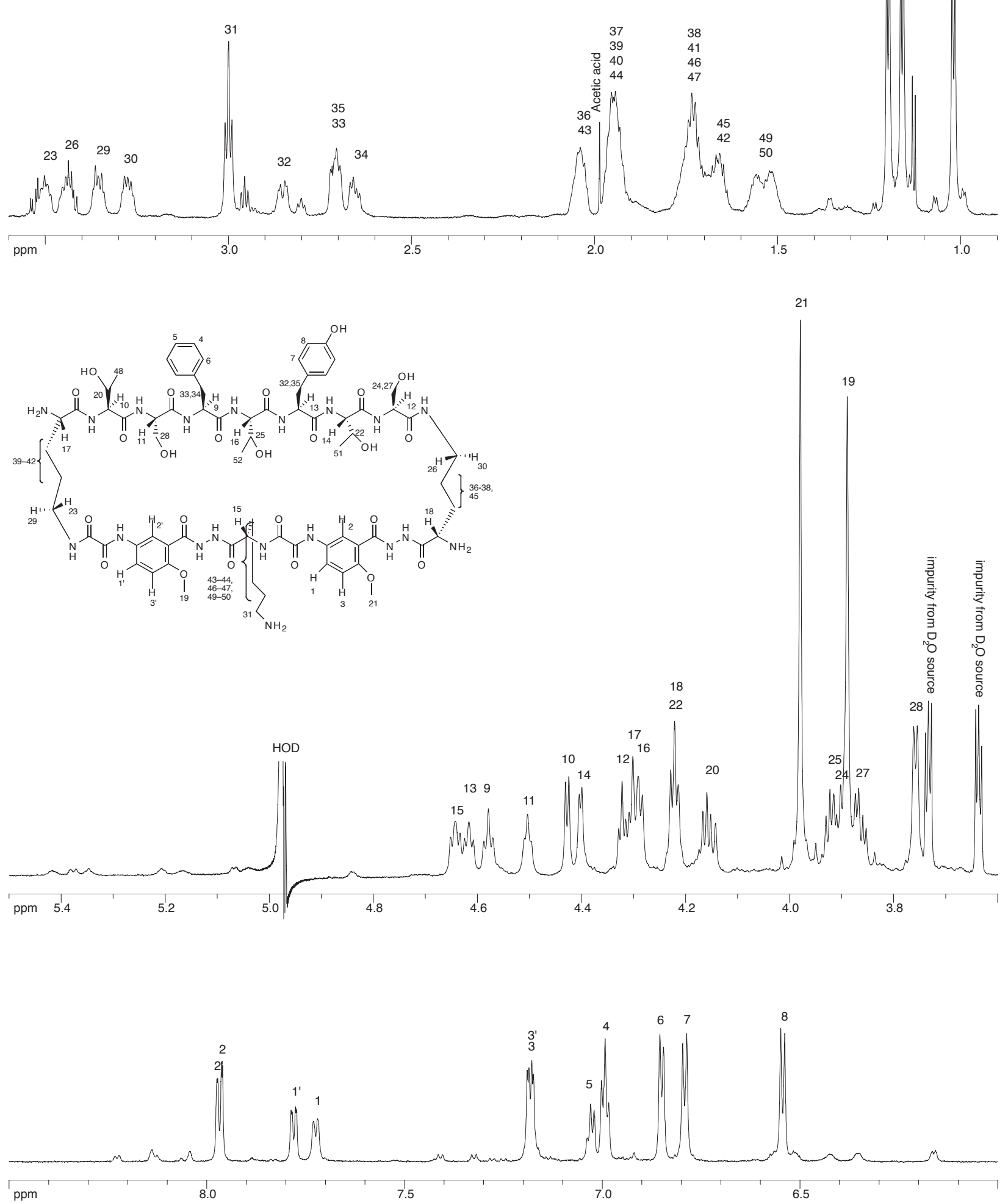
$800 \mathrm{MHz}$ TOCSY spectrum of the monomer of peptide $\mathbf{3 a}$ (50-ms mixing time) $1.0 \mathrm{mM}$ in $\mathrm{D}_{2} \mathrm{O}, 280 \mathrm{~K}$ with residual water suppression assignments are shown for the monomer
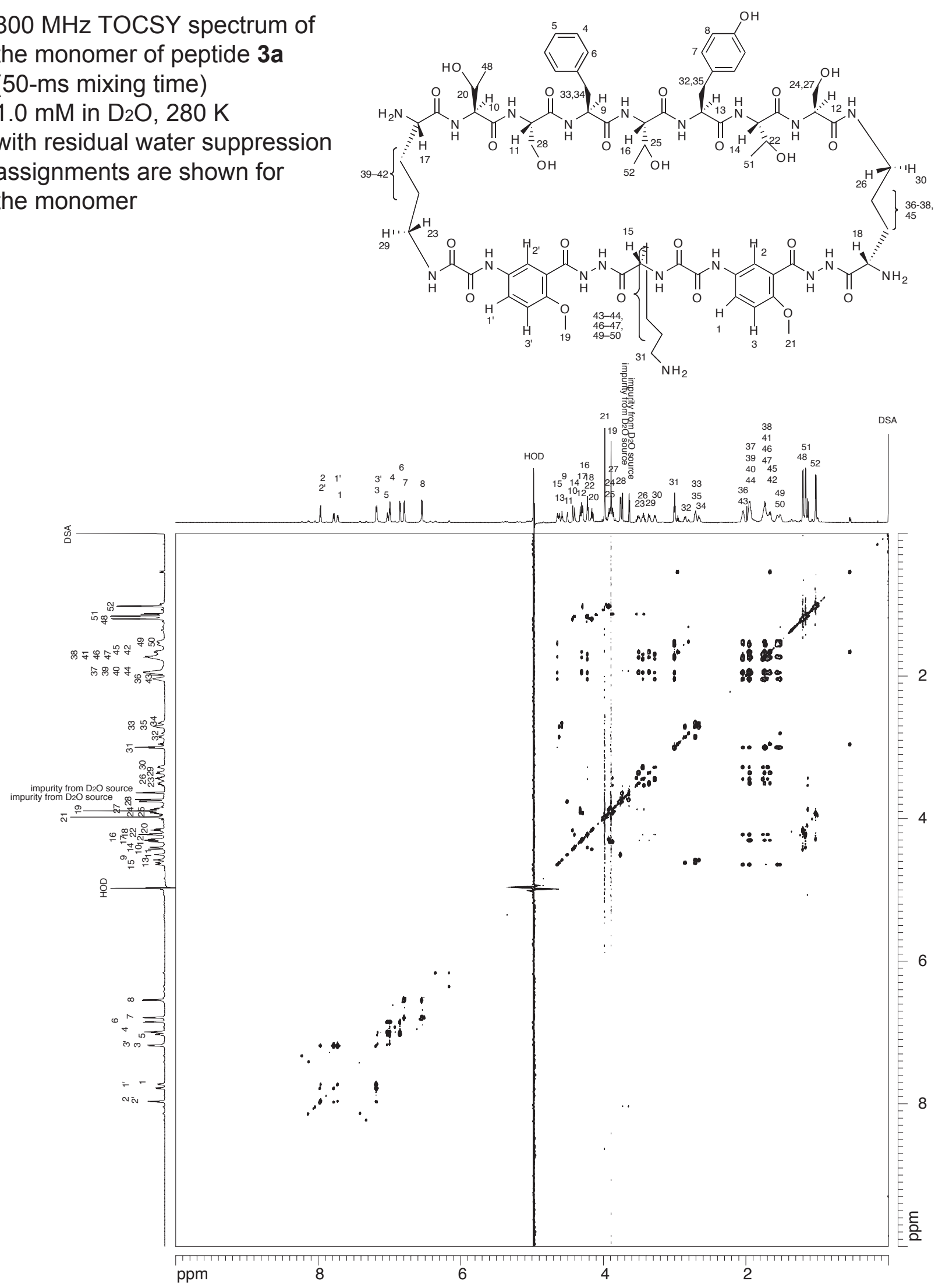

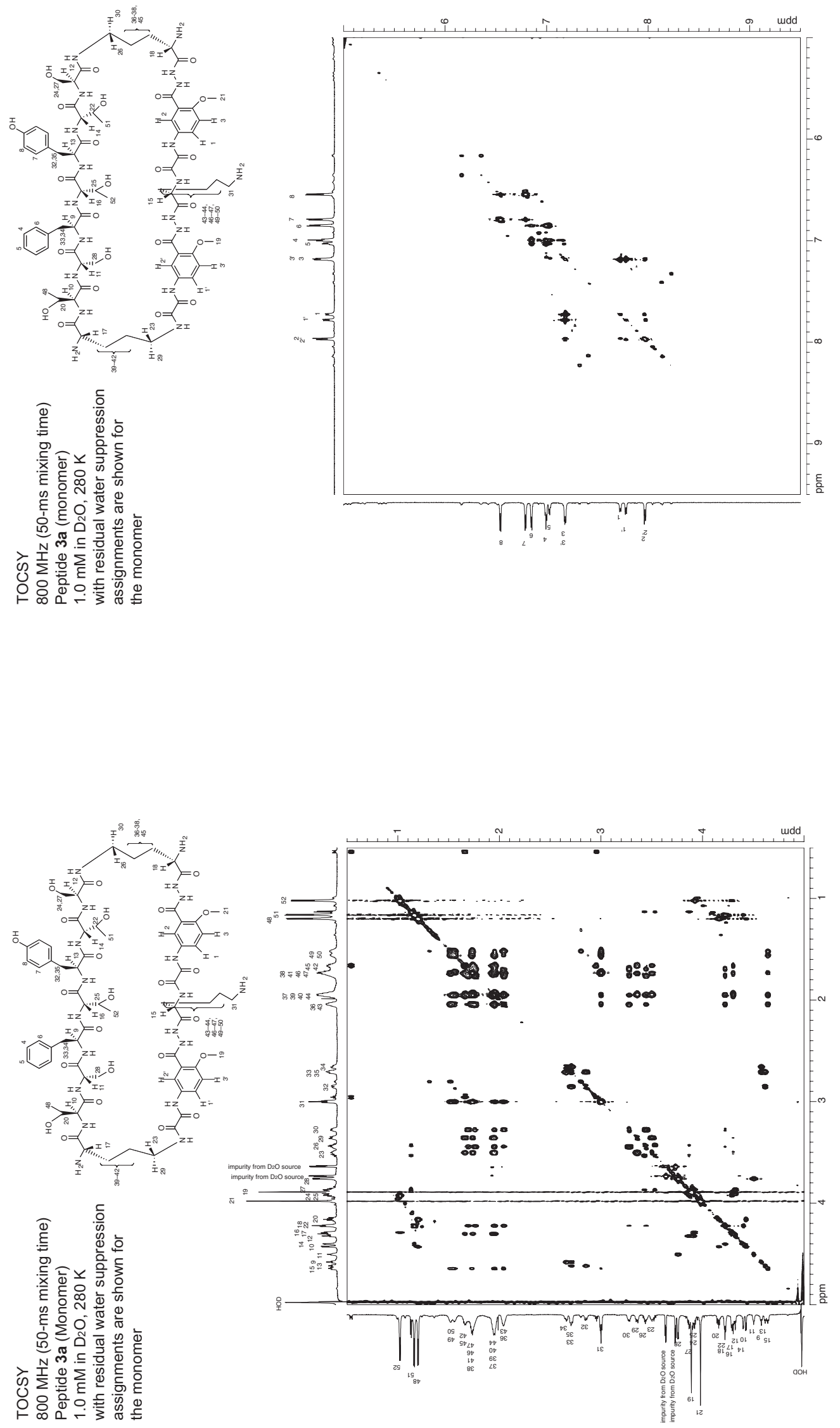
$800 \mathrm{MHz}$ NOESY spectrum of the monomer of peptide $\mathbf{3 a}$ (400-ms mixing time) $1.0 \mathrm{mM}$ in $\mathrm{D}_{2} \mathrm{O}, 280 \mathrm{~K}$ with residual water suppression assignments are shown for the monomer
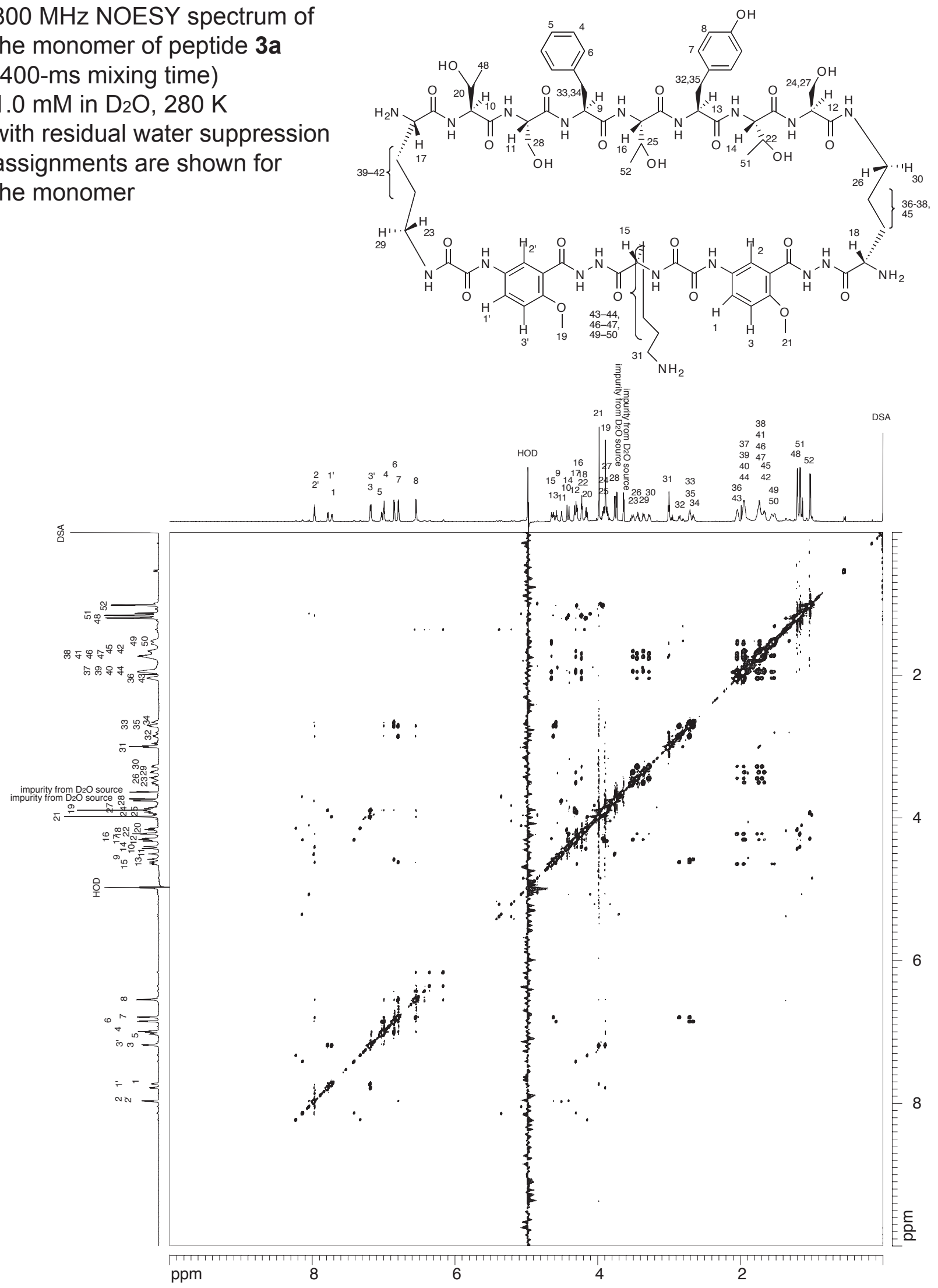
$800 \mathrm{MHz}$ NOESY spectrum of the monomer of peptide $3 \mathbf{a}$ (400-ms mixing time) $1.0 \mathrm{mM}$ in $\mathrm{D}_{2} \mathrm{O}, 280 \mathrm{~K}$ with residual water suppression assignments are shown for the monomer
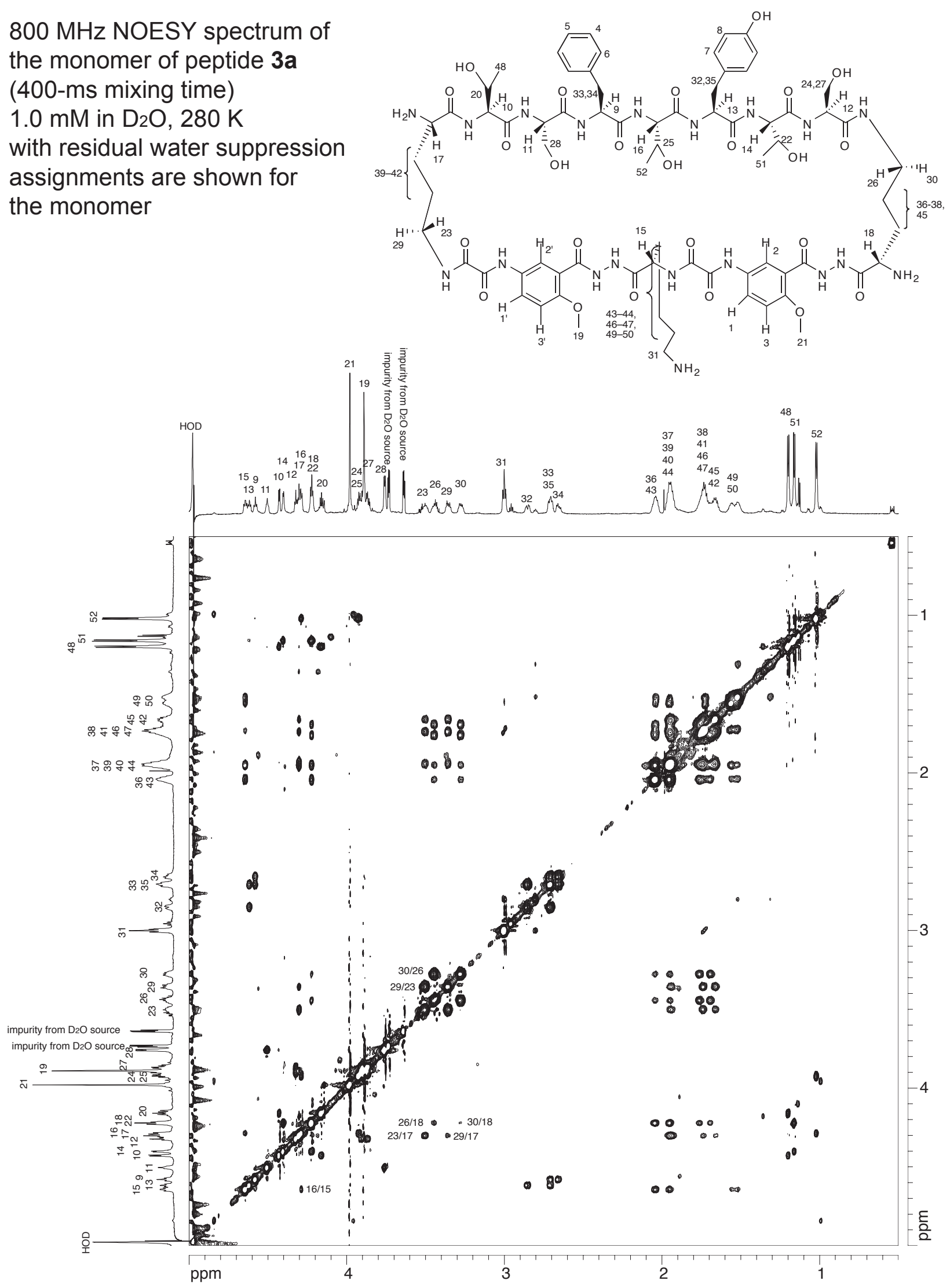
$800 \mathrm{MHz}$ NOESY spectrum of the monomer of peptide $\mathbf{3 a}$ (400-ms mixing time) $1.0 \mathrm{mM}$ in $\mathrm{D}_{2} \mathrm{O}, 280 \mathrm{~K}$ with residual water suppression assignments are shown for the monomer
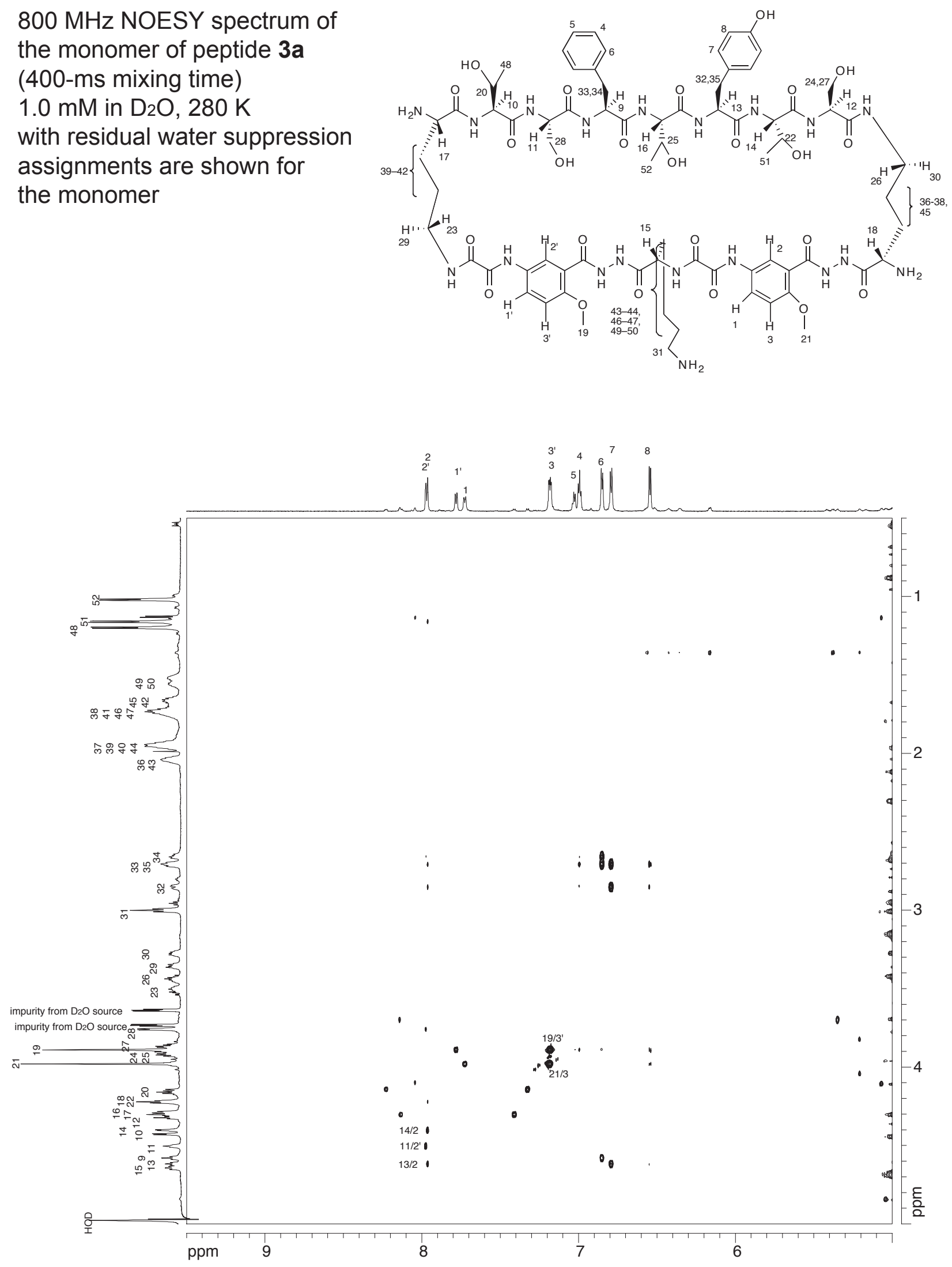
$800 \mathrm{MHz}$ NOESY spectrum of the monomer of peptide $\mathbf{3 a}$ (400-ms mixing time) $1.0 \mathrm{mM}$ in $\mathrm{D}_{2} \mathrm{O}, 280 \mathrm{~K}$ with residual water suppression assignments are shown for the monomer
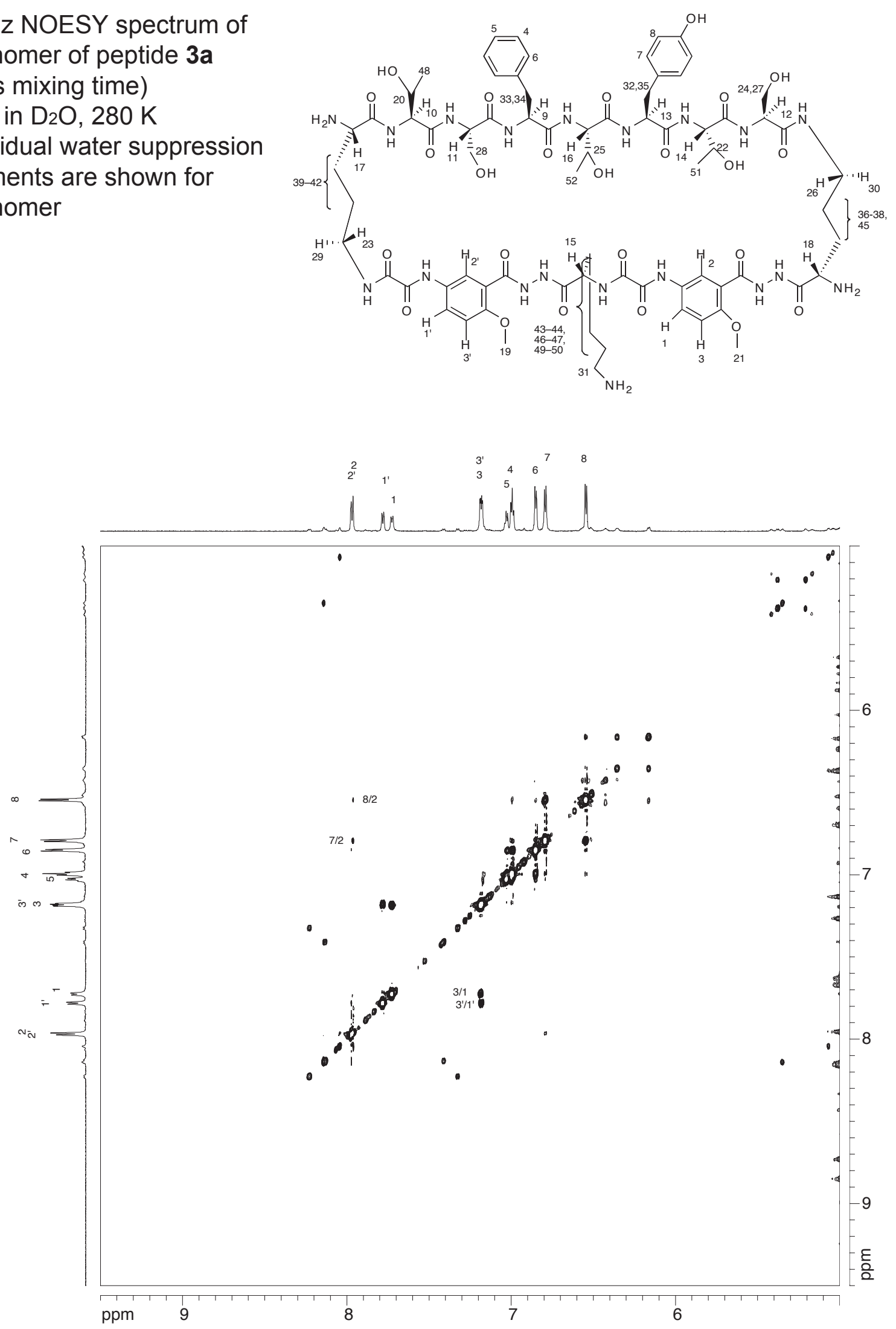
$800 \mathrm{MHz}$ NOESY spectrum of the monomer of peptide $\mathbf{3 a}$ (400-ms mixing time) $1.0 \mathrm{mM}$ in $\mathrm{D}_{2} \mathrm{O}, 280 \mathrm{~K}$ with residual water suppression assignments are shown for the monomer
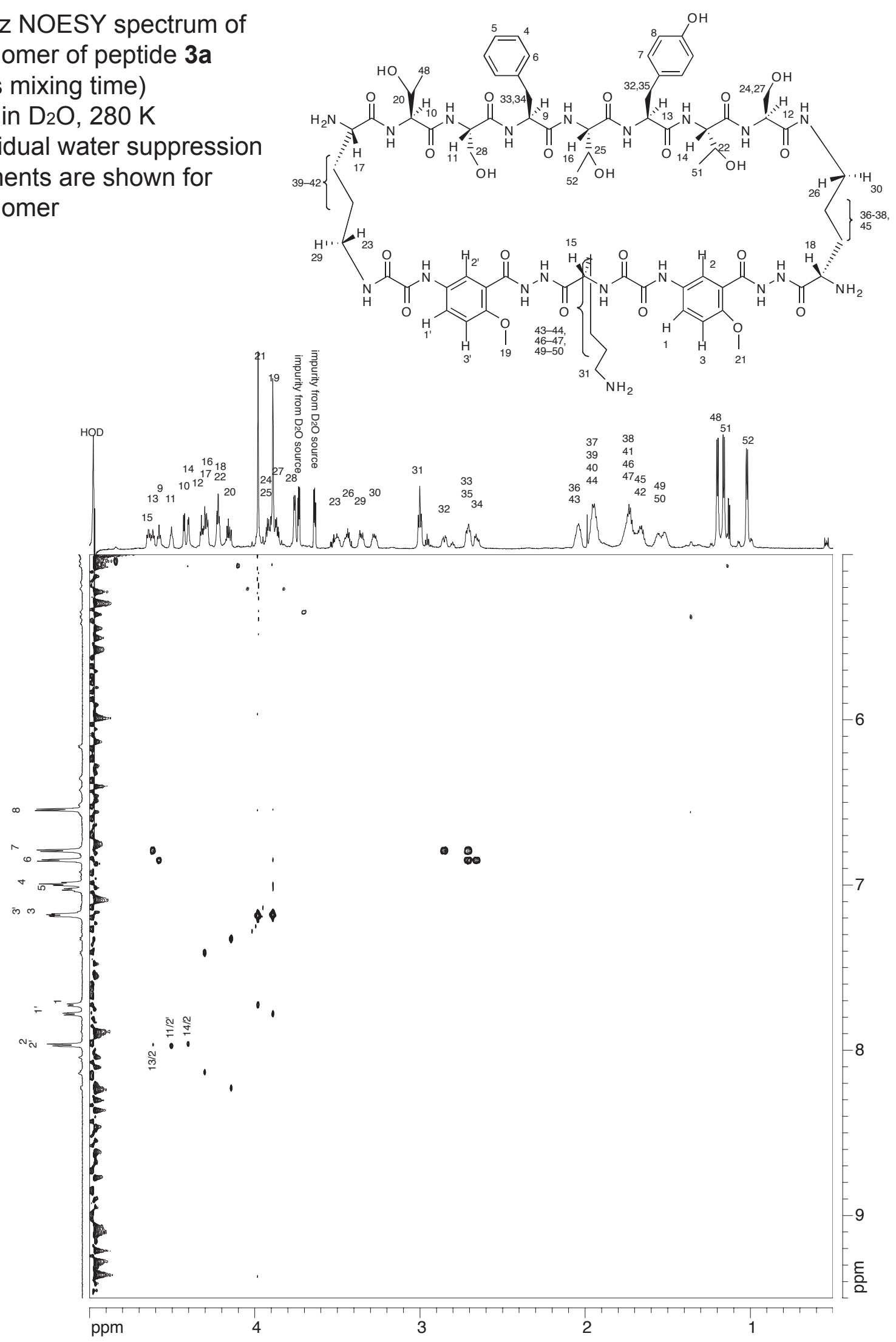

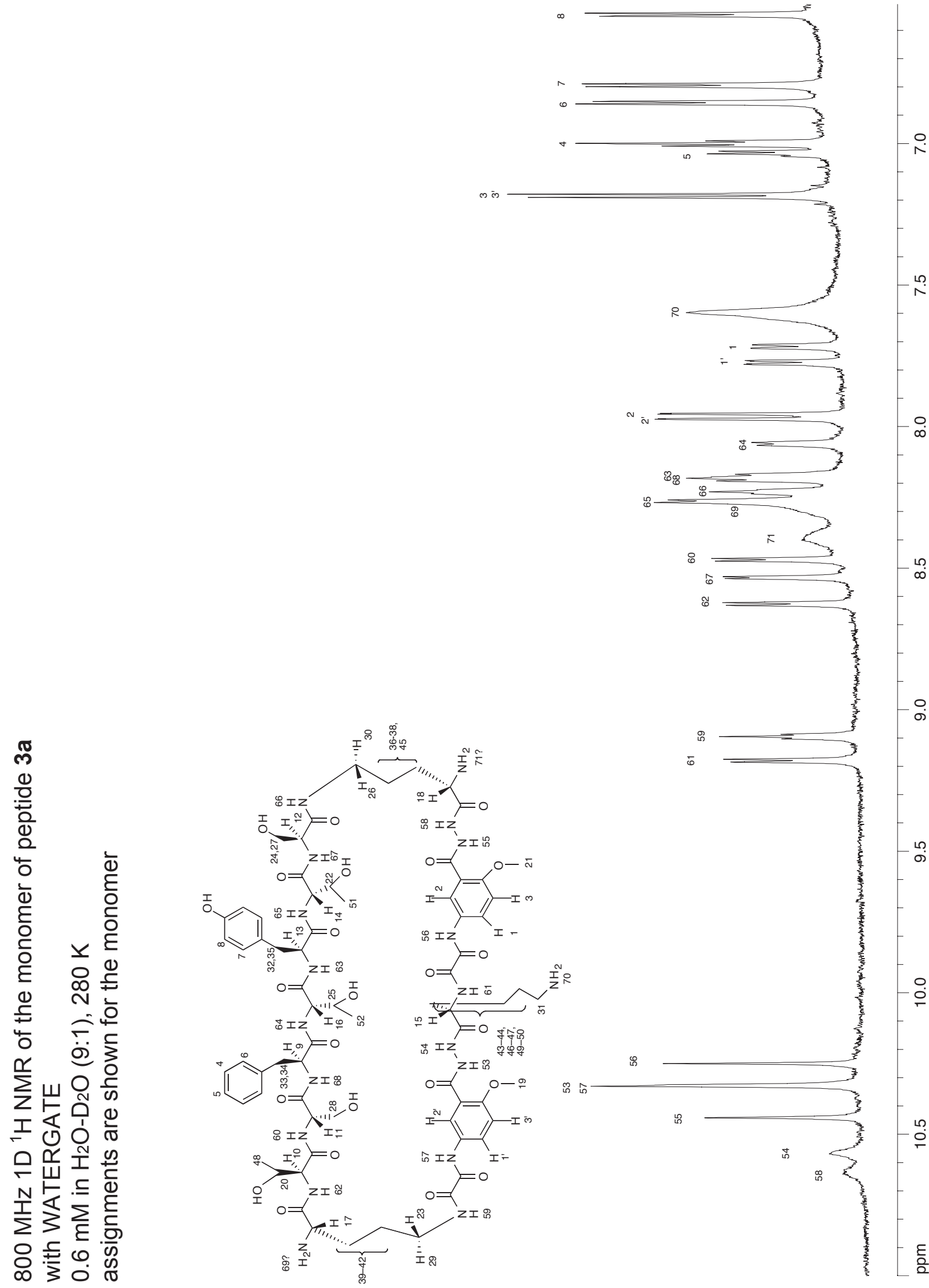
$800 \mathrm{MHz}$ TOCSY spectrum of the monomer of peptide 3a with WATERGATE $0.6 \mathrm{mM}$ in $\mathrm{H}_{2} \mathrm{O}-\mathrm{D}_{2} \mathrm{O}$ (9:1), $280 \mathrm{~K}$ 50-ms spin-lock mixing time assignments are show for the monomer

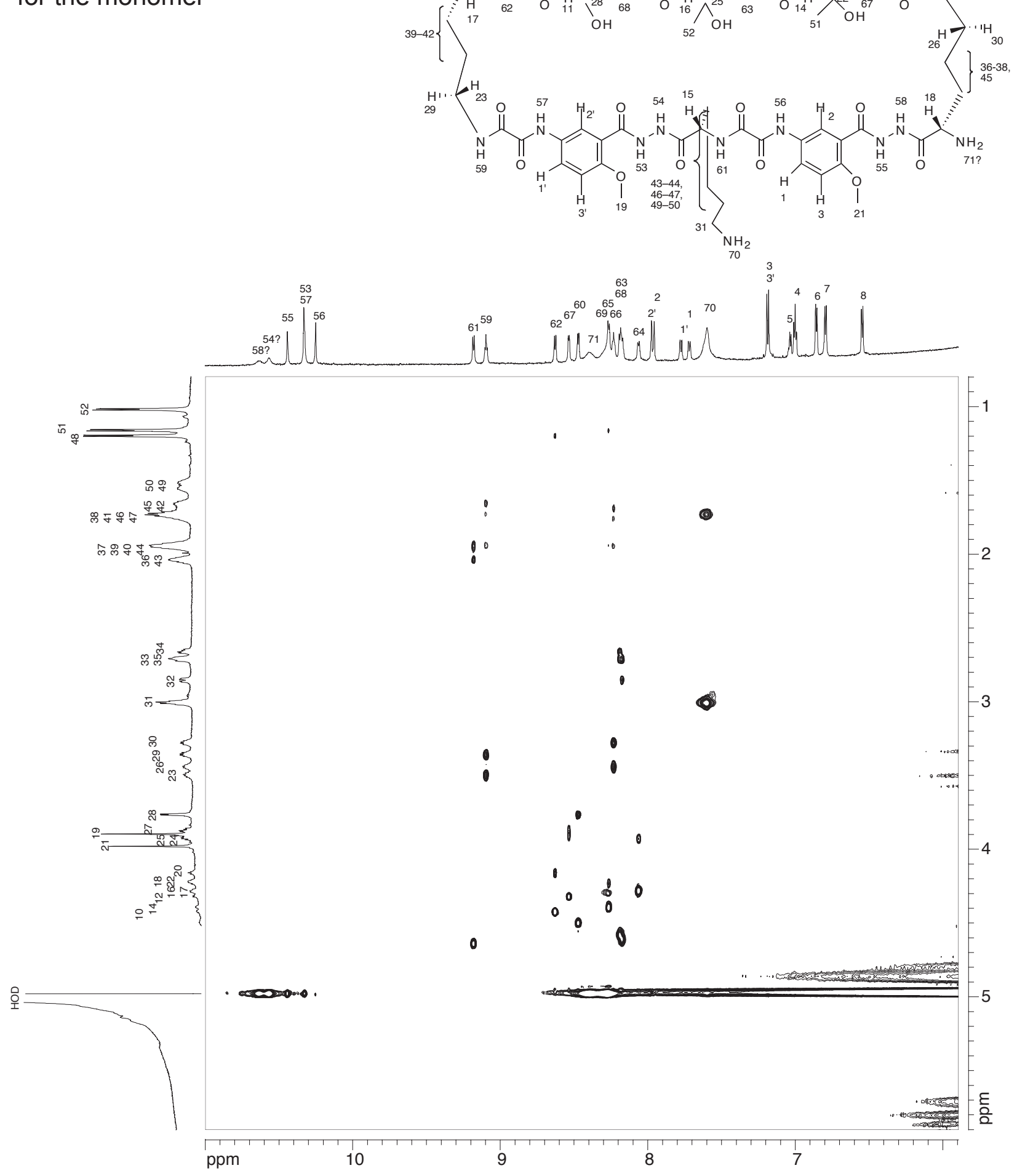


$800 \mathrm{MHz}$ NOESY spectrum of the monomer of peptide $\mathbf{3 a}$ with WATERGATE $0.6 \mathrm{mM}$ in $\mathrm{H}_{2} \mathrm{O}-\mathrm{D}_{2} \mathrm{O}(9: 1), 280 \mathrm{~K}$ 400-ms mixing time assignments are show for the monomer

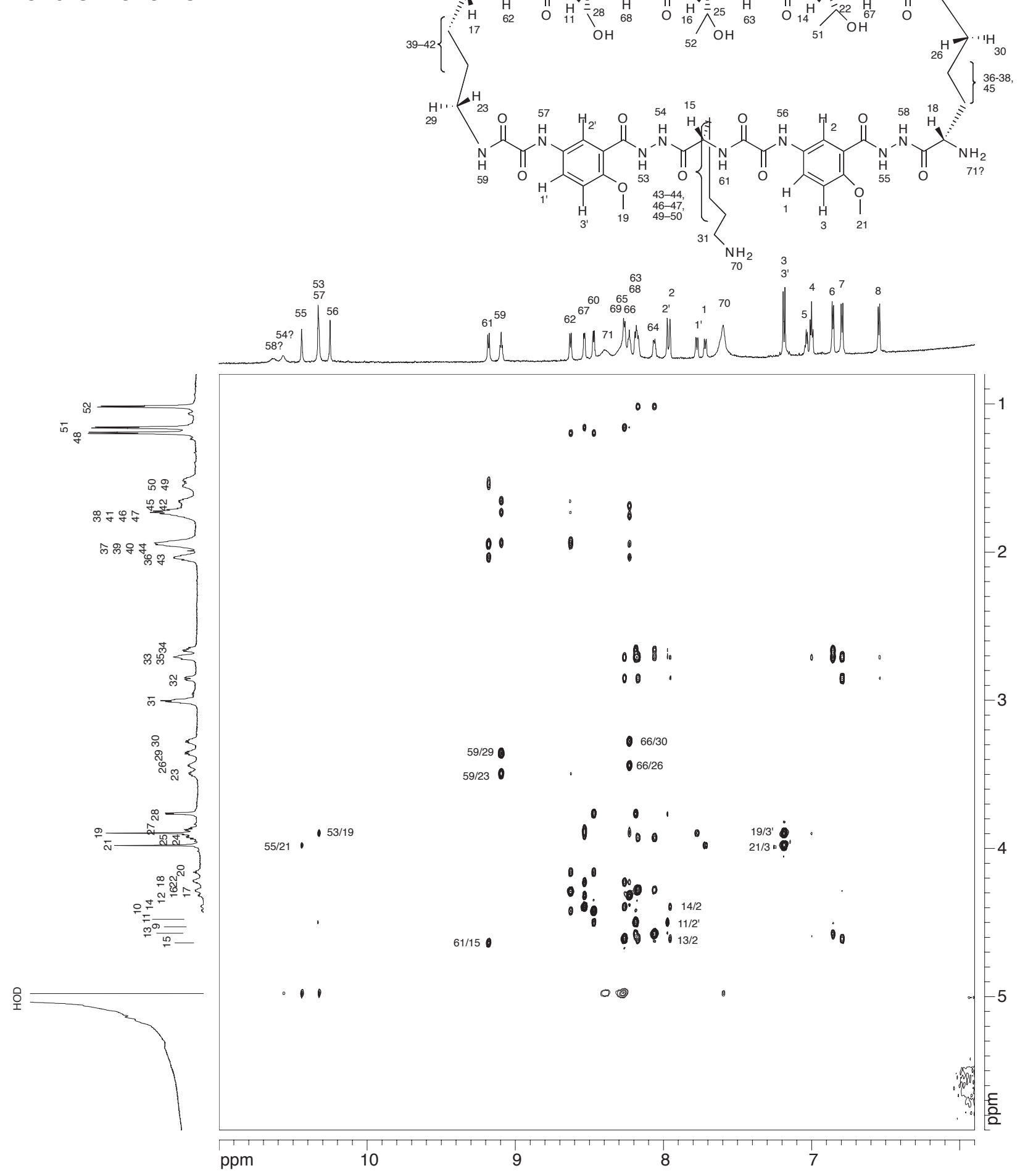


$800 \mathrm{MHz}$ NOESY spectrum of the monomer of peptide $\mathbf{3 a}$ with WATERGATE $0.6 \mathrm{mM}$ in $\mathrm{H}_{2} \mathrm{O}-\mathrm{D}_{2} \mathrm{O}$ (9:1), $280 \mathrm{~K}$ 400-ms mixing time assignments are show for the monomer

$\mathrm{NH}-\square$ protons region of a higher contour level, emphasizing intraresidue and $(\mathrm{i}, \mathrm{i}+1)$ interresidue $\mathrm{NH}-\mathrm{C}$ proton NOEs
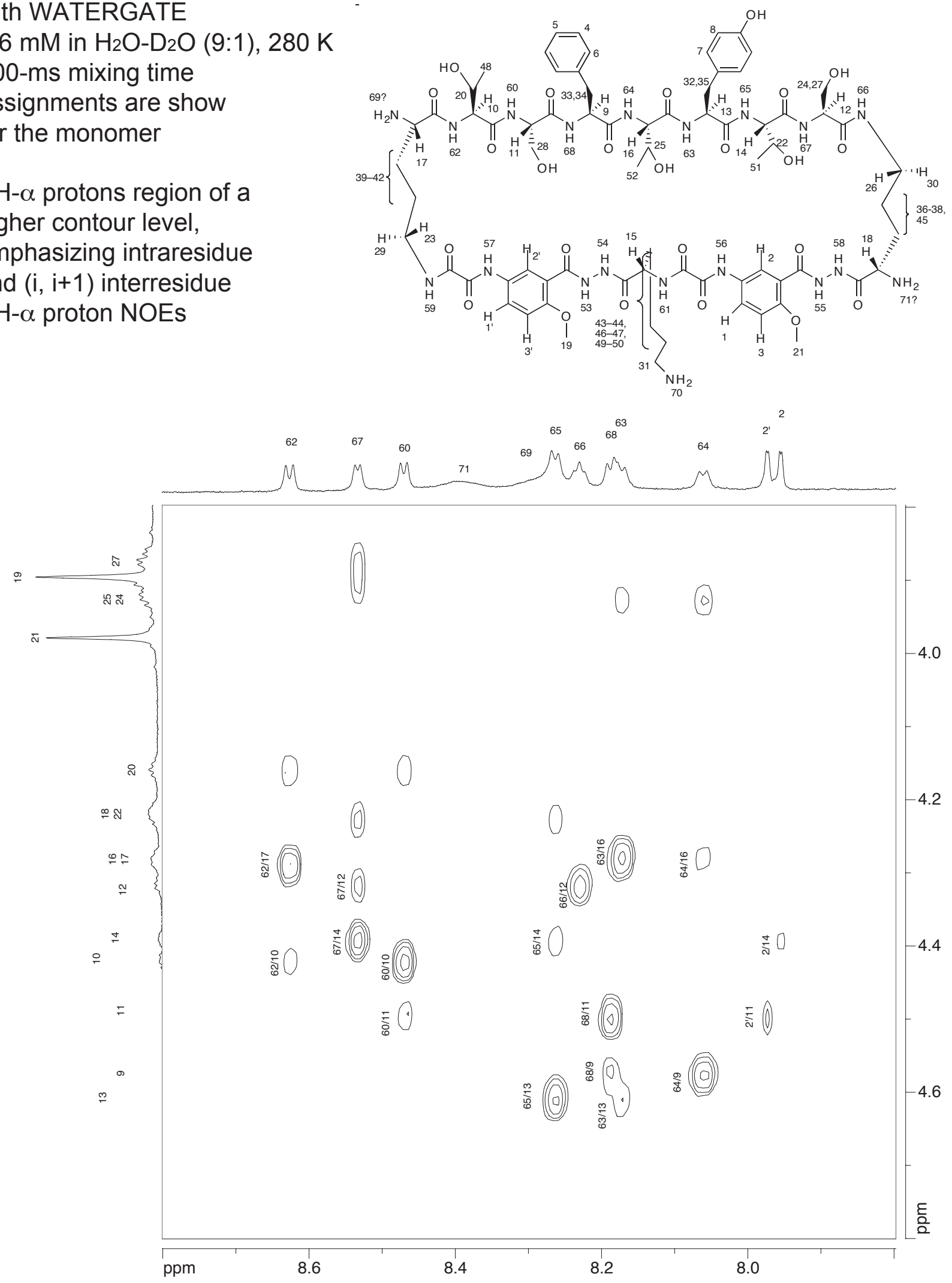
$800 \mathrm{MHz}$ NOESY spectrum of the monomer of peptide $\mathbf{3 a}$ with WATERGATE $0.6 \mathrm{mM}$ in $\mathrm{H}_{2} \mathrm{O}-\mathrm{D}_{2} \mathrm{O}(9: 1), 280 \mathrm{~K}$ 400-ms mixing time assignments are show for the monomer
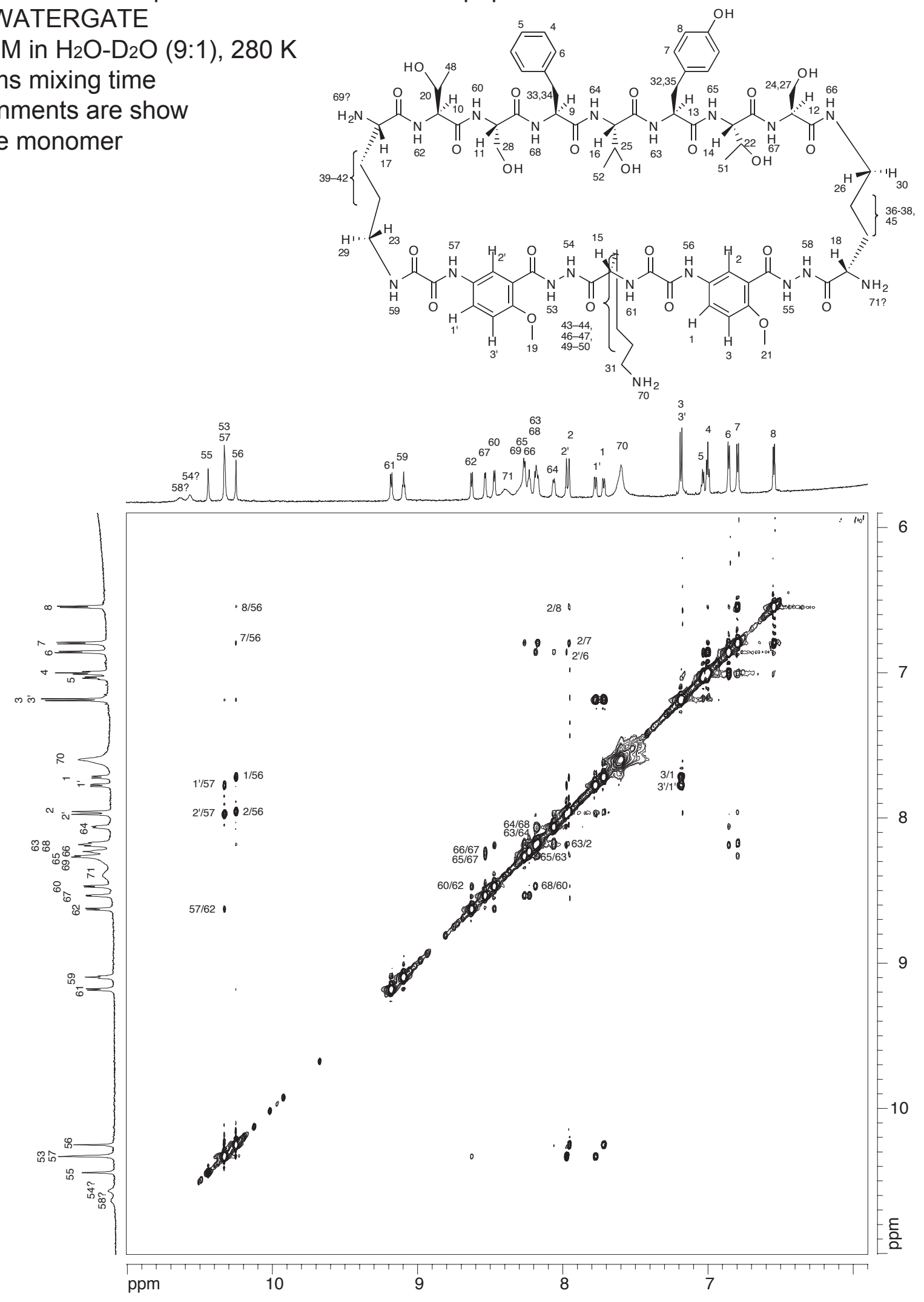
Key NOEs in the monomer of peptide $\mathbf{3 a}$

a. Interstrand NOEs

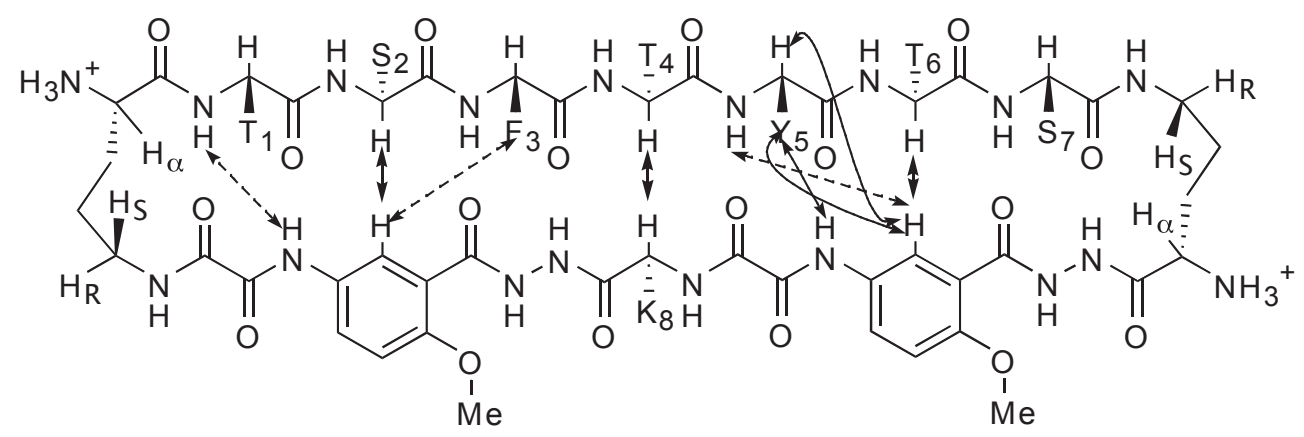

dashed arrows represent the weak or ambiguous NOEs

b. Intrastrand and turn NOEs

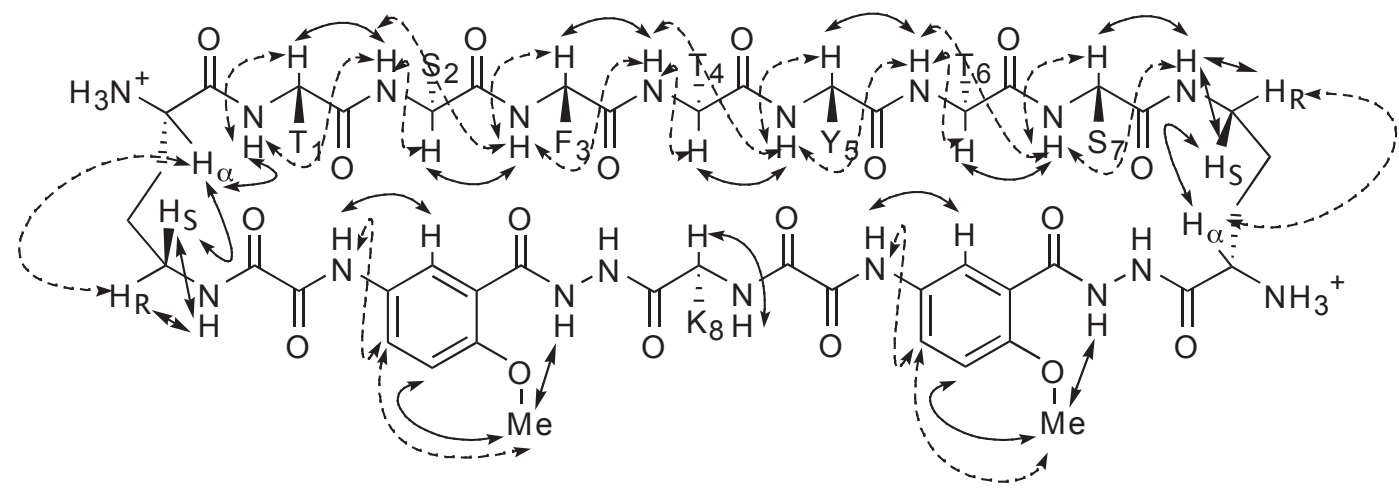

dashed arrows represent the weaker NOEs 


\section{${ }^{1} \mathrm{H}$ NMR concentration studies of peptide $3 \mathrm{a}$ in $\mathrm{D}_{2} \mathrm{O}$}

A 500- $\mu \mathrm{L}$ portion of $\mathrm{D}_{2} \mathrm{O}$ was placed in a 5-mm NMR tube, aliquots of a solution of peptide 3a (12.1 mM in $\left.\mathrm{D}_{2} \mathrm{O}\right)$ were added to the NMR tube, and a $500 \mathrm{MHz}{ }^{1} \mathrm{H}$ NMR spectrum was acquired at $280 \mathrm{~K}$ after the addition of each aliquot. Thirteen (13) aliquots of peptide 3a were added as follows: six $25-\mu \mathrm{L}$ aliquots, four $50-\mu \mathrm{L}$ aliquots, and three $100-\mu \mathrm{L}$ aliquots. The $n\left[M_{n}\right] /[M]$ ratio ( $n$ is the number of monomers in the oligomer; $\left[M_{n}\right]$ and $[M]$ are the concentrations of the oligomer and the monomer, respectively) was calculated from the ratio between the integral of the NMR resonances at 7.25-7.45 ppm (corresponding to the oligomer) and the integral of NMR resonance at 7.10-7.25 ppm (corresponding to the monomer) in each NMR spectrum. The mole fraction of oligomer, $n\left[M_{n}\right] /[M]_{t}\left(\right.$ where $\left.[M]_{t}=[M]+n\left[M_{n}\right]\right)$, was calculated from the $n\left[M_{n}\right] /[M]$ ratio and is shown in the table, below.

\begin{tabular}{|c|c|c|}
\hline$\left[\mathrm{M}_{\mathrm{t}}(\mathrm{mM})\right.$ & $\mathrm{n}\left[\mathrm{M}_{\mathrm{n}}\right] /[\mathrm{M}]$ & $\mathrm{n}\left[\mathrm{M}_{\mathrm{n}}\right] /[\mathrm{M}]_{\mathrm{t}}$ \\
\hline 0.58 & 0.0806 & 0.0746 \\
\hline 1.10 & 0.244 & 0.196 \\
\hline 1.59 & 0.587 & 0.370 \\
\hline 2.03 & 0.952 & 0.488 \\
\hline 2.44 & 1.36 & 0.576 \\
\hline 2.81 & 1.70 & 0.630 \\
\hline 3.48 & 2.38 & 0.704 \\
\hline 4.07 & 3.10 & 0.756 \\
\hline 4.57 & 3.30 & 0.767 \\
\hline 5.02 & 4.08 & 0.803 \\
\hline 5.78 & 4.80 & 0.828 \\
\hline 6.40 & 5.95 & 0.856 \\
\hline 6.90 & 6.37 & 0.864 \\
\hline
\end{tabular}


${ }^{1} \mathrm{H}$ NMR concentration studies of peptide $\mathbf{3} \mathbf{a}$ in $\mathrm{D}_{2} \mathrm{O}$

$280 \mathrm{~K}$

+ : oligomer resonances used in the integral

\# : monomer resonances used in the integral

$++\#$

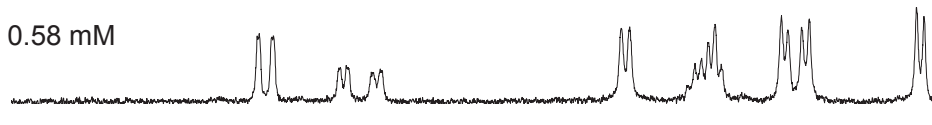

$1.10 \mathrm{mM}$

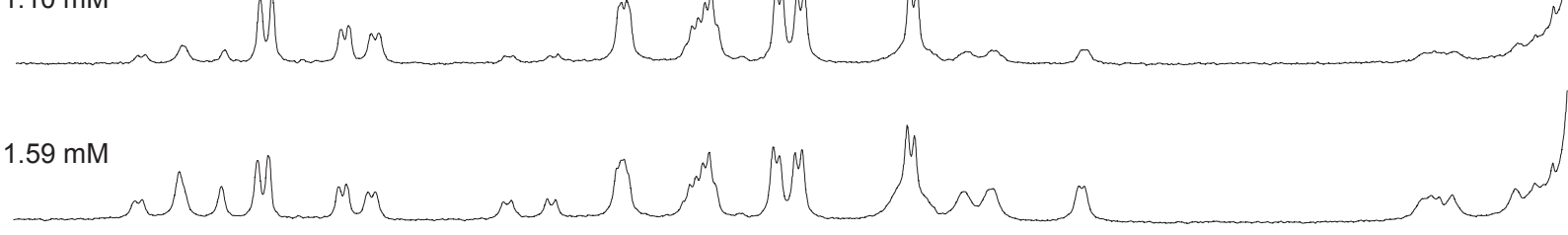

$2.03 \mathrm{mM}$

$\Lambda N M$

Mn

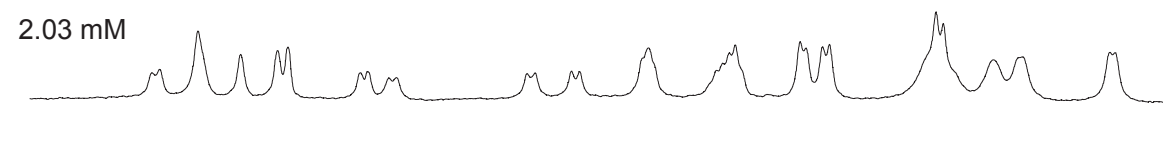

$2.44 \mathrm{mM}$

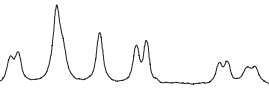

$M M M M M M M$

$2.81 \mathrm{mM}$

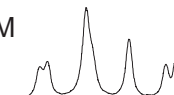

.

purction

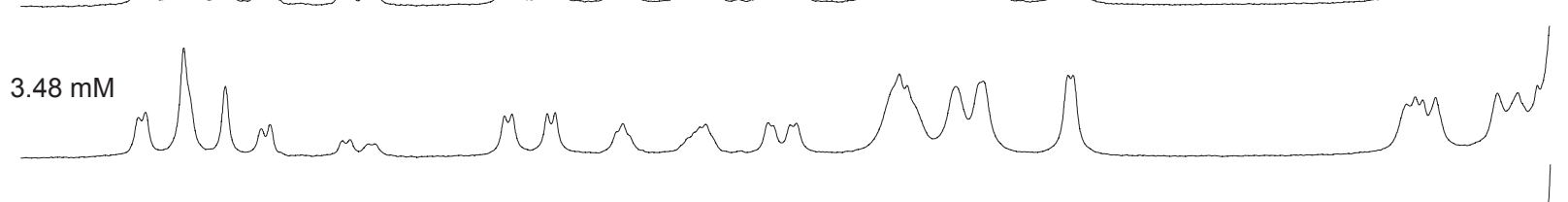

$4.07 \mathrm{mM}$

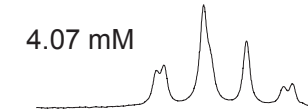

$M M \Omega M M$

$M N M$

$m m^{\prime}$

M

$M N M$

$m$

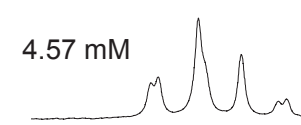

$\mu$

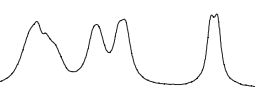

$5.02 \mathrm{~m} \mathrm{M}$

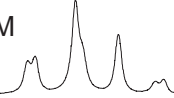

M

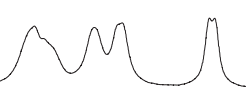

$m$

$5.78 \mathrm{mM}$
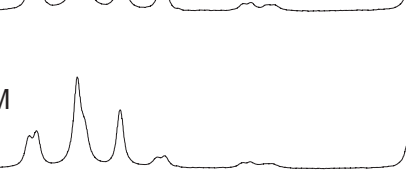

$6.40 \mathrm{mM}$

N
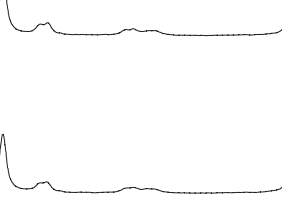

M

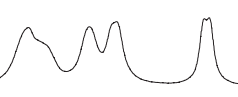

$m w$

$6.90 \mathrm{mM}$

ppm

M
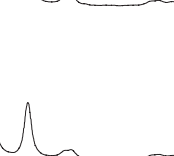

M

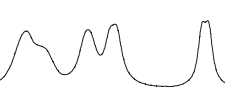

$m$

8.0

7.5

7.0
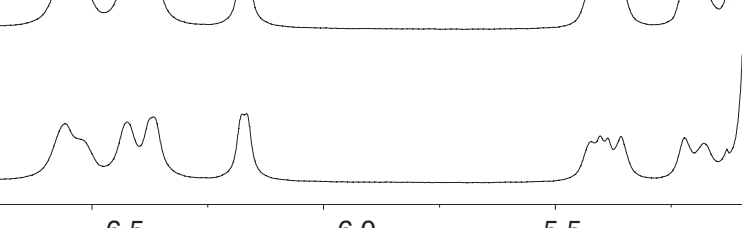


\section{Pulsed Field Gradient (PFG) NMR diffusion coefficient measurements of peptide 3a}

PFG NMR diffusion coefficient measurements were performed at $800 \mathrm{MHz}$ using a sLED pulse sequence. ${ }^{11}{ }^{1} \mathrm{H}$ NMR spectra for diffusion coefficient (D) measurements were acquired with a Varian 5-mm HCP triple-axis-gradient probe with a $60 \mathrm{G} / \mathrm{cm}$ gradient capability. The z-gradient pulse magnitude was calibrated by PFG NMR diffusion measurements of the HOD resonance in $\mathrm{D}_{2} \mathrm{O}(99.96 \%)$, which is known to have a diffusion coefficient of $1.9 \times 10^{-5}$ $\mathrm{cm}^{2} / \mathrm{s}$ at $298 \mathrm{~K} \cdot{ }^{12}$ A gradient duration $\delta=2 \mathrm{~ms}$ and a gradient delay $\Delta=50 \mathrm{~ms}$ were used for calibration.

All PFG NMR diffusion studies were performed on $0.60 \mathrm{~mL}$ samples using a gradient duration $\delta=4 \mathrm{~ms}$, a gradient delay $\Delta=100 \mathrm{~ms}$, and gradient pulse magnitudes $\mathrm{g}=0.47,9.78$, $13.64,16.61,19.10,21.30,23.29,25.12,26.82,28.42$, and $29.94 \mathrm{G} / \mathrm{cm}$. Other parameters were chosen as follows: spectral width $=12000 \mathrm{~Hz}$, acquisition time $=512 \mathrm{~ms}$, relaxation delay $=2$ sec, post-gradient eddy-current relaxation delay time $=4 \mathrm{~ms}$, and number of scans $=16$ or 32 . Data in each measurement were processed using VNMR software, and FIDs were zero-filled to $12 \mathrm{~K}$ data points and baseline-corrected. For all spectra, a $1.5-\mathrm{Hz}$ line broadening was applied, and the HOD peak was used as reference $(4.99 \mathrm{ppm}$ at $280 \mathrm{~K}, 4.92 \mathrm{ppm}$ at $285 \mathrm{~K}, 4.86 \mathrm{ppm}$ at $291 \mathrm{~K}$, and $4.78 \mathrm{ppm}$ at $298 \mathrm{~K}$ ).

Diffusion coefficients were determined by measuring the intensity (I) of different resonances as a function of $g$ and plotting $\operatorname{Ln} \mathrm{I}\left(\right.$ or $\operatorname{Ln} \mathrm{I} / \mathrm{I}_{\mathrm{o}}$ ) versus $\gamma^{2} \mathrm{~g}^{2} \delta^{2}(\Delta-\delta / 3$ ) to give a straight line with a slope equal to the negative of diffusion coefficient (D). ${ }^{13}$ (The gyromagnetic ratio for ${ }^{1} \mathrm{H}(\gamma)$ is $2.675197 \times 10^{4} \mathrm{G}^{-1} \mathrm{~s}^{-1}$.)

The diffusion coefficients of the monomer and oligomer of peptide 3a were determined as a function of total peptide concentration in $\mathrm{D}_{2} \mathrm{O}$ at $298 \mathrm{~K}$. In these studies, the intensities of the resonances at 7.8,7.2, and $6.8 \mathrm{ppm}$ were measured for the monomer and the intensities of the resonances at 7.4,6.2, and $5.4 \mathrm{ppm}$, were measured for the oligomer. Diffusion coefficients were determined for the monomer at total peptide concentrations of $0.1,0.5,1.0$, and $2.2 \mathrm{mM}$ in $\mathrm{D}_{2} \mathrm{O}$ solutions and for the oligomer at total peptide concentrations of 2.2, 3.7, 5.3, 7.6, and $10.1 \mathrm{mM}$ in $\mathrm{D}_{2} \mathrm{O}$ solutions.

11 Altieri, A. S.; Hinton, D. P.; Byrd, R. A. J. Am. Chem. Soc. 1995, 117, 7566-7567.

12 Longsworth, L. G. J. Phys. Chem. 1960, 64, 1914-1917.

13 For any species in PFG NMR diffusion measurements, signal attenuation $\left(\mathrm{I} / \mathrm{I}_{0}\right)$ is related to the translational diffusion coefficient (D) by $I / I_{o}=\exp \left[-\gamma^{2} g^{2} \delta^{2}(\Delta-\delta / 3) D\right]$. 
The diffusion coefficients of the monomer and oligomer of peptide 3a were determined as a function of temperature by measuring the diffusion coefficients of the monomer at $2 \mathrm{mM}$ total peptide concentration in $\mathrm{D}_{2} \mathrm{O}$ solution and of the oligomer at $10 \mathrm{mM}$ total peptide concentration in $\mathrm{D}_{2} \mathrm{O}$ solution. Measurements were performed at $280 \mathrm{~K}, 285 \mathrm{~K}, 291 \mathrm{~K}$, and 298 $\mathrm{K}$. In these studies, the intensity of the resonance at $7.2 \mathrm{ppm}$ was measured for the monomer and the intensities of the resonances at 7.4, 6.2, and $5.4 \mathrm{ppm}$, were measured for the oligomer. The diffusion coefficients of lysozyme, ubiquitin, and gramicidin $\mathrm{S}$ were also determined at these temperatures for comparison at the following conditions: Lysozyme (MW = 14300 Dalton, 1.4 $\mathrm{mM}$ in $\mathrm{D}_{2} \mathrm{O}$, pH adjusted to 4.7 with $\mathrm{NaOD}$ ), ubiquitin ( $\mathrm{MW}=8550$ Dalton, $1.0 \mathrm{mM}$ in $\mathrm{D}_{2} \mathrm{O}$ ), and gramicidin $\mathrm{S}\left(\mathrm{MW}=1140\right.$ Dalton, $1.1 \mathrm{mM}$ as the trifluoroacetate salt in $\left.\mathrm{D}_{2} \mathrm{O}\right)$. 
Diffusion coefficents of the monomer and oligomer of peptide 3a versus temperature $800 \mathrm{MHz}, \mathrm{D}_{2} \mathrm{O}$

$\square=4 \mathrm{~ms}, \square=100 \mathrm{~ms}$

The slope of each linear curve gives the corresponding diffusion coefficient, $D$
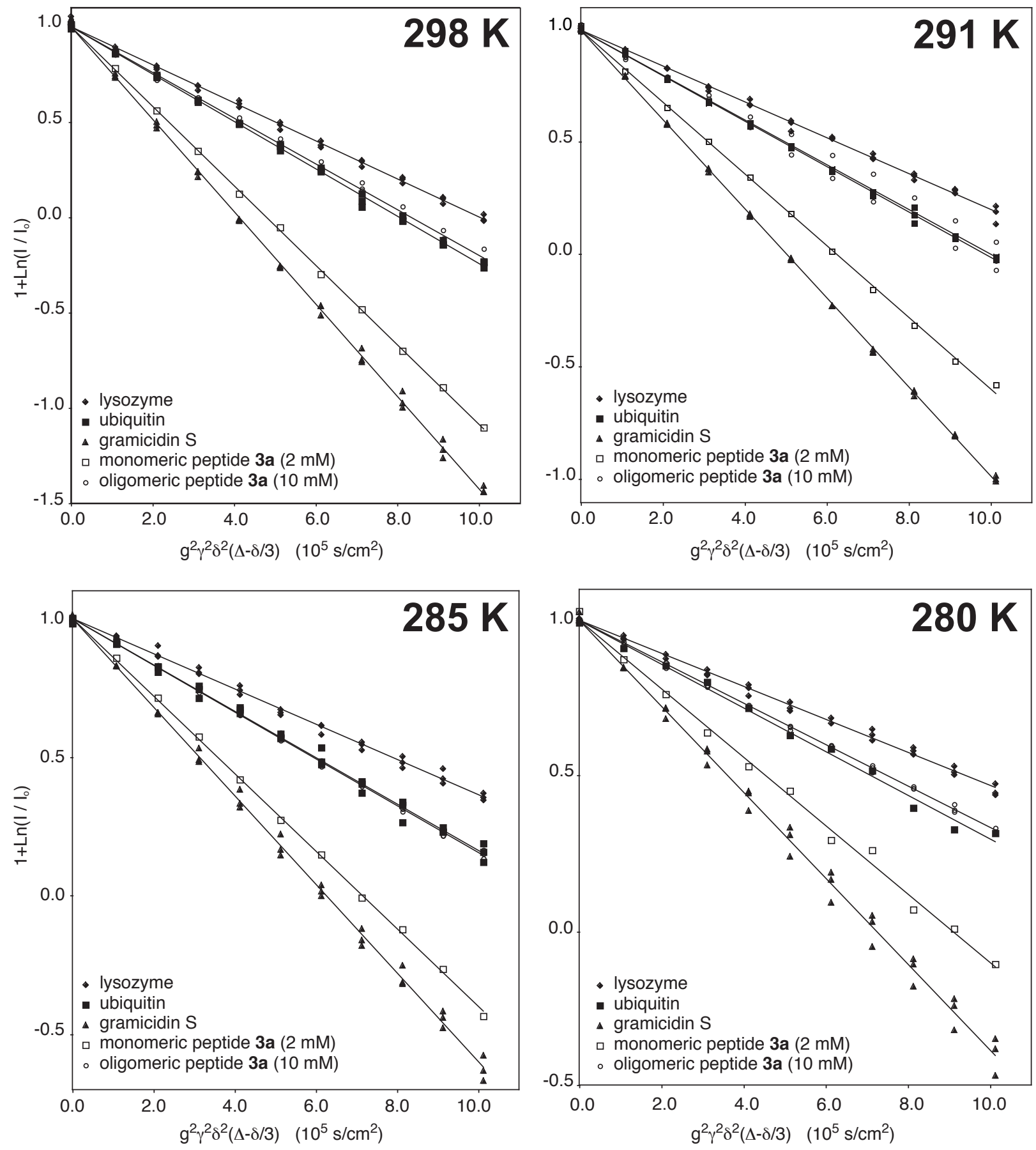
Diffusion coefficents of the monomer and oligomer of peptide 3a versus temperature $800 \mathrm{MHz}, \mathrm{D}_{2} \mathrm{O}$

Tabulated experimental diffusion coefficients

$$
\mathrm{D}\left(10^{-7} \mathrm{~cm}^{2} / \mathrm{s}\right)
$$

\begin{tabular}{|l|c|c|c|c|}
\hline comp. temp. & $\mathbf{2 9 8} \mathbf{K}$ & $\mathbf{2 9 1} \mathbf{K}$ & $\mathbf{2 8 5} \mathbf{K}$ & $\mathbf{2 8 0} \mathbf{~}$ \\
\hline Iysozyme & 10.0 & 8.0 & 6.4 & 5.3 \\
\hline ubiquitin & 12.4 & 10.1 & 8.4 & 7.1 \\
\hline gramicidin S & 24.2 & 19.8 & 16.0 & 13.9 \\
\hline monomer of peptide 3a (2 $\mathbf{~ M M )}$ & 20.9 & 16.0 & 14.0 & 11.1 \\
\hline oligomer of peptide 3a (10 $\mathbf{~ M M )}$ & 12.0 & 10.0 & 8.4 & 6.7 \\
\hline
\end{tabular}

The ratio of the diffusion coefficients of the oligomer and the monomer in different temperature

\begin{tabular}{|c|l|l|l|l|}
\hline Doligomer $/ D_{\text {monomer }}$ & 0.57 & 0.62 & 0.60 & 0.60 \\
\hline
\end{tabular}




\section{Analytical ultracentrifugation studies of peptide 3a}

All sedimentation experiments were performed with a Beckman Optima XL-I at the Center for Analytical Ultracentrifugation of Macromolecular Assemblies at the University of Texas Health Science Center at San Antonio. Sedimentation equilibrium and velocity, and Monte Carlo analyses were performed with UltraScan ${ }^{14}$ version 7.2. Hydrodynamic corrections for buffer conditions were made according to data published by Laue et $a l .{ }^{15}$ and as implemented in UltraScan.

Fringe units were converted to molar concentration by comparing sedimentation velocity experiments performed with the UV absorption optics to velocity experiments performed with the Rayleigh interference optics. A sedimentation velocity experiment of a 10 -fold dilution of a solution of peptide 3a was performed at 60,000 rpm and scanned in absorbance mode at $280 \mathrm{~nm}$ (data not shown). In the same experiment, the undiluted solution was measured by using Rayleigh interference optics. Both velocity experiments were fitted to finite element solutions of the Lamm equation, ${ }^{16}$ which reported reliable initial concentrations for both the absorbance the interference experiments. Distribution analysis was performed with the enhanced van HoldeWeischet analysis. ${ }^{17}$ After correcting for path-length, the molar concentration of the diluted and undiluted peptide 3a solutions were calculated based on the molar extinction coefficient of $20,980 \mathrm{OD}_{280} \mathrm{~mol}^{-1} \mathrm{~cm}^{-1}$ of peptide 3a. The molar extinction coefficient at $280 \mathrm{~nm}$ was determined from the partial amino acid contributions according to the method by Gill and von Hippel ${ }^{18}$ and based on the measured molar absorptivity of $\mathrm{Hao}$ as $9850 \mathrm{OD}_{280} \mathrm{~mol}^{-1} \mathrm{~cm}^{-1}$. ${ }^{19}$ Based on this approach, the molar refractive index in the machine for peptide 3a was determined as 6169.4 fringes $\mathrm{mol}^{-1} \mathrm{~cm}^{-1}$.

\footnotetext{
14 (a) Demeler, B. UltraScan 7.2 - An integrated data analysis software package for sedimentation experiments; University of Texas Health Science Center: San Antonio, Dept. of Biochemistry, 2005. (b) http://www.ultrascan.uthscsa.edu.

15 Laue, T. M.; Shah, B. D.; Ridgeway, T. M.; Pelletier, S. L. In: Analytical Ultracentrifugation in Biochemistry and Polymer Science; Harding, S. E., Rowe, A. J., Horton, J. C., Eds.; Royal Society of Chemistry: Cambridge, UK, 1992; pp 90-125.

16 Demeler, B.; Saber, H. Biophys. J. 1998, 74, 444-454.

17 Cao W, Demeler B. Biophys. J. 2005, 89, 1589-602.

18 Gill, S. C., Von Hippel, P. H. Anal. Biochem. 1989, 182, 319-326.

19 The molar absorptivity of Hao unit at $280 \mathrm{~nm}$ was determined by measuring the slope of the linear plots of UVabsorbance versus concentration for $i$-Pr-Hao-NHMe solutions in water-ethanol $(9: 1)$.
} 
For equilibrium sedimentation experiments, the partial specific volume of peptide $\mathbf{3 a}$ was estimated to be $0.706 \mathrm{~cm}^{3} / \mathrm{g}$ from peptide sequence according to the method by Durchschlag, ${ }^{20,21}$ and as implemented in UltraScan. Multiple models were fitted to all data. The most appropriate model was chosen based on visual inspection of the residual run patterns, and based on the best statistics. 95\% confidence intervals were determined by Monte Carlo analysis. Three loading concentrations of peptide 3a covering a broad concentration range (15 $\mu \mathrm{M}, 1.5 \mathrm{mM}$, and $9 \mathrm{mM})$ were measured in $\mathrm{H}_{2} \mathrm{O}$ containing $100 \mathrm{mM} \mathrm{NaCl}$ and at $20^{\circ} \mathrm{C}$. Absorbance scans $(15 \mu \mathrm{M}, 230$ $\mathrm{nm})$ and Rayleigh interference scans $(1.5 \mathrm{mM}$ and $9 \mathrm{mM})$ were collected at equilibrium from three speeds (50 000, 55 000, and $60000 \mathrm{rpm})$. Absorbance samples were spun in 2-channel titanium centerpieces (Nanolytics, Gesellschaft für Kolloidanalytik mbH Hauptstr. 20, 14624 Dallgow, Berlin, Germany) in the AN-60-TI rotor.

20 (a) Durchschlag, H. In: Thermodynamic Data for Biochemistry and Biotechnology; Hinz, H.-J., Ed.; SpringerVerlag: New York, 1986; pp 45-128. (b) Durchschlag, H.; Zipper, P. Progr. Colloid Polym. Sci. 1994, 94, 20 39.

21 The Hao subunit was calculated to have a MW of 235.12, a volume of $152.8 \mathrm{~cm}^{3} / \mathrm{mol}$, and a partial specific volume of $0.65 \mathrm{~cm}^{3} / \mathrm{g}$ according to reference $20 \mathrm{~b}$. 


\section{Weight-average measurements of each mode in a bimodal distribution.}

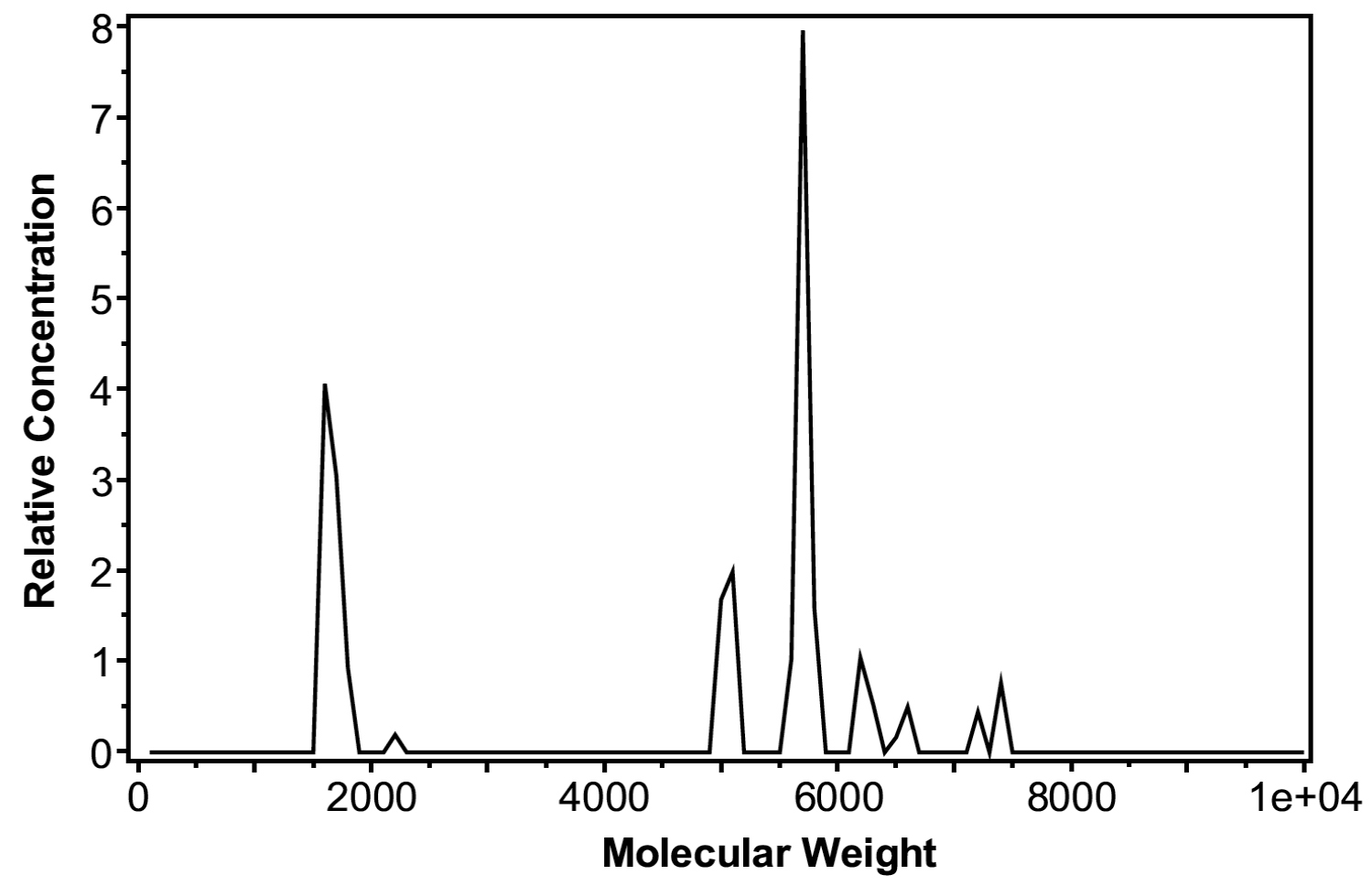

Figure. Molecular weight distribution from a global fit to a combination of equilibrium data from Rayleigh interference and UV absorption experiments. The molecular weight distribution ranges between 100-10,000 Dalton, and has a grid spacing of 100 Dalton. Model functions for each molecular weight were calculated and the amplitudes were fitted to the data using non-negatively constrained linear least squares. ${ }^{22}$

22 Lawson, C. L.; Hanson, R. J. In: Solving Least Squares Problems; Prentice-Hall, Inc.: Englewood Cliffs, New Jersey, 1974. 


\section{${ }^{1}$ H NMR studies of control peptides 6}

Peptide 6 was studied by $500 \mathrm{MHz}$ TOCSY and Tr-ROESY experiments at $280 \mathrm{~K}$ on an $8.0 \mathrm{mM} \mathrm{D}_{2} \mathrm{O}$ solution of the peptide. Spin-lock mixing times of $400 \mathrm{~ms}$ and $150 \mathrm{~ms}$ were used for Tr-ROESY and TOCSY experiments, respectively. All 2D spectra were acquired with 2048 data points in the $f_{2}$ domain and 512 data points in the $f_{1}$ domain and were processed by zerofilling to a final matrix of $1024 \times 1024$ real points. 
1D 1H NMR of control peptide 6

$500 \mathrm{MHz}$

$8.0 \mathrm{mM}$ in $\mathrm{D}_{2} \mathrm{O}, 280 \mathrm{~K}$

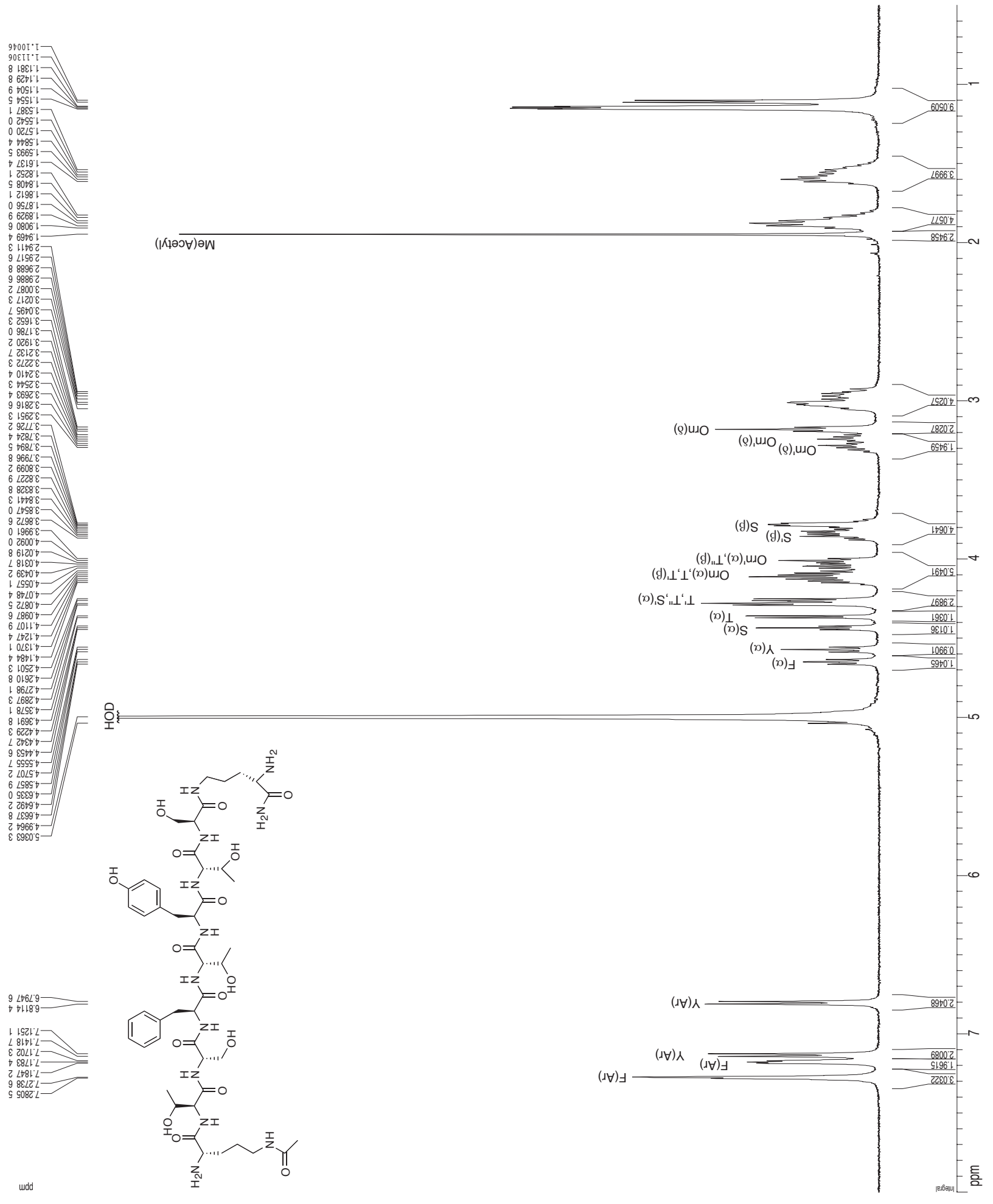


TOCSY spectrum of control peptide 6

$500 \mathrm{MHz}$ with water suppression (100-ms mixing time)

$8.0 \mathrm{mM}$ in $\mathrm{D}_{2} \mathrm{O}, 280 \mathrm{~K}$
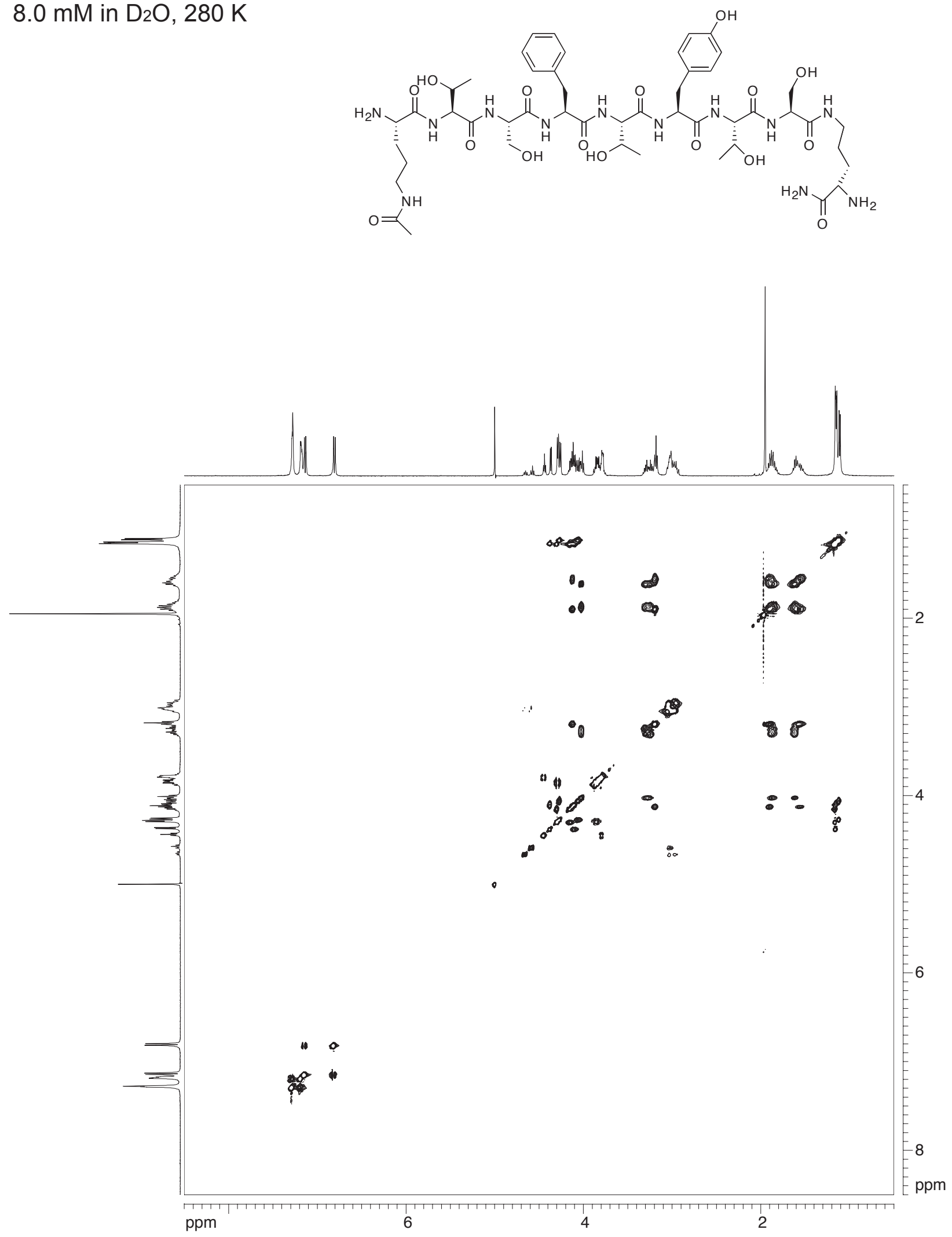
ROESY spectrum of control peptide 6

$500 \mathrm{MHz}$ with water suppression (400-ms mixing time)

$8.0 \mathrm{mM}$ in $\mathrm{D}_{2} \mathrm{O}, 280 \mathrm{~K}$
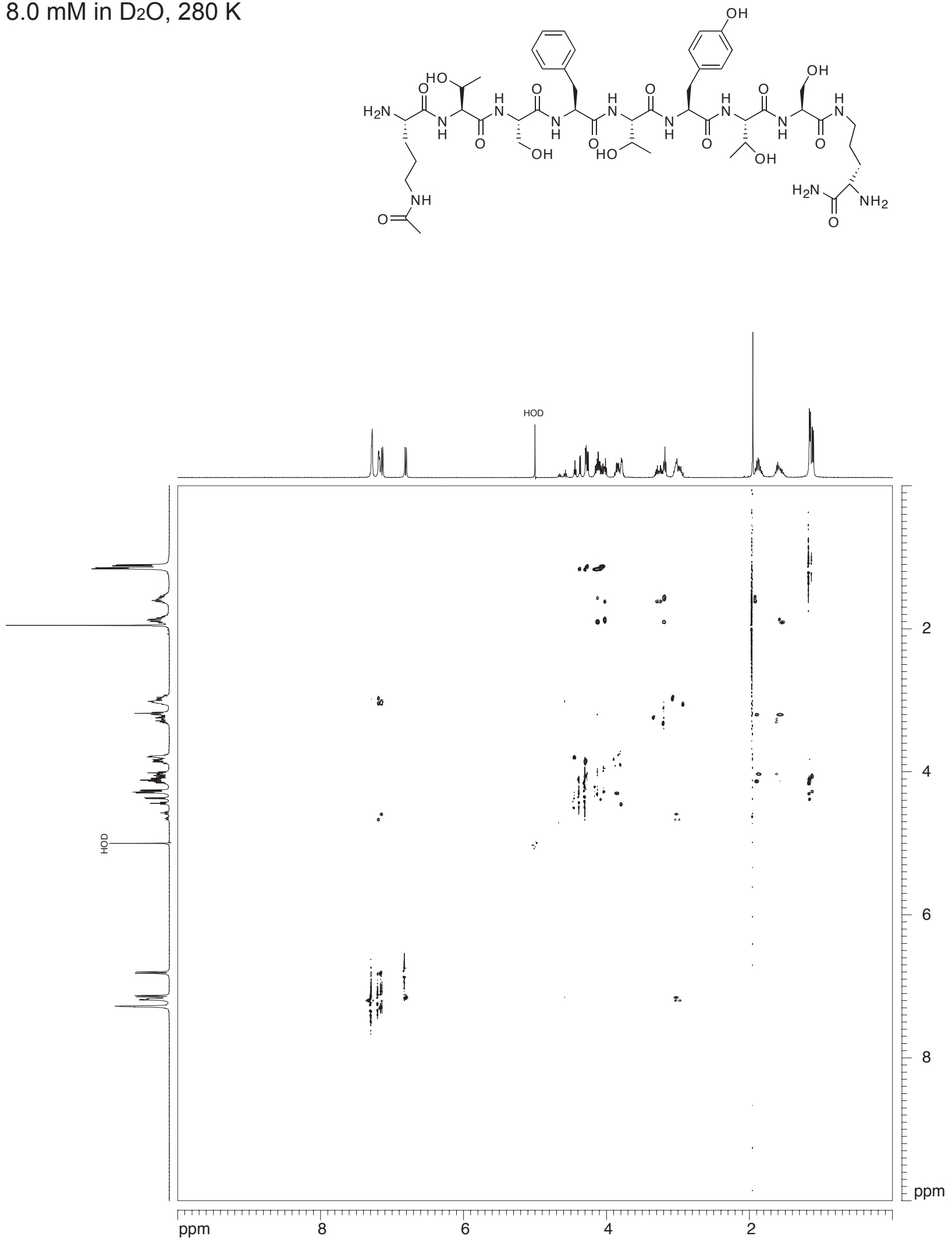


\section{${ }^{1} \mathrm{H}$ NMR studies of peptides $3 \mathrm{a}-3 \mathrm{n}$}

Solutions of peptides 3a-3n were prepared gravimetrically by dissolving an appropriate weight of the peptides in an appropriate volume of solvent. In calculating molecular weights, all amino groups were assumed to be protonated as TFA salts.

The ${ }^{1} \mathrm{H}$ NMR resonances of the monomers of peptides $\mathbf{3 a}-\mathbf{3 n}$ were assigned by $500 \mathrm{MHz}$ TOCSY and Tr-ROESY experiments at $298 \mathrm{~K}$, using water presaturation as needed, on 0.4-1.2 $\mathrm{mM} \mathrm{D} \mathrm{D}_{2} \mathrm{O}$ and $\mathrm{H}_{2} \mathrm{O}-\mathrm{D}_{2} \mathrm{O}$ (90:10) solutions of the peptides. Spin-lock mixing times of $300 \mathrm{~ms}$ and $150 \mathrm{~ms}$ were used for Tr-ROESY and TOCSY experiments, respectively. All 2D spectra were acquired with 2048 data points in the $f_{2}$ domain and 512 data points in the $f_{I}$ domain and were processed by zero-filling to a final matrix of 1024 x 1024 real points.

The self-association properties of the peptides in $\mathrm{D}_{2} \mathrm{O}$ were studied by comparing the ${ }^{1} \mathrm{H}$ NMR spectra of the dilute samples $(0.4-1.2 \mathrm{mM})$ to those of concentrated samples $(5-16 \mathrm{mM})$ at $298 \mathrm{~K}$. In cases in which no differences were observed at $298 \mathrm{~K}$, the experiments were repeated at lower temperatures (e.g., $280 \mathrm{~K}$ ). 
1D ${ }^{1} \mathrm{H}$ NMR spectrum of the monomer of peptide $3 \mathrm{a}$

$500 \mathrm{MHz}$

$1 \mathrm{mM}$ in $\mathrm{D}_{2} \mathrm{O}, 298 \mathrm{~K}$

01000
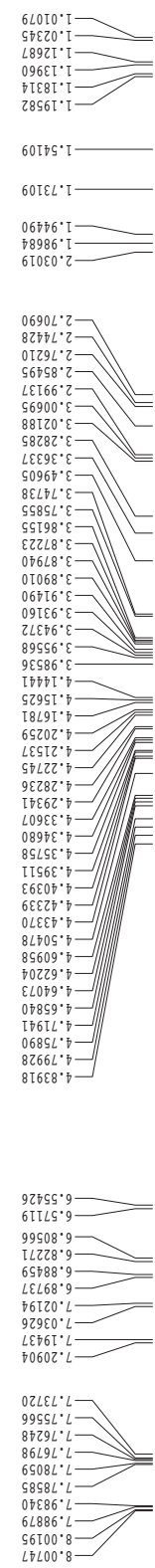

I
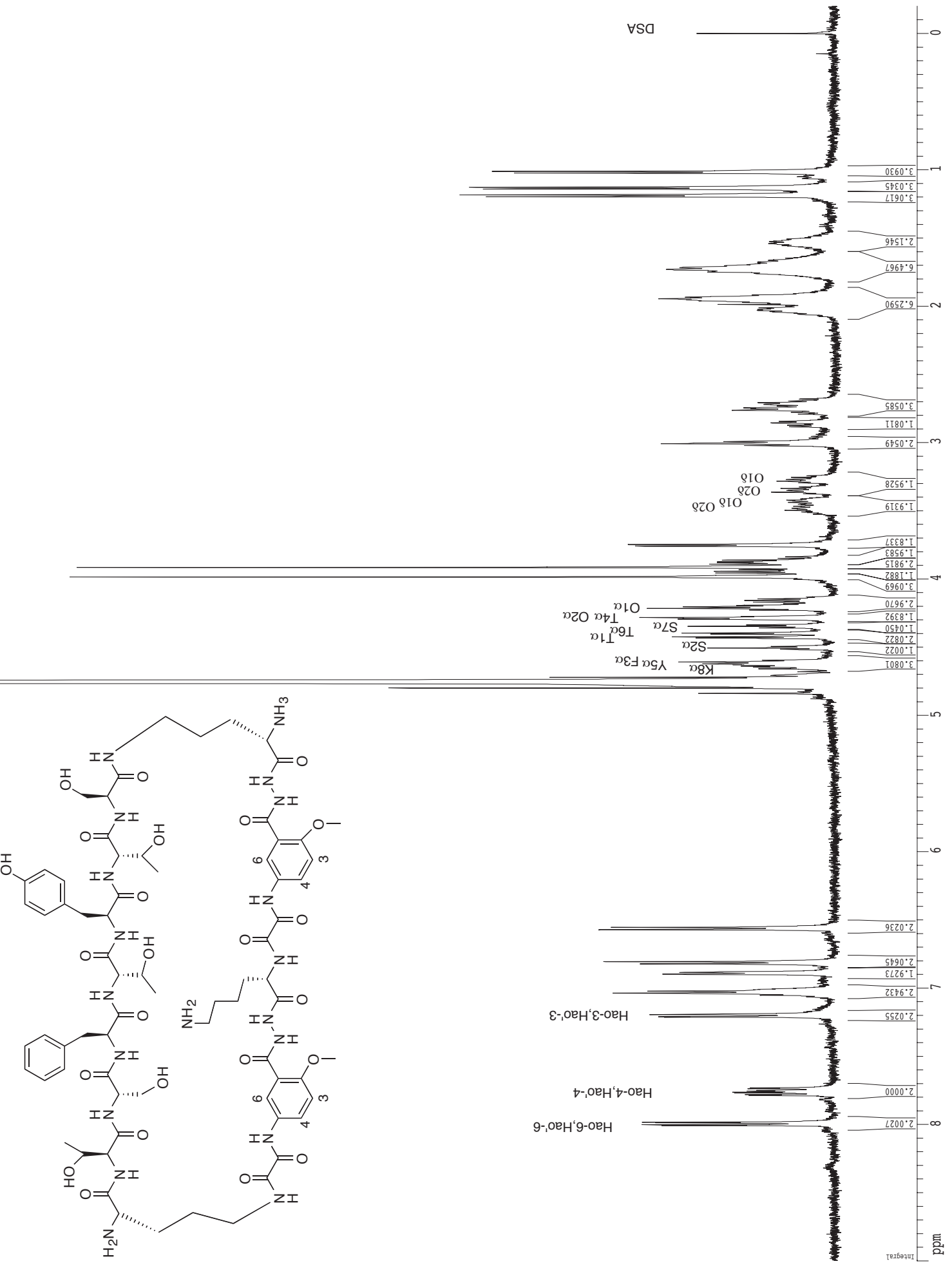
1D ${ }^{1} \mathrm{H}$ NMR spectrum of the monomer of peptide $3 \mathbf{b}$

$500 \mathrm{MHz}$

$1 \mathrm{mM}$ in $\mathrm{D}_{2} \mathrm{O}, 298 \mathrm{~K}$
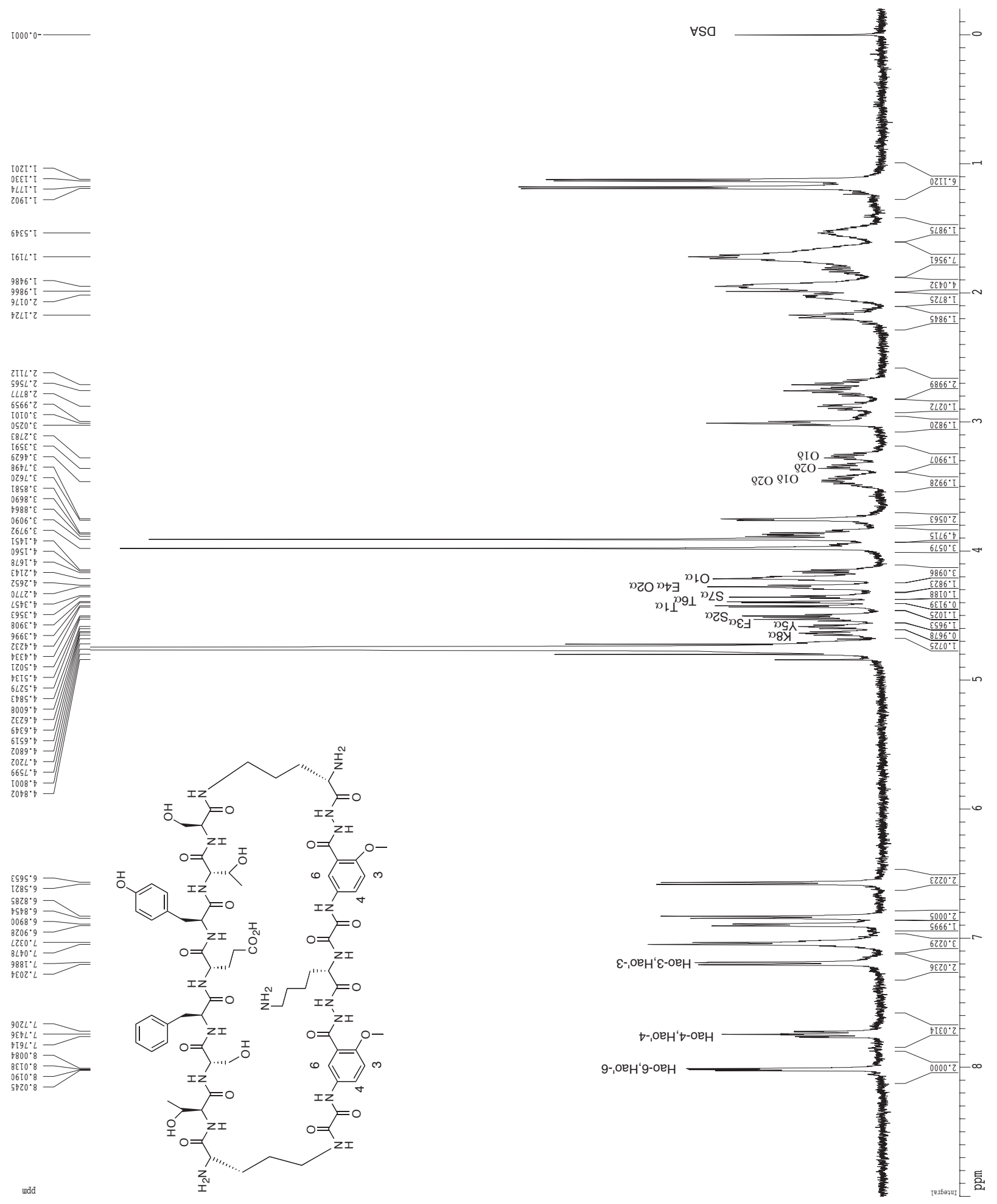

udd . 
TOCSY spectrum of the monomer of peptide $\mathbf{3 b}$ $500 \mathrm{MHz}$ (150-ms mixing time)

$1.0 \mathrm{mM}$ in $\mathrm{D}_{2} \mathrm{O}, 298 \mathrm{~K}$

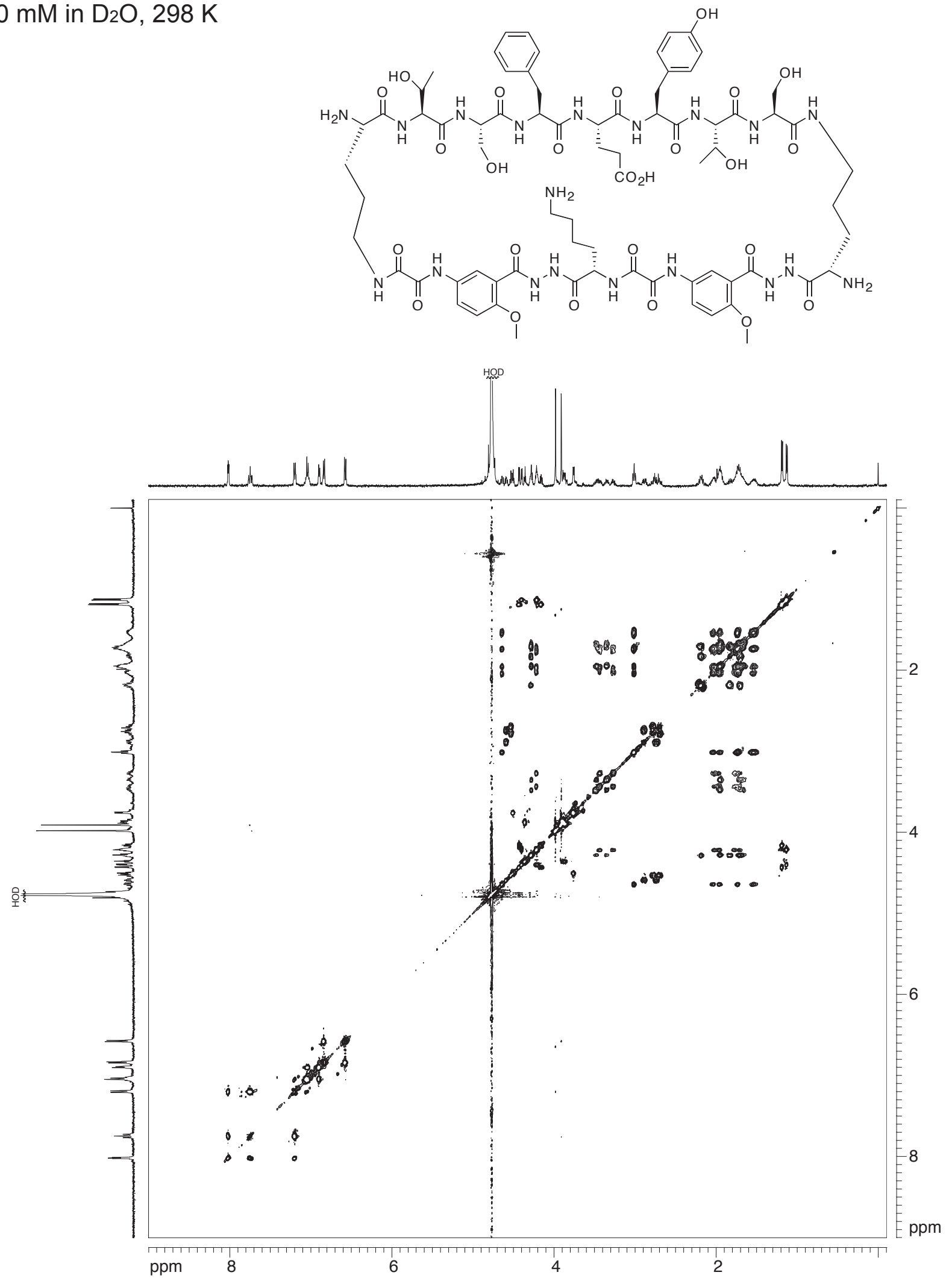


ROESY spectrum of the monomer of peptide $\mathbf{3 b}$ $500 \mathrm{MHz}$ (300-ms mixing time)

\section{$1.0 \mathrm{mM}$ in $\mathrm{D}_{2} \mathrm{O}, 298 \mathrm{~K}$}

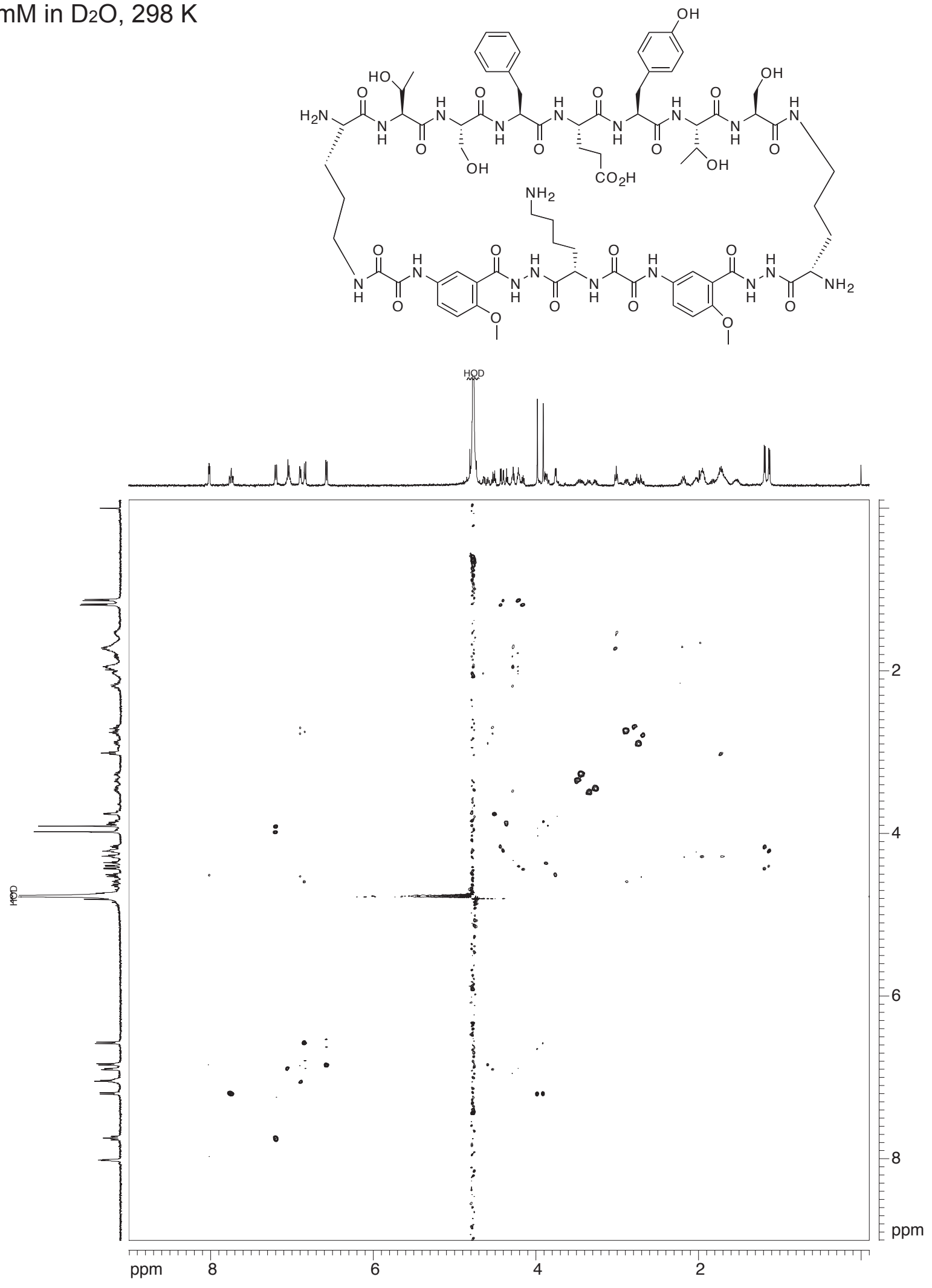


Overlay of $1 \mathrm{D}{ }^{1} \mathrm{H}$ NMR spectra of dilute and concentrated solutions of peptide $3 \mathbf{b}$ $500 \mathrm{MHz}$

$\mathrm{D}_{2} \mathrm{O}, 298 \mathrm{~K}$

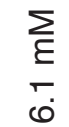

롱

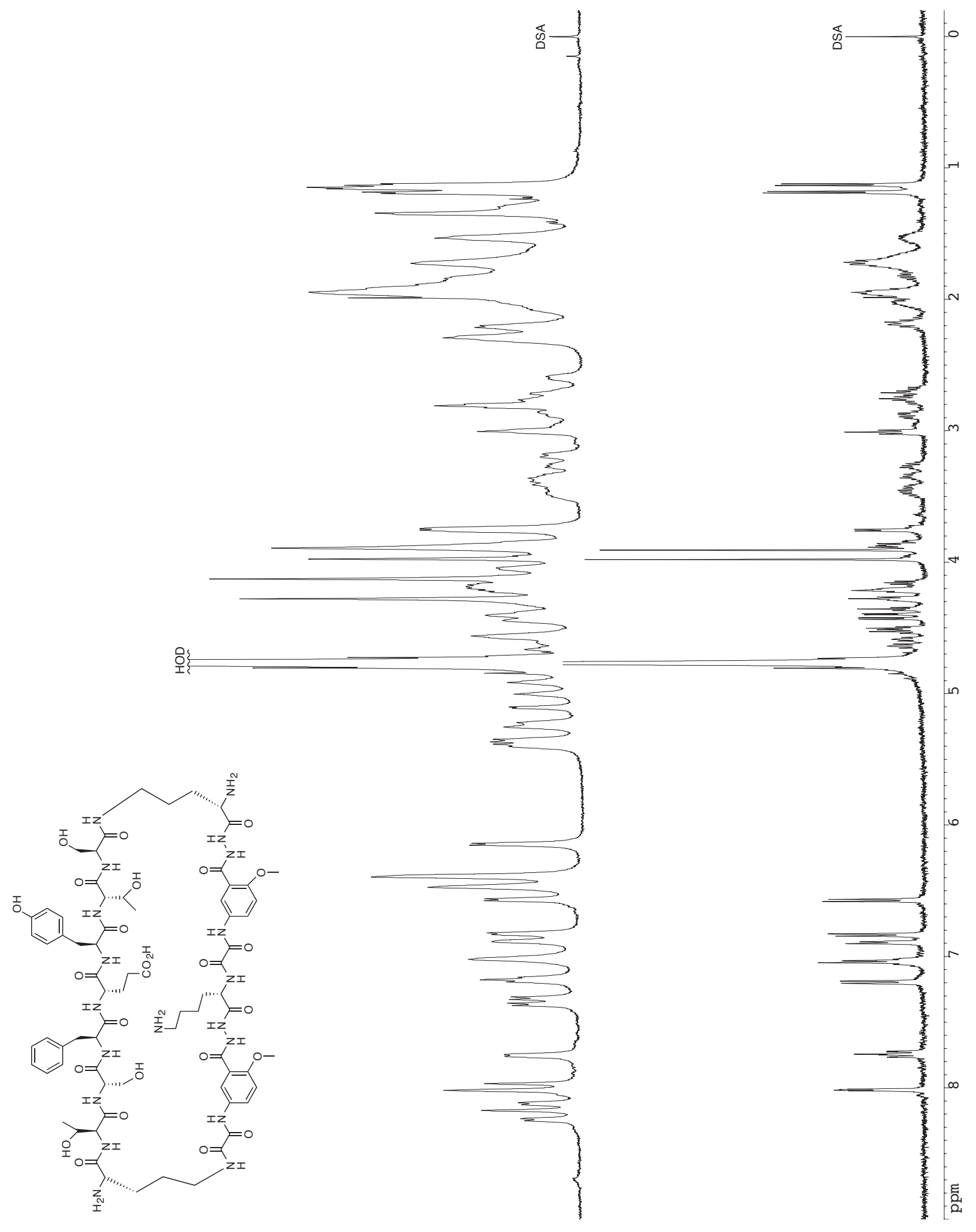


1D ${ }^{1} \mathrm{H}$ NMR spectrum of the monomer of peptide $3 \mathrm{c}$

$500 \mathrm{MHz}$

$1 \mathrm{mM}$ in $\mathrm{D}_{2} \mathrm{O}, 298 \mathrm{~K}$
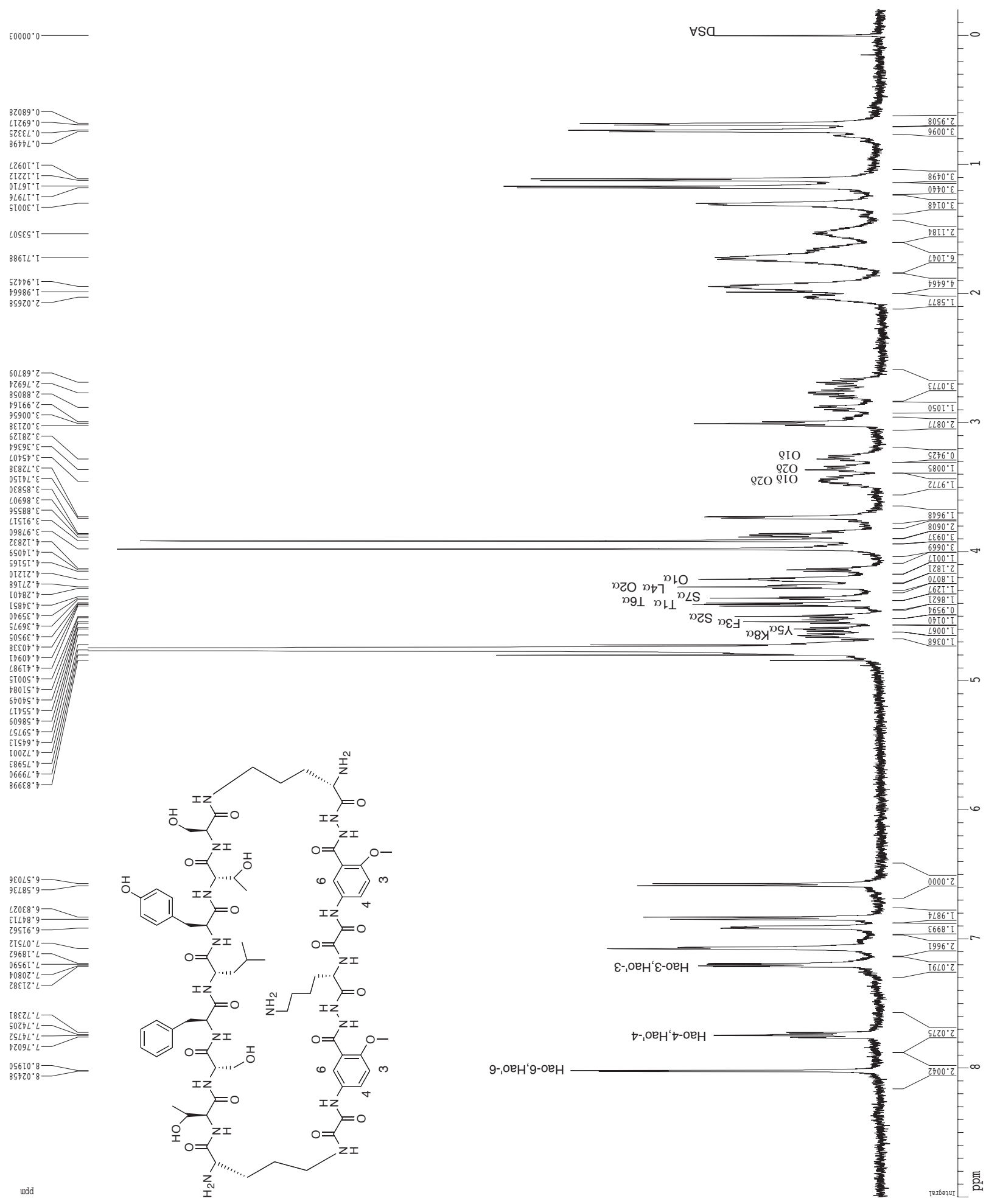
TOCSY spectrum of the monomer of peptide $3 \mathrm{c}$ $500 \mathrm{MHz}$ (150-ms mixing time)

$1.0 \mathrm{mM}$ in $\mathrm{D}_{2} \mathrm{O}, 298 \mathrm{~K}$

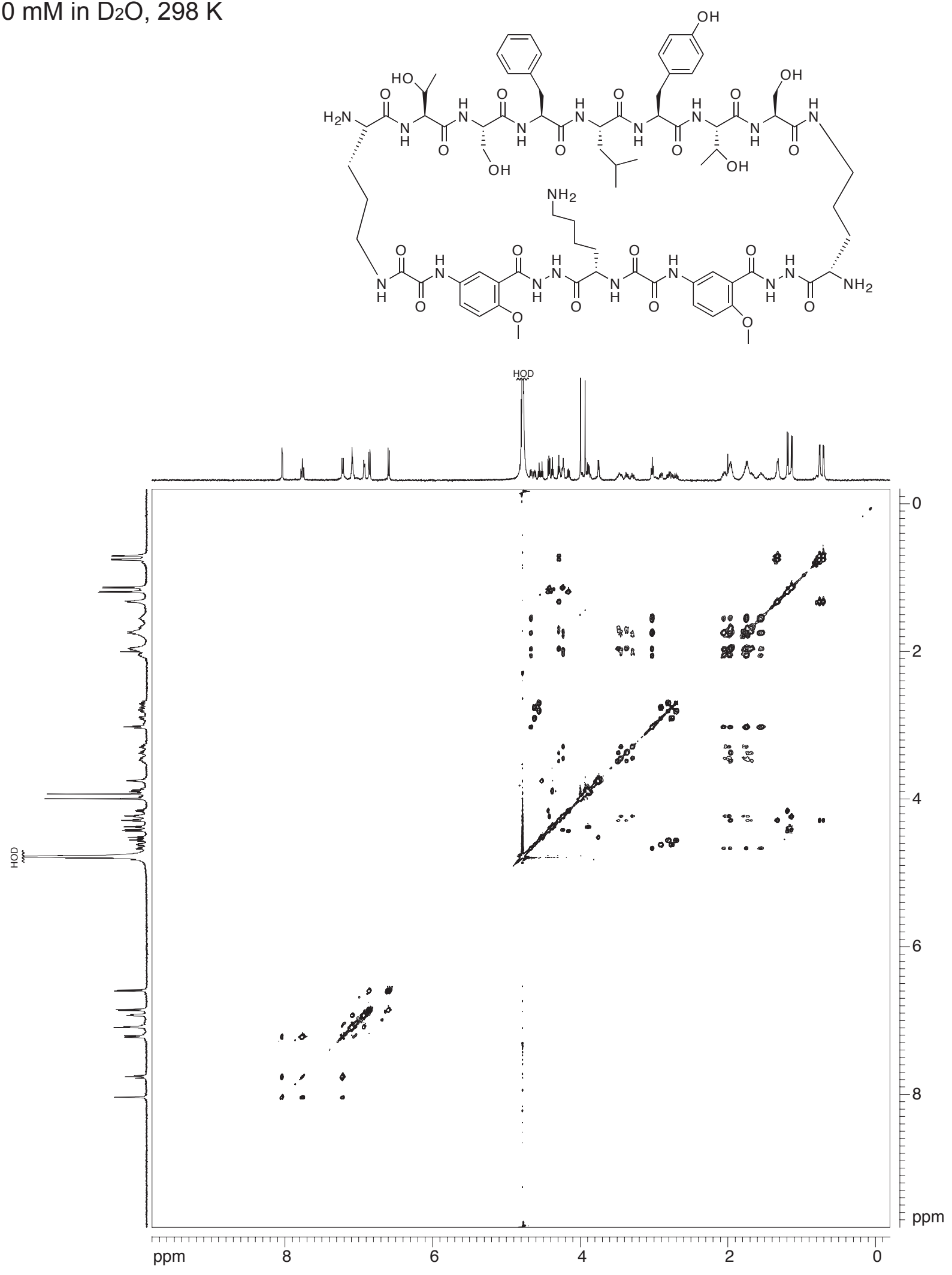


ROESY spectrum of the monomer of peptide $3 \mathrm{c}$ $500 \mathrm{MHz}$ (300-ms mixing time)

$1.0 \mathrm{mM}$ in $\mathrm{D}_{2} \mathrm{O}, 298 \mathrm{~K}$

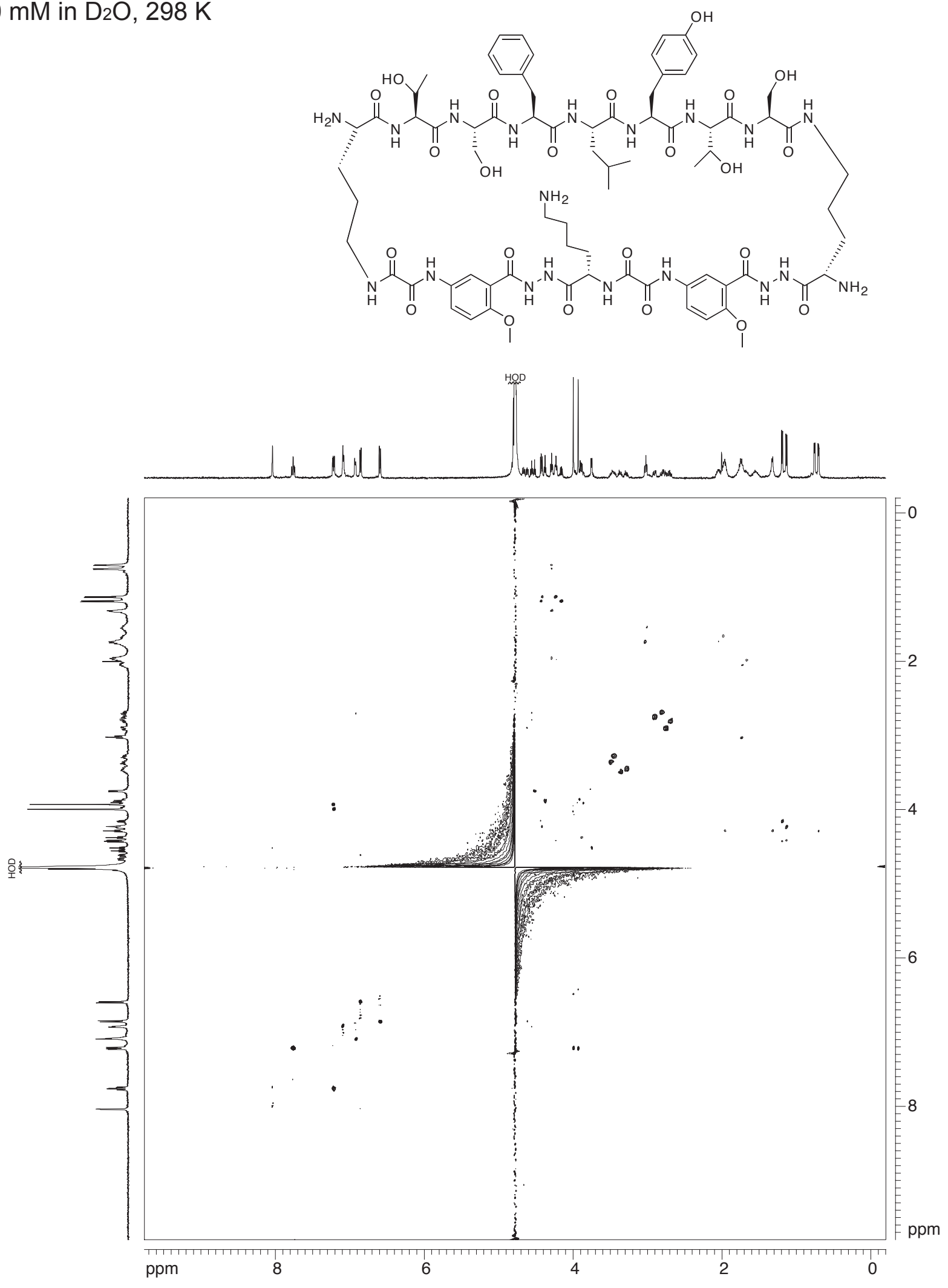


Overlay of $1 \mathrm{D}{ }^{1} \mathrm{H}$ NMR spectra of dilute and concentrated solutions of peptide $3 \mathrm{c}$ $500 \mathrm{MHz}$

$\mathrm{D}_{2} \mathrm{O}, 298 \mathrm{~K}$
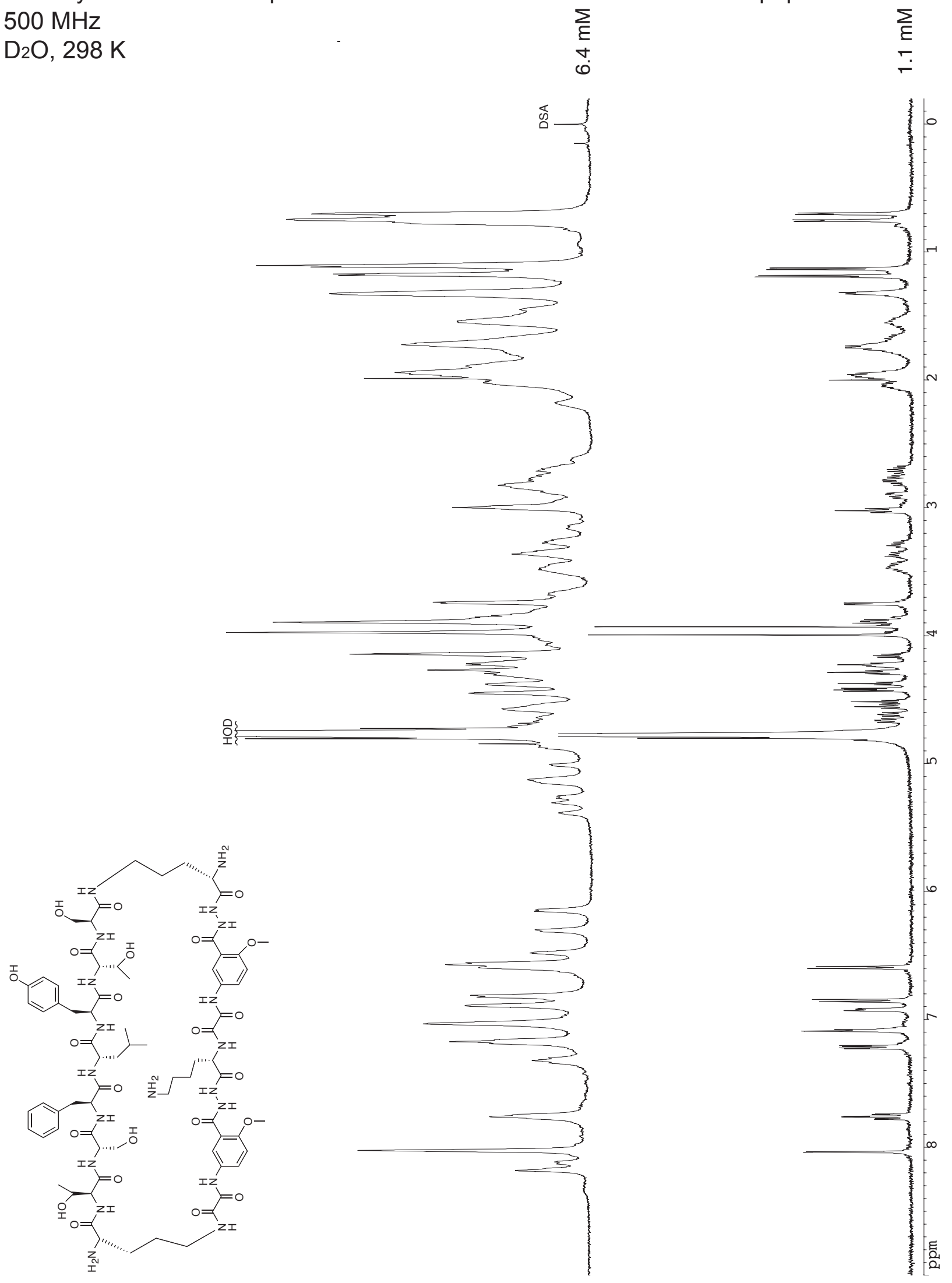
1D ${ }^{1} \mathrm{H}$ NMR spectrum of the monomer of peptide $3 \mathrm{~d}$

$500 \mathrm{MHz}$

$1 \mathrm{mM}$ in $\mathrm{D}_{2} \mathrm{O}, 298 \mathrm{~K}$
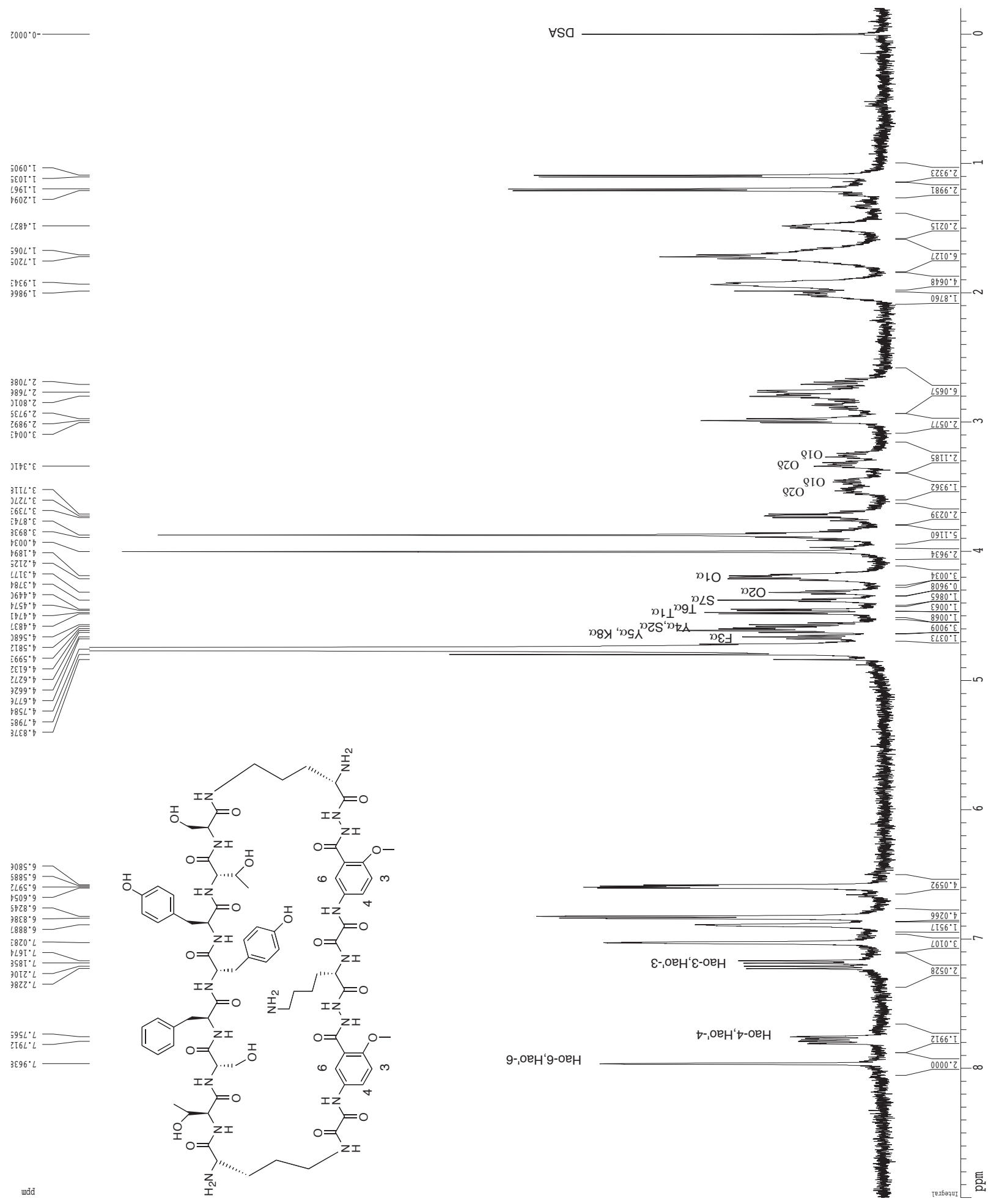

${ }_{356 L}^{39 L L} \cdot L=$

उE96.

wdd 
TOCSY spectrum of the monomer of peptide $\mathbf{3 d}$ $500 \mathrm{MHz}$ (150-ms mixing time)

$1.0 \mathrm{mM}$ in $\mathrm{D}_{2} \mathrm{O}, 298 \mathrm{~K}$

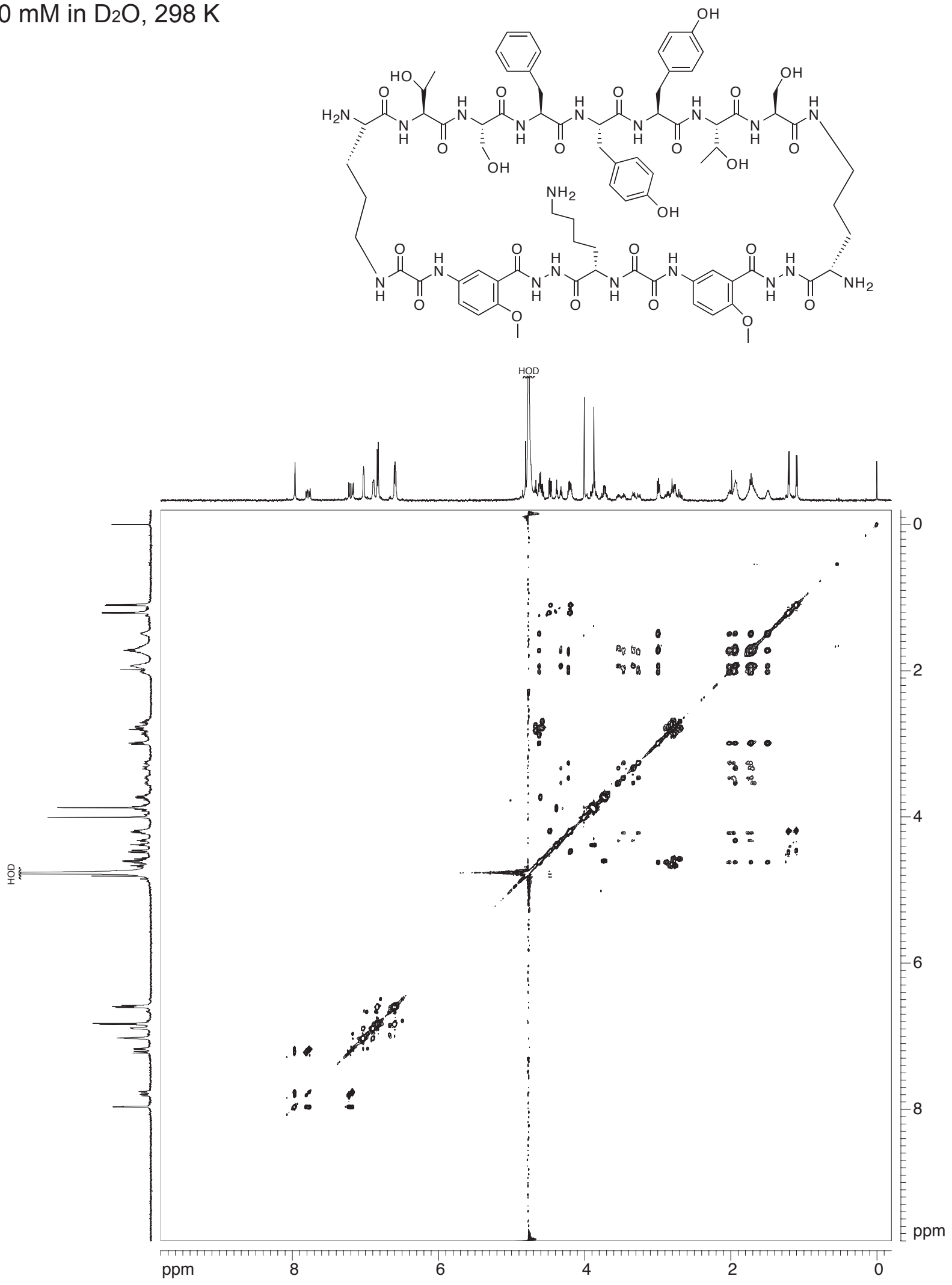


ROESY spectrum of the monomer of peptide $\mathbf{3 d}$ $500 \mathrm{MHz}$ (300-ms mixing time)

\section{$1.0 \mathrm{mM}$ in $\mathrm{D}_{2} \mathrm{O}, 298 \mathrm{~K}$}

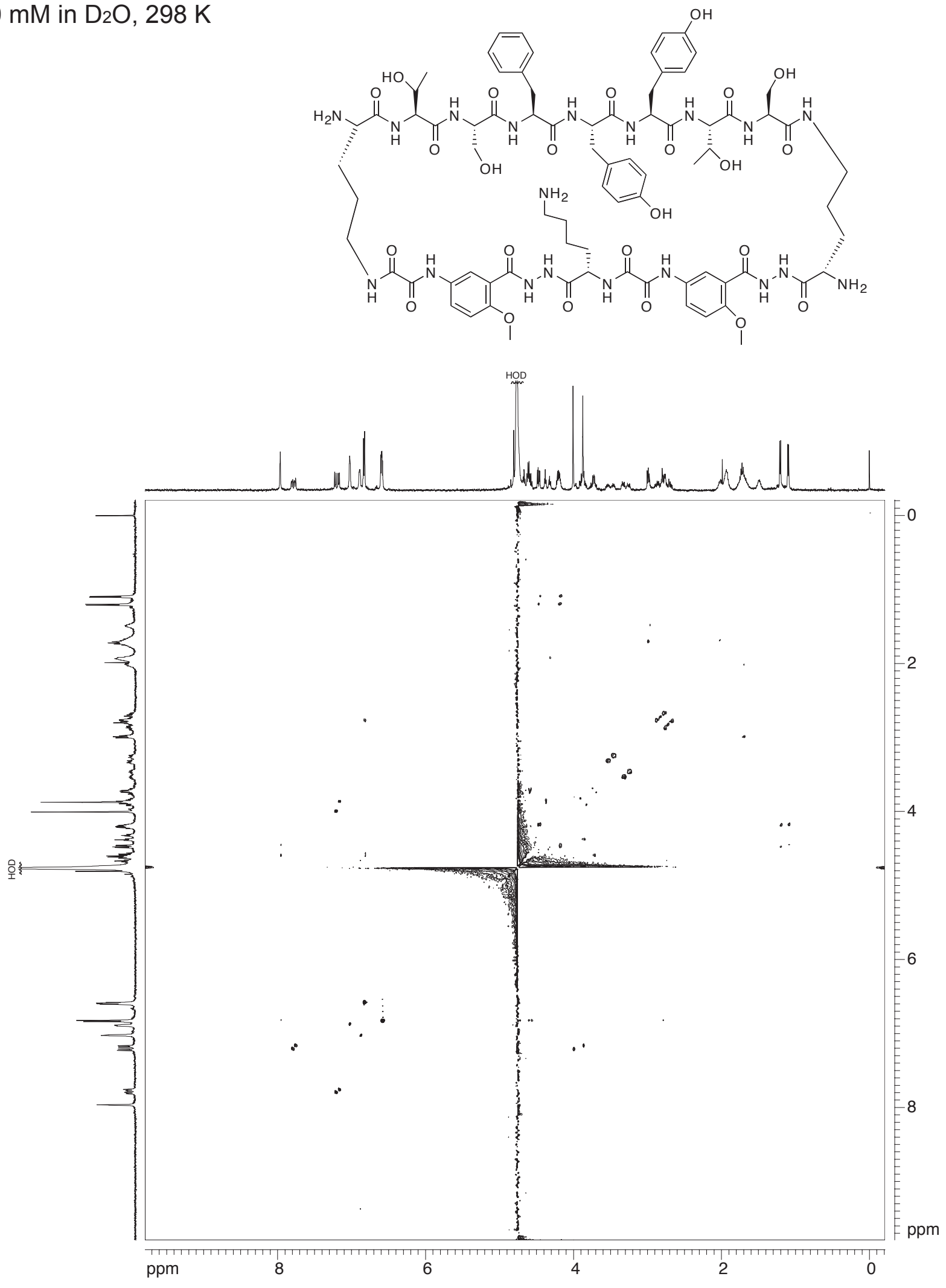


Overlay of $1 \mathrm{D}{ }^{1} \mathrm{H}$ NMR spectra of dilute and concentrated solutions of peptide $\mathbf{3 d}$ $500 \mathrm{MHz}$

$\mathrm{D}_{2} \mathrm{O}, 298 \mathrm{~K}$
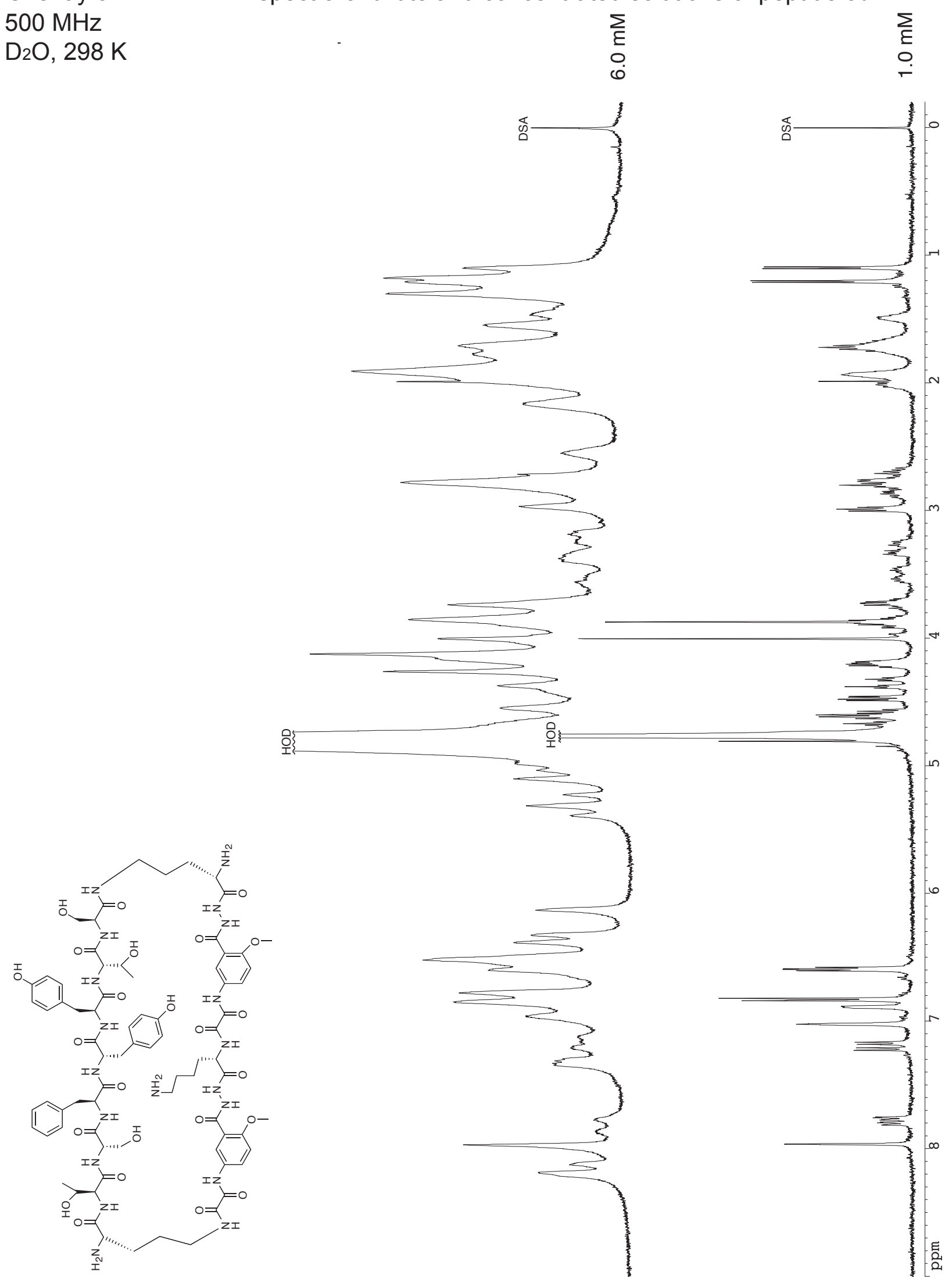
1D ${ }^{1} \mathrm{H}$ NMR spectrum of the monomer of peptide $3 e$

$500 \mathrm{MHz}$

$1 \mathrm{mM}$ in $\mathrm{D}_{2} \mathrm{O}, 298 \mathrm{~K}$
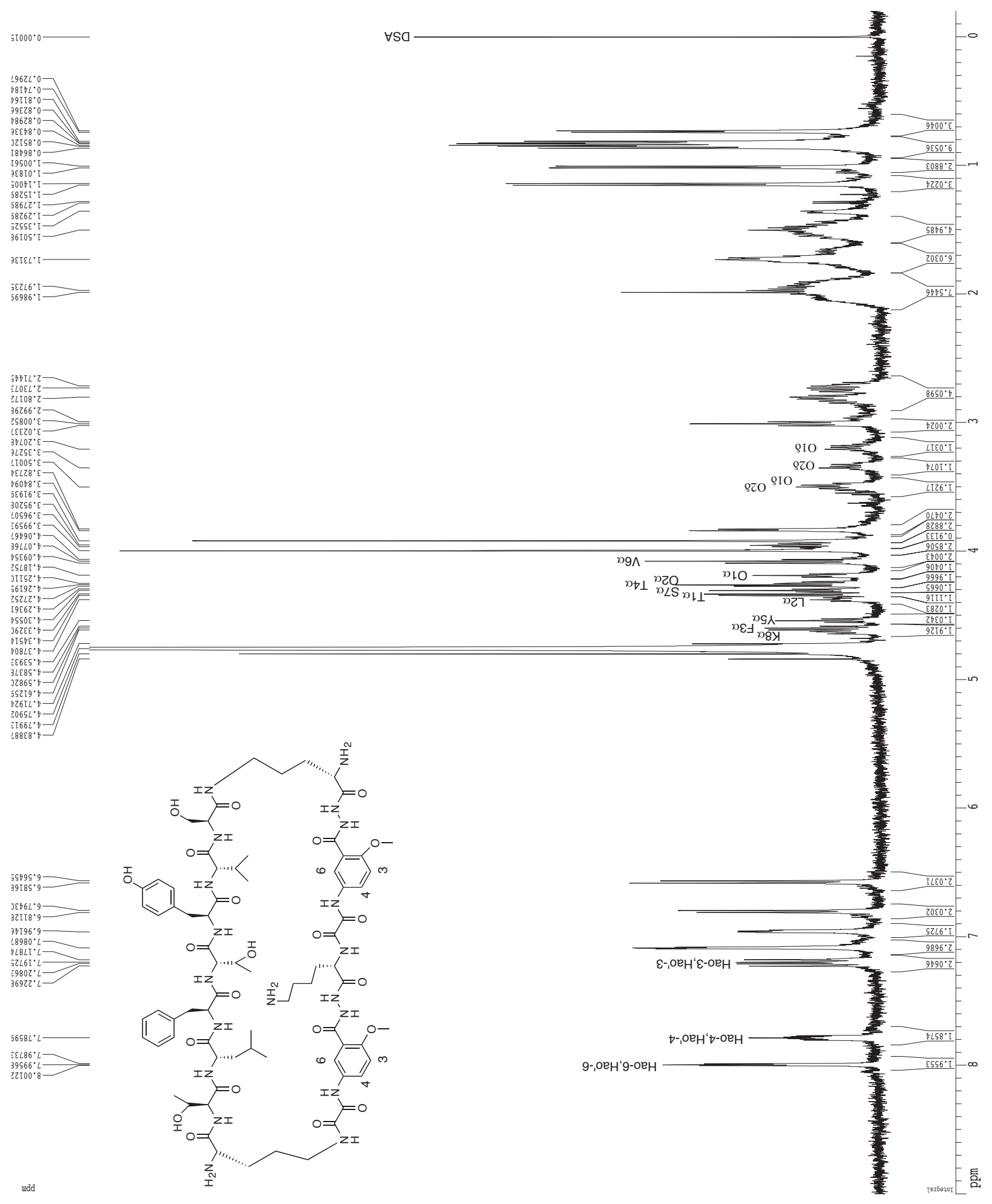
TOCSY spectrum of the monomer of peptide $\mathbf{3 e}$ $500 \mathrm{MHz}$ (150-ms mixing time)

$1.0 \mathrm{mM}$ in $\mathrm{D}_{2} \mathrm{O}, 298 \mathrm{~K}$
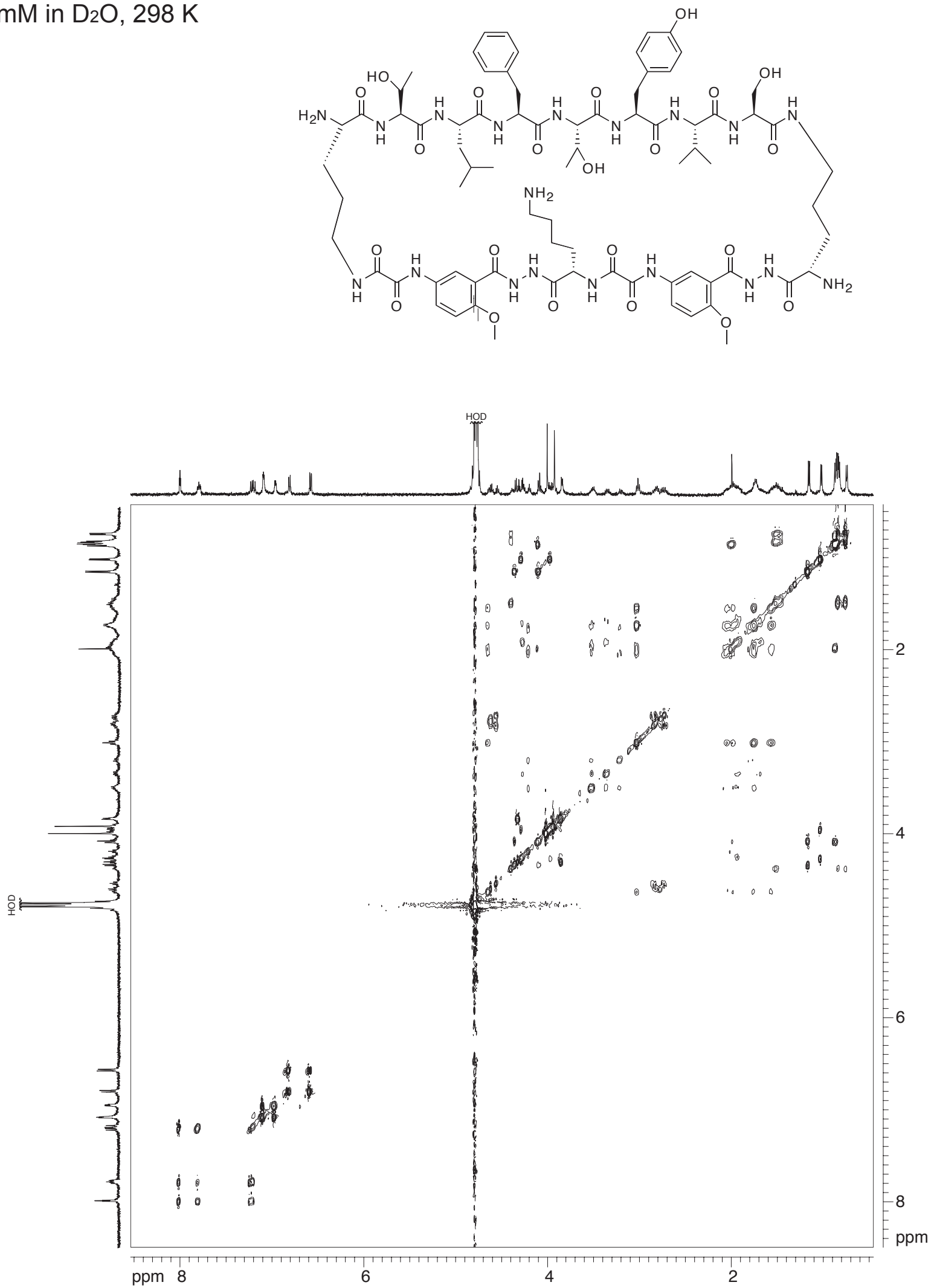
ROESY spectrum of the monomer of peptide $3 e$ $500 \mathrm{MHz}$ (300-ms mixing time)

$1.0 \mathrm{mM}$ in $\mathrm{D}_{2} \mathrm{O}, 298 \mathrm{~K}$

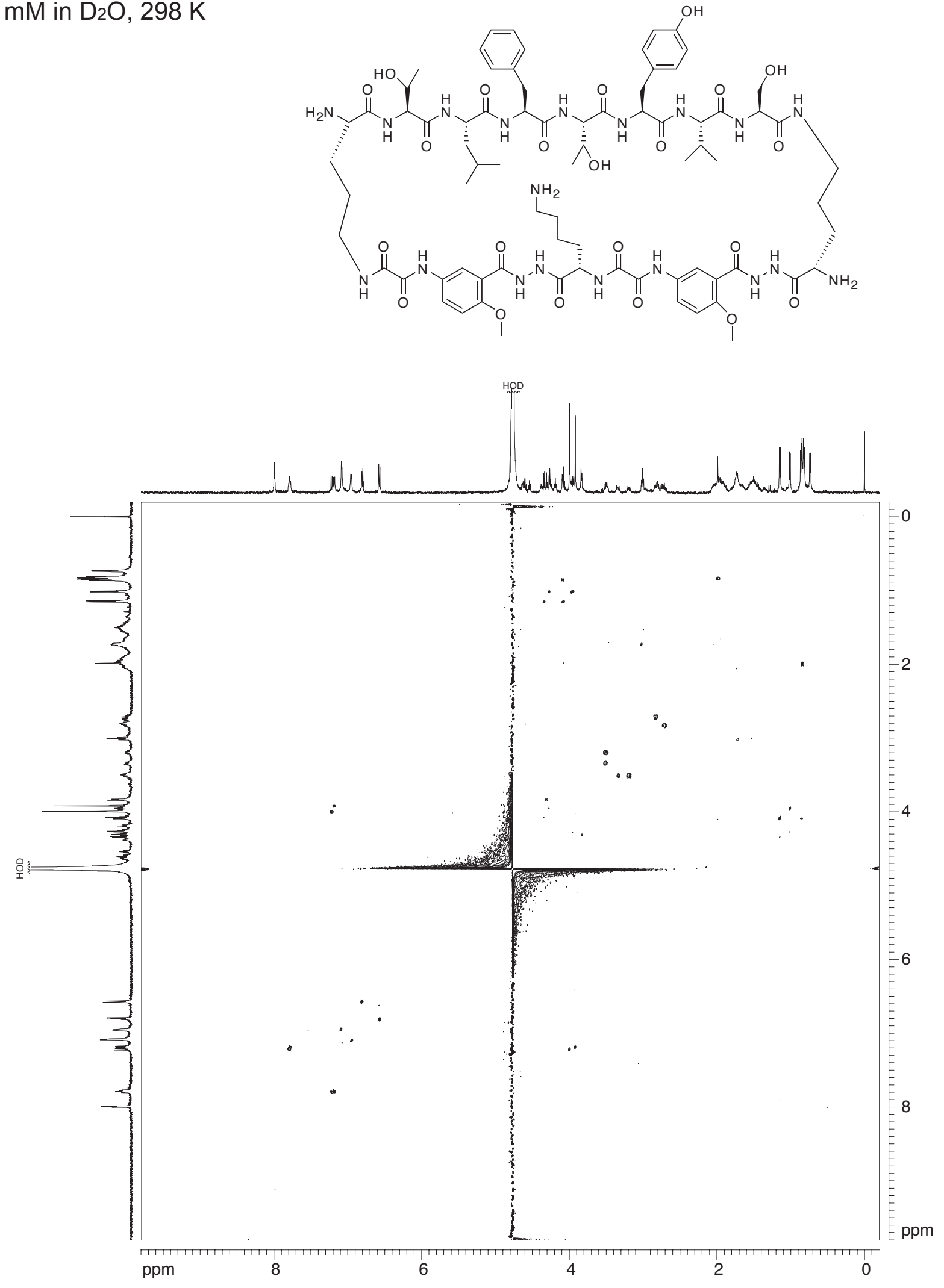


Overlay of $1 \mathrm{D}{ }^{1} \mathrm{H}$ NMR spectra of dilute and concentrated solutions of peptide $3 e$ $500 \mathrm{MHz}$

$\mathrm{D}_{2} \mathrm{O}, 298 \mathrm{~K}$
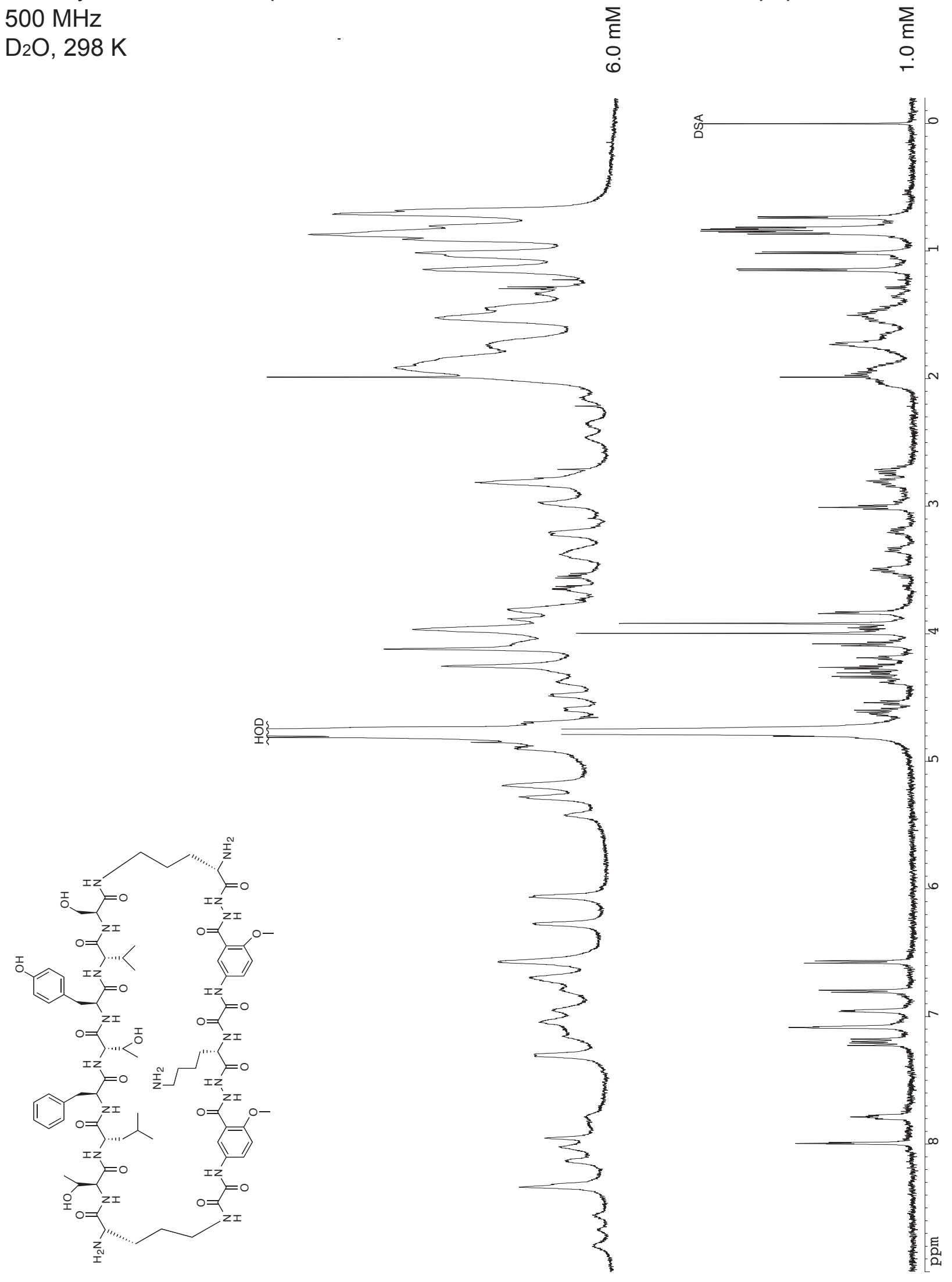
1D ${ }^{1} \mathrm{H}$ NMR spectrum of the monomer of peptide $3 f$

$500 \mathrm{MHz}$

$0.5 \mathrm{mM}$ in $\mathrm{D}_{2} \mathrm{O}, 298 \mathrm{~K}$
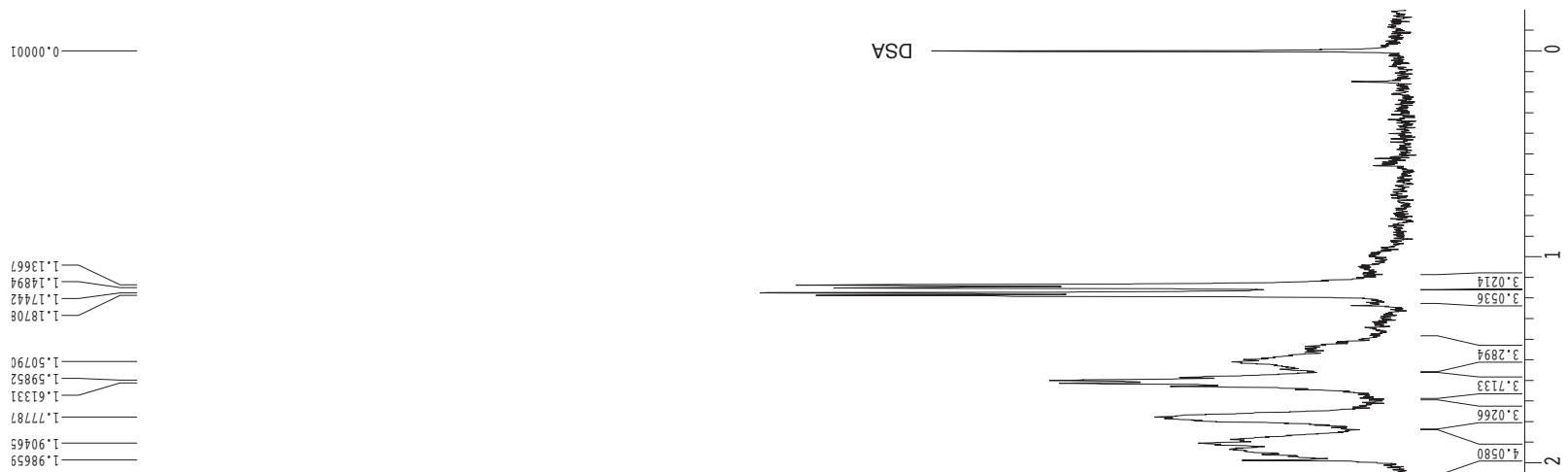

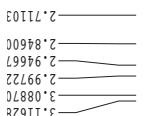

$\operatorname{lit}^{3} \cos \tau^{\circ} \varepsilon$

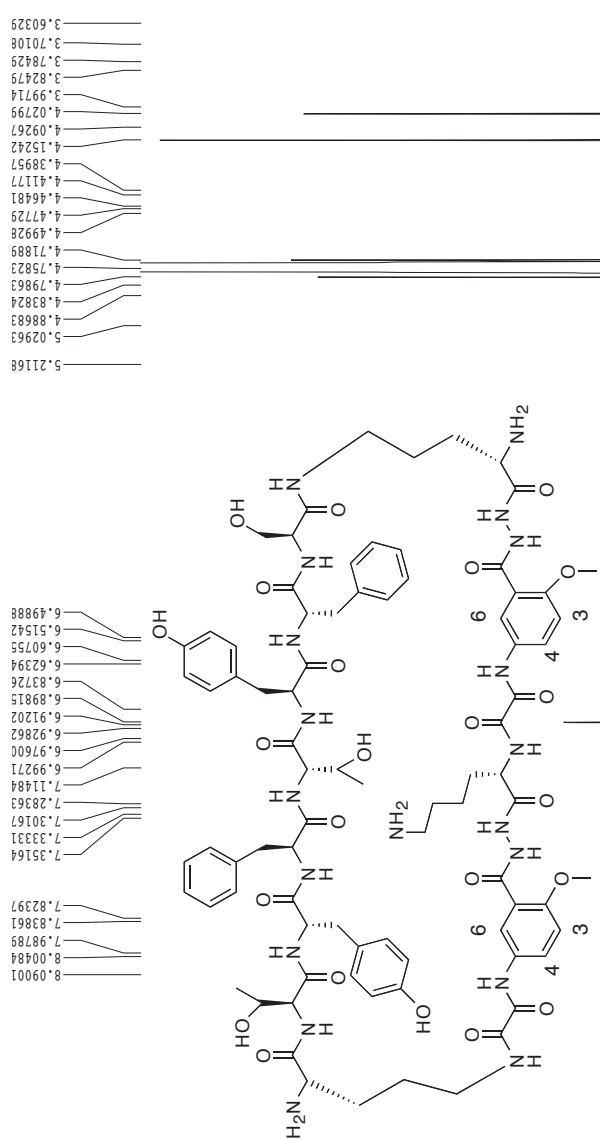

wdd

머ำ

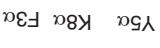
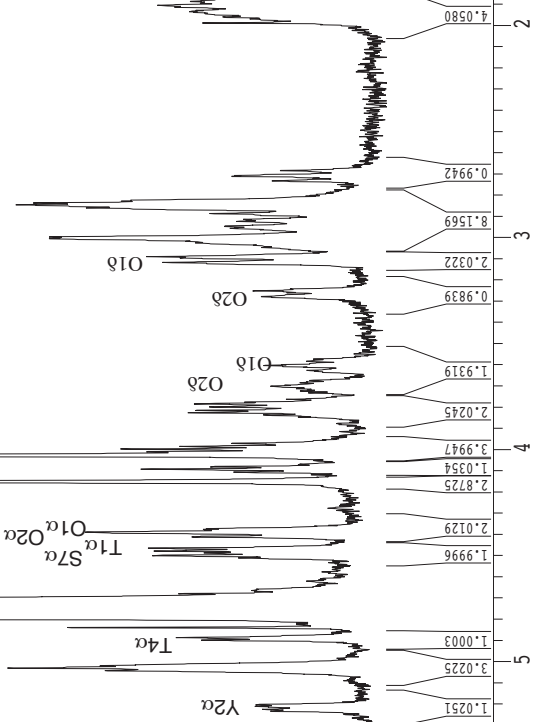
TOCSY spectrum of the monomer of peptide $\mathbf{3 f}$ $500 \mathrm{MHz}$ (150-ms mixing time)

$0.7 \mathrm{mM}$ in $\mathrm{D}_{2} \mathrm{O}, 298 \mathrm{~K}$

Traces of the oligomer is also visible
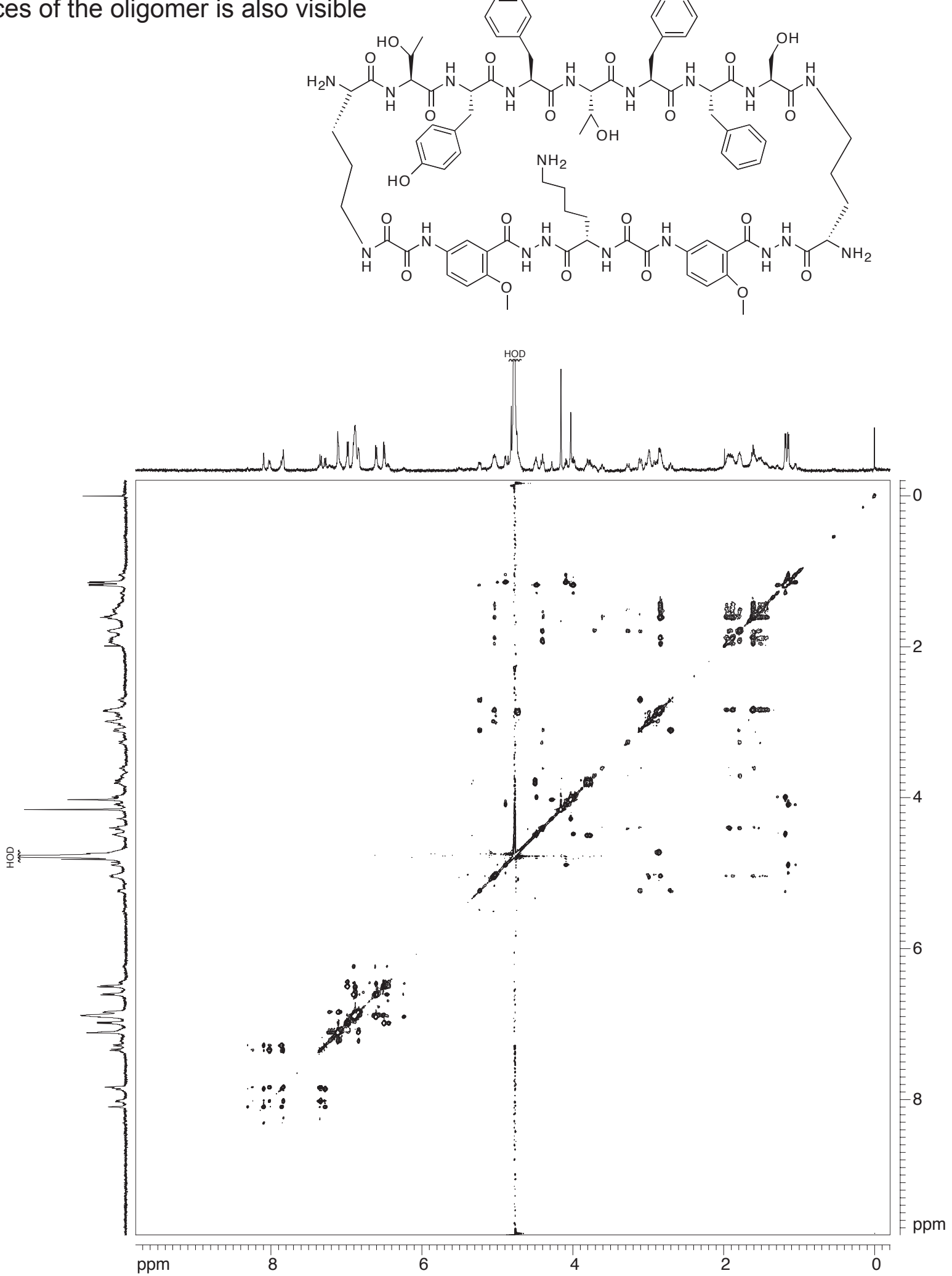
ROESY spectrum of the monomer of peptide $\mathbf{3 f}$ $500 \mathrm{MHz}$ (300-ms mixing time)

$0.7 \mathrm{mM}$ in $\mathrm{D}_{2} \mathrm{O}, 298 \mathrm{~K}$

Traces of the oligomer is also visible

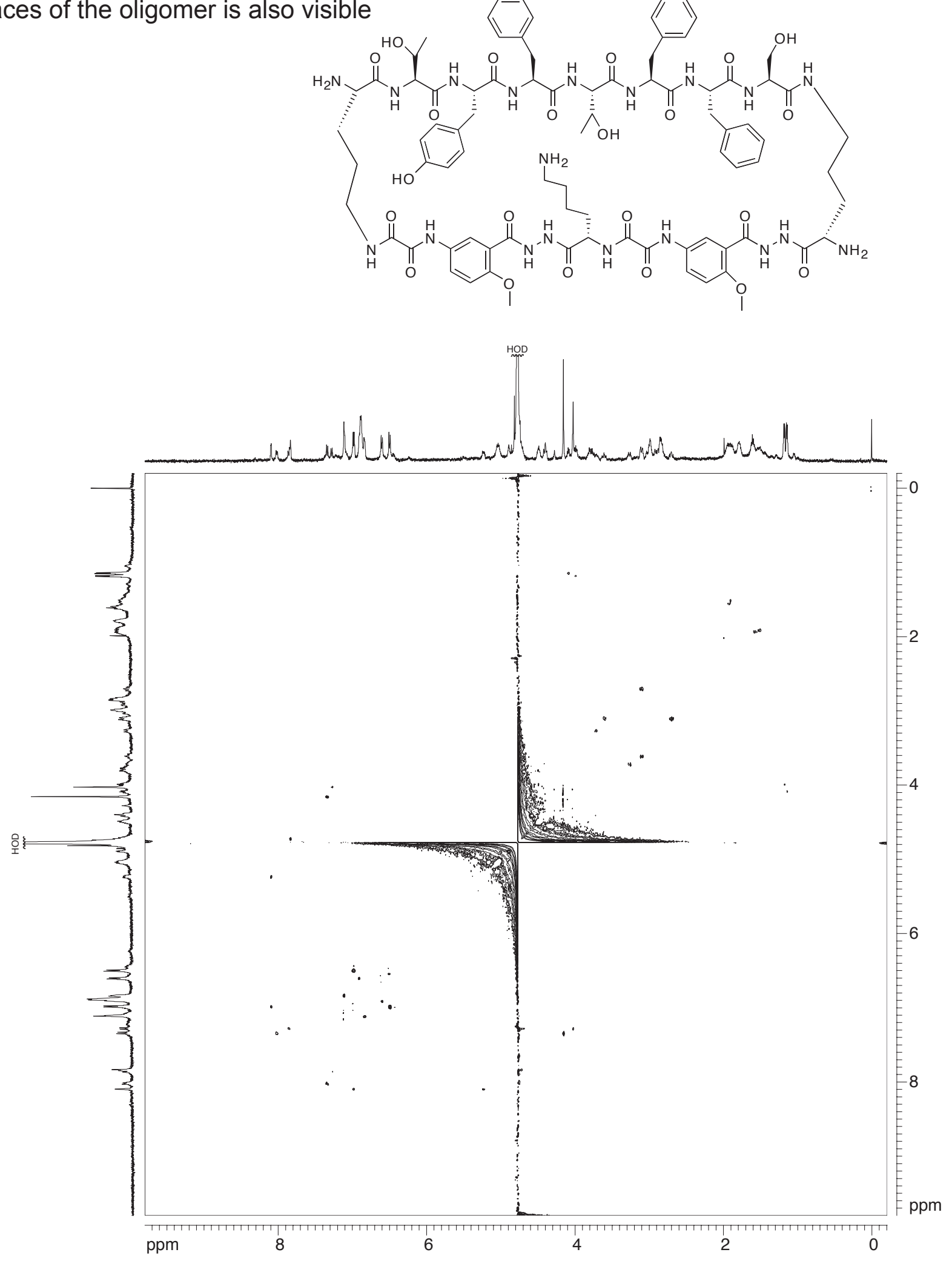


Overlay of $1 \mathrm{D}{ }^{1} \mathrm{H}$ NMR spectra of dilute and concentrated solutions of peptide $\mathbf{3 f}$ $500 \mathrm{MHz}$

$\mathrm{D}_{2} \mathrm{O}, 298 \mathrm{~K}$
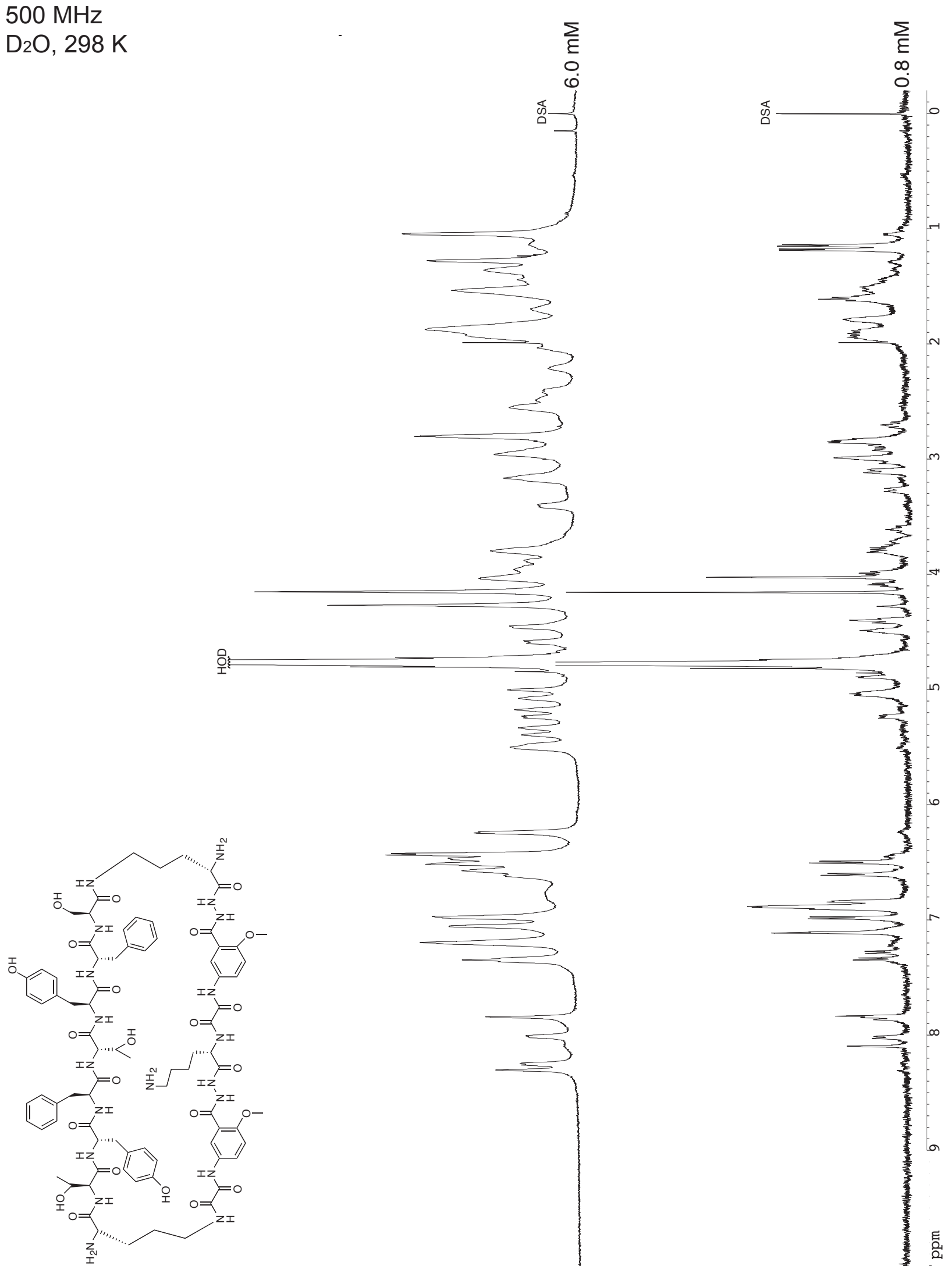
1D ${ }^{1} \mathrm{H}$ NMR spectrum of the monomer of peptide $3 \mathrm{~g}$

$500 \mathrm{MHz}$

$0.5 \mathrm{mM}$ in $\mathrm{D}_{2} \mathrm{O}, 298 \mathrm{~K}$
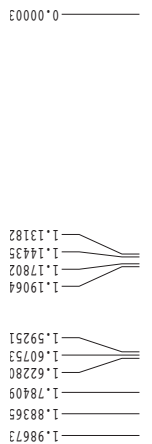

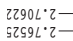

$9208 L^{\circ} \mathrm{Z}=$

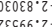

$9 \angle 60 \top^{\circ} \varepsilon$

$\varepsilon 68 L \tau^{\circ} \varepsilon$
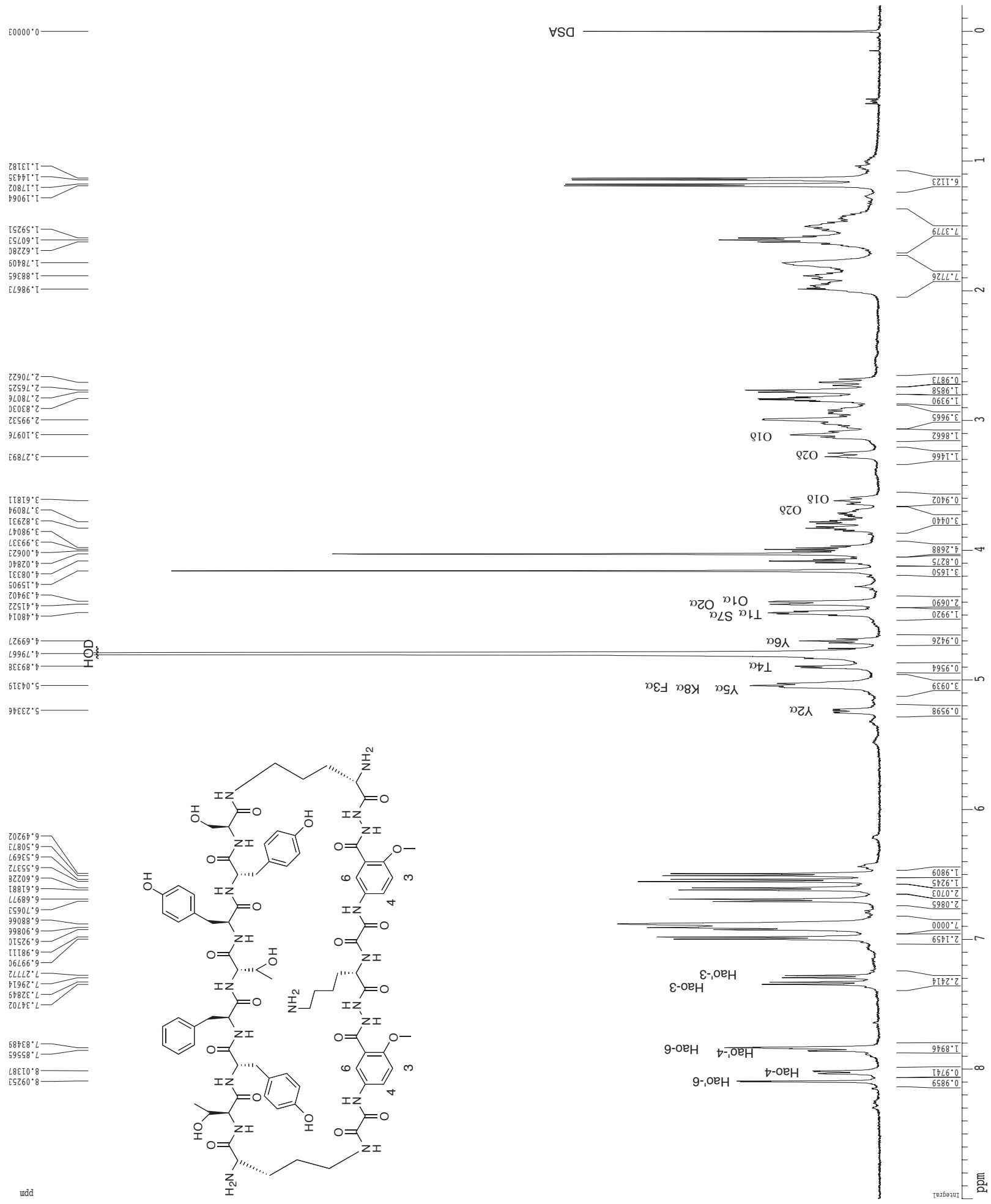
TOCSY spectrum of the monomer of peptide $\mathbf{3 g}$ $500 \mathrm{MHz}$ Cryoprobe (150-ms mixing time)

$0.5 \mathrm{mM}$ in $\mathrm{D}_{2} \mathrm{O}, 298 \mathrm{~K}$

Traces of the oligomer is also visible

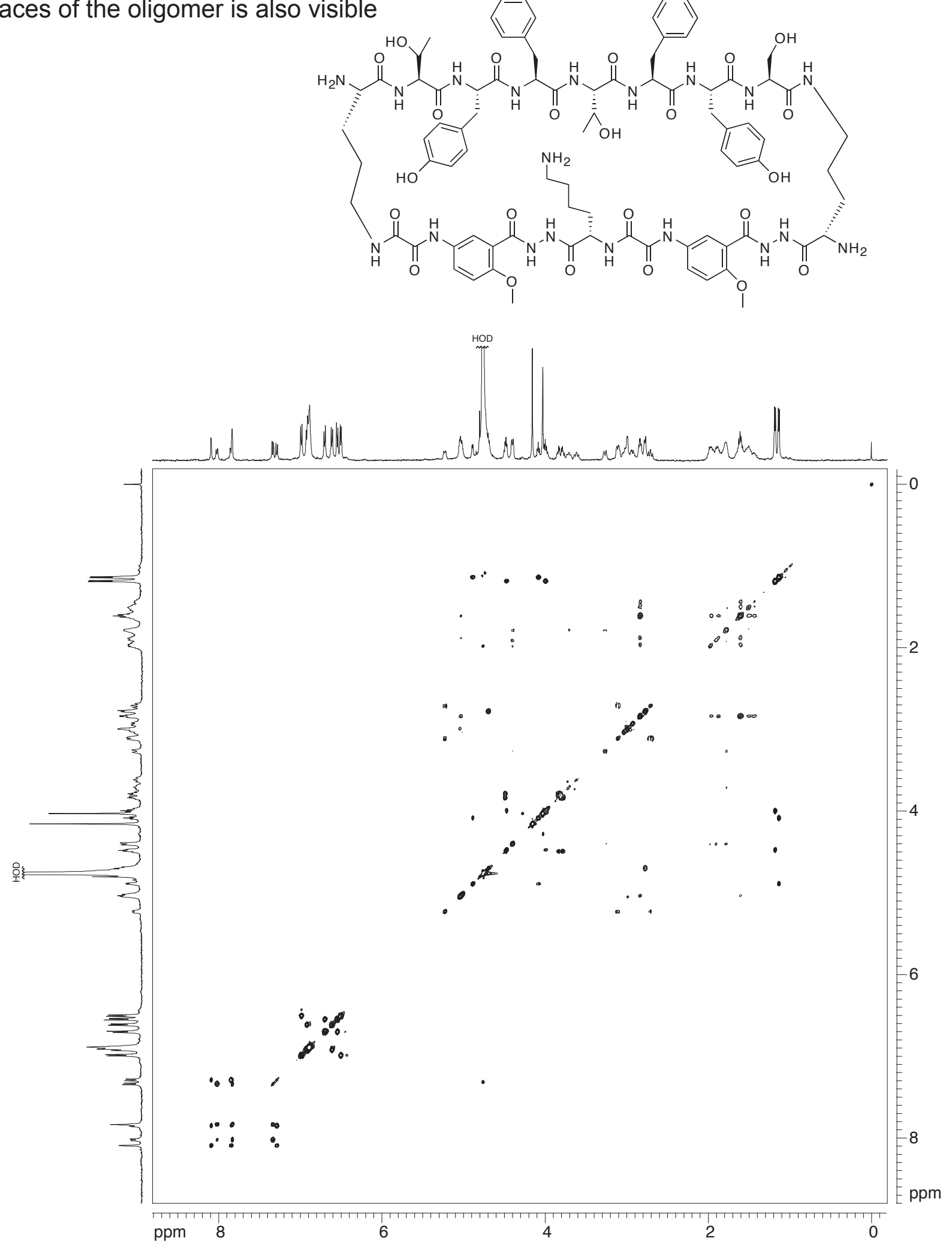


ROESY spectrum of the monomer of peptide $3 \mathrm{~g}$ $500 \mathrm{MHz}$ Cryo (300-ms mixing time) $0.5 \mathrm{mM}$ in $\mathrm{D}_{2} \mathrm{O}, 298 \mathrm{~K}$

Traces of the oligomer is also visible
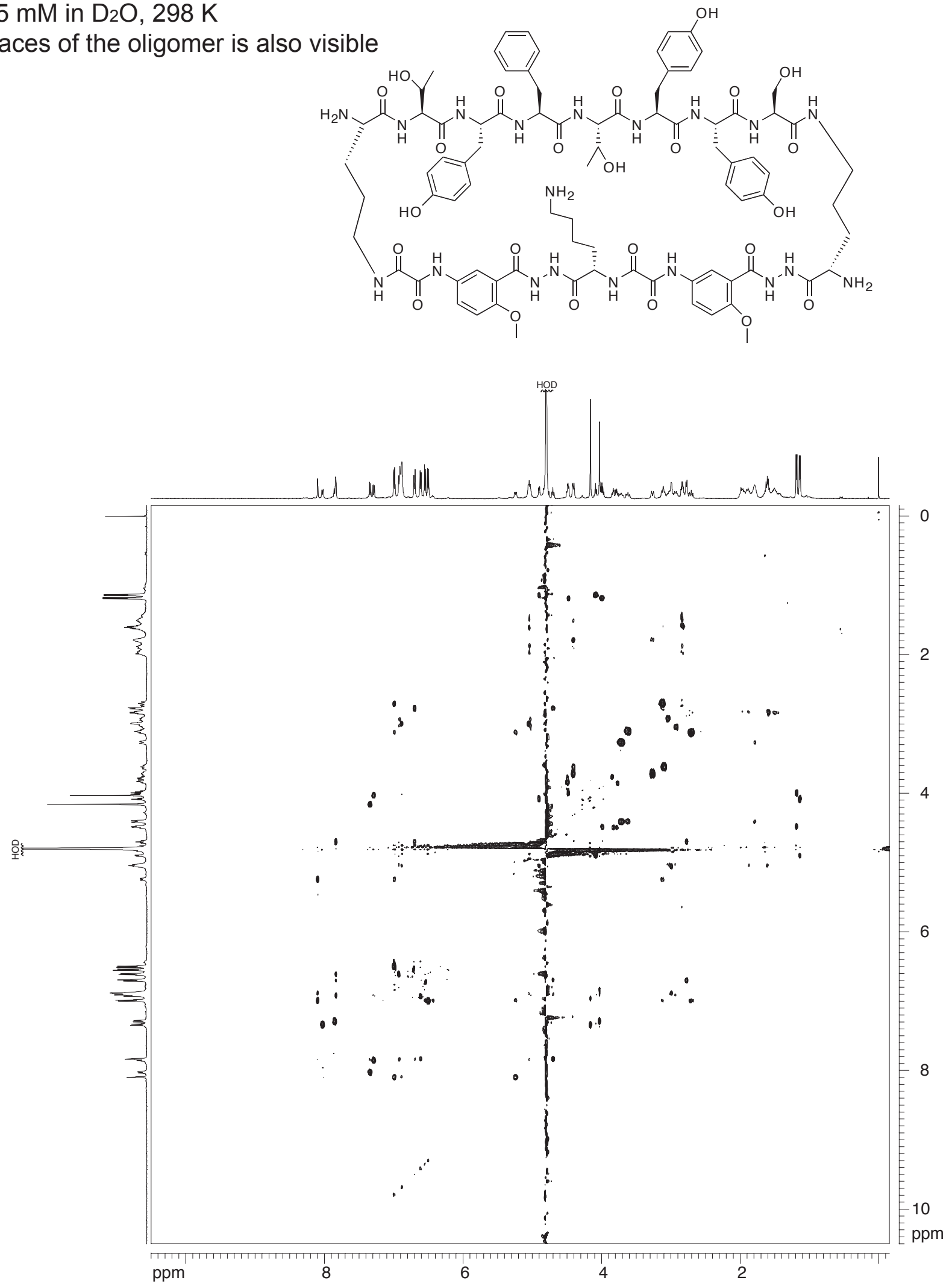
Overlay of $1 \mathrm{D}{ }^{1} \mathrm{H}$ NMR spectra of dilute and concentrated solutions of peptide $\mathbf{3 g}$ $500 \mathrm{MHz}$ cryoprobe

$\mathrm{D}_{2} \mathrm{O}, 298 \mathrm{~K}$

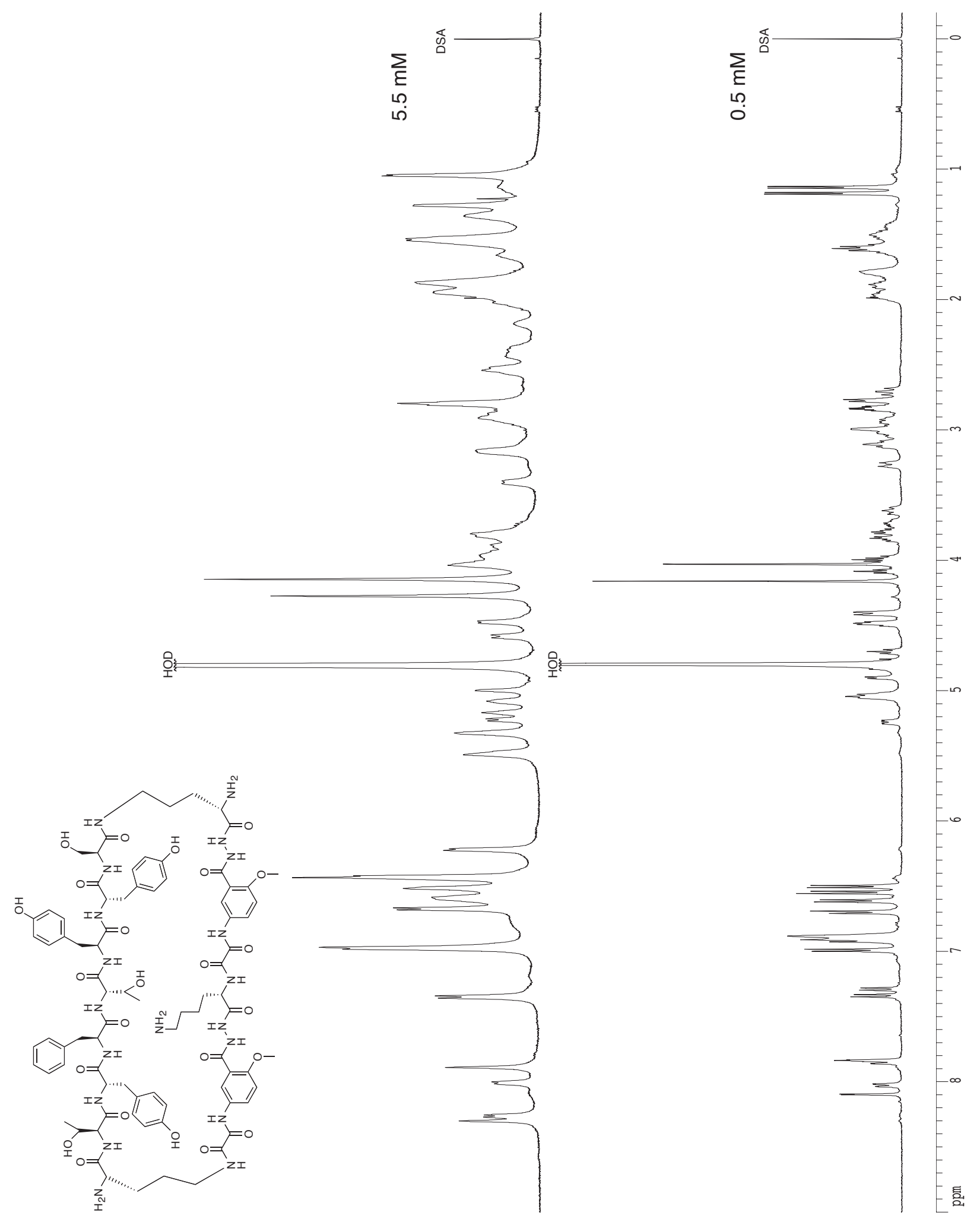


1D ${ }^{1} \mathrm{H}$ NMR spectrum of the monomer of peptide $3 \mathrm{~h}$

$500 \mathrm{MHz}$

$1.0 \mathrm{mM}$ in $\mathrm{D}_{2} \mathrm{O}, 298 \mathrm{~K}$
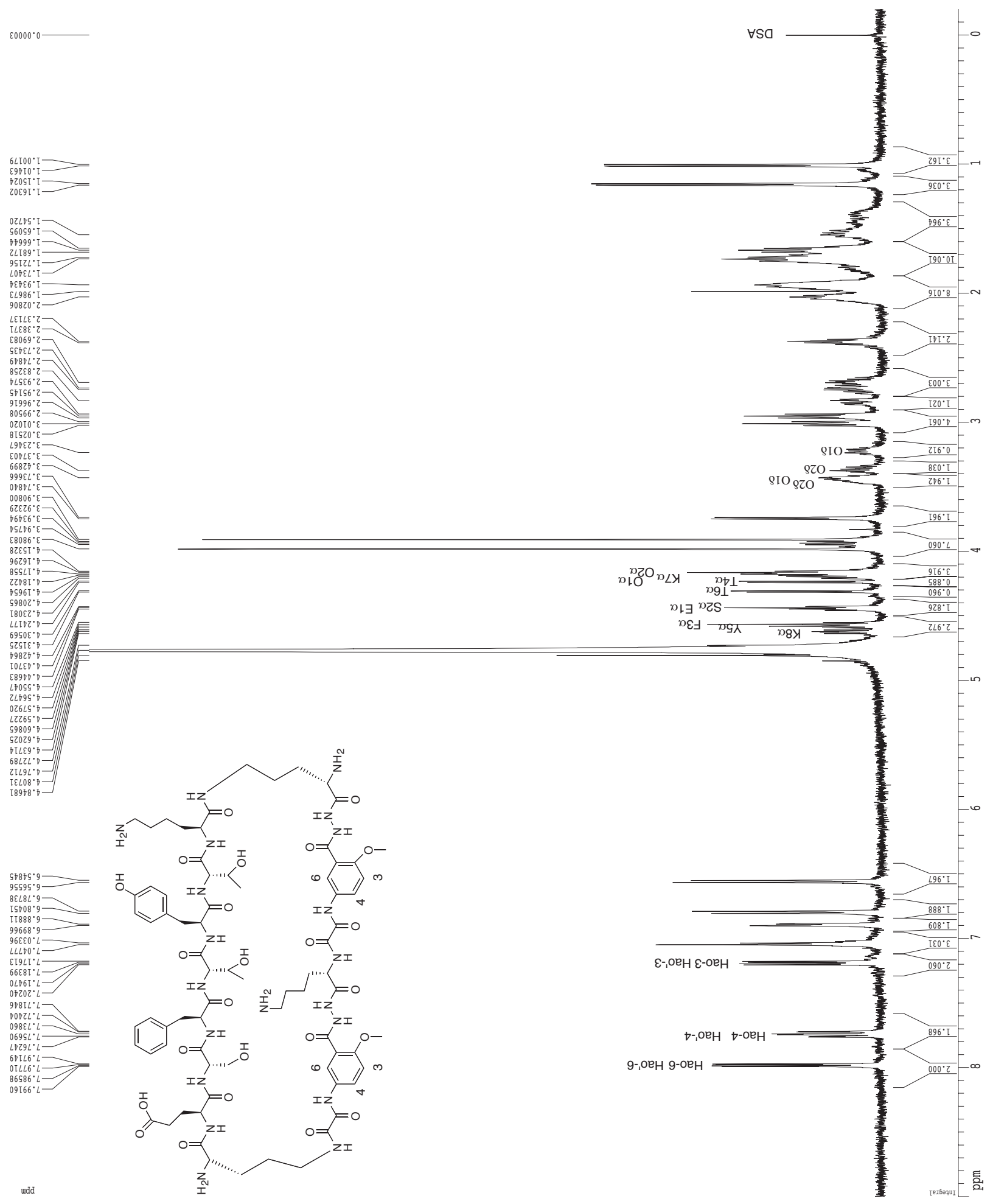
TOCSY spectrum of the monomer of peptide $\mathbf{3 h}$ $500 \mathrm{MHz}$ (150-ms mixing time) $1.0 \mathrm{mM}$ in $\mathrm{D}_{2} \mathrm{O}, 298 \mathrm{~K}$

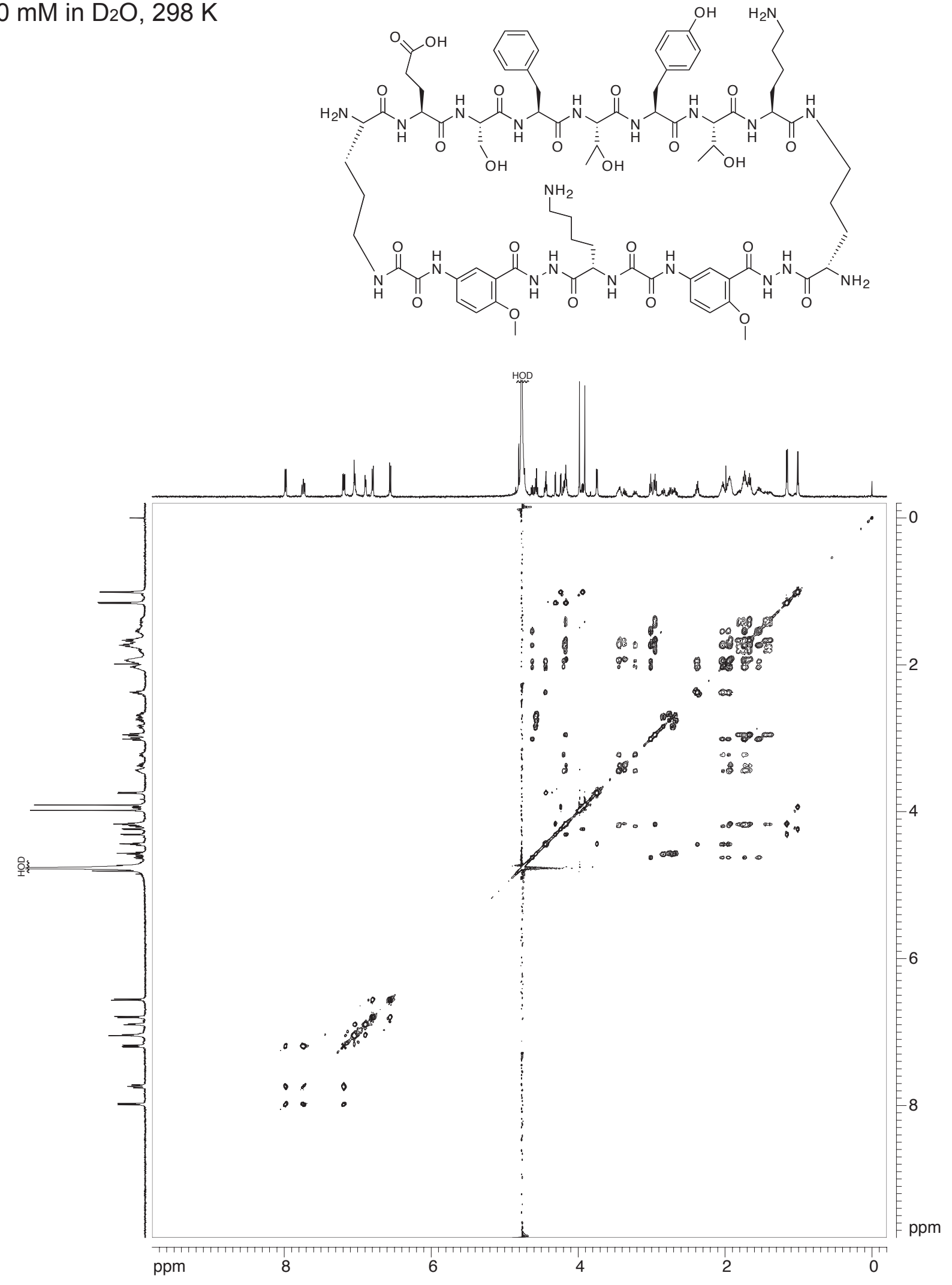


ROESY spectrum of the monomer of peptide $3 \mathrm{~h}$ $500 \mathrm{MHz}$ (300-ms mixing time)

\section{$1.0 \mathrm{mM}$ in $\mathrm{D}_{2} \mathrm{O}, 298 \mathrm{~K}$}

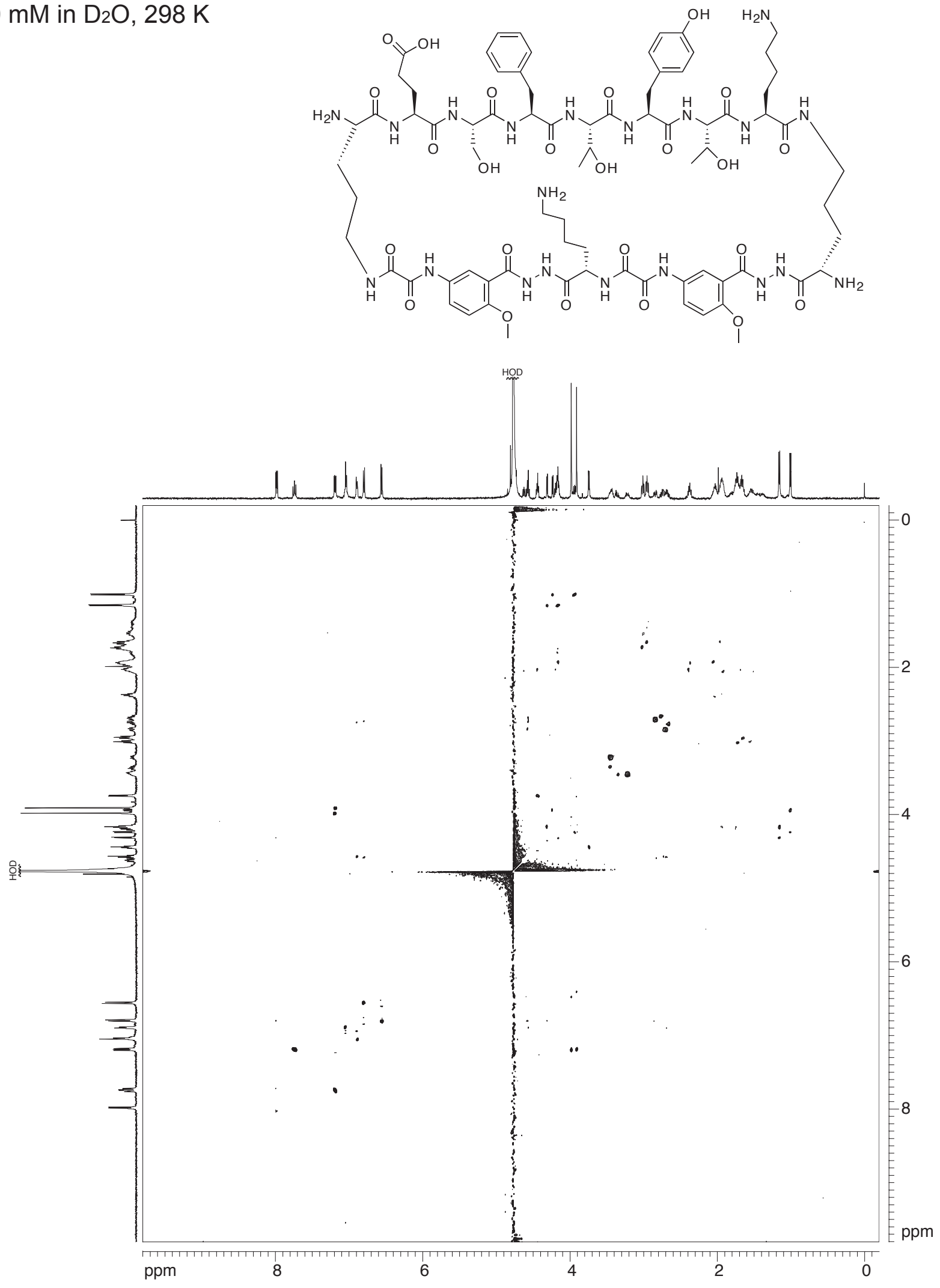


Overlay of $1 \mathrm{D}{ }^{1} \mathrm{H}$ NMR spectra of dilute and concentrated solutions of peptide $3 \mathrm{~h}$ $500 \mathrm{MHz}$

$\mathrm{D}_{2} \mathrm{O}$

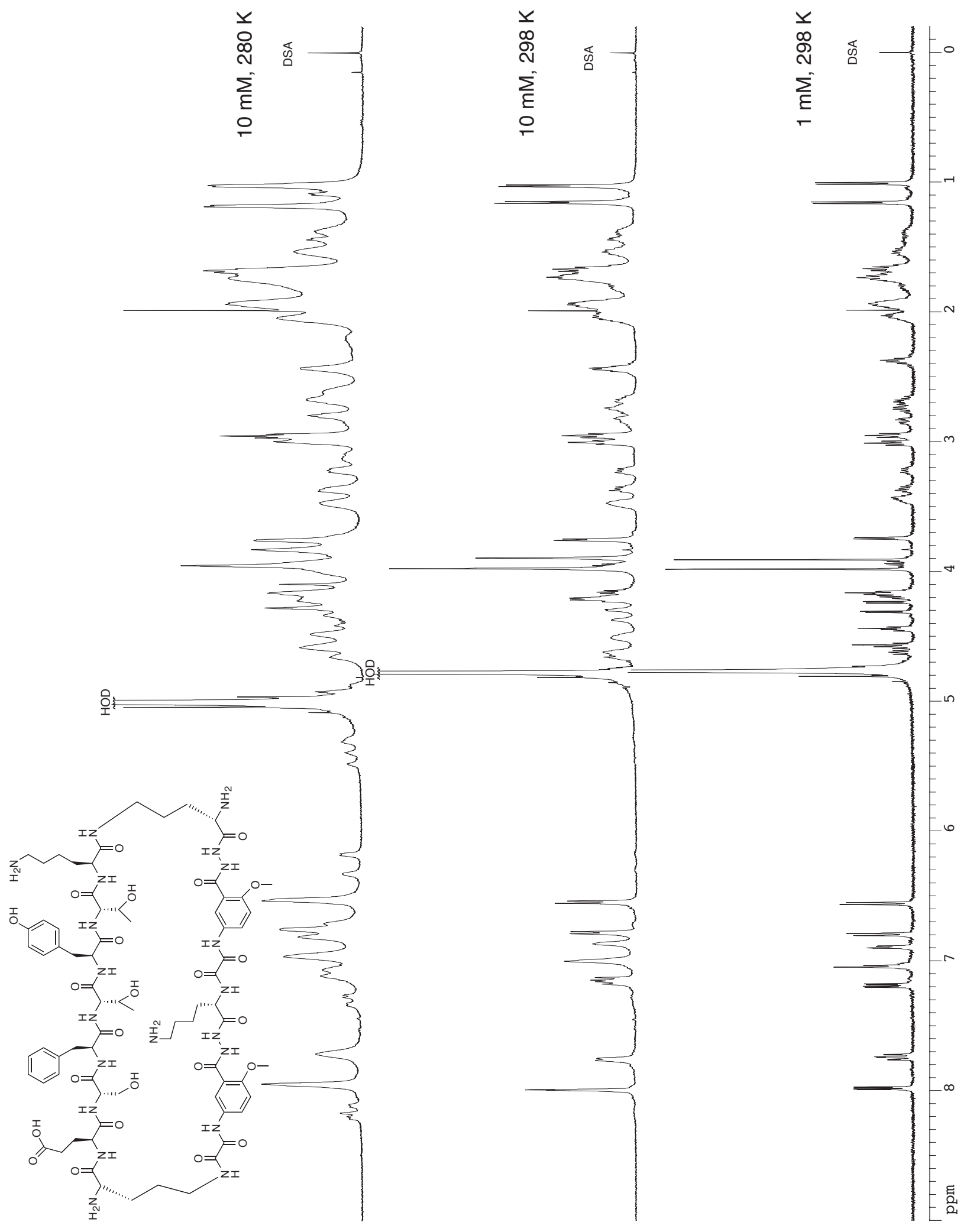


1D ${ }^{1} \mathrm{H}$ NMR spectrum of peptide $\mathbf{3 i}$

$500 \mathrm{MHz}$

$1.0 \mathrm{mM}$ in $\mathrm{D}_{2} \mathrm{O}, 298 \mathrm{~K}$

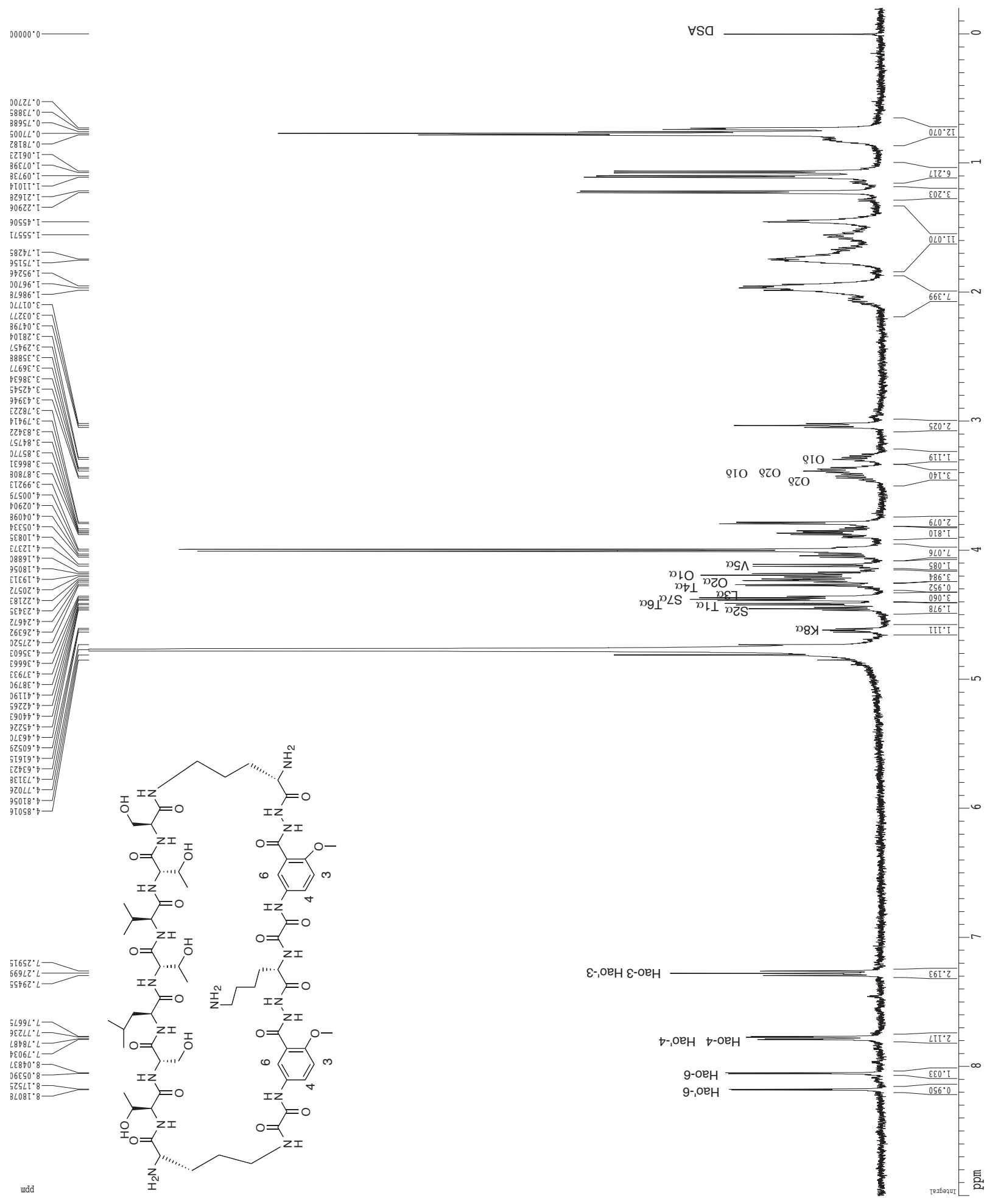


ROESY spectrum of peptide $\mathbf{3 i}$

$500 \mathrm{MHz}$ (150-ms mixing time)

$1.0 \mathrm{mM}$ in $\mathrm{D}_{2} \mathrm{O}, 298 \mathrm{~K}$

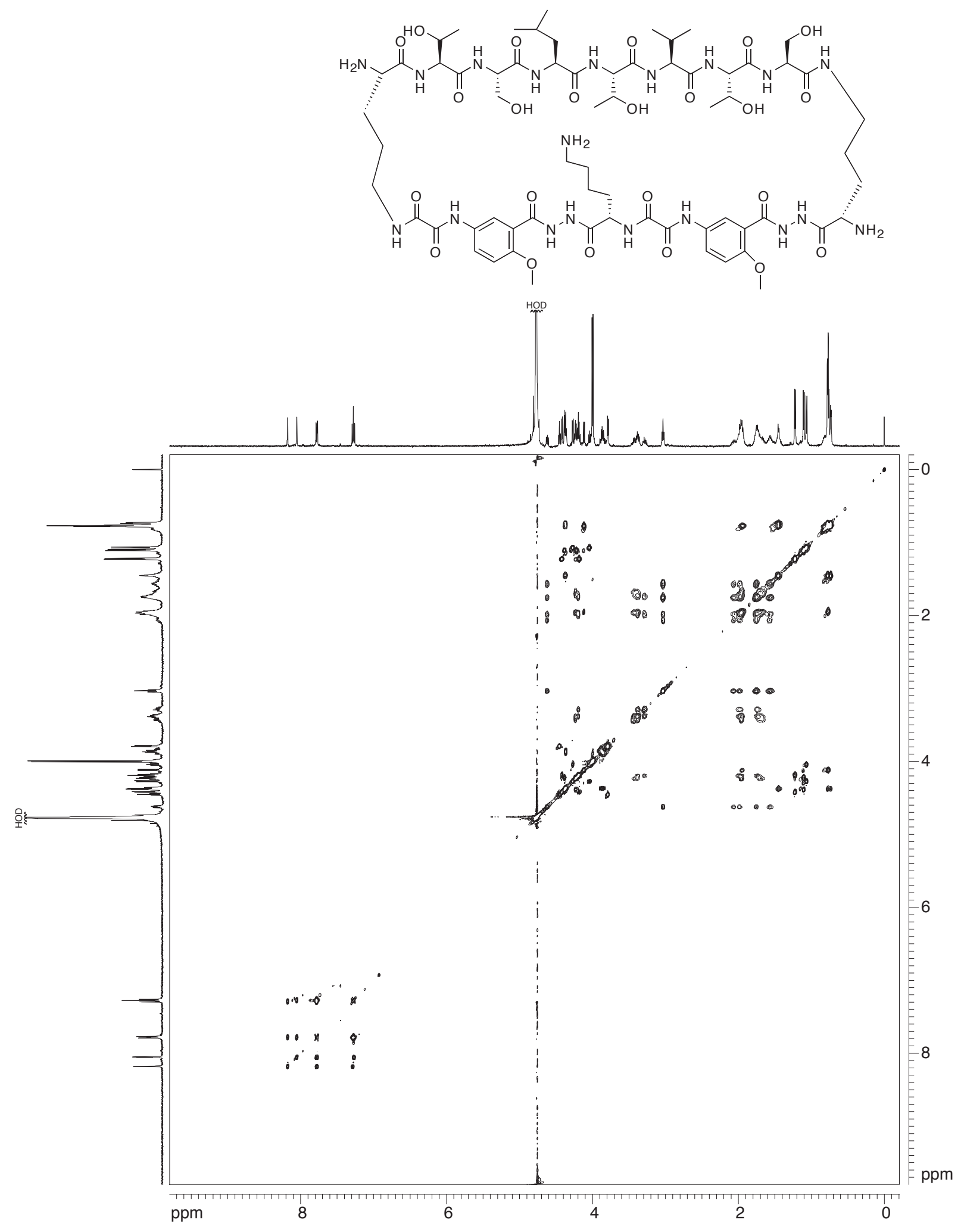


ROESY spectrum of peptide $\mathbf{3 i}$

$500 \mathrm{MHz}$ (300-ms mixing time)

$1.0 \mathrm{mM}$ in $\mathrm{D}_{2} \mathrm{O}, 298 \mathrm{~K}$

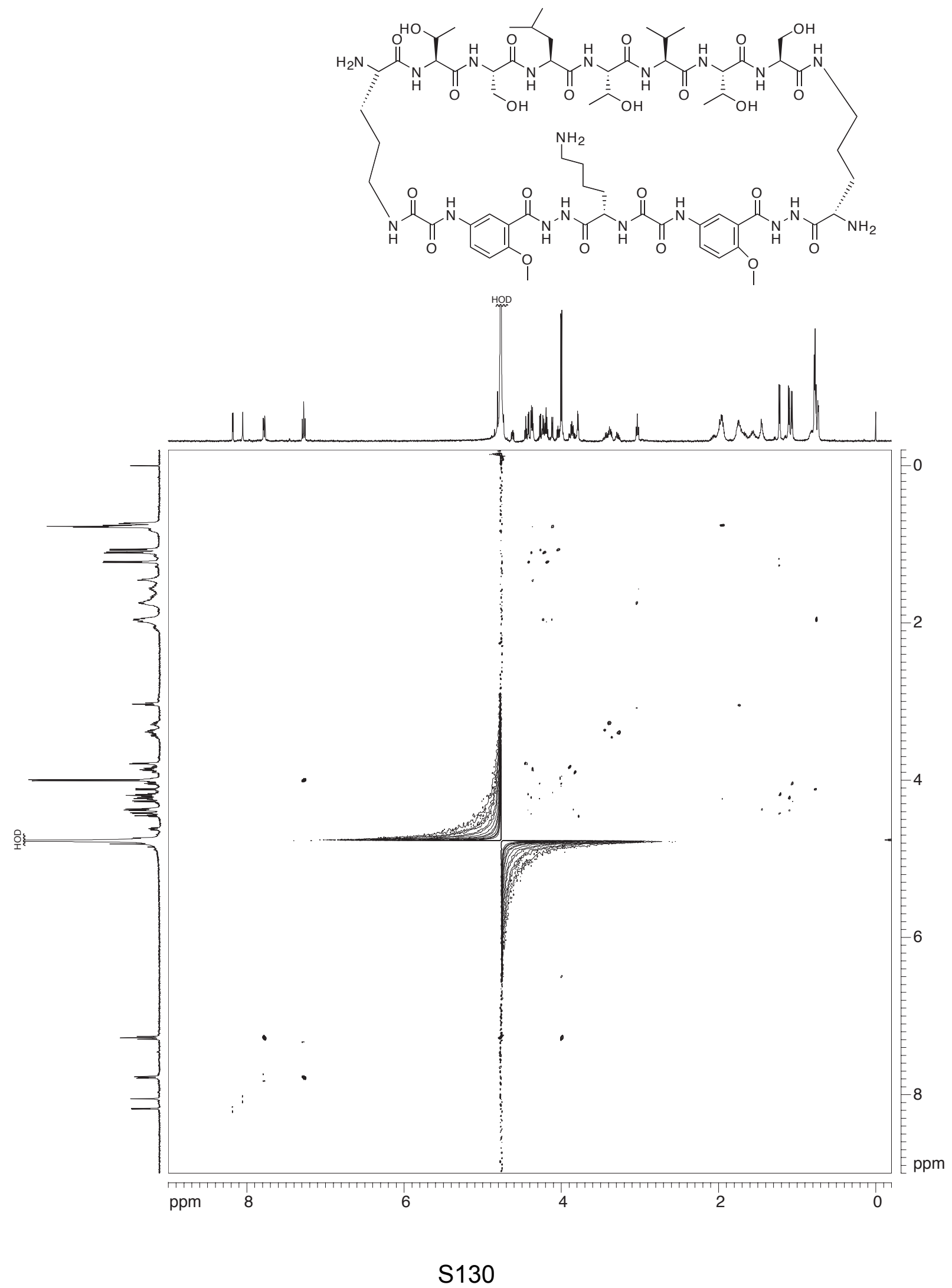


Overlay of $1 \mathrm{D}{ }^{1} \mathrm{H}$ NMR spectra of dilute and concentrated solutions of peptide $3 \mathbf{i}$ $500 \mathrm{MHz}$

$\mathrm{D}_{2} \mathrm{O}$

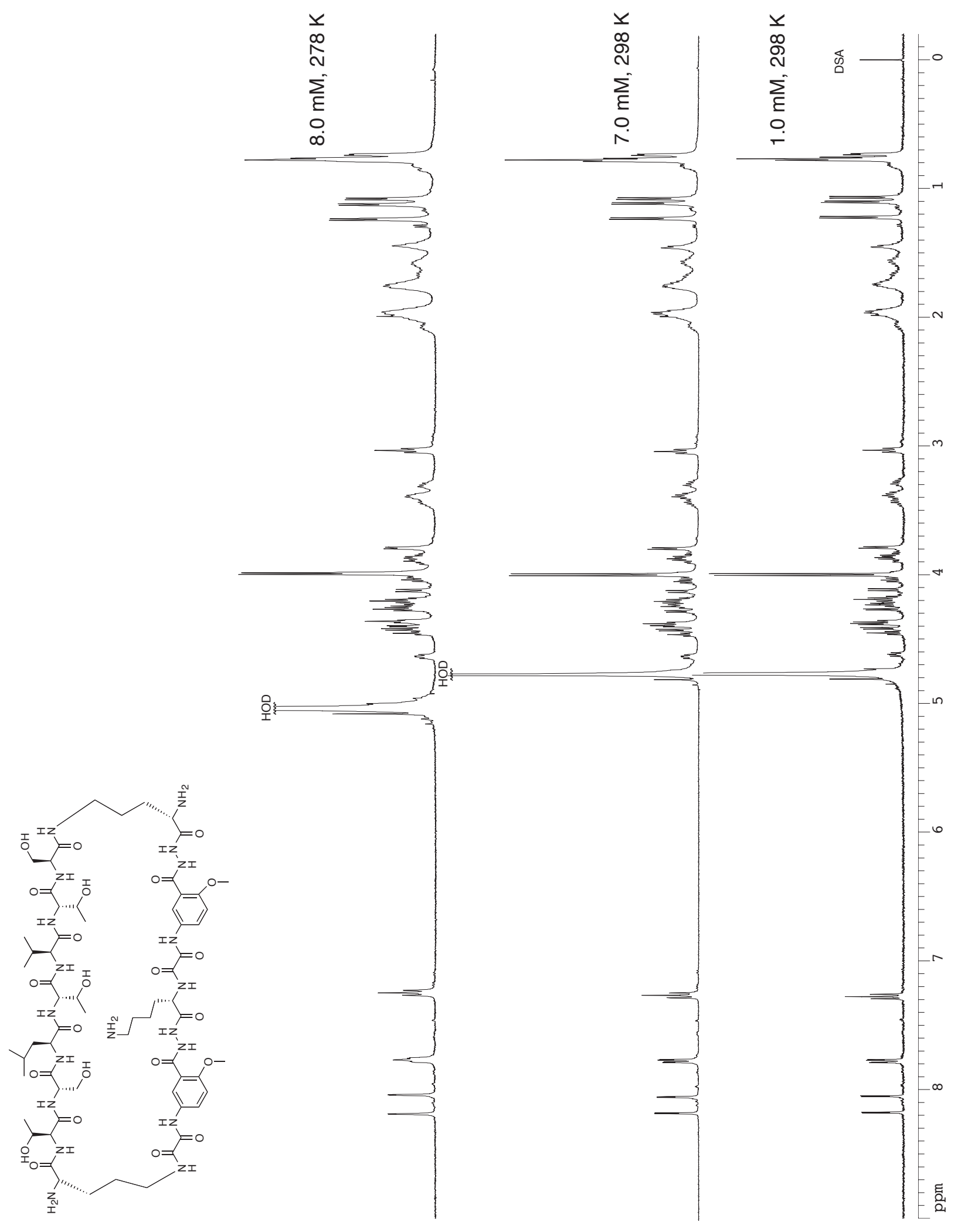


1D ${ }^{1} \mathrm{H}$ NMR spectrum of the monomer of peptide $3 \mathbf{j}$

$500 \mathrm{MHz}$ Cryoprobe

$0.4 \mathrm{mM}$ in $\mathrm{D}_{2} \mathrm{O}, 298 \mathrm{~K}$

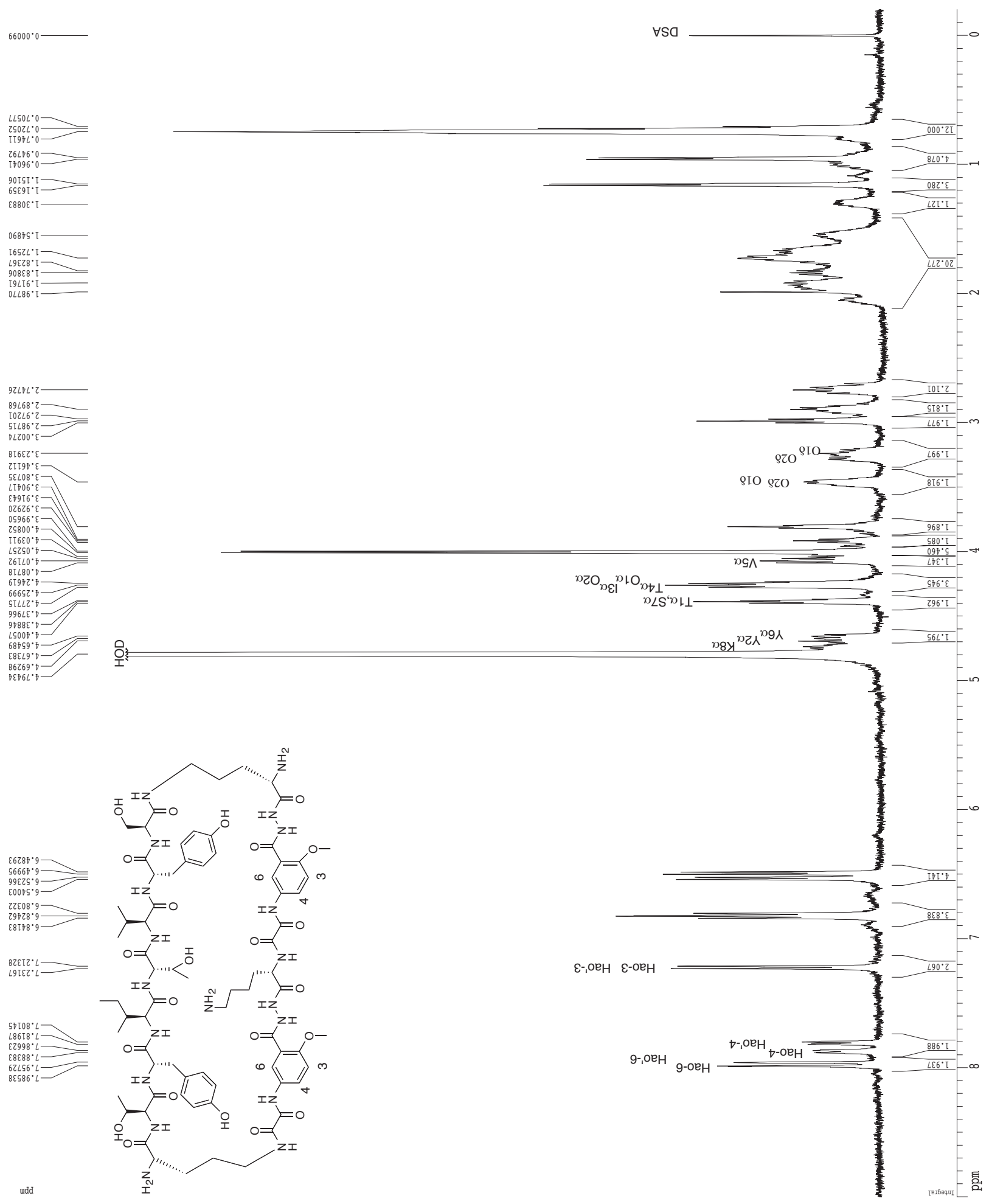


TOCSY spectrum of the monomer of peptide $\mathbf{3} \mathbf{j}$ $500 \mathrm{MHz}$ Cryoprobe (150-ms mixing time)

$0.4 \mathrm{mM}$ in $\mathrm{D}_{2} \mathrm{O}, 298 \mathrm{~K}$

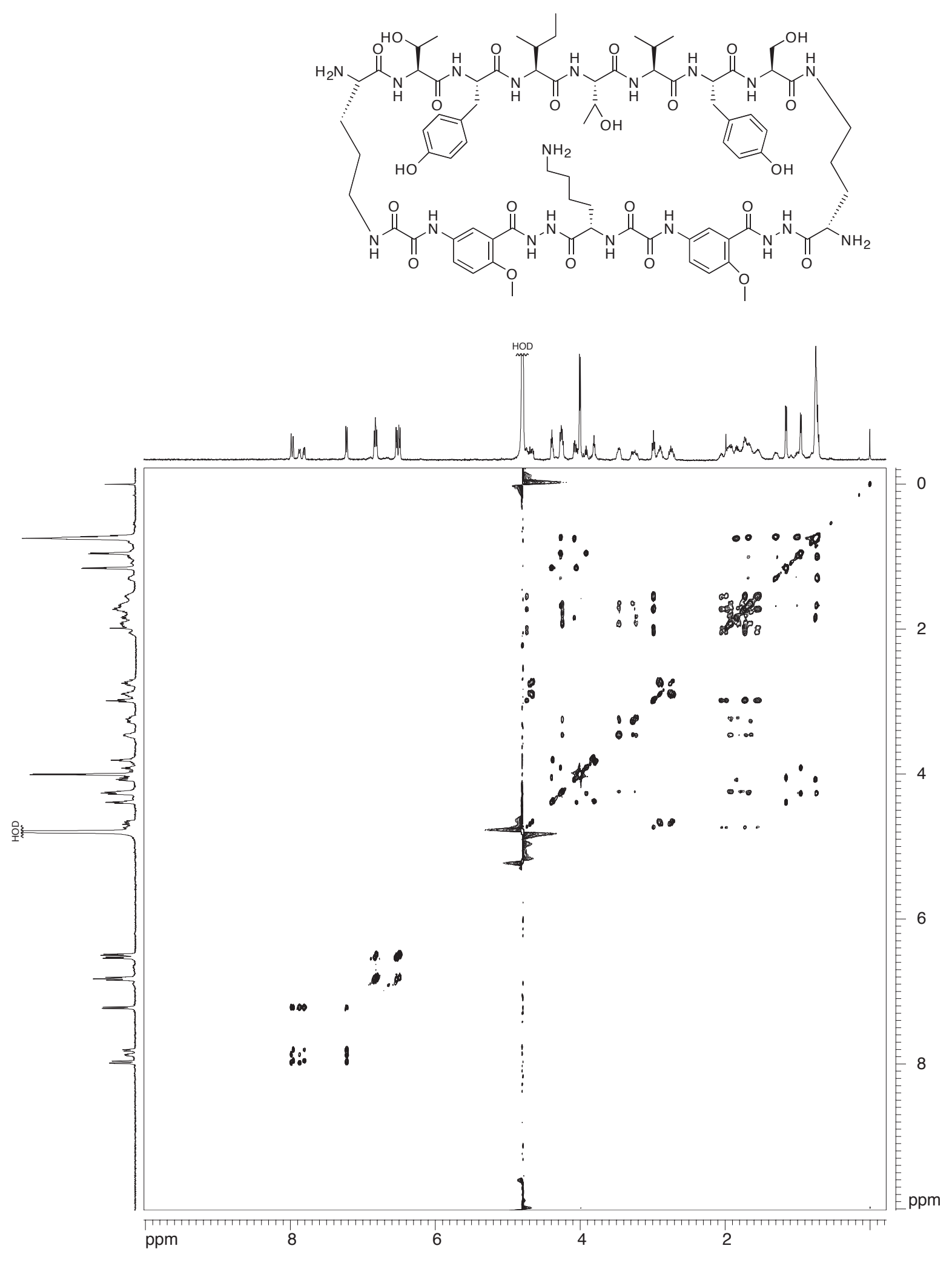


ROESY spectrum of the monomer of peptide $\mathbf{3} \mathbf{j}$ $500 \mathrm{MHz}$ Cryoprobe (300-ms mixing time)

$0.4 \mathrm{mM}$ in $\mathrm{D}_{2} \mathrm{O}, 298 \mathrm{~K}$

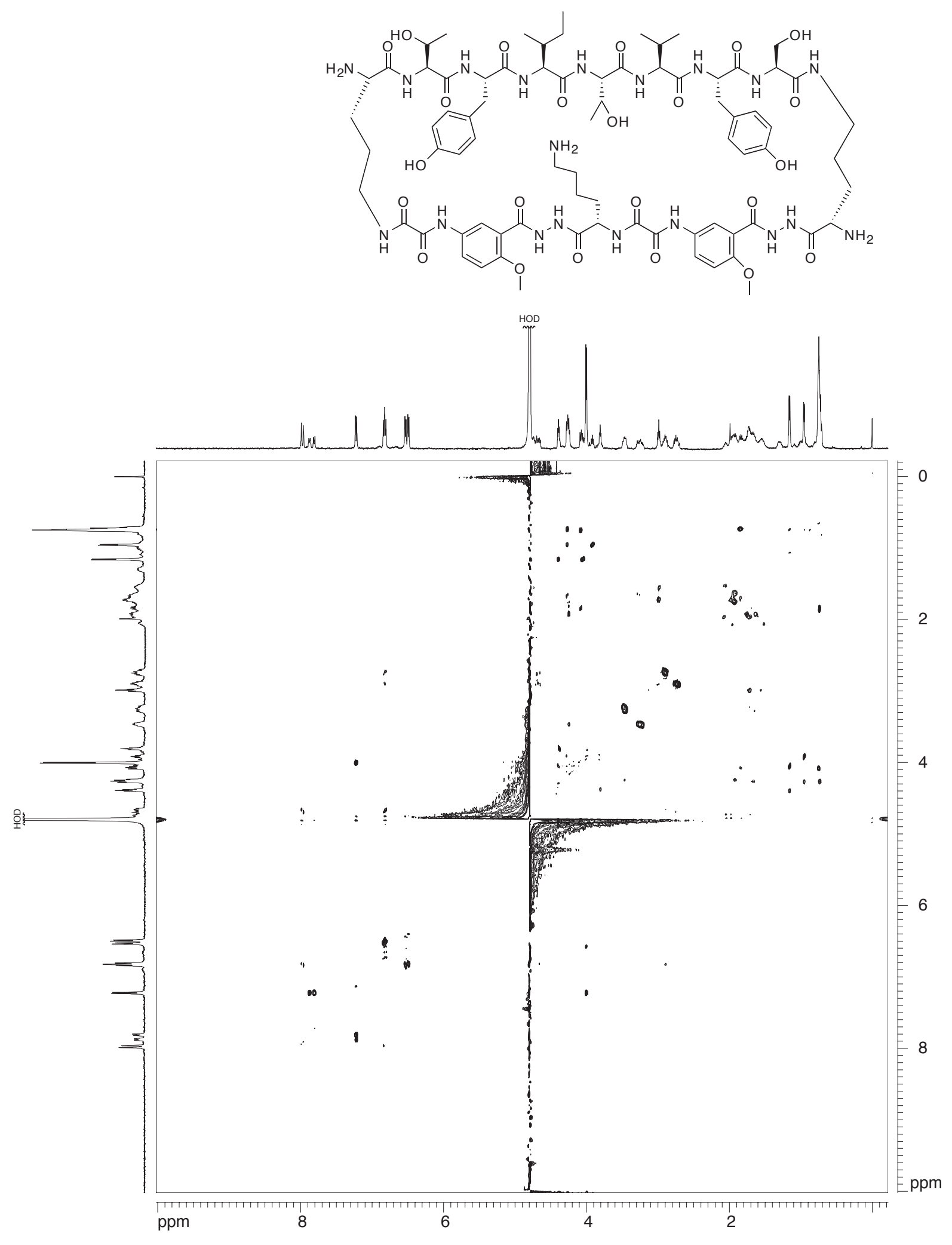


Overlay of $1 \mathrm{D}{ }^{1} \mathrm{H}$ NMR spectra of dilute and concentrated solutions of peptide $\mathbf{3 j}$ $500 \mathrm{MHz}$

$\mathrm{D}_{2} \mathrm{O}, 298 \mathrm{~K}$
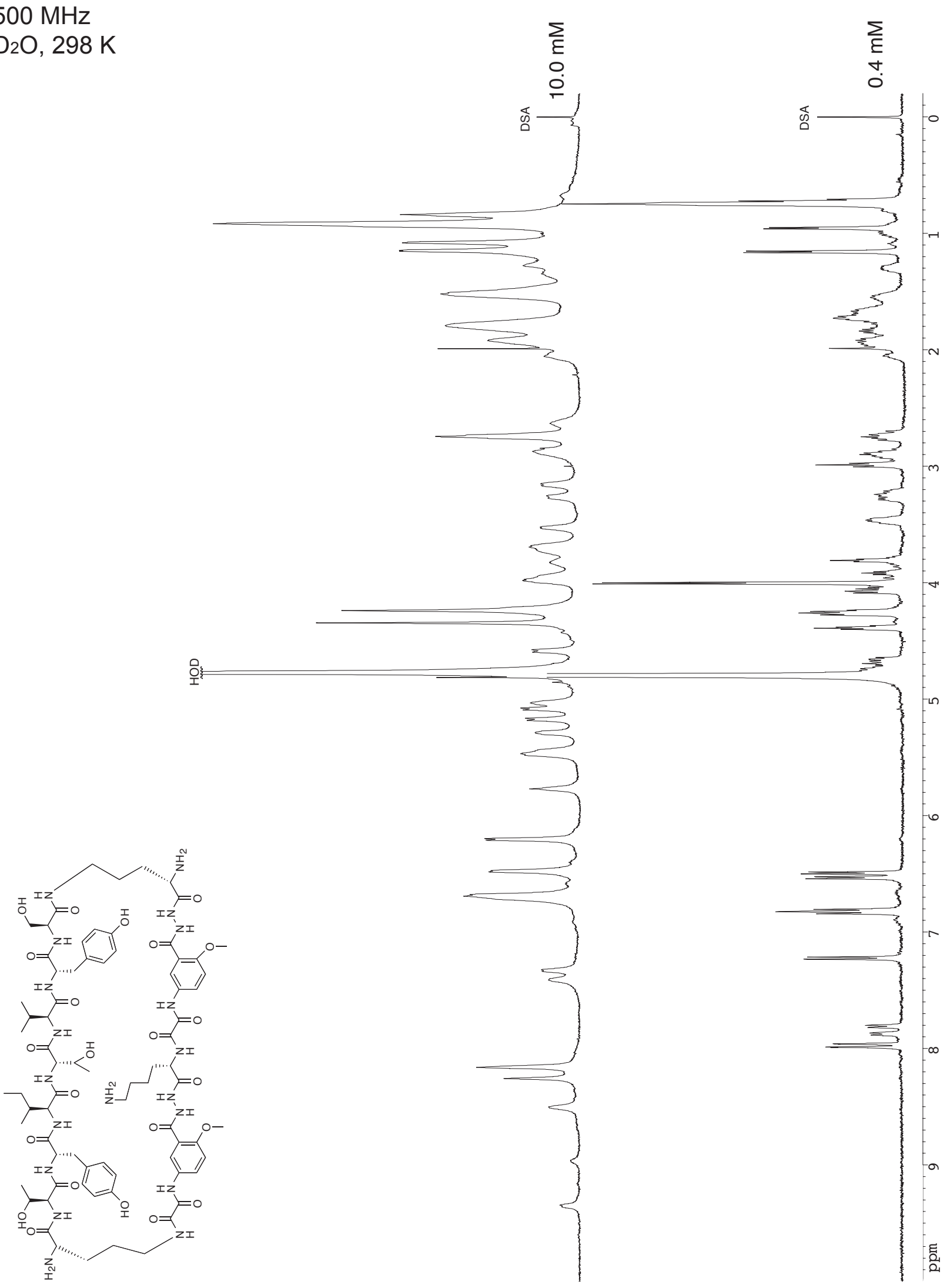
1D ${ }^{1} \mathrm{H}$ NMR spectrum of the monomer of peptide $3 \mathbf{k}$

$500 \mathrm{MHz}$ Cryoprobe

$0.4 \mathrm{mM}$ in $\mathrm{D}_{2} \mathrm{O}, 298 \mathrm{~K}$

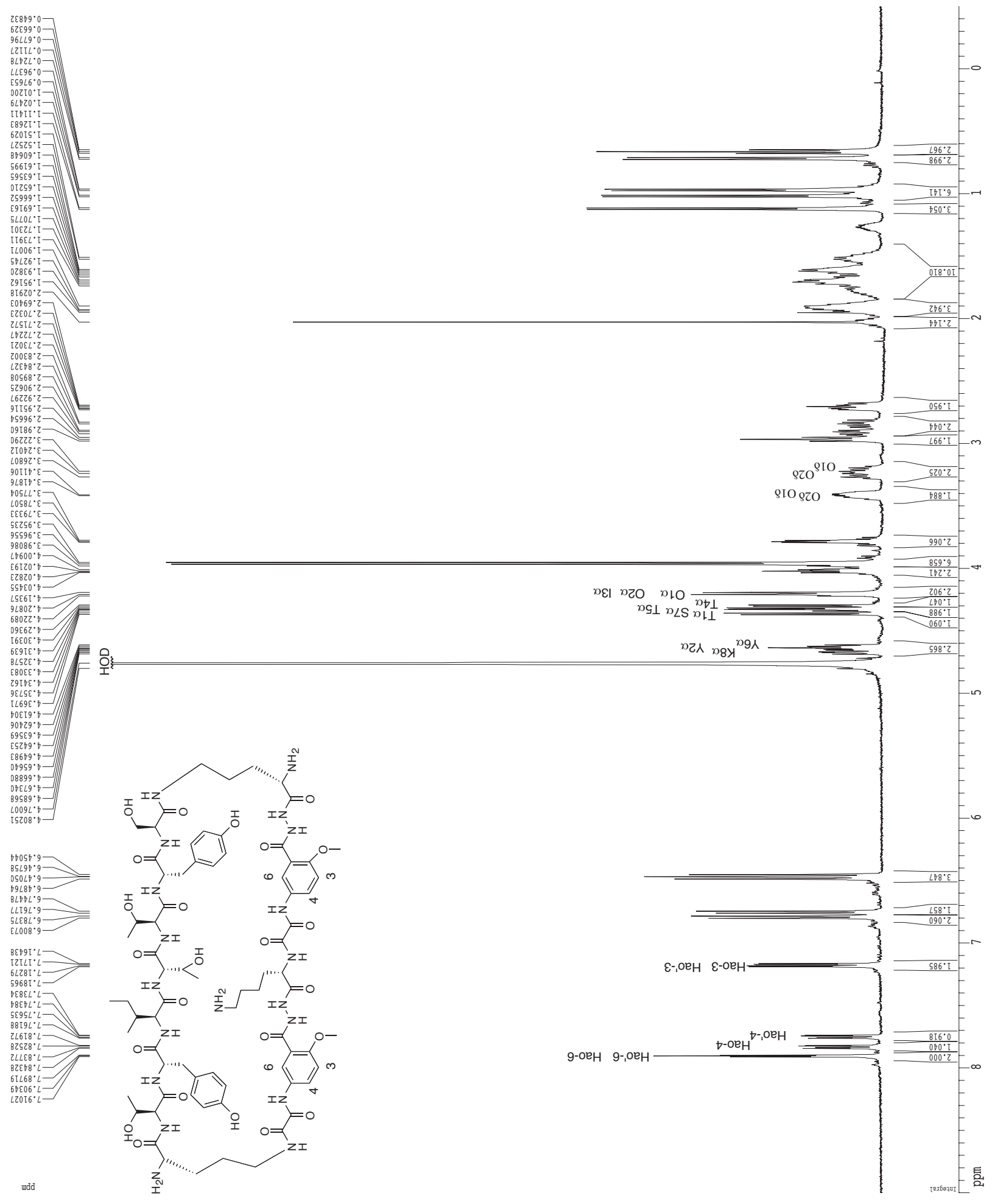


TOCSY spectrum of the monomer of peptide $\mathbf{3 k}$ $500 \mathrm{MHz}$ Cryoprobe (150-ms mixing time)

$0.4 \mathrm{mM}$ in $\mathrm{D}_{2} \mathrm{O}, 298 \mathrm{~K}$

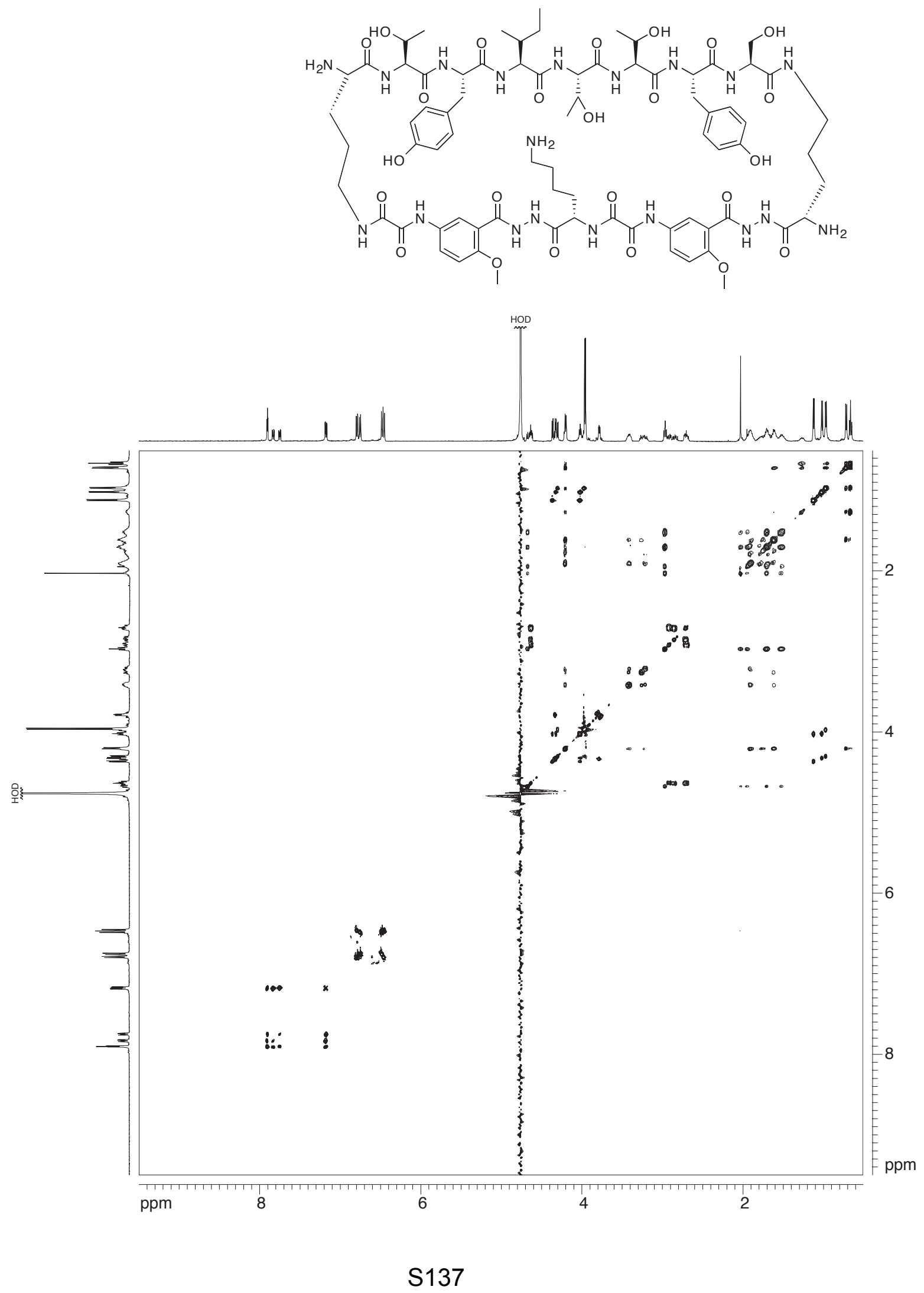


ROESY spectrum of the monomer of peptide $\mathbf{3 k}$ $500 \mathrm{MHz}$ Cryoprobe (300-ms mixing time)

$0.4 \mathrm{mM}$ in $\mathrm{D}_{2} \mathrm{O}, 298 \mathrm{~K}$

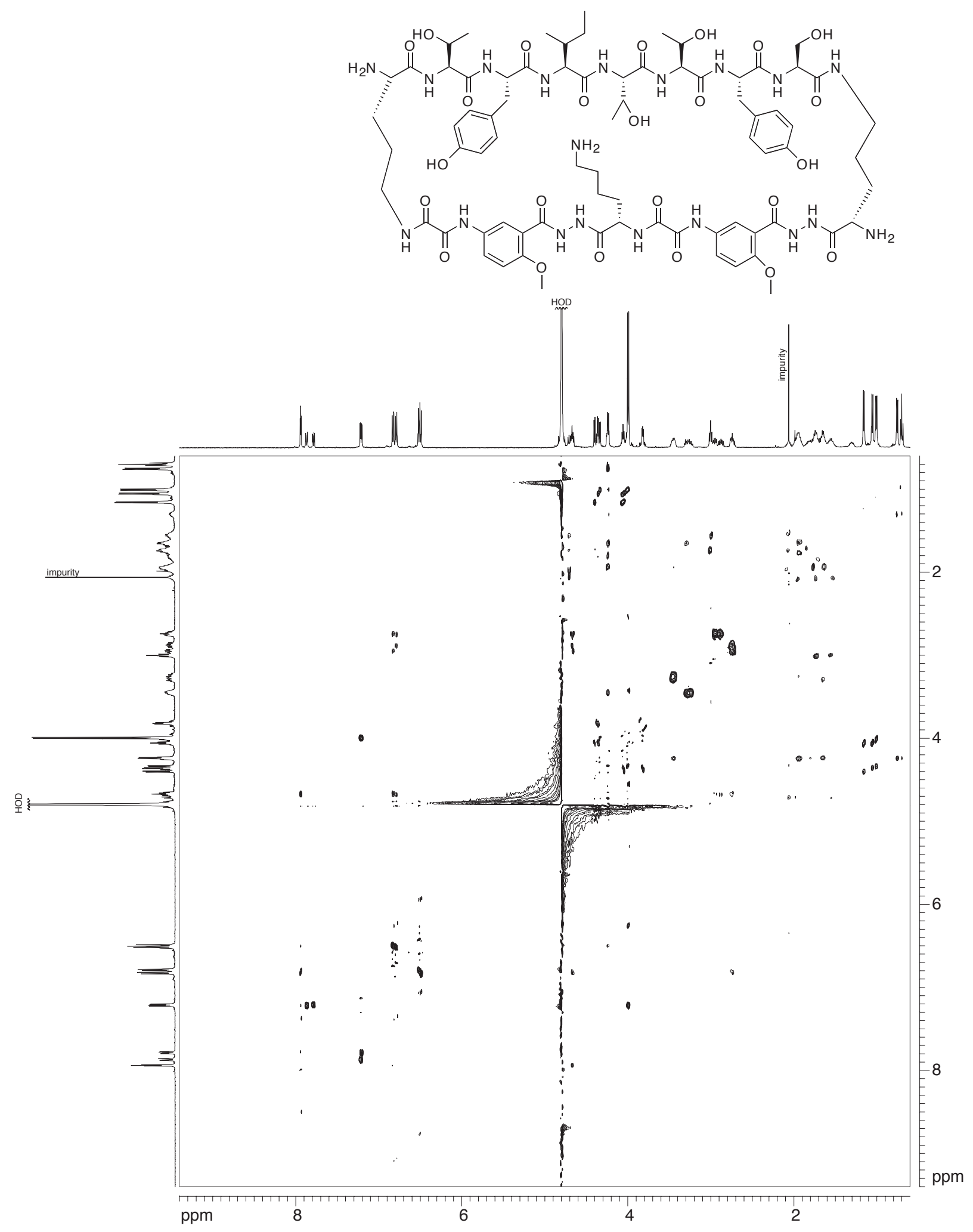


Overlay of $1 \mathrm{D}{ }^{1} \mathrm{H}$ NMR spectra of dilute and concentrated solutions of peptide $\mathbf{3 k}$ $500 \mathrm{MHz}$

$\mathrm{D}_{2} \mathrm{O}, 298 \mathrm{~K}$

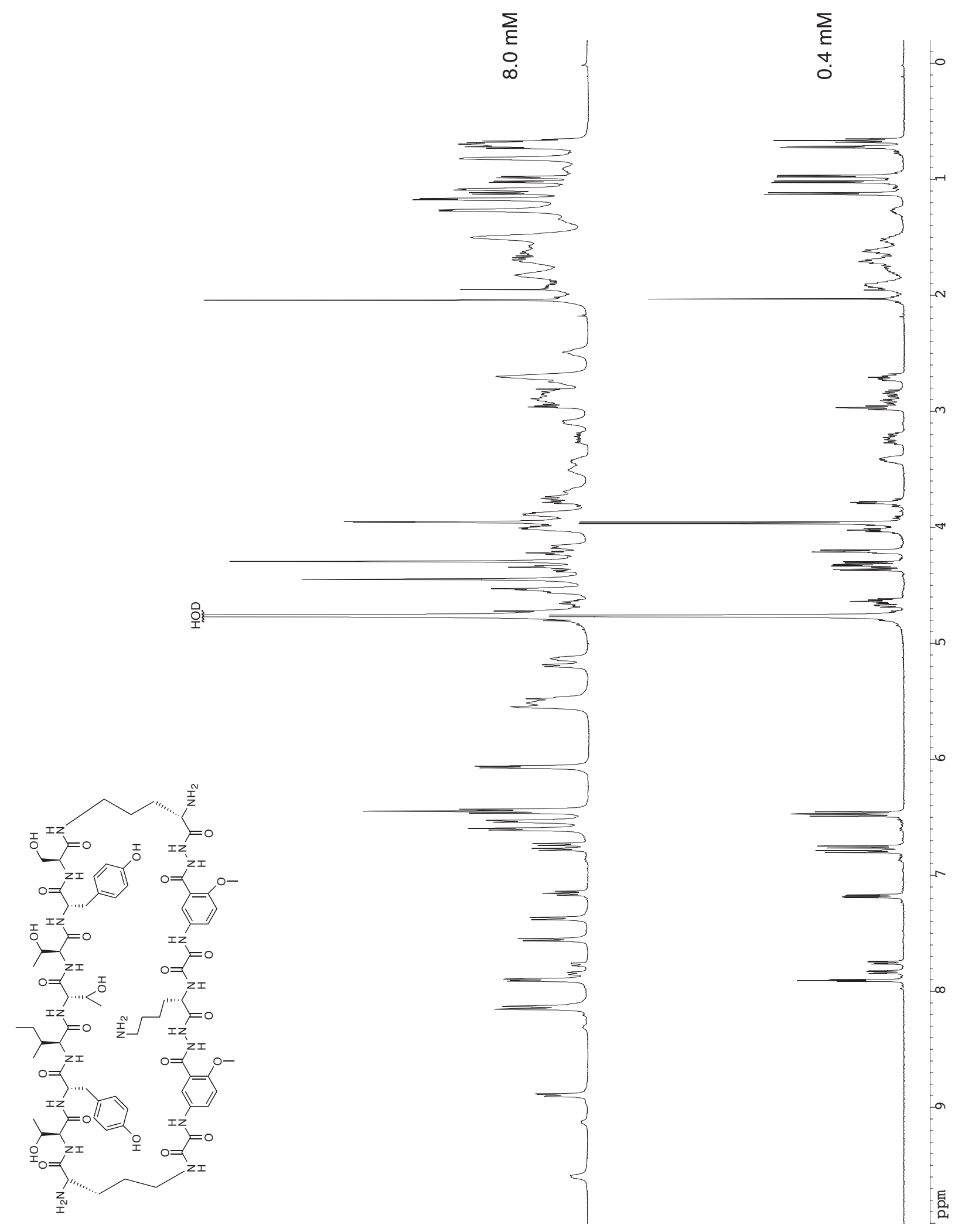




\section{D ${ }^{1} \mathrm{H}$ NMR spectrum of peptide $3 \mathbf{I}$}

\section{$500 \mathrm{MHz}$}

\section{$1.2 \mathrm{mM}$ in $\mathrm{D}_{2} \mathrm{O}, 298 \mathrm{~K}$}
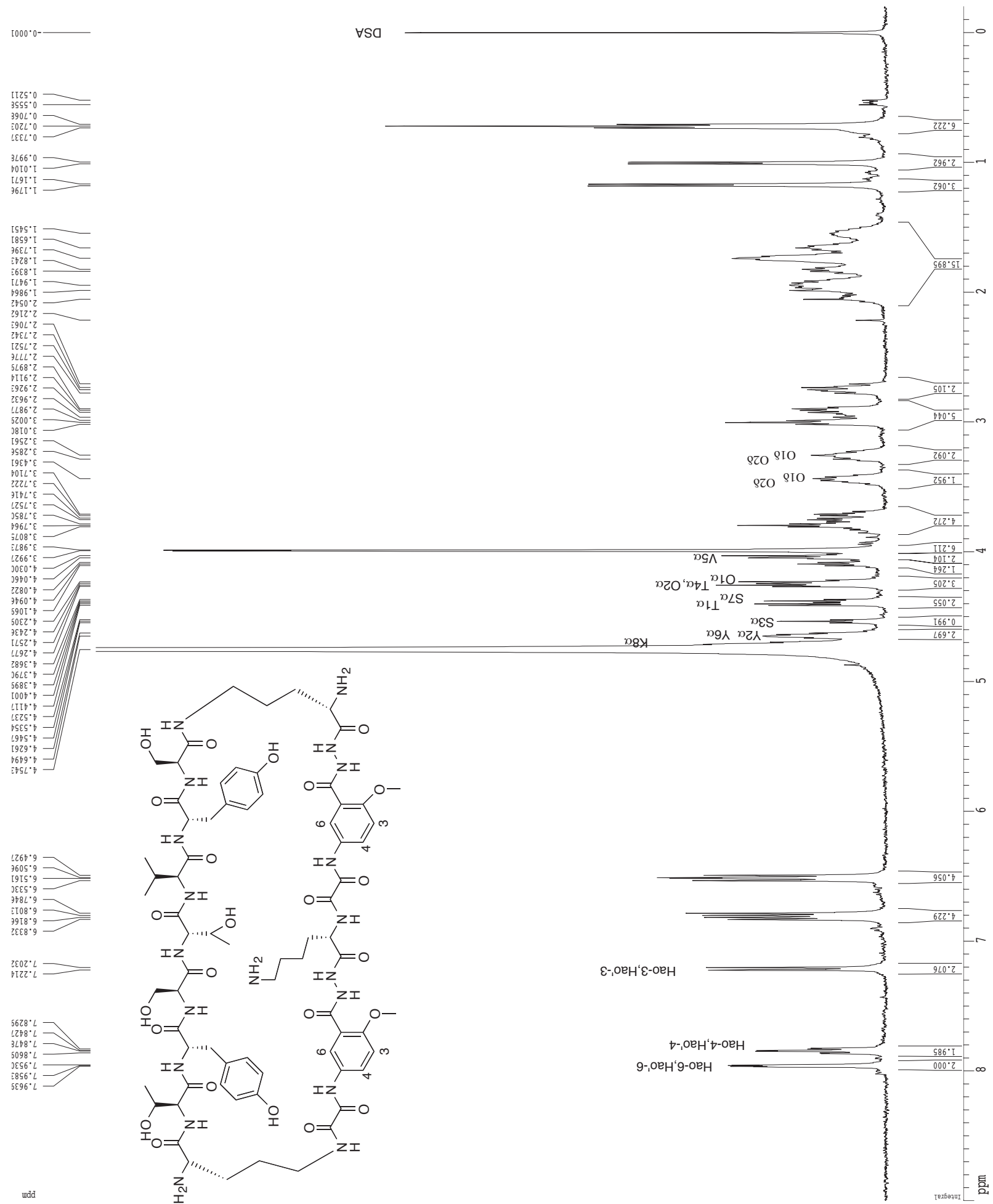
ROESY spectrum of peptide $3 \mathbf{I}$

$500 \mathrm{MHz}$ (150-ms mixing time)

$1.2 \mathrm{mM}$ in $\mathrm{D}_{2} \mathrm{O}, 298 \mathrm{~K}$

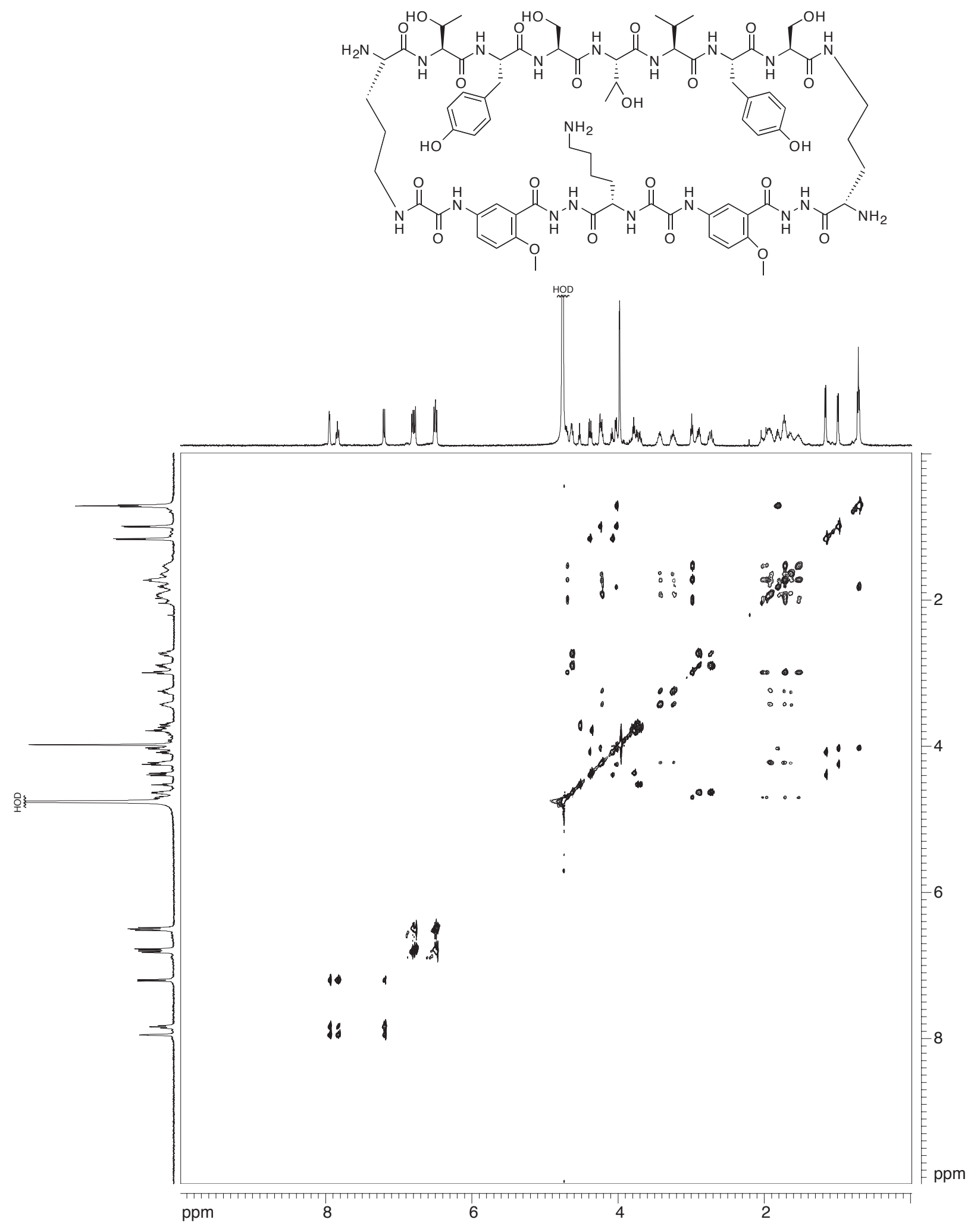


ROESY spectrum of peptide $\mathbf{3 l}$

$500 \mathrm{MHz}$ (300-ms mixing time)

$1.2 \mathrm{mM}$ in $\mathrm{D}_{2} \mathrm{O}, 298 \mathrm{~K}$

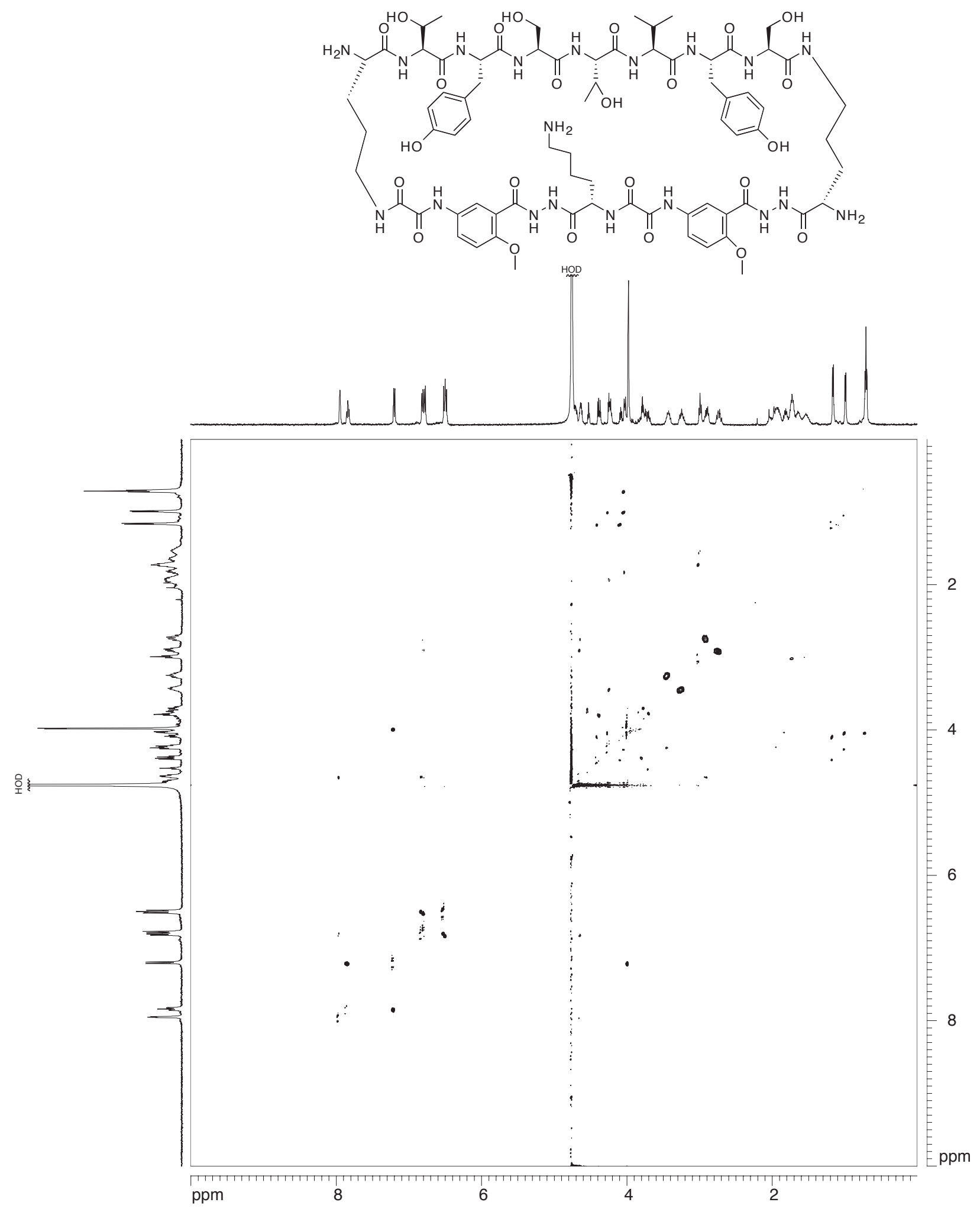


Overlay of $1 \mathrm{D}{ }^{1} \mathrm{H}$ NMR spectra of dilute and concentrated solutions of peptide 3 I $500 \mathrm{MHz}$

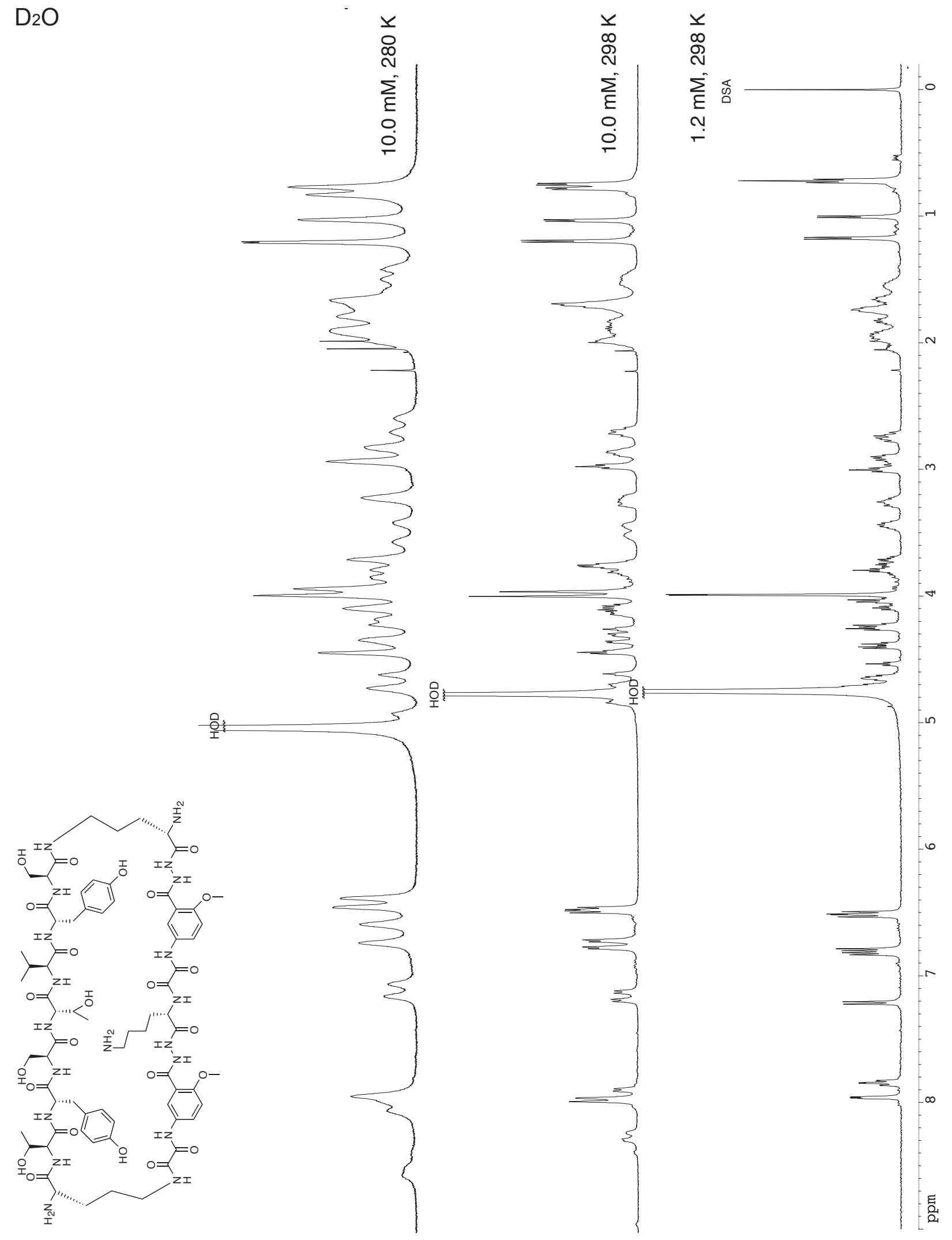


1D ${ }^{1} \mathrm{H}$ NMR spectrum of peptide $3 \mathrm{~m}$

$500 \mathrm{MHz}$

$1.0 \mathrm{mM}$ in $\mathrm{D}_{2} \mathrm{O}, 298 \mathrm{~K}$

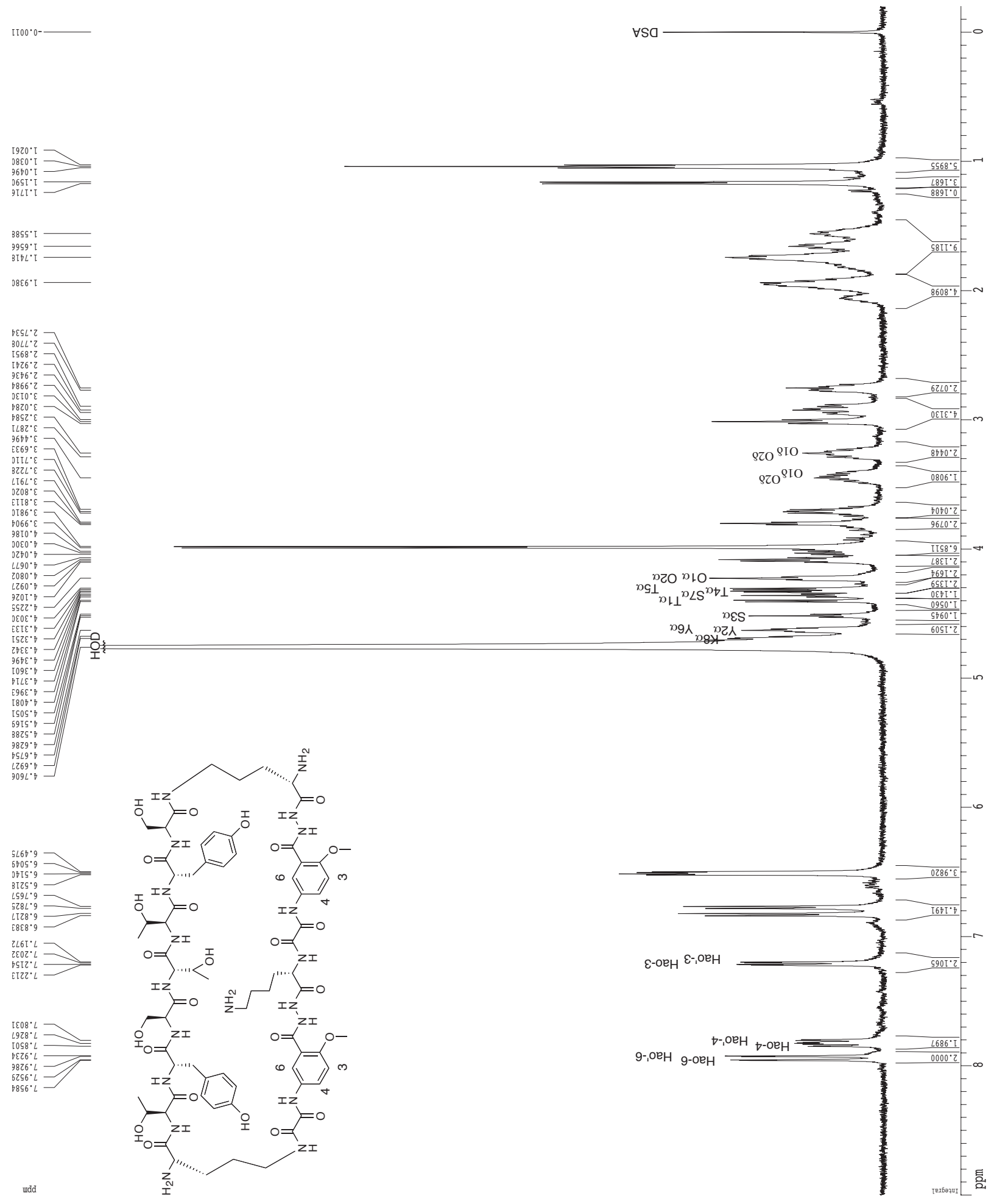


ROESY spectrum of peptide $3 \mathrm{~m}$

$500 \mathrm{MHz}$ (150-ms mixing time)

$1.0 \mathrm{mM}$ in $\mathrm{D}_{2} \mathrm{O}, 298 \mathrm{~K}$

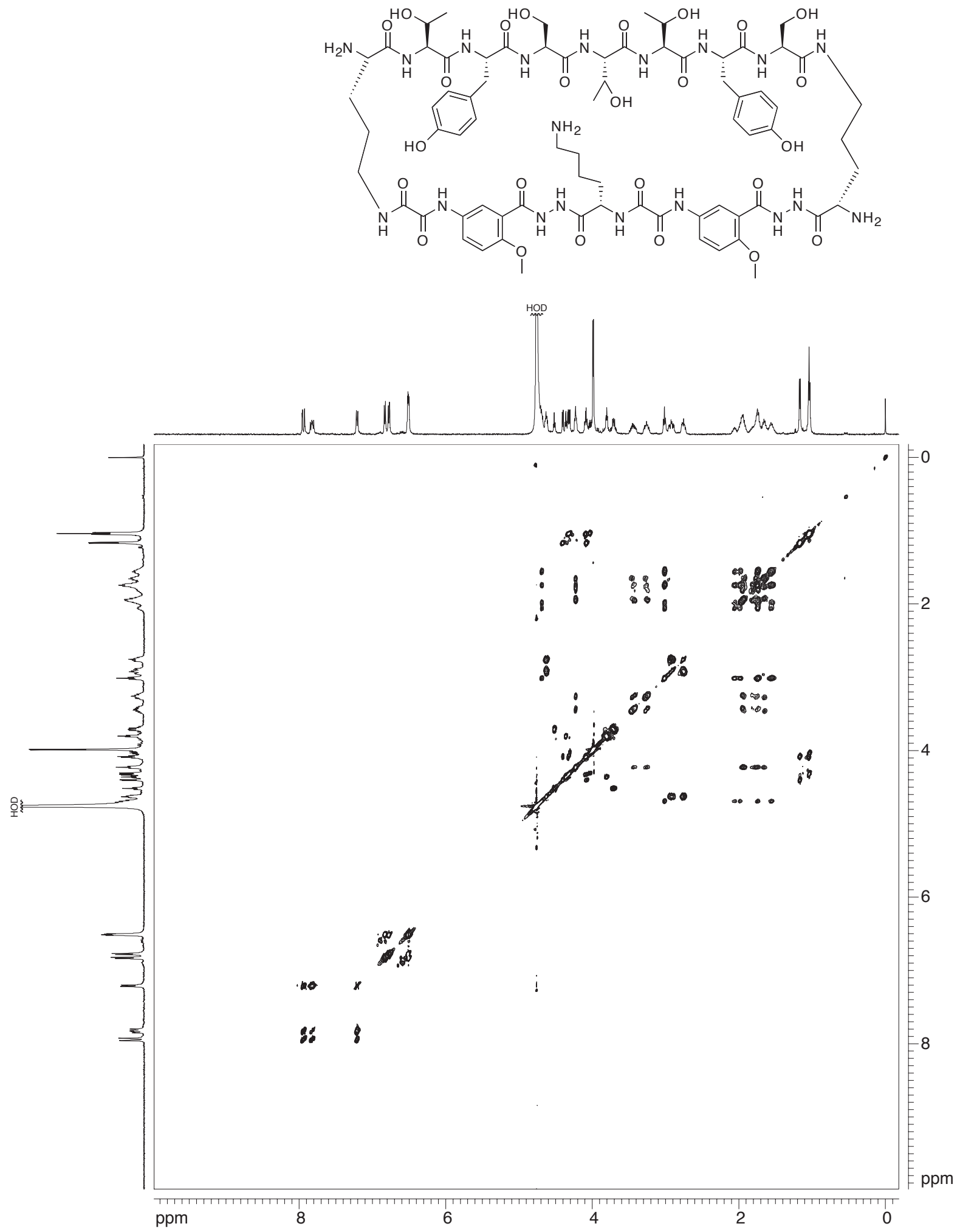


ROESY spectrum of peptide $3 \mathrm{~m}$

$500 \mathrm{MHz}$ (300-ms mixing time)

$1.0 \mathrm{mM}$ in $\mathrm{D}_{2} \mathrm{O}, 298 \mathrm{~K}$

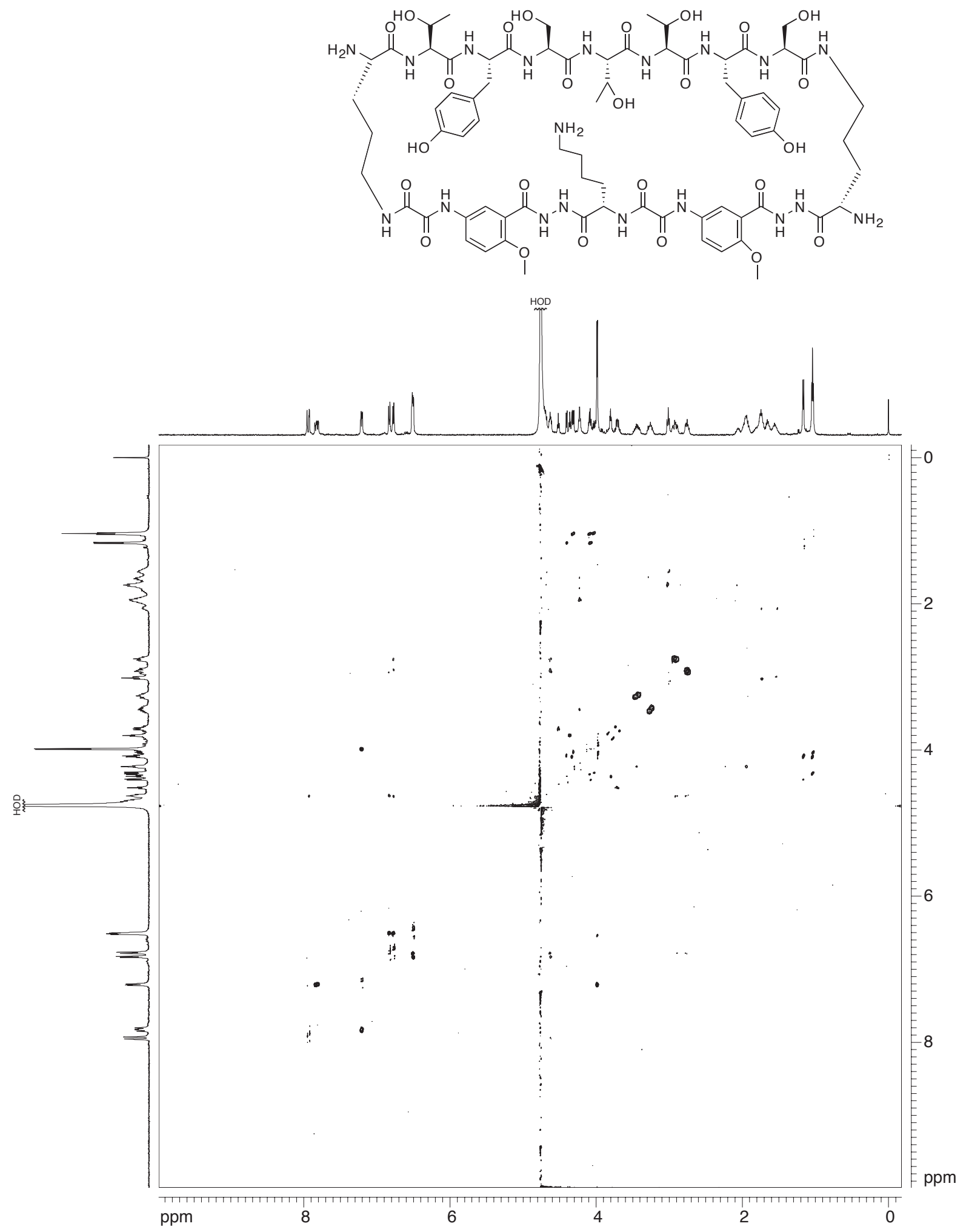


Overlay of $1 \mathrm{D}{ }^{1} \mathrm{H}$ NMR spectra of dilute and concentrated solutions of peptide $3 \mathrm{~m}$ $500 \mathrm{MHz}$

$\mathrm{D}_{2} \mathrm{O}$
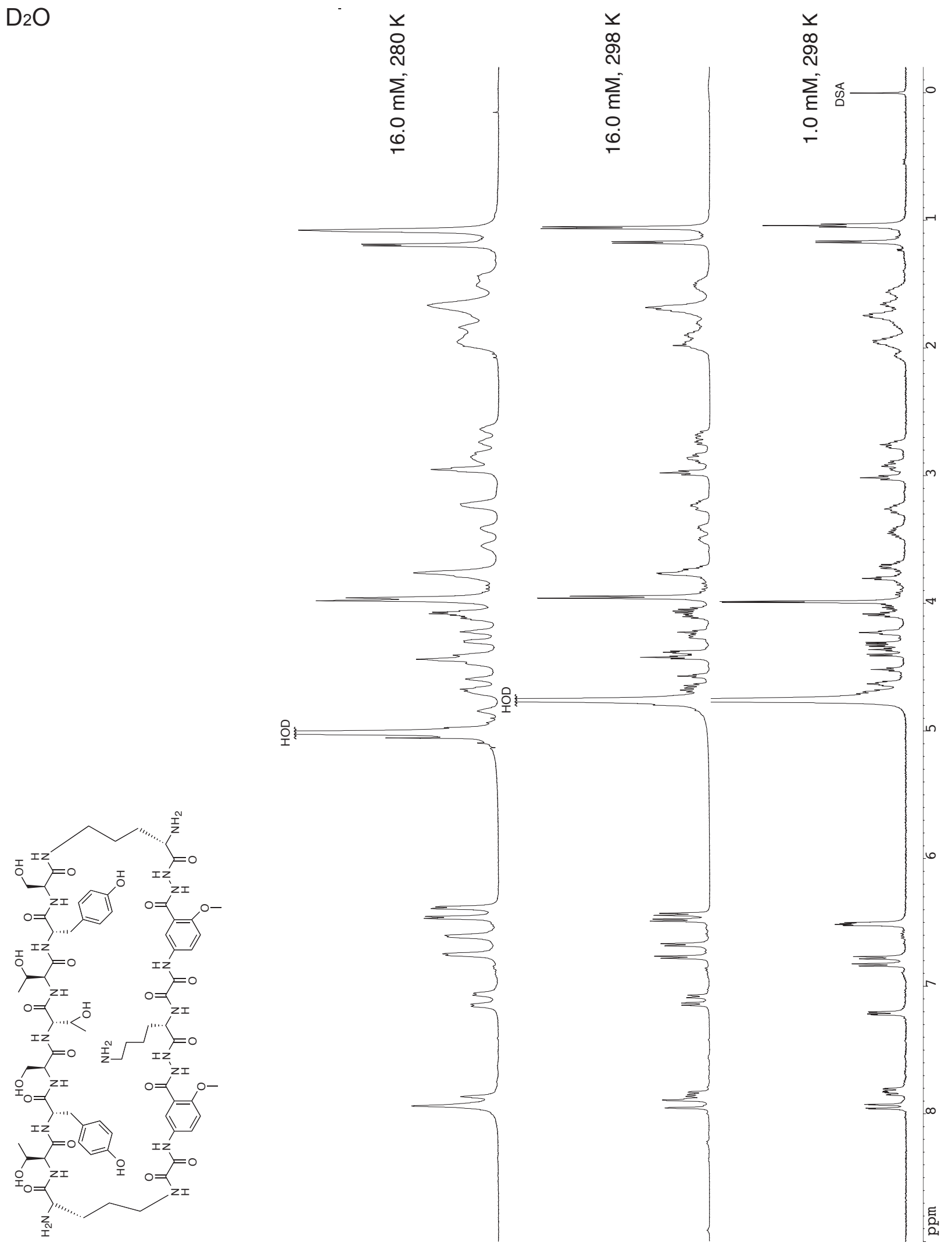
1D ${ }^{1} \mathrm{H}$ NMR spectrum of peptide $3 \mathrm{n}$

$500 \mathrm{MHz}$ Cryo probe

$0.8 \mathrm{mM}$ in $\mathrm{D}_{2} \mathrm{O}, 298 \mathrm{~K}$
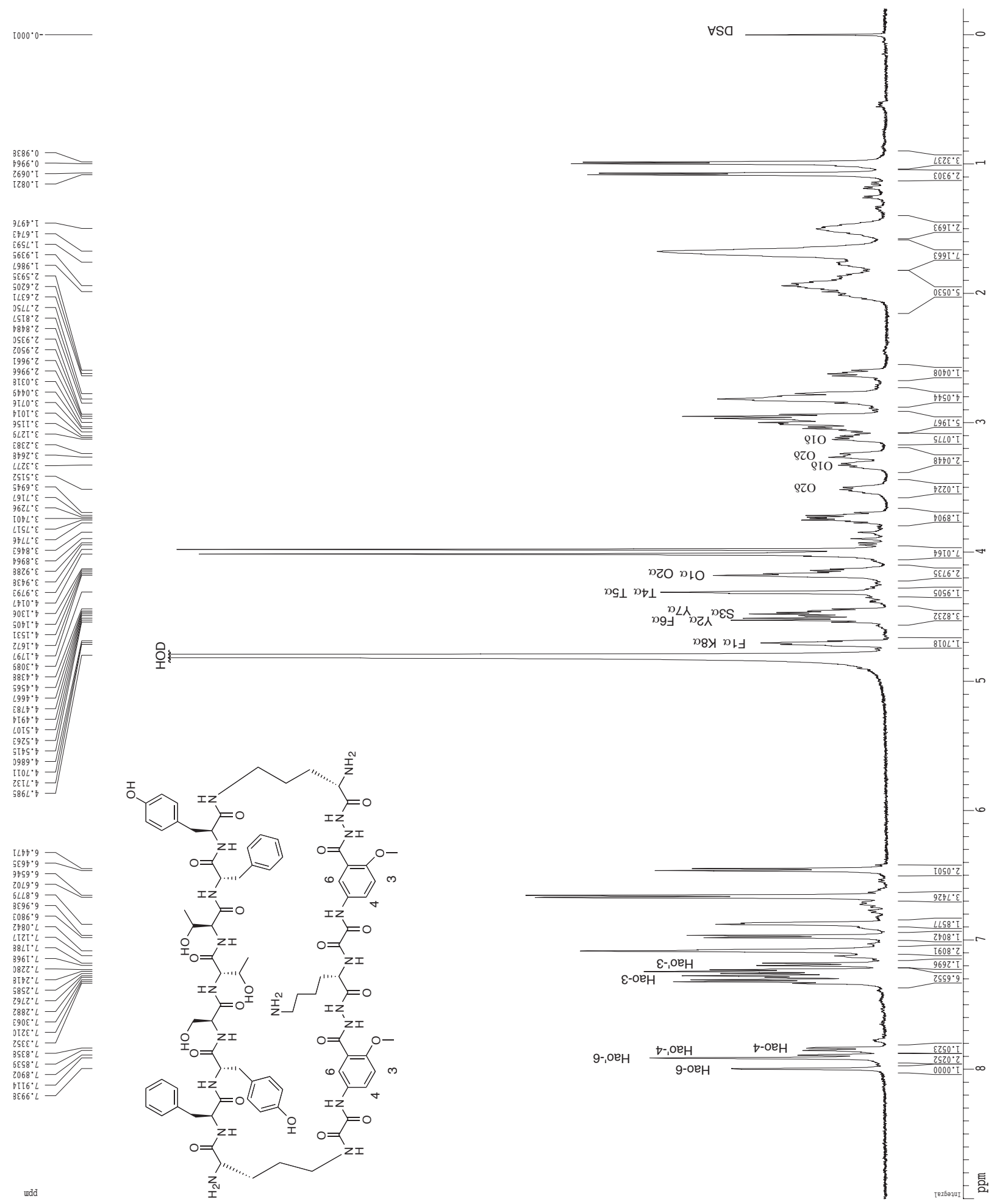
ROESY spectrum of peptide $3 \mathbf{n}$

$500 \mathrm{MHz}$ Cryoprobe (150-ms mixing time)

$0.5 \mathrm{mM}$ in $\mathrm{D}_{2} \mathrm{O}, 298 \mathrm{~K}$
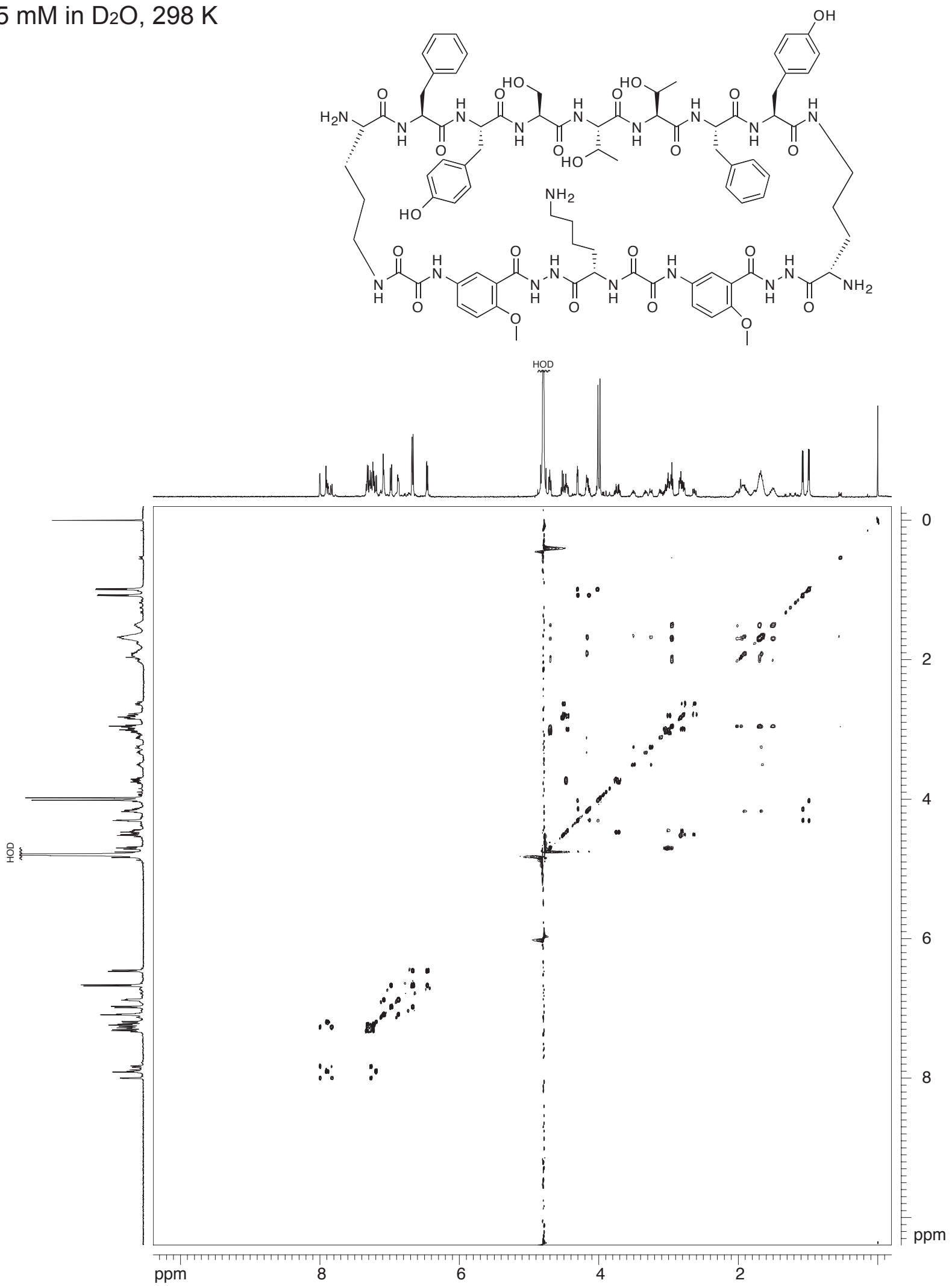
ROESY spectrum of peptide $3 \mathbf{n}$

$500 \mathrm{MHz}$ Cryoprobe (300-ms mixing time)

$0.5 \mathrm{mM}$ in $\mathrm{D}_{2} \mathrm{O}, 298 \mathrm{~K}$

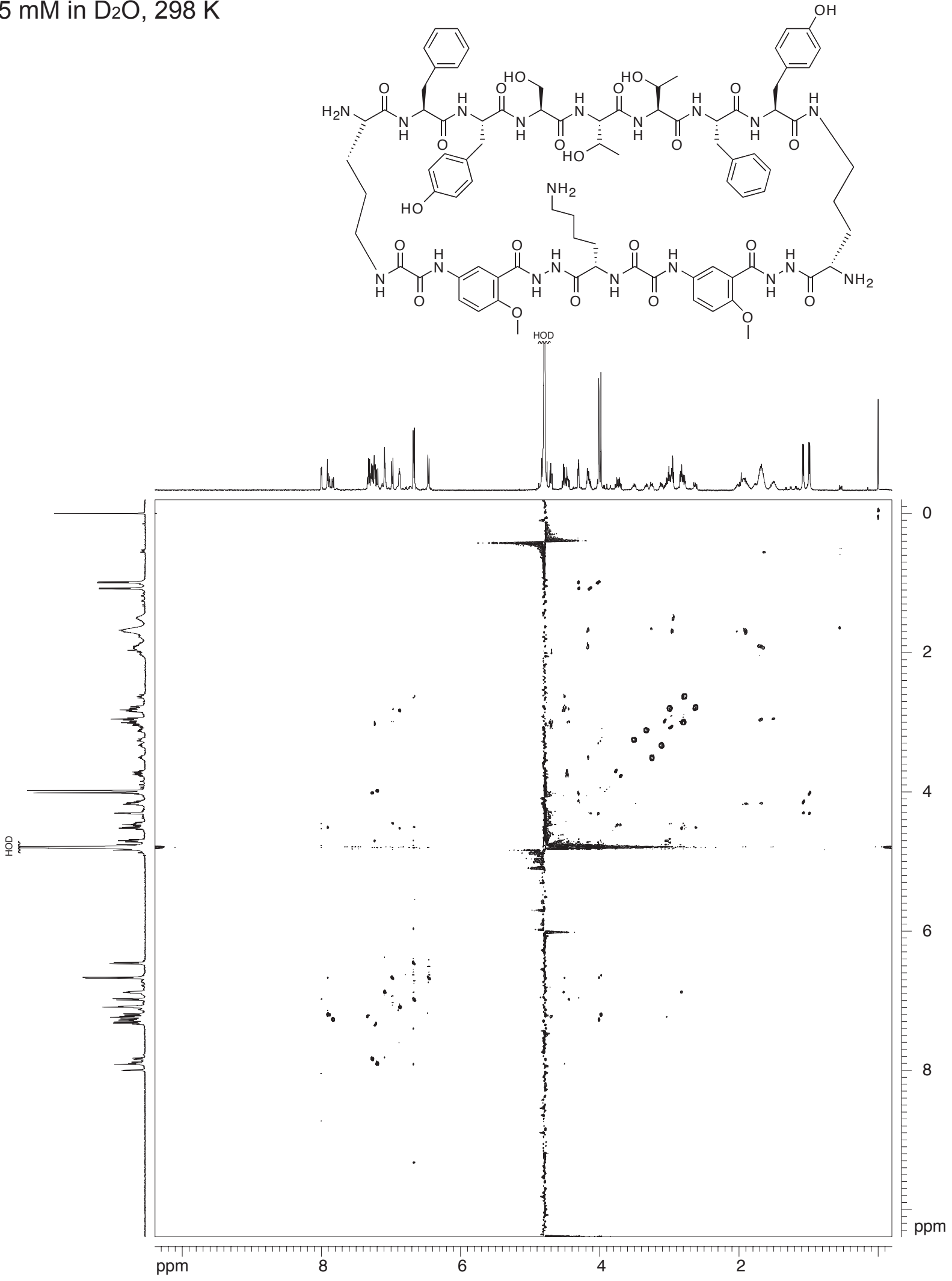


Overlay of $1 \mathrm{D}{ }^{1} \mathrm{H}$ NMR spectra of dilute and concentrated solutions of peptide $3 \mathbf{n}$ $500 \mathrm{MHz}$

$\mathrm{D}_{2} \mathrm{O}$
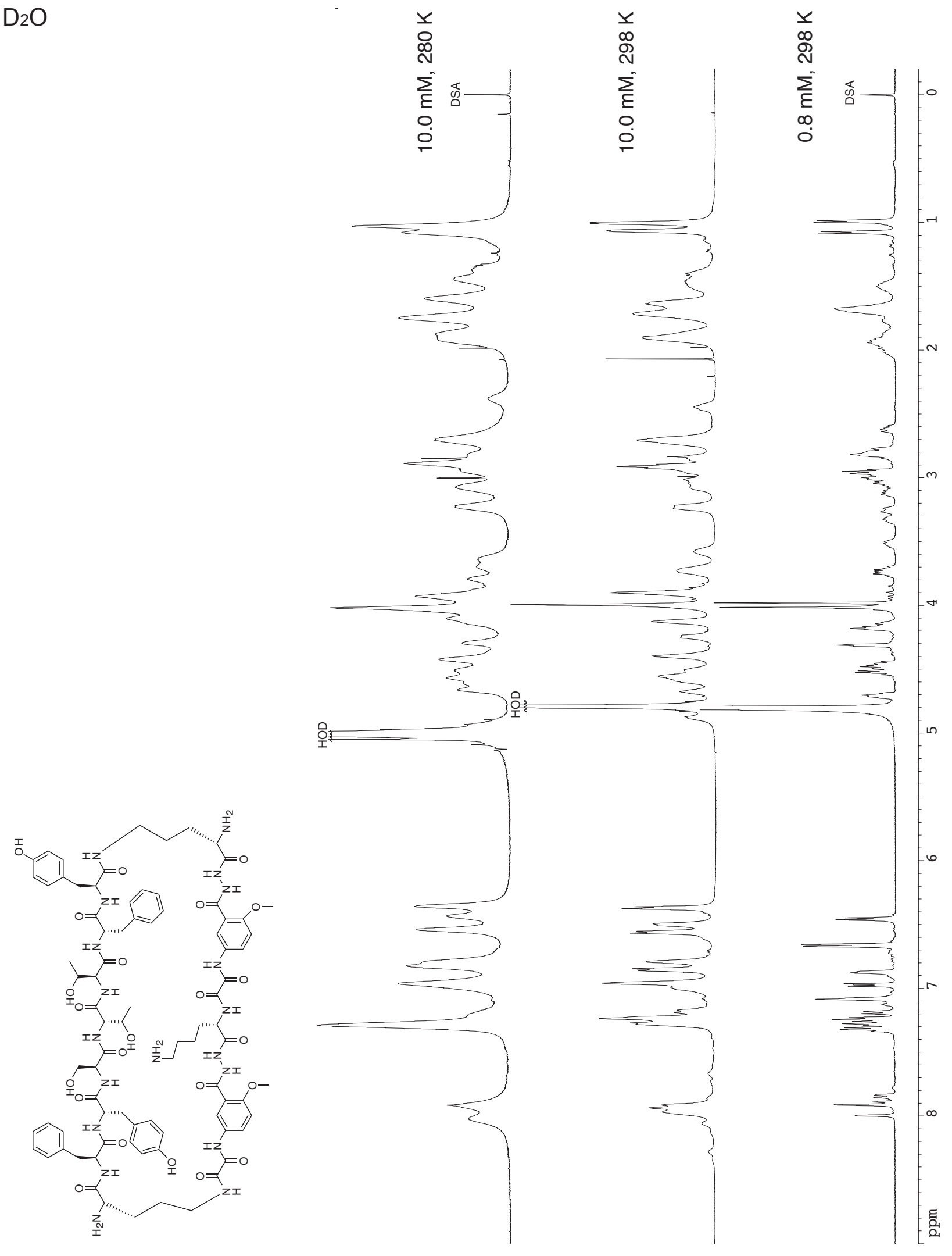
$\alpha$-Proton chemical shifts of the monomers of peptides $3 a-3 n$.

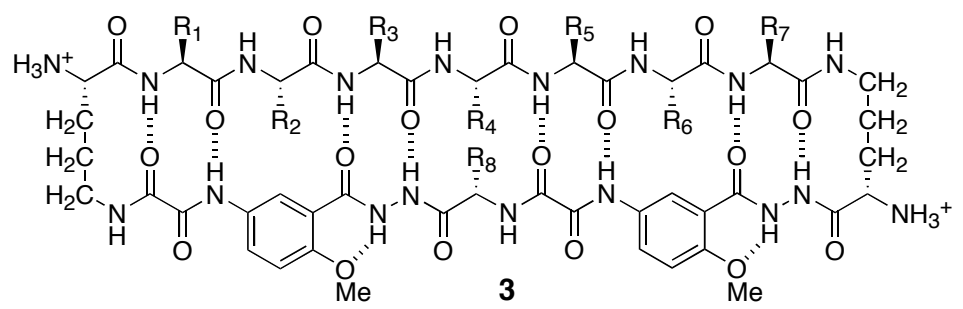

${ }^{1} \mathrm{H}$ NMR chemical shifts (ppm) at $0.4-1.5 \mathrm{mM} \mathrm{D}_{2} \mathrm{O}$ solution of peptide at $298 \mathrm{~K}$ measured against DSA $(\delta=0 \mathrm{ppm})$

\begin{tabular}{|c|c|c|c|c|c|c|c|c|}
\hline Peptide & $\mathbf{R}_{\mathbf{1}}$ & $\mathbf{R}_{\mathbf{2}}$ & $\mathbf{R}_{\mathbf{3}}$ & $\mathbf{R}_{\mathbf{4}}$ & $\mathbf{R}_{\mathbf{5}}$ & $\mathbf{R}_{\mathbf{6}}$ & $\mathbf{R}_{\mathbf{7}}$ & $\mathbf{R}_{\mathbf{8}}$ \\
\hline $\mathbf{3 a}$ & 4.43 & 4.51 & 4.61 & 4.29 & 4.62 & 4.40 & 4.35 & 4.65 \\
\hline $\mathbf{3 b}$ & 4.43 & 4.51 & 4.53 & 4.28 & 4.59 & 4.39 & 4.35 & 4.64 \\
\hline $\mathbf{3 c}$ & 4.43 & 4.52 & 4.55 & 4.29 & 4.62 & 4.41 & 4.37 & 4.67 \\
\hline $\mathbf{3 d}$ & 4.48 & 4.60 & 4.67 & 4.57 & 4.61 & 4.46 & 4.38 & 4.63 \\
\hline $\mathbf{3 e}$ & 4.35 & 4.38 & 4.62 & 4.30 & 4.55 & 4.10 & 4.32 & 4.63 \\
\hline $\mathbf{3 f}$ & 4.49 & 5.23 & 5.05 & 4.89 & 5.03 & 4.75 & 4.51 & 5.04 \\
\hline $\mathbf{3 g}$ & 4.47 & 5.23 & 5.05 & 4.89 & 5.03 & 4.70 & 4.49 & 5.04 \\
\hline $\mathbf{3 h}$ & 4.45 & 4.44 & 4.56 & 4.24 & 4.58 & 4.31 & 4.18 & 4.62 \\
\hline $\mathbf{3 i}$ & 4.43 & 4.45 & 4.37 & 4.27 & 4.11 & 4.38 & 4.37 & 4.62 \\
\hline $\mathbf{3 j}$ & 4.40 & 4.69 & 4.27 & 4.27 & 4.08 & 4.67 & 4.38 & 4.75 \\
\hline $\mathbf{3 k}$ & 4.40 & 4.65 & 4.25 & 4.33 & 4.35 & 4.67 & 4.37 & 4.70 \\
\hline $\mathbf{3 \mathbf { l }}$ & 4.41 & 4.65 & 4.54 & 4.26 & 4.04 & 4.64 & 4.38 & 4.70 \\
\hline $\mathbf{3 m}$ & 4.40 & 4.64 & 4.52 & 4.33 & 4.31 & 4.62 & 4.36 & 4.69 \\
\hline $\mathbf{3 n}$ & 4.70 & 4.51 & 4.47 & 4.31 & 4.30 & 4.52 & 4.45 & 4.70 \\
\hline
\end{tabular}

$\Delta \delta \mathrm{H}_{\alpha}=\left[\delta \mathrm{H}_{\alpha}(\right.$ observed $\left.)-\delta \mathrm{H}_{\alpha}(\text { random coil })^{23}\right](\mathrm{ppm})$

\begin{tabular}{|c|c|c|c|c|c|c|c|c|}
\hline Peptide & $\mathbf{R}_{\mathbf{1}}$ & $\mathbf{R}_{\mathbf{2}}$ & $\mathbf{R}_{\mathbf{3}}$ & $\mathbf{R}_{\mathbf{4}}$ & $\mathbf{R}_{\mathbf{5}}$ & $\mathbf{R}_{\mathbf{6}}$ & $\mathbf{R}_{\mathbf{7}}$ & $\mathbf{R}_{\mathbf{8}}$ \\
\hline $\mathbf{3 a}$ & 0.08 & 0.04 & -0.02 & -0.06 & 0.07 & 0.05 & -0.12 & 0.32 \\
\hline $\mathbf{3 b}$ & 0.08 & 0.04 & -0.10 & -0.04 & 0.04 & 0.04 & -0.12 & 0.31 \\
\hline $\mathbf{3 c}$ & 0.08 & 0.05 & -0.08 & -0.03 & 0.07 & 0.06 & -0.10 & 0.34 \\
\hline $\mathbf{3 d}$ & 0.13 & 0.13 & 0.04 & 0.02 & 0.06 & 0.11 & -0.09 & 0.30 \\
\hline $\mathbf{3 e}$ & 0.00 & 0.06 & -0.01 & -0.05 & 0.00 & -0.02 & -0.15 & 0.30 \\
\hline $\mathbf{3 f}$ & 0.14 & 0.68 & 0.42 & 0.54 & 0.48 & 0.12 & 0.04 & 0.71 \\
\hline $\mathbf{3 g}$ & 0.12 & 0.68 & 0.42 & 0.54 & 0.48 & 0.15 & 0.02 & 0.71 \\
\hline $\mathbf{3 h}$ & 0.12 & -0.03 & -0.07 & -0.10 & 0.03 & -0.04 & -0.15 & 0.29 \\
\hline $\mathbf{3 i}$ & 0.08 & -0.02 & 0.05 & -0.08 & -0.01 & 0.03 & -0.10 & 0.29 \\
\hline $\mathbf{3 j}$ & 0.05 & 0.14 & 0.10 & -0.08 & -0.04 & 0.12 & -0.09 & 0.42 \\
\hline $\mathbf{3 k}$ & 0.05 & 0.10 & 0.08 & -0.02 & 0.00 & 0.12 & -0.10 & 0.37 \\
\hline $\mathbf{3 l}$ & 0.06 & 0.10 & 0.07 & -0.09 & -0.08 & 0.09 & -0.09 & 0.37 \\
\hline $\mathbf{3 m}$ & 0.05 & 0.09 & 0.05 & -0.02 & -0.04 & 0.07 & -0.09 & 0.36 \\
\hline $\mathbf{3 n}$ & 0.07 & -0.04 & 0.00 & -0.04 & -0.05 & -0.11 & -0.10 & 0.37 \\
\hline
\end{tabular}

23 Wishart, D. S.; Sykes, B. D. Methods Enzymol. 1994, 239, 363-392. 
$\Delta \delta^{\delta}$ Orn Values for the monomers of peptides 3a-3n.
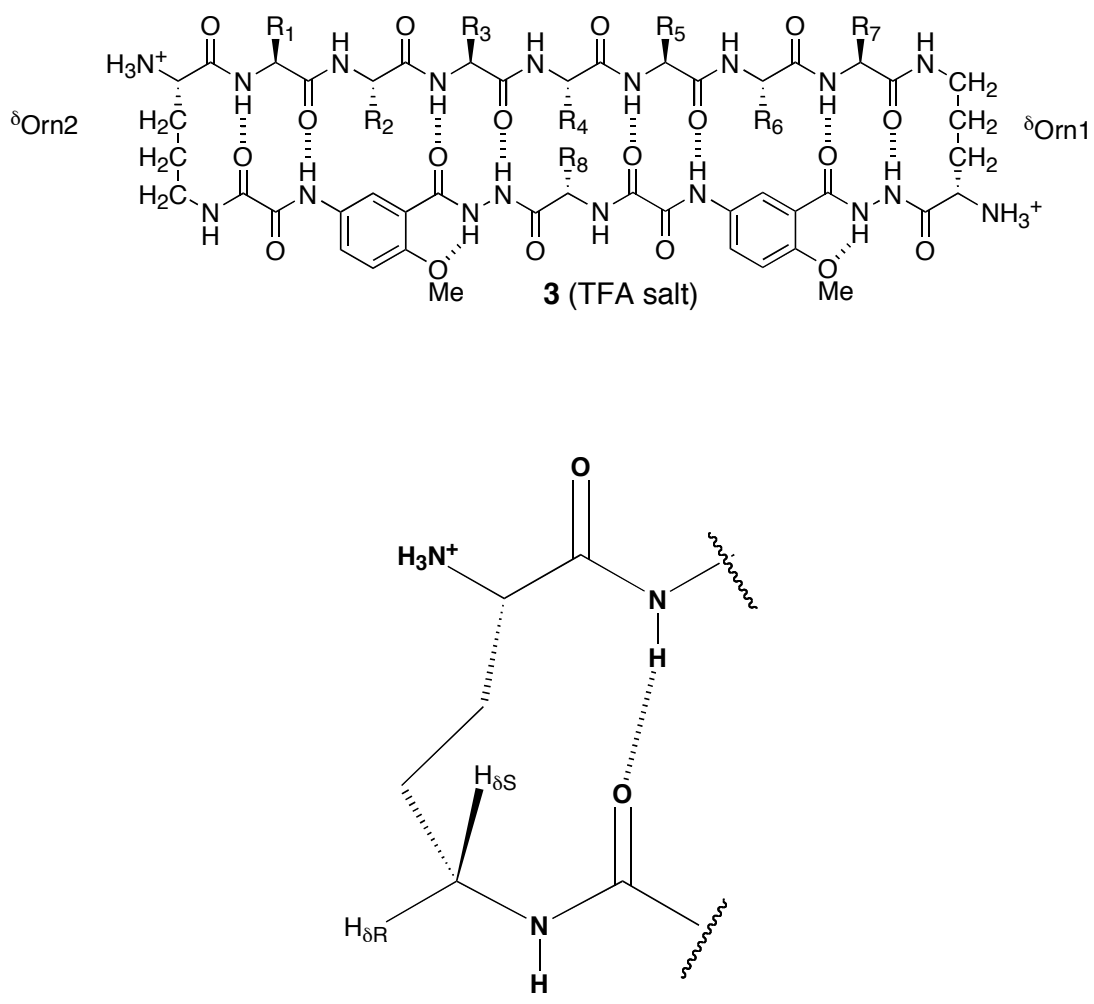

$\Delta \delta^{\delta}$ Orn $=\delta H_{\delta S}-\delta H_{\delta R}$ for ${ }^{\delta}$ Orn turn units (ppm)

\begin{tabular}{|c|c|c|}
\hline Peptide & $\Delta \boldsymbol{\delta}^{\boldsymbol{\delta}} \mathbf{O r n 1}$ & $\Delta \boldsymbol{\delta}^{\boldsymbol{\delta}} \mathbf{O r n 2}$ \\
\hline $\mathbf{3 a}$ & 0.16 & 0.15 \\
\hline $\mathbf{3 b}$ & 0.17 & 0.14 \\
\hline $\mathbf{3 c}$ & 0.15 & 0.11 \\
\hline $\mathbf{3 d}$ & 0.21 & 0.20 \\
\hline $\mathbf{3 e}$ & 0.29 & 0.15 \\
\hline $\mathbf{3 f}$ & 0.51 & 0.45 \\
\hline $\mathbf{3 g}$ & 0.50 & 0.45 \\
\hline $\mathbf{3 h}$ & 0.23 & 0.06 \\
\hline $\mathbf{3 i}$ & 0.09 & 0.04 \\
\hline $\mathbf{3 j}$ & 0.23 & 0.19 \\
\hline $\mathbf{3 k}$ & 0.19 & 0.17 \\
\hline $\mathbf{3 l}$ & 0.18 & 0.18 \\
\hline $\mathbf{3 m}$ & 0.18 & 0.18 \\
\hline $\mathbf{3 n}$ & 0.22 & 0.26 \\
\hline
\end{tabular}

